Exploring the molecular hub in plant elongation responses: regulation of PHYs and PIFs 


\section{Thesis committee}

\section{Promotor}

Prof. Dr C.S. Testerink

Professor of Plant Physiology

Wageningen University \& Research

\section{Co-promotor}

Dr S. van der Krol

Associate professor, Laboratory of Plant Physiology

Wageningen University \& Research

\section{Other members}

Prof. Dr L.F.M. Marcelis, Wageningen University \& Research

Prof. Dr K.H.W.J. ten Tusscher, Utrecht University

Prof. Dr R. Offringa, Leiden University

Prof. Dr H.J. Bosch, Wageningen University \& Research

This research was conducted under the auspices of the Graduate School of Experimental Plant Sciences. 


\title{
Exploring the molecular hub in plant elongation responses: regulation of PHYs and PIFs
}

\author{
Umidjon Shapulatov
}

\section{Thesis}

submitted in fulfilment of the requirements for the degree of doctor at Wageningen University

by the authority of the Rector Magnificus,

Prof. Dr A.P.J. Mol, in the presence of the

Thesis Committee appointed by the Academic Board to be defended in public on Tuesday 2 April 2019 at 4 p.m. in the Aula. 
Umidjon Shapulatov

Exploring the molecular hub in plant elongation responses: regulation of PHYs and PIFs,

194 pages.

PhD thesis, Wageningen University, Wageningen, the Netherlands (2019)

With references, with summary in English

ISBN: 978-94-6343-435-5

DOI: $10.18174 / 471540$ 


\section{CONTENTS}

Chapter 1

Chapter 2

Chapter 3

Chapter 4

Chapter 5

Chapter 6

General Discussion by Phytochrome Luciferase reporters

Feedback at the PIF4 locus: PIF4 is a negative regulator

65

of its own expression

A central role for MED25 in thermomorphogenesis

Functional intron-derived miRNAs and host-gene

expression in plants

Summary

Acknowledgements

Curriculum Vitae

Education Statement 



\section{Chapter-1}

General Introduction 


\section{Research for efficient horticulture.}

In horticulture compactness is a quality trait for ornamental plants. Compactness can be obtained by using chemical inhibitors of production (endogenous biosynthesis) of the phytohormone gibberellin (GA), which promotes plant elongation responses. However, the use of such chemicals in greenhouses is environmentally unfriendly and is being phased out. Therefore, alternative, more sustainable treatments are needed to keep plants compact. One option is the use of alternative light/temperature regimes. While the normal day regime in greenhouses is called +DIF (warm day, cold night), an alternative -DIF regime consists of an inversion of day and night temperature: the temperature during the day is kept cool, while the night temperature is kept high. Such -DIF treatment for many plant species results in a more compact stature, as -DIF inhibits elongation responses in plants (Patil and Moe, 2009; Bours et al., 2015). The research described here is part of the STW project 'compact plants'. The aim of the 'compact plant' project is to get a fundamental understanding of the molecular mechanisms that underly the elongation inhibition under -DIF and regulation of plant growth in general. The ultimate aim of the 'compact plant' STW project is to find suitable new protocols for horticulture to enhance the effect of -DIF or to find alternatives to -DIF treatment for the growth control of plants in greenhouses. The -DIF treatment can only be applied during a certain period of the year (cooling during the day is too expensive in summer). Therefore, enhancing the effectiveness of -DIF may also result in a longer period of the year during which -DIF can be applied.

\section{Insight into molecular control of plant elongation under-DIF at the start of this project.}

Previous research by Bours (Bours, 2014) has shown that different light/temperature combinations affect the overall capacity of a signal transduction pathway towards elongation. This signal transduction pathway is activated by perception of the key transcription factors Phytochrome Interacting Factor 4 (PIF4) and PIF5 (Wang et al., 2017). As the name suggests, these transcription factors interact with phytochromes (PHYs), of which the interaction with PHYB has been best described. When light activates PHYB the active Pfr form of PHYB is translocated to the nucleus where it binds to PIF4, leading to phosphorylation of PIF4 and eventually to targeting this protein for degradation (Bauer et al., 2004; Khanna et al., 2004; Park et al., 2004; Al-Sady et al., 2006; Oh et al., 2006; Shen et al., 2007; Shen et al., 2008). PIF4 
and PIF5 are central transcription factors for plant elongation responses, their activity is limited in the light through destabilisation by the interaction with activated PHYB, resulting in limited growth in the light. The transcription factors PIF4/5 target genes for auxin biosynthesis like YUCCA8, resulting in increased auxin levels (Bours et al., 2015). Auxin signaling subsequently activates genes for ACC synthases, leading to increased ethylene production and increased ethylene signalling. The ethylene signalling through EIN3 subsequently activates PIF3, which targets genes directly involved in cell wall loosening and cell expansion (Bours et al., 2015). The PIF3 protein stability is not only decreased through the interaction with PHYB but also through the interaction with light activated PHYA (Park et al., 2004; Ni et al., 2013). Thus, for elongation responses, PIFs are important and both PHYB and PHYA are of relevance. The research of Bours (Bours, 2014)showed that PIF4/5 act upstream in this signal transduction pathway because the elongation defect in mutants lacking PIF4 and PIF5 can be complemented by auxin or by ethylene. In contrast, elongation effects in mutants lacking PIF3 are not complemented by auxin or ethylene(Bours et al., 2015). The model of the interactions involved in elongation responses as known at the end of the project of Bours (Bours, 2014) and at on-set of this project is presented in Figure 1.

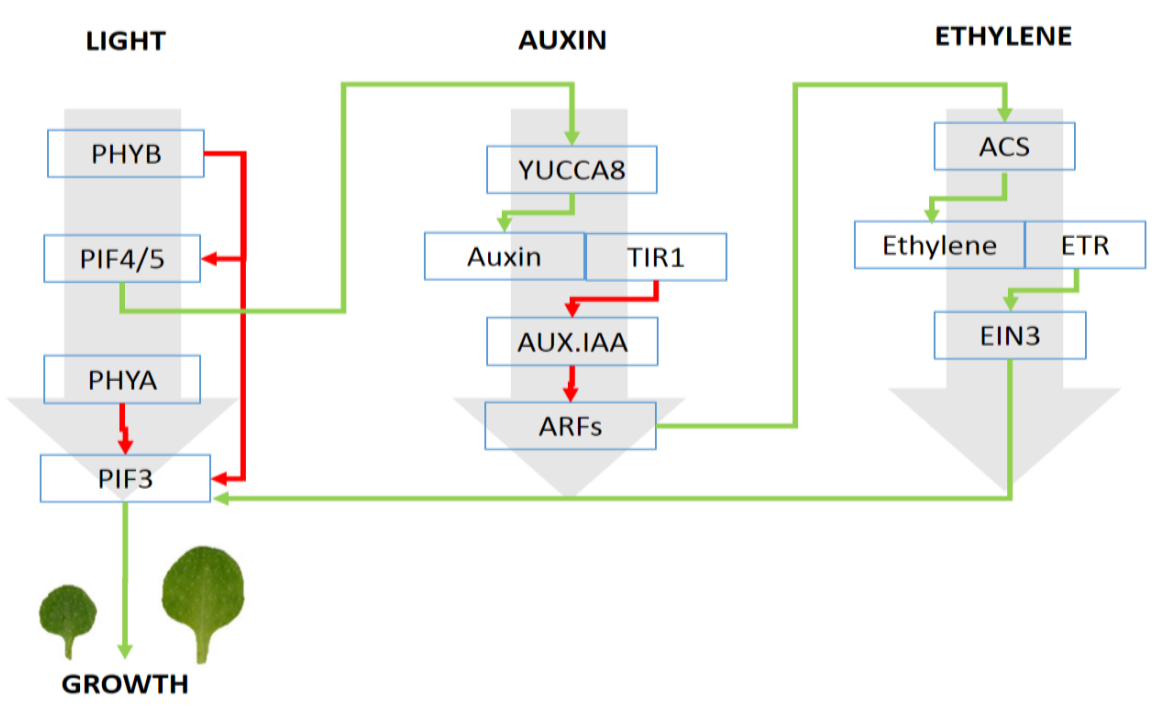

Figure 1. Signal transduction pathway towards elongation as elucidated at the start of this project

(Bours et al., 2015). Red arrows: negative interaction; Green arrows: positive interaction; Grey arrows: different signal transduction pathways. 


\section{The circadian clock is affected by -DIF.}

The signal transduction pathway towards elongation responses in plants as shown in Figure 1 is not static. Multiple components of the signal transduction pathway shown in Figure 1 are under control of the circadian clock (PIF4/5, YUCCA8, PHYB, PHYA). Moreover, the research of Bours (Bours, 2014) has shown that the -DIF treatment is also affecting the functioning of the clock itself. Initially, this was determined by measuring clock controlled leaf movement. However, the activity of clock genes was also monitored directly, using reporter plants expressing different firefly luciferase clock reporters (ffLUC reporters). The measurements on clock regulated leaf movement and on clock genes themselves showed that the different DIF conditions have a direct effect on phase and amplitude of clock genes and clock controlled processes. The phase shift for the different clock genes under -DIF is not the same: some clock genes show an earlier phase, while other clock genes show a later phase under-DIF (Bours et al., 2015). This means that the coordination of the different clock controlled processes will also not be the same under -DIF. Since all of the components in the signal transduction pathway in Figure 1 show some form of regulation by the circadian clock, the altered phases for each of the components will lead to some mismatches in peak activities over time, resulting in a bottleneck in the signal transduction chain. Thus -DIF alters the overall signalling capacity at different times of the day. This is most prominent for auxin and ethylene signalling under -DIF. Indeed, the inhibitory effect on plant elongation of -DIF can be complemented by adding either auxin or ethylene (Bours et al., 2015). The auxin signalling is especially limited during the day, suggested a lower than normal input by PIF4/5 in this signal transduction cascade during the day. The lower activity of PIF4/5 during the day under -DIF could be due to the interaction with light activated PHYB during the day. This was confirmed by Bours by demonstrating that a phyB mutant showed a reduced sensitivity to the suppression of elongation under -DIF (Bours et al., 2013).

\section{Interactions between Phytochromes: from cotton to Arabidopsis.}

Because of the strong and direct effect of light activated phytochromes on PIFs I was interested to determine whether transcriptional input of PHY gene expression can be a limiting factor for the activity of PIFs. The interest in studying the interactions between different phytochromes comes from my background in Uzbekistan where cotton is a major 
crop. In 2014, Abdurakhmonov et al had shown that inhibition of the cotton PHYA gene by an RNAi construct resulted in several improvements of the cotton plant (Abdurakhmonov et al., 2014). The cotton plants in which PHYA expression was decreased through post transcriptional gene silencing showed more vigorous root- and vegetative-growth, exhibited early-flowering and a significantly improved length of the cotton fiber (Abdurakhmonov et al., 2014). Analysis of these cotton plants with silenced PHYA indicated that the silencing of the cotton PHYA gene resulted in overexpression of the endogenous cotton PHYB gene, suggesting that the cotton PHYA normally suppresses the activity of the cotton PHYB gene. Indeed, similar improvements in cotton had been obtained before by overexpression of the Arabidopsis PHYB gene in cotton (Rao et al., 2011). It was not investigated whether the overexpression of the Arabidopsis PHYB in cotton resulted in suppression of cotton PHYA expression. Overall, this raises the question what is causal for the cotton improvement: down-regulation of cotton PHYA or up-regulation of the cotton PHYB? These experiments also reveal that there can be substantial interaction between different phytochromes and raises the question whether the decreased sensitivity of Arabidopsis phyB mutants is actually coming from increased expression of other PHY genes. Based on the observations in cotton we wondered how PHYs interact in Arabidopsis and how this contributes to the control of plant growth responses as function of light and temperature. While some interactions between phytochromes have been studied in Arabidopsis at the genetic level, for instance, by scoring hypocotyl elongation under Red (R) or Far Red (FR) light in single and double phytochrome mutants, these genetic interactions have not been directly linked to changes in the transcriptional regulation of the different PHY genes.

\section{Phytochrome signaling capacity a function of other PHYs?}

Phytochromes are photoreceptors that have important role in elongation responses. In Arabidopsis phytochromes are encoded by five genes (PHYA-PHYE) (Lin, 2002). All phytochrome proteins assemble into active photoreceptors by addition of a chromophore. The fully functional phytochrome proteins are activated by $R$ light and inactivated by FR light. Among the members of the PHY gene family, PHYA and PHYB have the most prominent function. PHYA is abundant in seeds and dark-grown seedlings and plays a crucial role during first light responses. Moreover, PHYA is the only phytochrome that also responds to FR light (Tepperman et al., 2006). In contrast to the other (stable) phytochrome proteins, activated PHYA is rapidly degraded in light. PHYB is the dominant phytochrome for regulating growth 
responses in continuous light-grown plants (Reed et al., 1998). PhyC, D and E have multiple functions throughout plant development and act redundant with PHYB responses (Aukerman et al., 1997; Franklin et al., 2003; Monte et al., 2003). Although the interaction between phytochromes has been studied at the genetic level, it is not known how individual PHYs affect the expression of other PHY genes.

Previous studies demonstrated that the length of seedling hypocotyl or the length of leaf petiole and leaf movement are significantly altered under-DIF condition compared to +DIF (Bours et al., 2015). In this elongation cascade Phytochrome B regulates both upstream (PIF4/5) and downstream (PIF3) the stability of PIF protein, while PHYA may also regulate PIF3 protein levels (Fig.1). Under -DIF auxin becomes limiting for elongation (Bours et al., 2015), suggesting that PIF4/5 activity is limiting under -DIF. This limited PIF4/5 activity may either be caused by lower expression of PIF4/5 genes under -DIF or by higher activity of PHYB targeting PIF4/5 protein for destruction. In this research we therefore aimed at an inventory of the PHY expression profiles and PIF expression profiles under different light/temperature conditions to determine whether the coincidence in PHY and PIF gene activity contributes to overall PIF activity for elongation. This research makes use of firefly luciferase (LUC) reporter plants expressing pPHY:LUC or pPIF:LUC expression constructs. For each of the phytochrome genes a PHY:LUC reporter was made and transformed to Arabidopsis WT (Col-0). Selected homozygous lines where then crossed into the different phytochrome single mutants, resulting in a set of 30 reporter plants with which we have analysed the expression or and interaction between the different PHY genes. Analysis of these reporter plants was done using LUMINATOR, a sensitive camera system to image LUC activity in plants, with LED light and temperature control.

\section{New components for the growth model: MED25 and BZR1.}

This research addresses the control of plant growth with a focus of the role of phytochromes. However, also other mutants that show altered phytochrome signaling and elongation are of potential interest, as they help to understand all the steps involved in elongation responses. During this research two factors were added to the conceptual model: BZR1 and MED25. BZR1 is a transcription factor that is activated upon brassinosteroid (BR) signaling and is required for the elongation response mediated by the action of PIFs (Ibañez 
et al., 2018; Martínez et al., 2018). Full knock-out mutants of BZR1 are embryo lethal, but in research a gain-of-function mutant bzr1-1D has been used. The gain of function mutation in bzr1-1D leads to a constitutive activation and stabilization of the BZR1 protein and can therefore be considered as a BZR1 overexpression line, in which BZR1 activity is not dependent on activation by endogenous BR signaling (Wang et al., 2002). An interesting feature of the bzr1-1D mutant is that it hardly has a hypocotyl growth phenotype when plants are grown at normal temperature $\left(22^{\circ} \mathrm{C}\right)$, but bzr1-1D does have an exaggerated hypocotyl elongation response at higher $\left(27^{\circ} \mathrm{C}\right)$ temperature (Ibañez et al., 2018). The floral organs of bzr1-1D are enlarged compared to WT Arabidopsis plant flowers.

Another mutant identified from literature is the pft1-2 mutant. PFT1 encodes a nuclear protein that acts in the PHYB signaling pathway. Mutations in PFT1 alter flowering responses under suboptimal light conditions. For instance, mutants in PFT1 show reduced responses to far red (FR) light. However, PFT1 action is pleotropic as it integrates environmental factors such as light quality (Klose et al., 2012), JA dependent defenses (Kidd et al., 2009; Zhu et al., 2014) and auxin signaling (Raya-Gonzalez et al., 2014; Ito et al., 2016). PFT1 encodes subunit 25 of the conserved Mediator protein complex and is therefore also called MED25. The mediator complex consists of up to 30 proteins, and this complex of proteins functions as adaptor between a specific sub-set of transcription factors and the general transcription machinery containing the RNA polymerase II (Elfving et al., 2011; Kazan, 2017). In our research we found that MED25 is involved in the transcriptional activity of PIF4 and BZR1.

\section{Transcript and miRNA profiling under -DIF.}

The research on genes functioning in the elongation responses of Arabidopsis using selected LUC-reporter plants was complemented by a broader profiling of transcripts to study the more general effect of -DIF treatment. Profiling was done at two key time points during plant growth: at the end of night and at the end of day, both for plants grown under +DIF and under -DIF. In order to study the role of PHYB in the differential gene expression under +DIF/DIF, the same expression profiling was also done at the same time points for the phyB-9 mutant. In addition to mRNA profiling we also included profiling of miRNAs at these two key time points to determine whether some of the responses under -DIF can be attributed to altered miRNA activity. MicroRNAs are short 21-24 nucleotide length of non-coding small 
RNAs that function in RNA silencing, either by destabilization of target mRNA or by blocking target mRNA translation. It had already been established that miRNAs play a central role in the interaction between phytohormones in plants (Curaba et al., 2014). The reason to investigate whether there is a role for miRNAs in the response to -DIF is that auxin is a key factor in -DIF responses. The auxin responses are mediated by Auxin Response Factor (ARF) transcription factors and for multiple ARFs it has been shown that they are regulated at the post transcriptional level by miRNAs. For instance, ARF16 and ARF17 are targeted by miRNA160 and ARF6, ARF8 as well ARF19 are targeted by miRNA167 (Mallory et al., 2005). In addition, PHYB has been implemented in the control of miRNA biosynthesis (Sun et al., 2015; Sun et al., 2018). Because PHYB plays a prominent role in the -DIF response the question was whether part of the -DIF responses is by miRNAs affecting auxin signaling in plants. While the miRNA sequencing results are not presented in this thesis, as they need further bioinformatics analysis to be finalized, some intriguing preliminary observations from this research are presented and discussed in the final discussion chapter 6.

\section{Engineering for potential applications in plants.}

The insights into the molecular control of growth in plants has its application in new protocols for greenhouses to keep plants compact. However, the same insights may also be applied in cases where it may be desirable to obtain larger plants or larger flowers. For instance, the mutant bzr1-1D and pft1-2 both have enlarged flowers and during this research we found that the bzr1-1D/pft1-2 double mutant has even larger flowers. This may have potential applications in ornamentals or crops for which the flower is harvested. For instance, if the same effect can be obtained in cotton by introducing a bzr1-1D overexpression construct and a RNAi construct targeting the MED25 of cotton, this could potentially result in bigger cotton flowers. Cotton produces fibers that grow in the protective case (boll) around the seeds of the cotton plants. The question is whether larger cotton flowers can host more cotton seeds that produce the cotton fibers or will allow for more space for the cotton fibers to grow. However, cotton is not an easy plant to transform and introduction of two different construct would require quit some effort. In general, many plant manipulations may benefit from overexpression of one gene, while at the same time silencing another gene. For instance, in metabolic engineering a specific terpene synthase may be used for overexpression (Wang et 
al., 2016) while an RNAi construct may target a side branch in the terpene biosynthesis pathway to direct all synthesis to the desired product. We therefore developed a novel strategy to obtain such dual manipulations in a single gene construct. The concept of this novel strategy comes from the observation that some natural miRNAs are located in the intron of a host gene in plants. Arabidopsis has 37 intron-derived miRNAs and rice has 181 intron-derived miRNAs (Yang et al., 2012). Although in plants it had not been established that the production of such intron-derived miRNAs is without interference of the host gene expression we used this concept from nature to design an artificial transgene for overexpression with an intron containing a miRNA sequence targeting another gene of interest. Using a LUC reporter this concept was proven to function in plants (Chapter $\mathbf{5}$ ).

\section{Outline of this thesis.}

In chapter 2, We researched the question how phytochrome genes affect each other at the transcriptional level. This was studied using a set of PHY-LUC reporter plants for each of the five phytochrome genes of Arabidopsis. The PHY-LUC reporter activity was studied in WT plants and in the five single PHY KO-mutants. Results showed that in seedlings phytochrome genes do affect each other at the transcript level, but in more mature stages of development (e.g. rosette plants) not many of these interactions remain. These studies also resulted in several novel discoveries. For instance, (1) we found that PHYD is a constitutive repressor of PHYA gene activity, (2) we found that PHYB and PHYA are upregulated under FR light, but in a different way, (3) we found that the upregulation of $P H Y B$ under FR is dependent on PHYB, PHYE and PIF4 (4) we found that the upregulation of PHYA under FR is dependent on PHYE.

In chapter 3 We studied part of the complex feedback regulation at the PIF4 locus. During this research period it had become known that BRR1 is a key regulator of PIF4 expression, especially under higher temperature. Moreover, activation of PIF4 by BZR1 results in an indirect feedback loop through BR synthesis, BR signaling and further activation of BZR1. It was thought that this positive feedback regulation of PIF4 is kept under control by factors acting at the post-transcriptional level on the PIF4 protein. However, we demonstrate in 
chapter 3 that PIF4 is a negative regulator of its own expression and acts dominant over the positive action of BZR1 on PIF4 expression. In addition, we show that PIF4 regulates PHYB expression, adding another negative feedback loop to the control of PIF4 activity.

In chapter 4, We studied the effect of MED25 on the activity of BZR1 and PIF4. Using a split luciferase assay we show that MED25 can bind to the PIF4 and BZR1 protein in planta. Moreover, we also show that MED25 can interact with the histone modifying deacetylase enzyme HDA9. Mapping of the MED25 interacting domain shows that both PIF4 and HDA9 bind to the poly-Q domain of MED25. Recently a role of HDA9 in warmth induced elongation responses was investigated by Martijn van Zanten at Utrecht University (manuscript submitted). Although the effect of HDA9 was clearly demonstrated, it was not clear how HDA9 is recruited to promoters with bound BZR1 or PIF4. The dual binding capacity of MED25, interacting both with the transcription factors bound to promoter sites and the interacting with HDA9, may explain how HDA9 is recruited to promoters. We demonstrate that MED25 affects the transcriptional activity of PIF4, but that PIF4 expression in the MED25 mutant pft12 is uncoupled from target gene expression and elongation responses.

In chapter 5, We describe how multiple manipulations of growth of plants may be reached in principle through a single expression construct. By placing a miRNA sequence (named artificial intron miRNA: aimiR) into the intron of a luciferase genomic gene (gLUC) I could test whether this results in simultaneous host transgene (LUC) expression and miRNA production. After adjusting the insertion cloning strategy, the ffgLUC aimiR-319a gene showed dual functionality with correct splicing of ffgLUC and efficient silencing of TEOSINTE BRANCHED1/CYCLOIDEA/PROLIFERATING CELL FACTOR1 transcription factor genes targeted in-trans by aimiR-319a. The same principle was also demonstrated with an aimiR-LUC which targets the transgene ffLUC in-cis. Silencing of endogenous target genes by aimiRNA or amiRNA is efficient both in transient assays and stable transformants. This concept therefore adds new options to engineering of plant traits that require multiple gene manipulations.

In the last chapter (chapter 6) I discuss some of the difficulties we encountered and many questions that remain after the different discoveries that were made during this research. I discuss experiments that are needed to answer some of the remaining questions. I discuss the seeming discrepancies between our own experimental results with PIF4 
overexpression and those found in literature. In this chapter I also speculate on the role of MED25 in the action of BZR1 and PIF4 and I present an update of Figure 1 in a new extended conceptual model of the signal transduction towards elongation. During this research we also discussed that PIF4 proteins with a tag do not behave the same as endogenous PIF4 without tag, which explained discrepancies between our results and literature. 


\section{REFERENCE}

Abdurakhmonov IY, Buriev ZT, Saha S, Jenkins JN, Abdukarimov A, Pepper AE (2014) Phytochrome RNAi enhances major fibre quality and agronomic traits of the cotton Gossypium hirsutum L. Nat Commun 5: 3062

Al-Sady B, Ni W, Kircher S, Schafer E, Quail PH (2006) Photoactivated phytochrome induces rapid PIF3 phosphorylation prior to proteasome-mediated degradation. Mol Cell 23: 439-446

Aukerman MJ, Hirschfeld M, Wester L, Weaver M, Clack T, Amasino RM, Sharrock RA (1997) A deletion in the PHYD gene of the Arabidopsis Wassilewskija ecotype defines a role for phytochrome $D$ in red/far-red light sensing. Plant Cell 9: 1317-1326

Bauer D, Viczian A, Kircher S, Nobis T, Nitschke R, Kunkel T, Panigrahi KC, Adam E, Fejes E, Schafer E, Nagy F (2004) Constitutive photomorphogenesis 1 and multiple photoreceptors control degradation of phytochrome interacting factor 3, a transcription factor required for light signaling in Arabidopsis. Plant Cell 16: 1433-1445

Bours R (2014) Antiphase light and temperature cycles disrupt rhythmic plant growth : the Arabidopsis jetlag. Proefschrift Wageningen University ter verkrijging van de graad van doctor in het jaar 2014

Met literatuuropgave. - Met samenvatting in het Engels en Nederlands. Wageningen University, Wageningen

Bours R, Kohlen W, Bouwmeester HJ, van der Krol A (2015) Thermoperiodic control of hypocotyl elongation depends on auxin-induced ethylene signaling that controls downstream PHYTOCHROME INTERACTING FACTOR3 activity. Plant Physiol 167: 517530

Bours R, van Zanten M, Pierik R, Bouwmeester H, van der Krol A (2013) Antiphase light and temperature cycles affect PHYTOCHROME B-controlled ethylene sensitivity and biosynthesis, limiting leaf movement and growth of Arabidopsis. Plant Physiol 163: 882-895

Curaba J, Singh MB, Bhalla PL (2014) miRNAs in the crosstalk between phytohormone signalling pathways. J Exp Bot 65: 1425-1438

Elfving N, Davoine C, Benlloch R, Blomberg J, Brannstrom K, Muller D, Nilsson A, Ulfstedt M, Ronne H, Wingsle G, Nilsson O, Bjorklund S (2011) The Arabidopsis thaliana Med25 mediator subunit integrates environmental cues to control plant development. Proc Natl Acad Sci U S A 108: 8245-8250

Franklin KA, Praekelt U, Stoddart WM, Billingham OE, Halliday KJ, Whitelam GC (2003) Phytochromes $B, D$, and $E$ act redundantly to control multiple physiological responses in Arabidopsis. Plant Physiol 131: 1340-1346

Ibañez C, Delker C, Martinez C, Bürstenbinder K, Janitza P, Lippmann R, Ludwig W, Sun H, James GV, Klecker M, Grossjohann A, Schneeberger K, Prat S, Quint M (2018) Brassinosteroids Dominate Hormonal Regulation of Plant Thermomorphogenesis via BZR1. Current Biology 28: 303-310.e303

Ito J, Fukaki H, Onoda M, Li L, Li C, Tasaka M, Furutani M (2016) Auxin-dependent compositional change in Mediator in ARF7- and ARF19-mediated transcription. Proc Natl Acad Sci U S A 113: 6562-6567

Kazan K (2017) The Multitalented MEDIATOR25. Front Plant Sci 8: 999 
Khanna R, Huq E, Kikis EA, Al-Sady B, Lanzatella C, Quail PH (2004) A novel molecular recognition motif necessary for targeting photoactivated phytochrome signaling to specific basic helix-loop-helix transcription factors. Plant Cell 16: 3033-3044

Kidd BN, Edgar Cl, Kumar KK, Aitken EA, Schenk PM, Manners JM, Kazan K (2009) The mediator complex subunit PFT1 is a key regulator of jasmonate-dependent defense in Arabidopsis. Plant Cell 21: 2237-2252

Klose C, Buche C, Fernandez AP, Schafer E, Zwick E, Kretsch T (2012) The mediator complex subunit PFT1 interferes with COP1 and HY5 in the regulation of Arabidopsis light signaling. Plant Physiol 160: 289-307

Lin C (2002) Blue light receptors and signal transduction. Plant Cell 14 Suppl: S207-225

Mallory AC, Bartel DP, Bartel B (2005) MicroRNA-directed regulation of Arabidopsis AUXIN RESPONSE FACTOR17 is essential for proper development and modulates expression of early auxin response genes. Plant Cell 17: 1360-1375

Martínez C, Espinosa-Ruíz A, de Lucas M, Bernardo-García S, Franco-Zorrilla JM, Prat S (2018) PIF4-induced BR synthesis is critical to diurnal and thermomorphogenic growth. The EMBO Journal

Monte E, Alonso JM, Ecker JR, Zhang Y, Li X, Young J, Austin-Phillips S, Quail PH (2003) Isolation and characterization of phyC mutants in Arabidopsis reveals complex crosstalk between phytochrome signaling pathways. Plant Cell 15: 1962-1980

Ni W, Xu SL, Chalkley RJ, Pham TN, Guan S, Maltby DA, Burlingame AL, Wang ZY, Quail PH (2013) Multisite light-induced phosphorylation of the transcription factor PIF3 is necessary for both its rapid degradation and concomitant negative feedback modulation of photoreceptor phyB levels in Arabidopsis. Plant Cell 25: 2679-2698

Oh E, Yamaguchi S, Kamiya Y, Bae G, Chung WI, Choi G (2006) Light activates the degradation of PIL5 protein to promote seed germination through gibberellin in Arabidopsis. Plant J 47: 124-139

Park E, Kim J, Lee Y, Shin J, Oh E, Chung WI, Liu JR, Choi G (2004) Degradation of phytochrome interacting factor 3 in phytochrome-mediated light signaling. Plant Cell Physiol 45: 968975

Patil GG, Moe R (2009) Involvement of phytochrome B in DIF mediated growth in cucumber. Scientia Horticulturae 122: 164-170

Rao AQ, Irfan M, Saleem Z, Nasir IA, Riazuddin S, Husnain T (2011) Overexpression of the phytochrome $B$ gene from Arabidopsis thaliana increases plant growth and yield of cotton (Gossypium hirsutum). J Zhejiang Univ Sci B 12: 326-334

Raya-Gonzalez J, Ortiz-Castro R, Ruiz-Herrera LF, Kazan K, Lopez-Bucio J (2014) PHYTOCHROME AND FLOWERING TIME1/MEDIATOR25 Regulates Lateral Root Formation via Auxin Signaling in Arabidopsis. Plant Physiol 165: 880-894

Reed JW, Elumalai RP, Chory J (1998) Suppressors of an Arabidopsis thaliana phyB mutation identify genes that control light signaling and hypocotyl elongation. Genetics 148: 1295-1310

Shen H, Zhu L, Castillon A, Majee M, Downie B, Huq E (2008) Light-induced phosphorylation and degradation of the negative regulator PHYTOCHROME-INTERACTING FACTOR1 from Arabidopsis depend upon its direct physical interactions with photoactivated phytochromes. Plant Cell 20: 1586-1602 
Shen Y, Khanna R, Carle CM, Quail PH (2007) Phytochrome induces rapid PIF5 phosphorylation and degradation in response to red-light activation. Plant Physiol 145: 1043-1051

Sun W, Xu XH, Wu X, Wang Y, Lu X, Sun H, Xie X (2015) Genome-wide identification of microRNAs and their targets in wild type and phyB mutant provides a key link between microRNAs and the phyB-mediated light signaling pathway in rice. Front Plant Sci 6: 372

Sun Z, Li M, Zhou Y, Guo T, Liu Y, Zhang H, Fang Y (2018) Coordinated regulation of Arabidopsis microRNA biogenesis and red light signaling through Dicer-like 1 and phytochromeinteracting factor 4. PLOS Genetics 14: e1007247

Tepperman JM, Hwang YS, Quail PH (2006) phyA dominates in transduction of red-light signals to rapidly responding genes at the initiation of Arabidopsis seedling deetiolation. Plant J 48: 728-742

Wang B, Kashkooli AB, Sallets A, Ting H-M, de Ruijter NCA, Olofsson L, Brodelius P, Pottier M, Boutry M, Bouwmeester $H$, van der Krol AR (2016) Transient production of artemisinin in Nicotiana benthamiana is boosted by a specific lipid transfer protein from A. annua. Metabolic Engineering 38: 159-169

Wang L, Wu LM, Greaves IK, Zhu A, Dennis ES, Peacock WJ (2017) PIF4-controlled auxin pathway contributes to hybrid vigor in \&lt;em\&gt;Arabidopsis thaliana\&lt;/em\&gt. Proceedings of the National Academy of Sciences 114: E3555

Wang Z-Y, Nakano T, Gendron J, He J, Chen M, Vafeados D, Yang Y, Fujioka S, Yoshida S, Asami T, Chory J (2002) Nuclear-Localized BZR1 Mediates Brassinosteroid-Induced Growth and Feedback Suppression of Brassinosteroid Biosynthesis. Developmental Cell 2: 505-513

Yang GD, Yan K, Wu BJ, Wang YH, Gao YX, Zheng CC (2012) Genomewide analysis of intronic microRNAs in rice and Arabidopsis. J Genet 91: 313-324

Zhu Y, Schluttenhoffer CM, Wang P, Fu F, Thimmapuram J, Zhu JK, Lee SY, Yun DJ, Mengiste T (2014) CYCLIN-DEPENDENT KINASE8 differentially regulates plant immunity to fungal pathogens through kinase-dependent and -independent functions in Arabidopsis. Plant Cell 26: 4149-4170 


\title{
CHAPTER-2
}

Novel interactions under Red, Far-Red, and Blue light by Phytochrome Luciferase reporters

\author{
Umidjon Shapulatov \\ Mark van Hoogdalem \\ Mara Meisenburg \\ Alexander van der Hall \\ Wim van leperen \\ Xiu-Ping Gao \\ Maarten van Wassenaar \\ Christa Testerink \\ Alexander van der Krol
}

In preparation for publication 


\begin{abstract}
For Red (R) and Far Red (FR) light perception, Arabidopsis has five phytochrome (PHY) genes, of which only PHYA has an established role in responses to FR. Here we investigated the transcriptional activity of the five pPHY:LUC reporters as function of development, as function of individual single phytochrome mutations and as function of R, FR or Blue light conditions. These studies reveal that PHYD is a constitutive repressor of PHYA-reporter activity and that PHYB and PHYA reporter activity is strongly up-regulated under FR, while this response to FR is not affected by the classical FR sensor PHYA but by PHYB and PHYE. Moreover, we show that the Phytochrome Interacting Factor PIF4 is in part responsible for the regulation of the PHYB reporter under FR but not for the expression of PHYD reporter. The responses of the endogenous PHYA and PHYB genes and suppression of PHYA by PHYD under FR were smaller as observed for the LUC reporters. These studies reveal novel interactions between phytochromes and reveal novel sensors for FR responses in plants. Such insights may provide a new fundamental basis for manipulating plant growth using LED lights in indoor farming.
\end{abstract}

Key words: PHYTOCHROME, LED, Luciferase reporter plants, transcription 


\section{Introduction}

Currently, about $80 \%$ of the total global arable land area is designated to soil-based farming. However, more intense farming efforts are needed, especially in urban areas, to meet the growing global food demand in the near future. One way in which farming may be intensified in an urban setting is through the use of indoor (vertical) farming, using LED lights for plant growth. However, LED lights are different from sunlight in their spectral properties, and some of our fundamental insights into photobiology of plants may need revisiting for artificial LED-light conditions in order to make optimal use of LED lights in agriculture.

Plant growth and development in the dark (skotomorphogenesis) is fundamentally different from growth and development in the light (photomorphogenesis). The most important photoreceptors that control plant growth as function of the Red (R) and Far-Red (FR) light spectrum are a family of phytochrome (PHY) genes, which in Arabidopsis consist of PHYA-PHYE (Bae and Choi, 2008). Phytochromes are produced in the inactive red (R) light absorbing Pr form and upon perception of red light, the inactive far red (FR) light absorbing Pr form changes to the active Pfr state to trigger both responses in the cytosol (Paik et al., 2012) and in the nucleus (Nagy and Schafer, 2002; Nagatani, 2004; Kevei et al., 2007; Van Buskirk et al., 2012; Klose et al., 2015). In the nucleus phytochrome protein interacts with multiple Phytochrome Interacting Factors (PIFs) to mediate light transcriptional responses (Huq et al., 2004; Castillon et al., 2007; Leivar and Quail, 2011). While phytochromes are activators, PIFs are considered repressors of photomorphogenesis, because phytochrome Pfr promotes the turnover of PIF proteins (Park et al., 2012; Xu et al., 2015). The interactions between phytochromes and PIFs do not only result in degradation of the PIFs, but also in co-degradation of the phytochrome protein (Monte et al., 2004; Khanna et al., 2007; Al-Sady et al., 2008; Leivar et al., 2008; Leivar and Quail, 2011; Ni et al., 2013). The function of Pfr in the nucleus is controlled by multiple nuclear factors that are involved in nuclear Pfr stability. It has been shown that PIFs regulate phyB-E protein stability through COP1/DET/FUS (Jang et al., 2010). In addition, PIFs and PHYs interact with a CUL3-based E3 ubiquitin ligases complex containing the Bric-a-Brac/Tramtrack/Broad Complex (BTB)-domain containing substrate adaptor LightResponse (LRB). Presumably PIFs and PHY are co-degraded by interaction between a CUL3LRB-PIF complex and a CUL3-LRB-PHY complex, through dimerization of the LRBs (Christians 
et al., 2012). In addition, nuclear Pfr shows a slow reversion to $\mathrm{Pr}$ in the dark and this darkreversion is accelerated under higher temperature. Thus, phytochrome Pfr levels in the nucleus function as temperature sensor (Jung et al., 2016; Legris et al., 2016). Translocation of PHY proteins into nucleus is required for the nuclear signaling and the translocation of PHYA Pfr protein into the nucleus is controlled by the FAR-RED ELONGATED HYPOCOTYL 1 (FHY1) AND FHY1-LIKE (FHL) (Genoud et al., 2008). PHYs also have a function in the cytosol where they control translation of specific mRNAs (Paik et al., 2012). The stability of the pool of cytosolic Pfr is regulated by cytosolic factors, explaining why the dynamics of nuclear PIF protein turnover and total PHY protein turnover may not be the same.

Our understanding of phytochrome action can not only come from studying downstream signaling of PHYs, but should also include an understanding of the transcriptional regulation of phytochrome gene themselves, as this ultimately determines the PHY protein input into the signaling cascades. Regulation of phytochrome gene transcription has not been studied extensively. It is known the phytochrome gene transcription is regulated by the circadian clock (Toth et al., 2001), while the clock is entrained through phytochrome signaling (Somers et al., 1998). This already implies a complex feedback regulation between phytochrome gene activity and the clock. Moreover, in seedlings, phytochromes influence each other's function (Sanchez-Lamas et al., 2016), indicating that PHY gene transcription is also function of R:FR light quality. For PHYA this light quality dependence of transcription was recently explained by the fact that PIF4 and PIF5 proteins target the PHYA promoter (Seaton et al., 2018), while PIFs stability is determined by light activated phytochrome Pfr (Lorrain et al., 2008; Foreman et al., 2011).

Activity of phytochromes is mostly studied at the protein level, through activation of Pf to Pfr as function of R:FR light conditions. Usually it is assumed that no dramatic effects occur at the level of PHY gene transcription by a given light treatment. However, the artificial spectral composition of LED lights may need verification of this assumption if we want to fully understand plant growth responses under LED lights. Indeed, there is no comprehensive and systematic analysis of PHY gene transcription as function of (LED) light quality. Therefore, we investigated PHY gene transcription as function of (LED) light quality, but also as function of development and as function of phytochrome signaling. Dynamic transcriptional responses in 
planta can be conveniently monitored using firefly luciferase reporter (Millar et al., 1992). In order to monitor phytochrome gene transcriptional activity we therefore developed a full set of five pPHY:LUC Arabidopsis Col-0 reporter lines (pPHYA:LUC, pPHYB:LUC, pPHYC:LUC, pPHYD:LUC and pPHYE:LUC). Each of the five pPHY:LUC reporter plants was crossed into each of the single phytochrome mutant backgrounds, resulting in a total of 30 reporter lines. Analysis of the LUC activity in these lines shows that in seedlings there are numerous interactions between phytochromes at the transcription level that change from seedling to mature rosette stage. The diurnal pPHY:LUC activity was monitored under a photoperiod of R, FR or B LED light. These measurements gave the unexpected result of strong upregulation of PHYB and PHYA gene activity during the pure FR photoperiod. Moreover, this induction by FR was not dependent on the classical FR light sensor PHYA. These studies also reveal PHYD as a constitutive suppressor of PHYA gene activity. Finally, PPHY:LUC activity was measured in blocks of three hours under different ratio of R:FR, mimicking different levels of shade. Expression analysis of the PPHY:LUC reporters in the different phytochrome mutant backgrounds revealed that the strong upregulation of PHYA under FR is a function of PHYE. These studies show an unexpected complex regulation of PHY reporter expression, uncovering a strong constitutive interaction between PHYA and PHYD under all light conditions, a conditional strong interaction between PHYA and PHYE under FR light and a strong and direct induction of PHYB reporter expression under FR, which is in part dependent on PHYB and PHYE. The validation of endogenous PHYA and PHYB expression under FR light shows qualitatively the same response but is qualitatively much reduced compared to the response of the PHYA and PHYB LUC reporters, raising the question which additional layer of endogenous PHYA and PHYB expression is not captured by the PPHY:LUC reporters. 


\section{RESULTS}

\section{Construction of thirty PPHY:LUC reporter lines}

In order to study the expression of the different phytochrome genes (PHYs) in WT and phytochrome mutant backgrounds, the upstream 2-2.5 kb promoter of each of the five Arabidopsis PHY genes was fused to the firefly luciferase (LUC) coding sequence in binary expression vectors (Toth et al., 2001). After transformation of the expression constructs to agrobacterium the different pPHY:LUC reporter constructs were introduced into Arabidopsis WT (Col-0) by agrobacterium mediated floral dip transformation (Zhang et al., 2006). For each of the PPHY:LUC reporters a minimum of ten primary transformants were screened for PPHY:LUC activity and one representative transformed plant was selected and developed into a homozygous reporter line expressing either PPHYA:LUC, PPHYB:LUC, PPHYC:LUC, pPHYD:LUC or PPHYE:LUC. Subsequently, each of the five homozygous PPHY:LUC reporter plants was crossed to each of five phy-mutant plants. For these crossings we used Salk T-DNA insertion lines for PHYA, C, D, E and a point mutation line for PHYB gene (phyB-9) (Fig.S1). From the T3 generation that was derived from these crosses the plants homozygous for the PPHY:LUC reporter and homozygous for the phytochrome mutation were selected. Phytochrome mutant backgrounds were selected based on seedling growth characteristics under specific light conditions and mutant background was confirmed by PCR analysis of genomic DNA using specific primers (Table S2) (Nagatani et al., 1993; Hennig et al., 1999; Balasubramanian et al., 2006; Chen et al., 2013). By crossing the PPHY:LUC reporter into the different phy-mutant backgrounds, the expression between WT and mutant lines with the same reporter can be compared directly as for both the WT and the phy mutant the PPHY:LUC reporter is inserted at the same chromosomal location. In this way a 'position effect' from independent transformation events is prevented. All pPHY:LUC reporter lines and constructs are listed in Table S1. Figure-1 shows representative images of the luciferase activity in all WT reporter lines for rosette plants at three weeks after germination. The image shows that the absolute level of PPHY:LUC expression is not the same for the different PHY promoters. At the rosette stage the PHYA and PHYC promoters show the strongest transcriptional activity, while the transcription from the PHYE promoter very weak. 

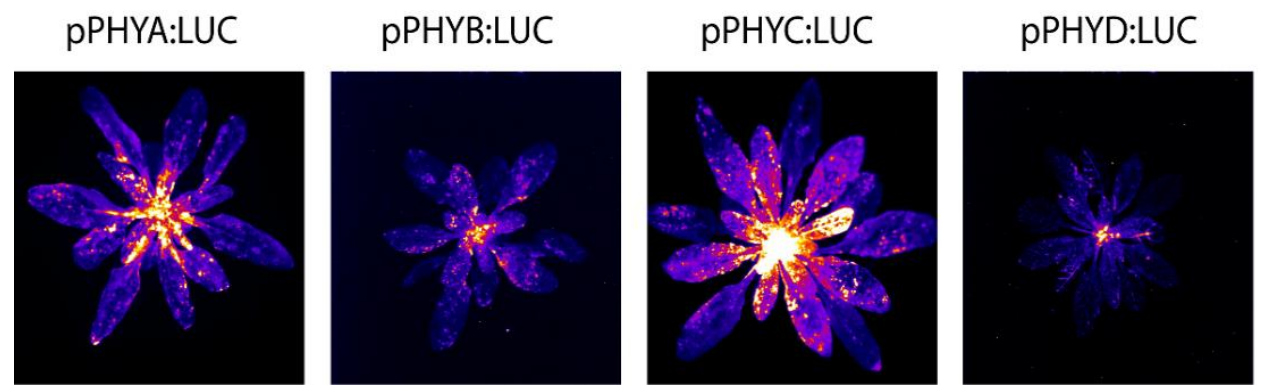

PPHYE:LUC

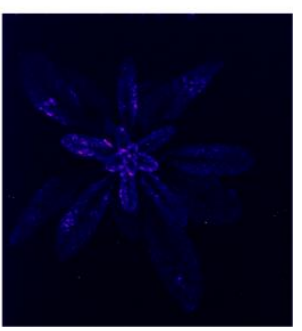

Figure-1. pPHY:LUC reporter plants. Relative luciferase activity was captured in 25-day old rosette plants sprayed with $1 \mathrm{mM}$ luciferin-D. LUC activity image capturing was by seven minutes exposure time. PPHYC:LUC shows the highest activity, while pPHYE:LUC is a little above background.

\section{PHYs interactions at transcription level in seedlings mostly absent in rosette plants}

The pPHY:LUC reporter plants were used to monitor PPHY:LUC activity at different stages of plant development, from 7 and 14 day old seedlings to 25 -day old plants. For this plants grown in growth cabinets under a 12L/12D diurnal light regime using fluorescent white lights (WL). At each of the three developmental stages, plants were pre-sprayed with the substrate luciferin one day in advance of the LUC activity imaging in order to deplete activity from previously accumulated luciferase protein. For all three developmental stages the LUC activity imaging was performed at $11 \mathrm{am}$, directly after transfer from the growth cabinet to LUMINATOR. This time point is at or close to the phase of all pPHY:LUC reporters as determined in seedlings (Toth et al., 2001). The average relative LUC activity was quantified for each of the reporter lines (Figure 2). The results indicate that in 7-day-old seedlings there is extensive interaction between the different PHYs, as indicated by altered pPHY:LUC expression in WT and phy mutant background. However, the genetic interactions between phytochromes at the transcription level is diminished in 14 day old seedlings (Figure 2). Most remarkable is the consistent elevated level of PHYA expression in the phyD mutant background, indicating that PHYD is a constitutive suppressor of PHYA gene transcription. 

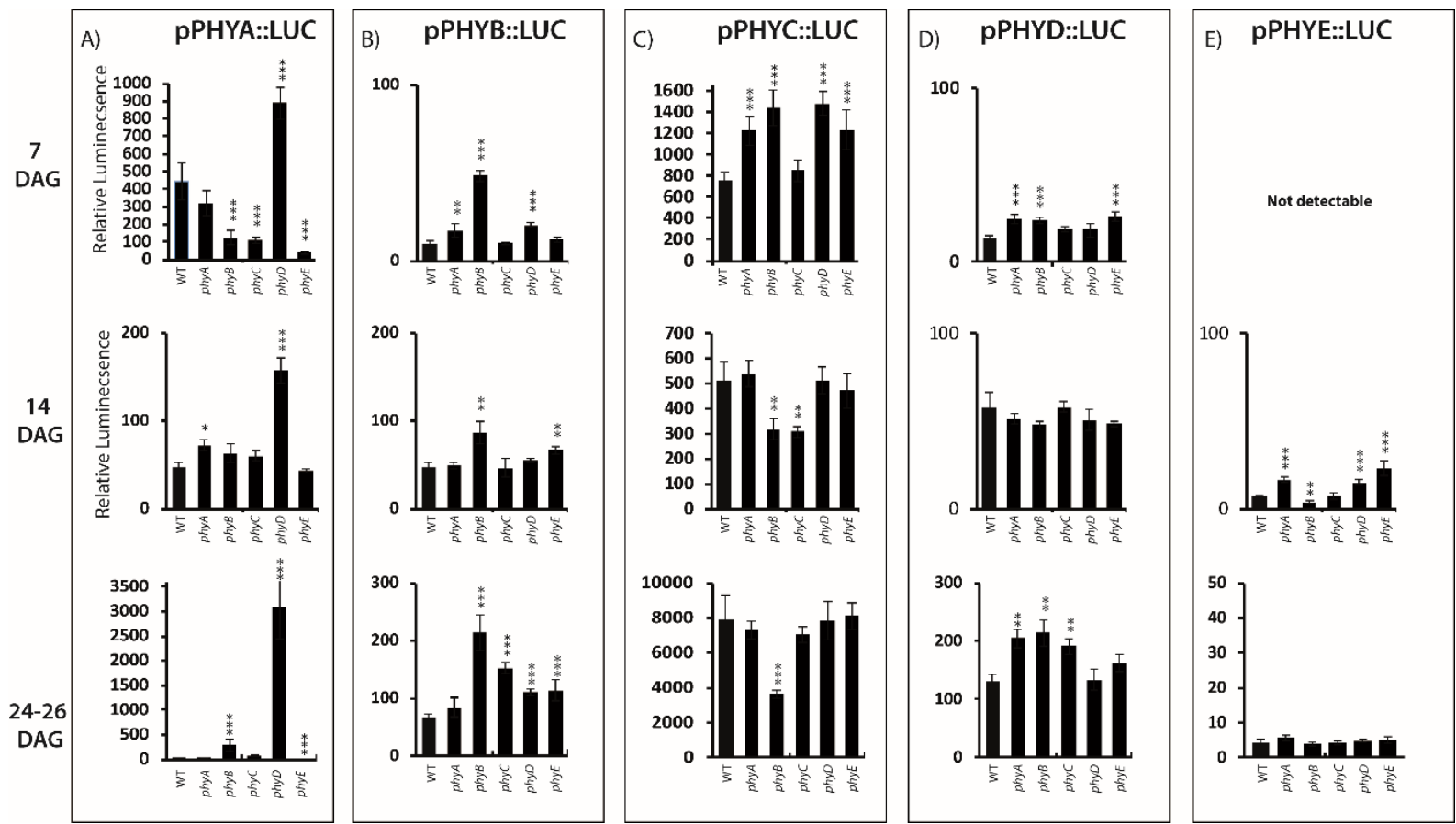

Figure-2. $\mathrm{pPHY}: \mathrm{LUC}$ activity at $\mathrm{ZT}=3 \mathrm{hr}$ in plants grown under different developmental stages. Plants were grown in growth cabinets under fluorescent WL and sprayed with substrate luciferin (1 $\mathrm{mM}$ ) one day and one hour before imaging at $11 \mathrm{am}(\mathrm{ZT}=3 \mathrm{hr})$. Plants were imaged at 7 or 14 days after germination from White light (WL). Plants were pre-grown for 25 days in growth cabinets under fluorescent WL and sprayed with substrate luciferin $(1 \mathrm{mM})$ one day before placing in LUMINATOR regime. The light intensity during 2 hour ramping at start-day and end-day is $33 \mu \mathrm{mole} \mathrm{m}^{-2} \mathrm{~s}^{-1}$ and during the remaining hours of the photoperiod $90 \mu \mathrm{mole}$ $\mathrm{m}^{-2} \mathrm{~s}^{-1}$. After adaptation for one day in LUMINATOR, LUC activity images were obtained every half hour $(7 \mathrm{~min}$. exposure) for a full diurnal cycle under mixed LED light (Figure S2). A: PPHYA:LUC in WT and five phytochrome mutants; B: pPHYB:LUC in WT and five phytochrome mutants; C: PPHYC:LUC in WT and five phytochrome mutants; D: pPHYD:LUC in WT and five phytochrome mutants; E: PPHYE:LUC in WT and five phytochrome mutants (not detectable at 7 days). The relative LUC activity was quantified in ImageJ. Number of replicate plants for each reporter line: $\mathrm{N}=9$ for $7 \mathrm{DAG}, \mathrm{N}=9$ for $14 \mathrm{DAG}$ and $\mathrm{N}=6$ for rosette plants. Error bars represent mean $\pm \mathrm{SE}$. Error Bars with symbols $\left({ }^{*} ;{ }^{* *} ;{ }^{* * *}\right)$ indicate a significance to compare WT respective to $p$-value $<0.05 ;<0.01$; $<0.001$.

To determine the diurnal pattern in pPHY:LUC activity in WT and phytochrome mutants, the plants were grown in under mixed LED light $(R+F R+B)$ in a custom built cabinet for LUC activity imaging named LUMINATOR. In LUMINATOR LED lights are used instead of fluorescent lights, because fluorescent lights have a strong after-glow in the dark, which interferes with LUC activity measurements. Intensities of R, FR and B LED light in LUMINATOR are adjusted for the closest match to a full natural WL spectrum. Light conditions during the 12 hour photoperiod include a two hour ramping with half-light intensity and decreased $R: F R$ (R:FR=0,2 instead of $\mathrm{R}: \mathrm{FR}=0.8$ ) at start and end of each 12 hour photoperiod. This block ramping light regime crudely mimics the changing light conditions in morning and evening in a natural environment. 
The pPHY:LUC activity images were obtained every 30 minutes during a full diurnal light cycle $12 \mathrm{~L} / 12 \mathrm{D}$ for 25 day old rosette plants. The average relative LUC activity in each of the reporter lines was quantified (Figure S2). Similar as for plants grown under fluorescent WL (Figure 2), the pPHY:LUC activity at ZT=3hr in WT plants and phytochrome mutants grown under mixed LED is compared (Figure 2). Results show that for 25-day old plants, grown under mixed LED light, the interactions between the different PHY genes is again different from that in seedlings (compare interactions at 7,14 and 25 days, Figure 2), indicating that extend of genetic interaction between phytochrome genes transcription may depend on development and/or light condition. For instance, for PPHYA:LUC activity under WL in rosette plants there was little effect of the other phytochrome genes (except for PHYD), while under mixed LED lights pPHYA:LUC activity is affected by multiple PHY genes. The PHY genes are not only light regulated but also regulated by the circadian clock (Toth et al., 2001). The interactions at the transcription level of phytochrome gene activity in seedlings as observed in the different phytochrome mutants may therefore be explained in two ways: either individual phytochromes affect the amplitude of oscillations in other PHY gene transcription, or individual phytochrome mutations cause a shift in the phase of PHY gene expression relative to that in WT plants. However, we note that the diurnal oscillating activity of the PPHY:LUC reporters is not very strong in 25 day old plants (Figure S2).

\section{Strong induction of PHYB and PHYA by FR Light}

Experimental conditions for seedling growth analysis often include growth conditions using pure $\mathrm{R}, \mathrm{FR}$ or B LED lights. We therefore next determined the expression profile of the pPHY:LUC genes for one day under these artificial diurnal light conditions. For this, seedlings were pre-grown for 7 days under 12WL/12D in growth cabinets, pre-sprayed with luciferin and transferred to LUMINATOR for adaptation under mixed LED lights for one day and night. Subsequently, pPHY:LUC activity was measured in WT reporter plants under 12R/12D, $12 F R / 12 D$ and 12B/12D diurnal LED light regimes. These experiments were repeated when plants were 14-days old and when plants were 25-day old plants. Qualitatively the responses of the different PPHY:LUC reporters were the same at these three stages of development. Results of expression profiles for 14 day old plants are shown in Figure 3. Here we only discuss 
the strong effects on PPHY:LUC activity. Most pPHY:LUC reporters did not show strong response to the $\mathrm{R}$ photoperiod, except for PPHYC:LUC which is induced under R. Most remarkable is the strong and immediate upregulation of pPHYB:LUC under FR light, reaching a peak expression almost 10-fold higher then under R light. Expression of pPHYA:LUC is also upregulated by FR light but in a more gradual way, reaching a 6-fold higher expression at the end of the FR photoperiod compared to under R. Other phytochrome promoters were not induced by FR or showed a decline of expression under FR. During the night following FR, expression of PHYB and PHYA show an initial rapid decline. The PPHYC:LUC shows a transient increase in activity at the day-night transition following all photoperiods. Finally, under $B$ pPHYC:LUC and PPHYB:LUC show a transient induction of activity. We note that leaf hyponastic movement under the given light condition causes some of the fine structure in the LUC activity profiles. 


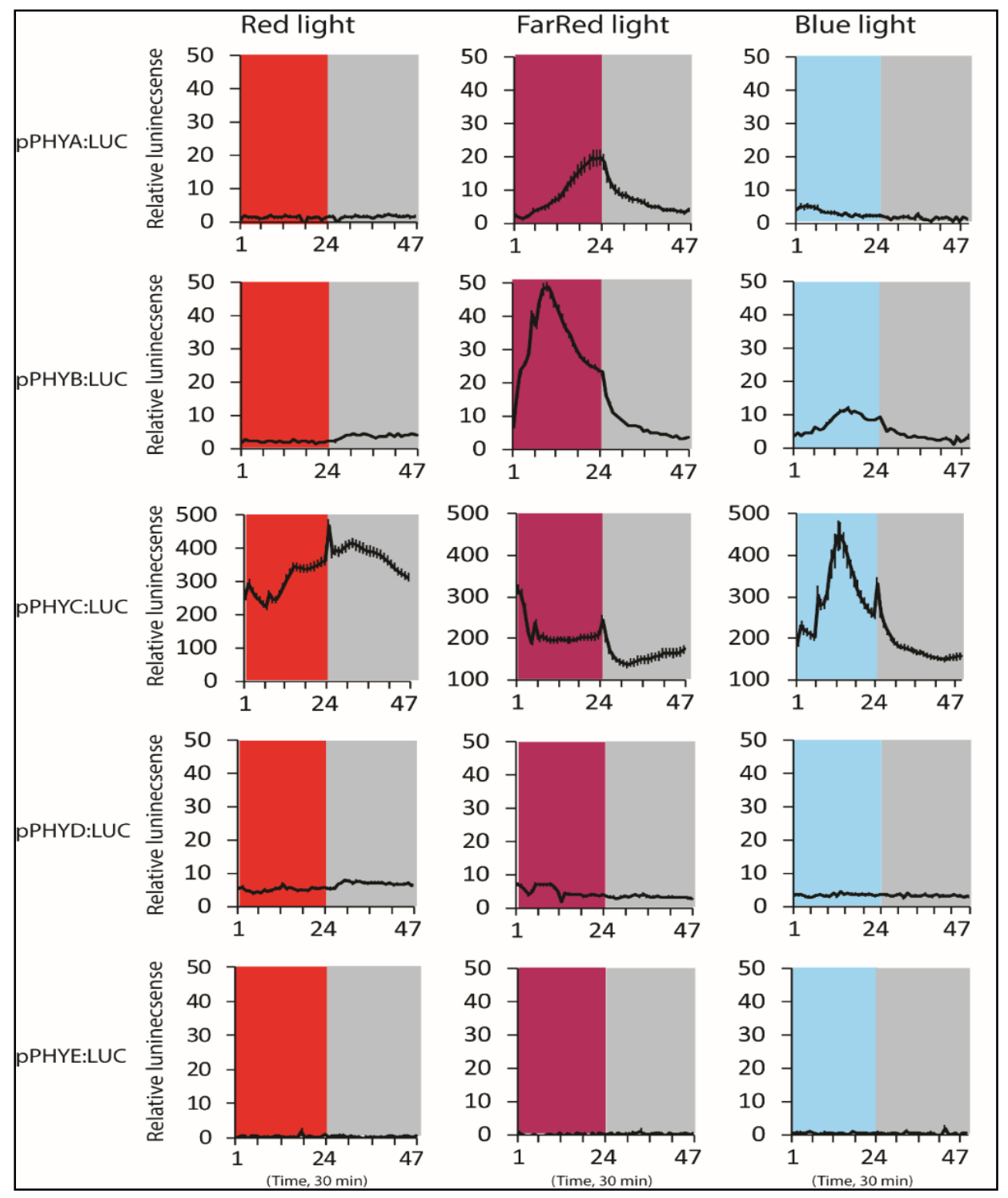

Figure-3. A diurnal expression profile of the PPHY:LUC reporters under diurnal 12R/12D, 12FR/12D and 12B/12D. Seeds were stratified and germinated in growth cabinets under diurnal fluorescent $W L$ (12L/12D). At 14 days after germination seedlings were sprayed with substrate luciferin (1 mM) and one day later placed in LUMINATOR for adjustment under diurnal $R+B+F R$ for one day. Subsequently plants were exposed to light regimes $12 R / 12 \mathrm{D}$, followed by $12 \mathrm{FR} / \mathrm{D}$ and finally $12 \mathrm{~B} / 12 \mathrm{D}$. Luciferin $(1 \mathrm{mM})$ solution was sprayed once per day. LUC activity images were obtained every half hour ( $7 \mathrm{~min}$. exposure) for each full diurnal cycle. The relative LUC activity was quantified in ImageJ and corrected for background signal. Number of replicate seedlings for each reporter line: $\mathrm{N}=6$. Error bars represent mean $\pm \mathrm{SE}$.

Transcription of PHYB under FR is not affected by PHYA but by PHYB and PHYE: PHYB and PHYE novel sensors of FR

The strong induction of pPHYB:LUC and pPHYA:LUC activity in WT plants by FR light suggests a transcriptional regulation by phytochrome signaling. In classical photobiology the PHYA is 
linked to FR light responses (Whitelam et al., 1993; Yanovsky et al., 1997; Fankhauser, 2001). To determine whether PHYA is responsible for the upregulation of PHYB gene activity under FR or whether any other phytochrome is involved in this strong induction, the PPHYB:LUC reporter activity was monitored in the different phytochrome mutant backgrounds in 14-day old seedlings grown under mixed light, R FR or Blue light (Figures S3). Figure 4 shows part of the results in which PHYB gene expression is significantly affected by other phytochromes. The

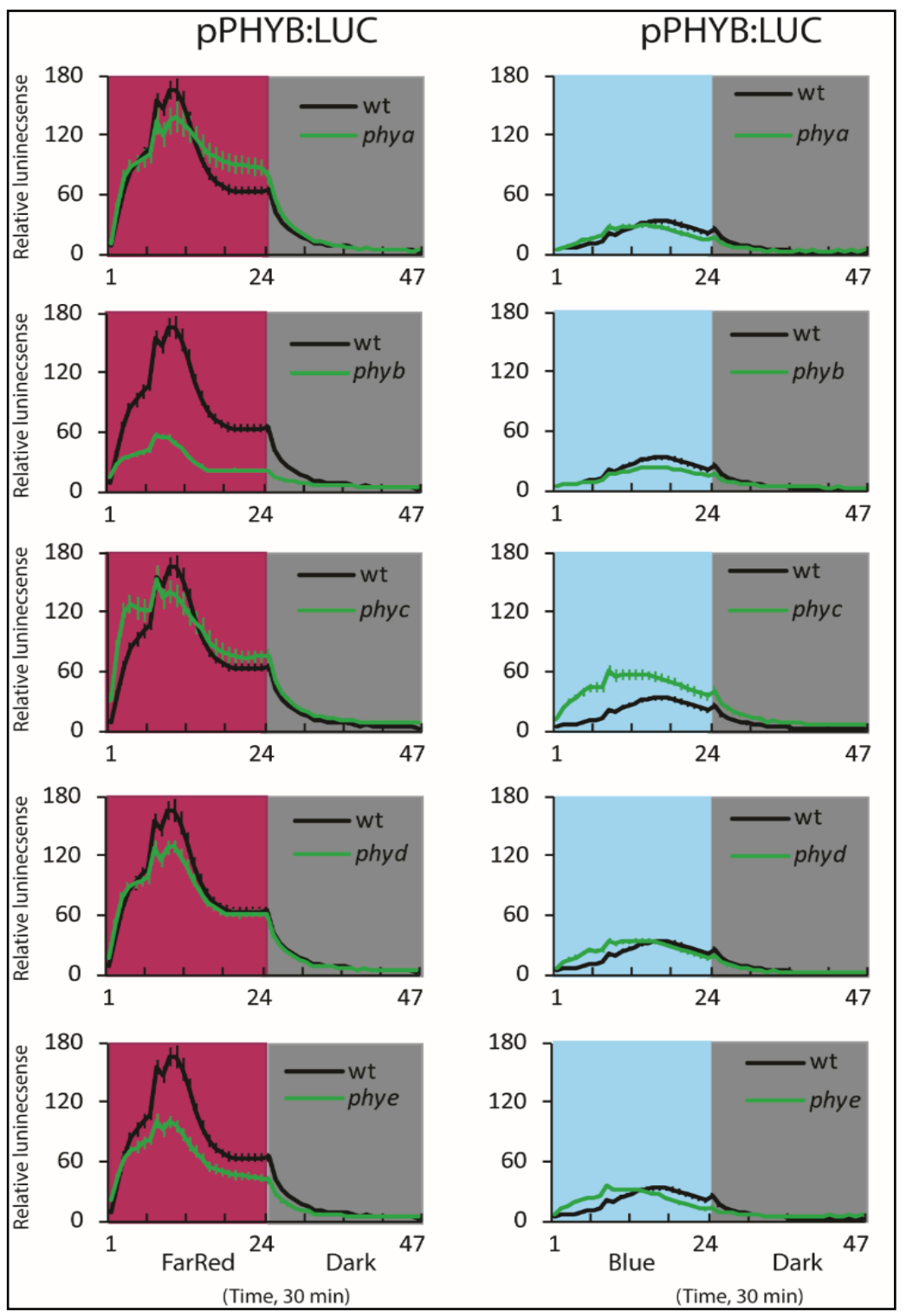
expression of pPHYB:LUC is strongly decreased under FR in the phyB mutant and slightly decreased in the phyE mutant background (Figure 4). This indicates that in the context of transcriptional regulation of PHYB gene expression PHYB and PHYE act as a FR sensor, while the classical FR sensor PHYA has little effect on PHYB gene activity under FR.

Figure-4. pPHYB:LUC activity in WT and phytochrome mutants in $\mathbf{1 4}$ day old seedlings under FR

or B. Seeds of the PPHYB:LUC reporter lines were stratified and germinated in growth cabinets under diurnal fluorescent $\mathrm{WL}$ (12L/12D). At 14 days after germination seedlings were sprayed with substrate luciferin (1 $\mathrm{mM})$ and one day later placed in LUMINATOR for adjustment under diurnal $R+B+F R$ for one day. Then plants were exposed to light regimes of 12mixed/12D, 12R/12D, 12FR/D and finally 12B/12D. Luciferin (1 mM) solution was sprayed once per day. LUC activity images were obtained every half hour ( $7 \mathrm{~min}$. exposure) for each full diurnal cycle. The relative LUC activity is quantified in ImageJ and adjusted for background signal. Number of replicate seedlings for each reporter line: $\mathrm{N}=16$. Error bars represent mean $\pm \mathrm{SE}$. All results are shown in Figure S3. Here only results for pPHYB-LUC in WT and phy-mutants under FR and B are shown. 


\section{Feedback interaction of phytochromes on PHYB gene expression is function of light quality}

Expression of PHYB in the phyB mutant background under mixed or $\mathrm{R}$ light is increased, but decreased under FR and B LED light (Figure S3). This shows that the effect of PHYB on its own expression is dependent on the light conditions and may switch from a repressor interaction (under mixed and R light) to activator interaction (under FR and B) (Figure S3 and Figure 4). Similar results were obtained for 7-day and 14-day old seedlings (not shown). The diurnal pattern of PHYB promoter activity under the different light regimes indicate that the phase of pPHYB:LUC activity is dependent on the light conditions (phase of pPHYB:LUC in WT under

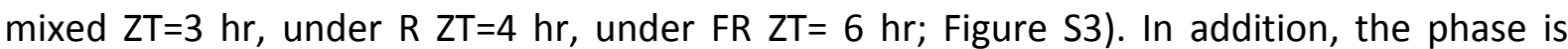
dependent on the phytochrome mutant background (phase of pPHYB:LUC under B in WT ZT=3 $\mathrm{hr}$, in phyb mutant $\mathrm{ZT}=7 \mathrm{hr}$, in phyC mutant $\mathrm{ZT}=2 \mathrm{hr}$; Figure 4).

PPHY:LUC expression as function of different artificial "shade" conditions in rosette plants $(R>R+F R$ and $R+F R>R$ )

To investigate the phytochrome gene expression as function of different shade light conditions during the day, we measured the different PPHY:LUC reporter activities in WT rosette plants under varying R:FR light conditions. For this reporter plants were grown for 25 days in growth cabinets under 12WL/12D. Subsequently, the five WT pPHY:LUC reporter plants were placed in LUMINATOR to adapt for two days to diurnal mixed LED light (R,B,FR). After the night of the second day, the photoperiod was started using $R$ light with low level of $F R(R: F R=8)$. Subsequently, every 3 hours the $R$ level remained the same, but dosage of $F R$ was increased going from $R: F R=8$ to $R: F R=1$, to $R: F R=0.5$ and finally ending the day with 3 hours of $R: F R=0.2$, which mimics deep shade conditions. After the night following these 4 blocks of increasing shade light conditions, the next day, the same blocks of R+FR LED light were given in reverse order, starting the day with $\mathrm{R}: \mathrm{FR}=0.2$ and ending the day with $\mathrm{R}: \mathrm{FR}=8$. Under these conditions the different pPHY:LUC reporters show different responses (Figure 5). First of all, a strong transcriptional response to $3 \mathrm{hr} R: F R=8$ is absent for PHYA and PHYB. In the subsequent 3 hours, when FR levels are further increased to R:FR= 1, pPHYB:LUC shows an direct transcriptional response, while for PHYA and PHYC a transcriptional response starts near the 
end of this three hour light treatment (Figure 5). In contrast PHYD expression is down regulated near the end of this light treatment. This is consistent with our discovery that PHYD is a suppressor of PHYA and suggests that the upregulation of PHYA during the rest of the day is caused by the downregulation of PHYD during the rest of the day. However, the following day when light treatments are given in reverse order, pPHYD:LUC activity shows an increase at the end of the first $R: F R=0.2$ light treatment, which is not mirrored by a decline in pPHYA:LUC activity. During the night, expression of PHYA, PHYB and PHYC decline with different initial rates, but expression at the end of the night remains well above that seen under mixed, $R$ or B LED light. The decline at night after $R+F R$ is different from the decline in PHY gene expression after pure FR, during which expression rapidly declines to "normal" levels as seen under WL, mixed LED or B (Figure 3). The following day light treatment start with 3 hours of deep shade conditions ( $R: F R=0.2$ ), similar to the last 3 hours of the previous day. For PHYA, PHYC and PHYD this results only in a small transient transcriptional upregulation of expression. However, for PHYB there is an immediate and continuous upregulation of gene transcription. During the following three phases of the light treatment, when the FR component is step wise reduced, PHYA shows a small increase in expression, reaching a plateau during the last two light treatments. For PHYB the expression reaches a plateau during $R: F R=0.5$, after which expression declines under $R: F R=1$ and $R: F R=$ treatment. $P H Y C$ and $P H Y D$ expression decreases when R:FR increases. Overall, the results suggest again a strong response of PHYB and PHYA gene expression to FR light conditions, but the order in which $R: F R$ light treatments are given influences the response. Subsequently it was tested what the role of individual phytochromes is in the response of the pPHY:LUC reporters to different ratio's of R:FR. 


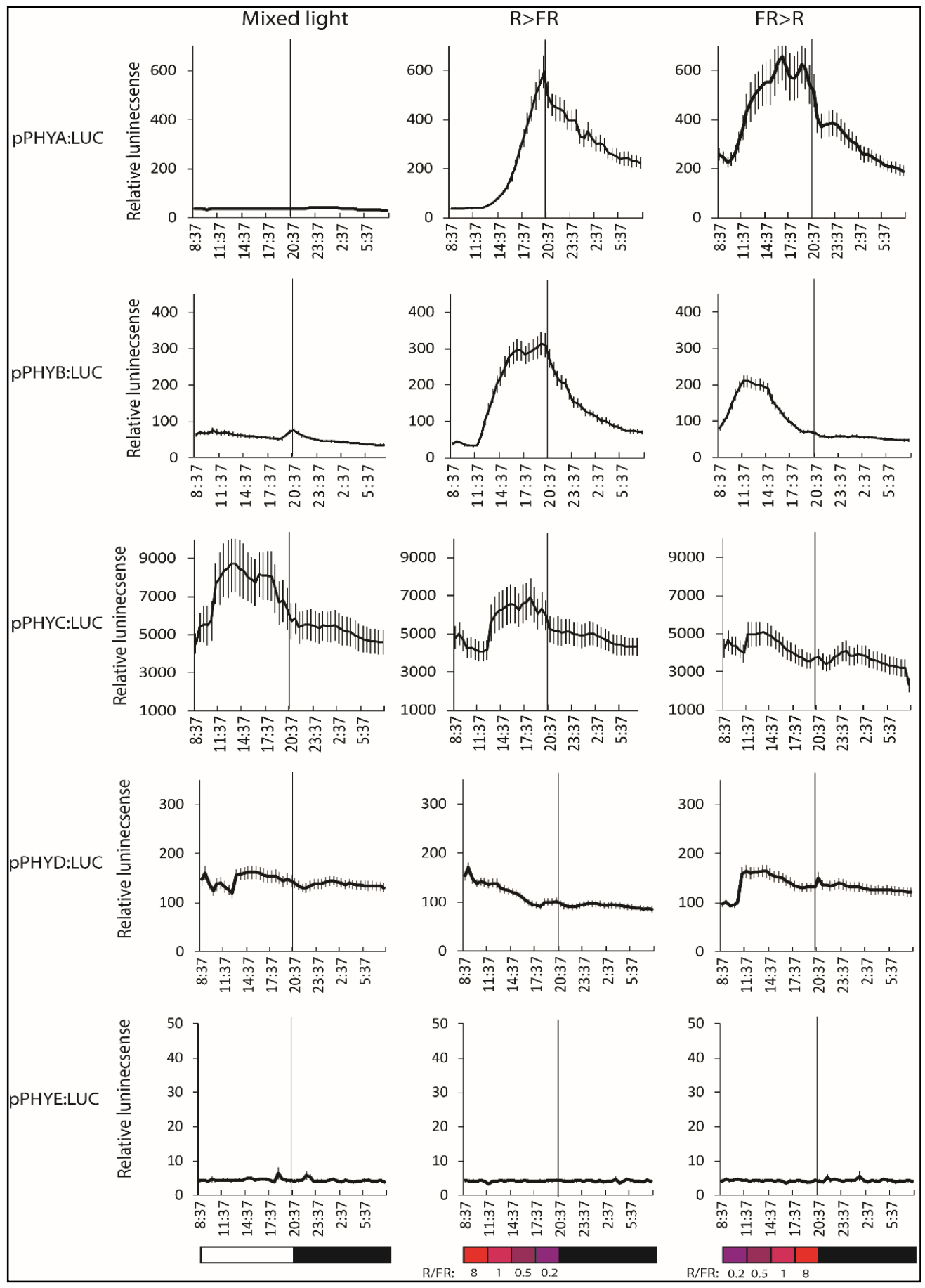

Figure-5. pPHY:LUC activity in WT rosette plants in response to changing R:FR ratios. pPHY:LUC in WT plants were grown in growth cabinets under diurnal fluorescent WL (12L/12D) for 25 days. Reporter plants were sprayed with substrate luciferin $(1 \mathrm{mM})$ and one day later placed in LUMINATOR for adjustment under diurnal mixed $R+B+F R$ for one day. Subsequently rosette plants were exposed to $R$ light with increasing levels of $F R$ (in blocks of 3 hours), resulting in R:FR ratio's of $8,1,0.5$ and 0.2 . After the following night plants were exposed to the reverse light regime. Luciferin $(1 \mathrm{mM})$ solution was sprayed once per day. LUC activity images were obtained every half hour ( 7 min. exposure) for each full diurnal cycle. The relative LUC activity was quantified in Image J and corrected for background signal. At least 7 replicate rosette plants were used for each reporter line. Error bars represent mean $\pm \mathrm{SE}$. The vertical line indicates the day to night transition. 


\section{PHYE is required for PHYA response under R/FR}

To determine if any of the five phytochromes is specifically involved in the strong response to different R:FR ratios we measured the PPHY:LUC reporter activities in all different phytochrome mutant backgrounds. The same light regimes as used in the experiment shown in Figure 5 were used: 3 hours $R: F R=8,3$ hours $R: F R=1,3$ hours $R: F R=0.5$ and ending with 3 hours of R:FR=0.2 and reverse order of these light regimes the following day. An overview of all the results is given in Figure S4A-E. Here only the big effects on PHYA and PHYB expression are presented (Figure 6A-B). The expression profile of pPHYA:LUC most deviating from that in WT is the expression in the phyD mutant background. Going from R:FR=8 to R:FR=0.2 the expression of PHYA in phyD mutant shows a similar profile as in WT, but at much higher level. However, when going from R:FR=0.2 to R:FR=8 the PHYA expression in WT shows an increase in activity, while in the phyD mutant a response is lacking (Figure 6A). Moreover, the upregulation of PHYA under increasing levels of FR is absent in the phyE mutant background (Figure 6B), suggesting that PHYE is a strong sensor of FR light in the regulation of PHYA gene expression. In contrast, the phyA mutation had only a weak effect on FR-induction of pPHYA:LUC (Figure S4). For PHYB the activity is most affected by PHYB itself, as PHYB is required for full expression level of pPHYB:LUC under increasing FR light conditions (Figure S4). The effect of PHYC, PHYD and PHYE on PHYB expression is conditional: they have little effect on pPHYB:LUC activity under the light regime going from high R:FR to low R:FR, but these phytochromes act as suppressor of PHYB expression when light changes from low R:FR to high R:FR (Figure S4). 


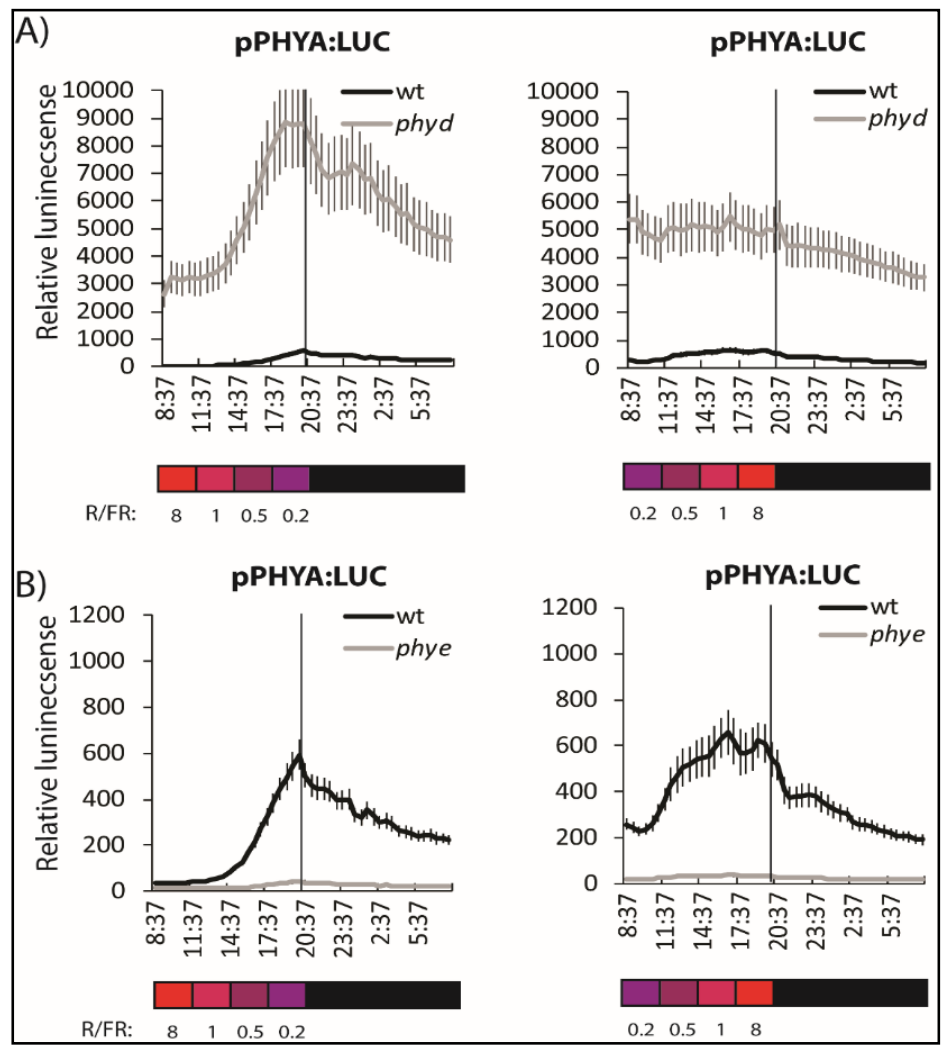

Figure-6. pPHYA:LUC activity in WT and phyD and phyE mutant in response to changing R:FR ratios. PPHYA:LUC in WT, phyD and phyE plants were grown in growth cabinets under diurnal fluorescent WL (12L/12D) for 25 days one day later placed in LUMINATOR for adjustment under diurnal mixed R+B+FR for one day. Subsequently rosette plants were exposed to $R$ light with increasing levels of FR (in blocks of 3 hours), resulting in R:FR ratio's of $8,1,0.5$ and 0.2 . After the following night plants were exposed to the reverse light regime. Every day once at 11AM plants were sprayed with substrate luciferin $(1 \mathrm{mM})$ solution. LUC activity images were obtained every half hour (7 min. exposure) for each full diurnal cycle. The relative LUC activity was quantified in Image J and corrected for background signal. Number of replicate seedlings for each reporter line: $\mathrm{N}=7$. Error bars represent mean \pm SE. A: pPHYA:LUC activity in WT, phyD. B: pPHYA:LUC activity in WT and phyE. Note that for activity in phyD mutant the scale of relative LUC activity was adjusted. Black: PPHYA:LUC in WT, grey: PPHYA:LUC in phy mutant. (Expression of all pPHY:LUC reporter lines in WT and phy-mutants under changing $R: F R$ is given in Figure $S 4)$.

\section{Validation of the pPHY:LUC reporter results by selected qPCR}

The output of the LUC reporter system is not only a function of the promoter driving LUC transcription, but also a function of luciferin substrate availability and the physiology of the cell, which may affect required oxygen and ATP levels (Marques and Esteves da Silva, 2009). 
The full dynamic analysis of PHY gene activity under many different conditions would need validation by $\mathrm{qPCR}$ of too numerous time and development samples. Therefore we concentrated on those conditions and developmental stages that show the most novel PHY interactions as revealed by the pPHY:LUC reporters, for validation of endogenous PHY mRNA levels by qPCR. The qPCR analysis confirm the upregulation of PHYA under FR as shown by pPHYA:LUC activity. However, quantitatively the induction of the PHYA-LUC reporter is much stronger than as observed for the endogenous PHYA gene (Figure 7A). Expression of the endogenous PHYA gene is not significantly higher in phyD compared to WT for seedlings grown under $\mathrm{R}$ light (Figure 7A), while the pPHYA:LUC reporter is twice as active in the phyD mutant at this stage (Figure 1). However, expression of the endogenous PHYA gene under FR is significantly higher in the phyD mutant (Figure 7A), indicating that PHYD is involved in the suppression of PHYA transcription. The qPCR analysis also confirms that the upregulation of PHYA expression under FR is reduced in the phyE mutant background, confirming that PHYE is

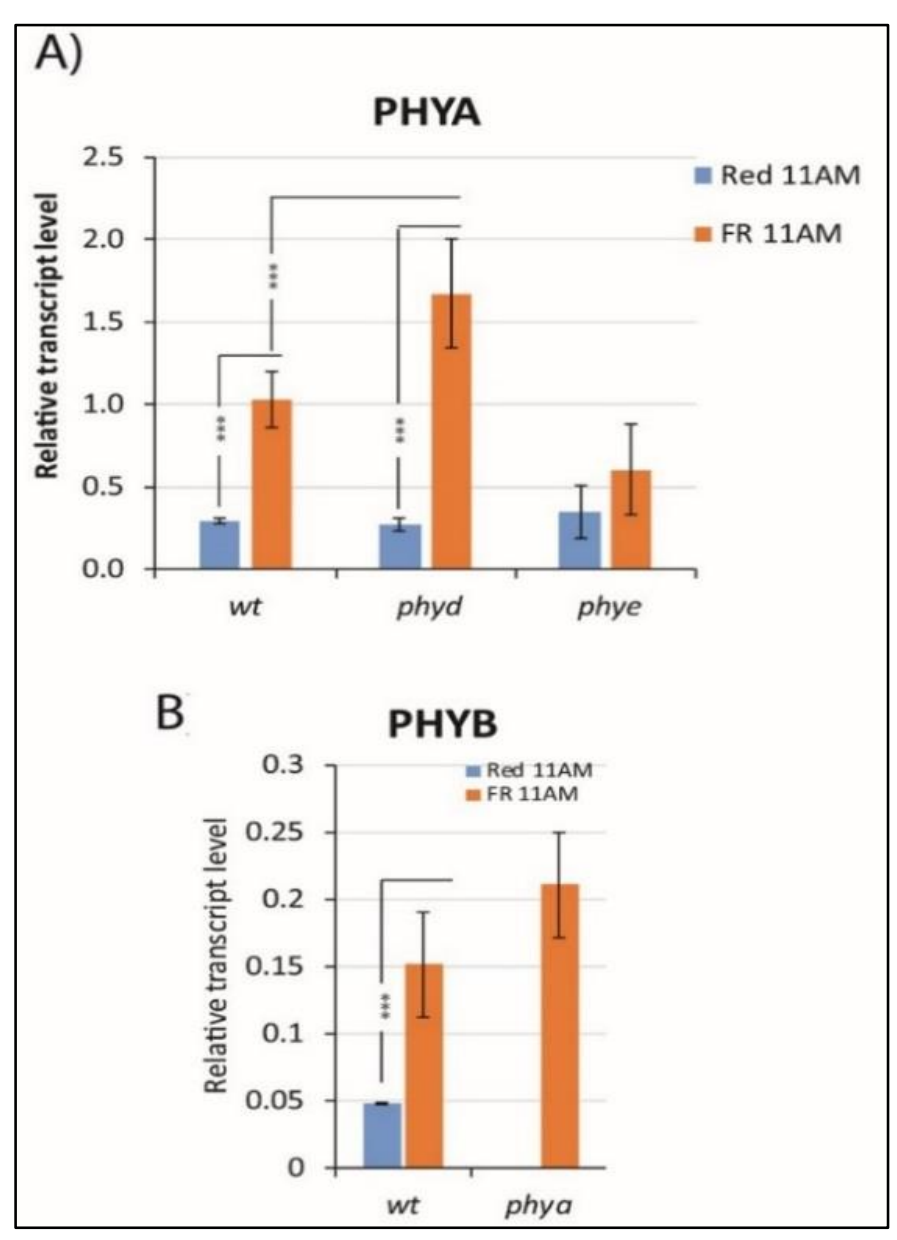

required for the FR response of PHYA transcription (Figure 7A). The induction of $P H Y B$ transcription under $F R$ is also confirmed by qPCR, but like for the PHYA gene, quantitatively the induction of the PPHYB:LUC reporter is much stronger than the transcriptional induction of the endogenous $P H Y B$ gene (Figure 4 and Figure 7B).

Figure 7. qPCR analysis of PHYA and PHYB gene expression. (A) Relative expression of PHYA in wt, phyD and phyE mutant under $\mathrm{R}$ $\mathrm{ZT}=3 \mathrm{hr}$ and $\mathrm{FR} \quad \mathrm{ZT}=3 \mathrm{hr}$. and (B) relative expression of PHYB in wt and phyA mutant under $\mathrm{R} Z \mathrm{ZT}=3 \mathrm{hr}$ and $\mathrm{FR} Z \mathrm{ZT}=3 \mathrm{hr}$. $\mathrm{qPCR}$ data is based on three biological replicates from RNA isolated from 14 day old seedlings. Significant differences are indicated by $* * * \quad(p-$ value<0.05). 


\section{PIF4 is involved in the induction of PHYB expression under FR}

To determine which transcription factors may be involved in the FR-induction of the PHYB and PHYA genes, the promoters of all five PHY genes were analysed for putative transcription factor binding sites using PLAZA 3.0 Dicots (Proost et al., 2015). This analysis shows that no specific binding sites are present in the promoters of PHYA and PHYB that may link to the induction under FR (Table S3). Multiple G-box binding sites are presented in the PHYB promoter and a single G-box is present in the PHYD promoter (Table S3). G-box is not present in the promoter the other PHY genes. The brassinosteroid activated transcription factors BZR1/BES1 can bind to the G-box motif and BZR1 is a known suppressor of PHYB expression (Sun et al., 2010). PIF4 is another transcription factor known to bind to G-box and the combined action of BZR1 and PIF4 are known to regulate transcription of genes containing a G-box like YUCCA8 (Sun et al., 2012). Since both the PHYB and PHYD promoter contain G-box, the activity of the pPHYB:LUC and PPHYD:LUC reporter was tested in a pif4-2 mutant background to determine if PIF4 is involved in expression of these genes. For this the pPHYB:LUC and pPHYD:LUC reporter plants were crossed to pif4-2 and from F2 progeny the plants homozygous for the pif4-2 mutation and homozygous for the pPHYB:LUC or PPHYD:LUC reporter gene were selected to further investigation. Expression of pPHYB:LUC or pPHYD:LUC in WT and pif4-2 was measured in two week old seedlings under mixed, R, FR, B and R/FR ratio light conditions. Results shows that PPHYB:LUC is not changed in pif4-2 under mixed light, $\mathrm{R}$ or B, while pPHYB:LUC is significantly lower in pif4-2 compared to WT under pure FR (Figure 8A-D). The strongest effect of PIF4 on the pPHYB:LUC activity is observed at different R/FR condition (Figure 8E-F), indicating that under these conditions PIF4 is responsible for about half of the PHYB gene activity. Combined, these results indicate a role for PIF4 in expression of PHYB that is FR light dependent. In contrast, pPHYD:LUC activity was not affected in the pif4-2 mutant background under any of the light conditions tested, indicating that the G-box in the PHYD promoter is not a target of PIF4 (Figure S5). 


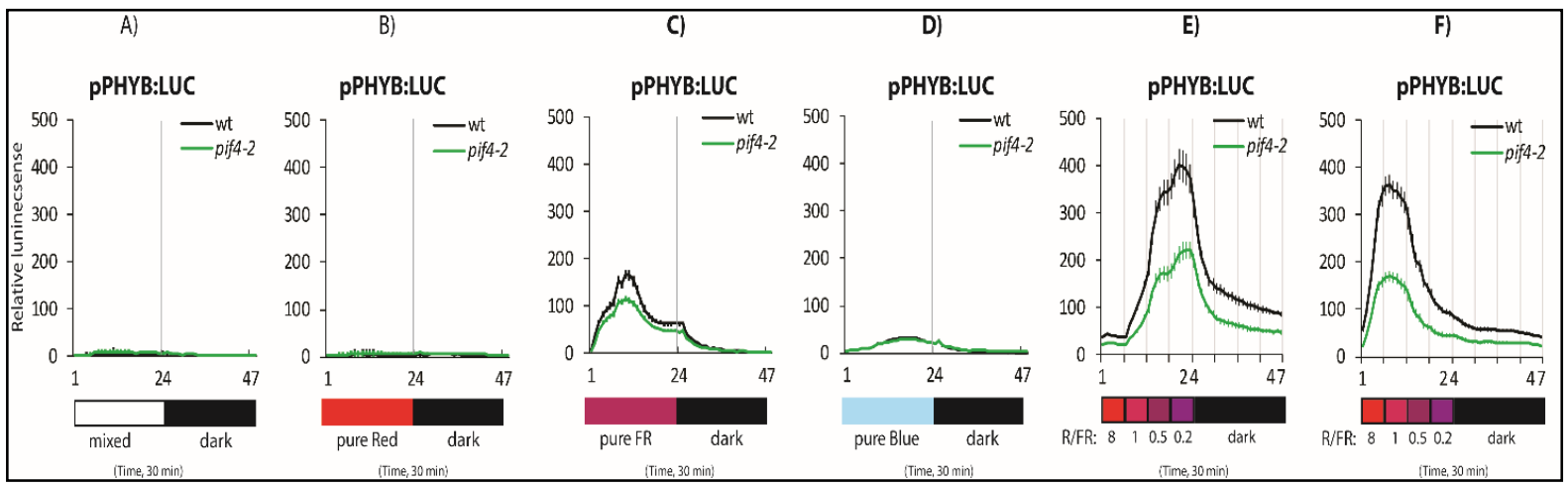

Figure 8. pPHYB:LUC expression under FR depends partly on PIF4. pPHYB:LUC reporter in WT and pif4-2 mutant backgrounds were stratified and germinated in growth cabinets under diurnal fluorescent WL (12L/12D). At 7 days after germination plants were sprayed with substrate luciferin $(1 \mathrm{mM})$ and one day later placed in LUMINATOR for adjustment under diurnal mixed R+B+FR for one day. Next three days, the light changed to pure $R, F R$ and $B$ light respectively (A-D). For $E$ and $F$ figures, seedlings were exposed to $R$ light with increasing levels of FR (in blocks of 3 hours), resulting in R:FR ratio's of $8,1,0.5$ and 0.2 . After the following night plants were exposed to the reverse light regime. LUC activity images were obtained every half hour ( 7 min. exposure) for each full diurnal cycle. The relative LUC activity was quantified in ImageJ. Background value subtracted from average of observed value (O-B). Number of replicate seedlings for each reporter line: $\mathrm{N}=11$. Error bars represent mean \pm SE.

\section{Discussion}

\section{Complex transcriptional regulation of PHY genes}

The sessile nature of plants requires sophisticated adaptation mechanisms to fluctuating environmental light conditions. For this plants have evolved several photoreceptors of which the phytochromes consist of the largest gene family. In Arabidopsis phytochrome signalling has been extensively investigated (Franklin and Quail, 2010). Phytochrome holoproteins are synthesised in their inactive phy ${ }^{\mathrm{Pr}}$ form, are activated by $\mathrm{R}$ light to phy ${ }^{\mathrm{Pfr}}$, and can be inactivated again by FR light to phy ${ }^{\mathrm{Pr}}$. Phytochrome activity under different light conditions has mainly been studied for signalling downstream of phy ${ }^{\mathrm{Pfr}}$, while the input of phytochrome protein level is usually not considered. However, the level of active phytochrome is both a function of the total phytochrome protein pool and the equilibrium between the pool of active phy ${ }^{\mathrm{Pfr}}$ and inactive phy ${ }^{\mathrm{Pr}}$, which is determined by R:FR ratio. For the total phytochrome signalling potential the transcription of the phytochrome genes is therefore an important input factor. Here we have used PPHY:LUC reporter plants to study the PHY gene transcriptional activity at different stages of development and under different light conditions. 
These studies have shown that under artificial fluorescent WL light conditions (the closest to natural light used in this study) multiple genetic interactions between the PHY promoter activities are observed at the seedling stage which change over the course of development (Figure 2). Moreover, regulation of phytochrome promoter activity is also a function of light quality (Figure 3 and 5). This flexibility seems to be related to the changing way in which phytochromes affect each other's promoter activity as function of development and as function of light quality.

\section{Quantitative differences between PHYA and PHYB reporters and endogenous PHYA and PHYB expression under FR}

Validation of the PHY-LUYC reporter results shows that for the FR induction of pPHYA:LUC and PPHYB::LUC, the results for the endogenous PHYA and PHYB gene are quantitative similar (Figure 7), but qualitatively the induction under FR is much lower for the endogenous PHYA and PHYB genes. This may be explained in different ways. For the qPCR analysis the mRNA is isolated from whole seedling (cotyledons plus hypocotyl), while for the LUC reporter seedlings mostly activity in the cotyledons is measured. Alternatively, the response of pPHYA:LUC and PPHYB:LUC in the selected reporter lines may not be representative for the PHYA and PHYB expression under FR. All PHY:LUC reporter lines have been selected as being representative for activity displayed under WL conditions. It may be necessary to re-screen independent PHYA:LUC and PHYB:LUC reporter transformants for the response to FR to determine if the strong response to FR is unique of the chosen PHYA:LUC and PHYB:LUC reporter lines, or a shared feature of all independent transformants expressing PHYA:LUC or PHYB:LUC. When the strong induction by FR is displayed in all independent PHYA:LUC and PHYB:LUC reporters, while induction of the endogenous gene is much more reduced, this could be indicative of additional regulatory elements in the introns of PHYA and PHYB. The qualitative differences in results may also be explained if the PHYA and PHYB mRNA have lower intrinsic stability under FR compared to the LUC mRNA under FR. 
PHYD is a constitutive suppressor of pPHYA:LUC, but conditional suppressor of endogenous PHYA

One of the strongest and consistent interactions these studies with the PHYA:LUC reporter have uncovered is the suppression of PHYA:LUC transcription by PHYD and suppression of PHYD by PHYA (Figures 2). However, for the endogenous PHYA gene the suppressive interaction with PHYD is only observed under FR light (Figure 7A). We note that reciprocal repression of PHYA and PHYD is consistent with the complementary expression profiles of PHYA and PHYD in developing and dry seeds (low PHYA, high PHYD), and imbibed seeds (high PHYA and low PHYD) (Toufighi et al., 2005). The function of PHYD in developing seeds thus may be to limit PHYA expression. A higher expression level of PHYA in the phyD mutant background under conditions with increased FR may also relate to some of the phenotypes that have been described for the Arabidopsis phyD mutant (Christians et al., 2012; SanchezLamas et al., 2016). PHYD can form a homodimer and heterodimers with PHYB, PHYC and PHYE. None of the mutants phyB, phyC or phyE show a strong effect on pPHYA:LUC expression (Figure 2), suggesting that it may be the combined loss of PHYD homodimers and heterodimers that are responsible for the strong upregulation of PHYA expression in the phyD mutant. Future analysis will have to show how PHYA expression is affected in double and triple mutants of PHYB,C and $E$.

\section{FR induction of PHYA and PHYB reporter expression is a novel FR-HIR response}

The classical high irradiance response (HIR) of Arabidopis is characterized by the suppression of hypocotyl elongation. Both PhyA and PhyB are involved in this HIR response (Quail et al., 1995), but phyB is mostly responsible for HIR under continuous Red (cR) light (R-HIR) (Nagatani et al., 1991; Reed et al., 1993) and phyA predominantly for the HIR responses under cFR light FR-HIR (Hartmann, 1967; Nagatani et al., 1993; Parks and Quail, 1993; Whitelam et al., 1993; Casal et al., 2014; Possart et al., 2014). One of the most remarkable results of these studies is the strong induction of PHYA promoter activity under FR light, which may be considered as a novel FR-HIR response. However, not PHYA but PHYE is involved in the FR-HIR induction of PHYA gene activity (Figure 6, Figure 7). Phytochromes are synthesized in the inactive Pr form, 
which absorbs maximally in red light. When Pr absorbs $\mathrm{R}$ light it changes into the active Pfr form, which has its maximum absorbance in FR. Absorption of FR by Pfr back-converts the molecule into Pr. However, due to the partial overlap between Pr and Pfr absorption spectra, far-red light is able to transform a small proportion of the Pr molecules into Pfr. Therefore, at very high PHY expression levels, there could still be an effective PHY signaling under FR due to the large phy protein pool. However, this does account for the specific action of PHYE under FR light, as PHYE expression is extremely low compared to any of the other PHY genes. Phytochromes are classified as either Type I, which are activated by far-red light, or Type II that are activated by red light (Li et al., 2011), although phytochrome Type I and Type II may also be defined by the phytochrome protein stability in light. For Arabidopsis only PHYA has been classified as a Type I phytochrome as it is responsible for many FR light induced responses and is instable in the light. Contrary to the five PHY genes in Arabidopsis, rice has only a PHYA, PHYB, and PHYC. Presumably, the PHYA, PHYB and PHYC were already formed before the formation of gymnosperms, as both monocotyledons and dicotyledons contain representatives of PHYA, PHYB, and PHYC. In dicotyledonous plants, duplications of the PHYB progenitors resulted in the PHYE subfamily and, specifically in Arabidopsis, another duplication event of PHYB resulted in PHYD (Clack et al., 1994). In contrast, grasses lack the PHYD and PHYE members of the PHYB subfamily. While the PHYC in Arabidopsis is a type II phytochrome, in rice, phyC mediates FR-HIR de-etiolation and therefore could be considered a Type I phytochrome (Takano et al., 2005). With the extension of FR-HIR responses beyond seedling de-etiolation to PHY gene expression under FR, the classification of Arabidopsis PHYE as type II phytochrome may need reconsideration.

Also the PHYB reporter activity is strongly induced under FR. The FR-HIR for PHYB expression under $\mathrm{FR}$ is reduced $40 \%$ in the phyE mutant, but about $70 \%$ reduced in the phy $B$ mutant (Figure 4). This identifies PHYE and PHYB as factors in the FR-HIR induced PHYB expression under FR. We note that the activity of PHYB on its own expression reverses depending on the light conditions: under mixed light and R LED PHYB suppresses its own expression, while under FR PHYB is required for the full induction response (Figure 5). This light dependent activity is also visible in the experiment with different R:FR light treatments, which shows that PHYB strongly suppresses its own gene expression under mixed LED light, but is required for the response to R plus added FR light (Figure S4). 
Different relative expression levels of PHY genes in rosette plants have consequences for dimer formation?

The analysis of pPHYC:LUC activity shows that PHYC is the most active phytochrome concerning the level at which it is expressed. At the seedling stages the PHYC expression is affected by PHYA, PHYB, PHYD and PHYE, but in mature rosette plants only PHYE has a significant effect on PHYC expression for plants grown under fluorescent WL (Figure 2). However, for rosette plants, grown under mixed LED, the PHYC expression is not much affected by PHYE. In contrast, for plants under mixed LED the expression of the PHYC reporter is strongly induced by PHYB. At present we assume that the genetic interactions in PHY reporter expression occur by co-expression of these PHY genes in the same cell and may therefore relate to the different phytochrome heterodimers that may be formed. Phytochrome protein binding studies have revealed that PHYC may form heterodimer with PHYB and PHYD and PHYC may not exist as homodimer (Clack et al., 2009). The relative high expression of PHYC compared to that of PHYB, PHYD and especially PHYE (which is expressed at very low levels), suggest that PHYB and PHYD may preferentially exist as heterodimer with PHYC and that PHYB/D and that PHYB/E heterodimers are only formed as minor components. Removal of PHYC from this pool of interacting phytochromes could therefore result in a substantial increase in the pool of PHYB/D and PHYB/E heterodimers. In this context it is remarkable that the strong induction of PHYB reporter activity under FR is strongly affected by PHYB but not PHYC or PHYD. This suggests that the induction of PHYB expression under FR is not mediated by $\mathrm{PHYB} / \mathrm{C}$ or $\mathrm{PHYB} / \mathrm{D}$ heterodimers, but mostly through other PHYB containing dimers (Hofmann, 2009).

\section{FR induced transcriptional regulation by PIFs requires light grown seedlings?}

The FR induced expression of PHY genes was demonstrated in seedlings that were pre-grown under white light and full grown plants. However, a FR transcriptional response of PHY genes is not observed for 3-day old dark grown seedlings (AtGenexpress light series). This indicates that the competence to respond to $\mathrm{FR}$ light is absent in dark grown seedlings. We speculate 
that the competence of light-grown seedlings to respond to FR may require fully developed plastids, which needs to be investigated in the future. Recently, it was shown that PHYA expression is a function of PIF (phytochrome-interacting factor) activity, specifically under short day conditions (Seaton et al., 2018). PIFs are members of the bHLH transcription factor family that connect light activated phytochrome Pfr to gene activation during germination, seedling de-etiolation, $R$ and $F R$ light responses and shade responses (Castillon et al., 2007). It has been shown that at the protein level PIF1, PIF3-5 and PIF7 interact with PHYB Pfr through an APB (active phytochrome B) domain, and that PIF1 and PIF3 can also interact with PHYA through an APA (active phytochrome A) domain (Khanna et al., 2004; Leivar et al., 2008; Shen et al., 2008). Analysis of the PHY promoter sequences shows that all PHY promoters have binding sites for circadian clock components (LHY, CCA1) (Table 2). In addition, all PHY promoters contain PIF1/PIF3 TF binding sites, while the promoters of PHYA, PHYB and PHYD also have PIF4/PIF5 binding sites (Oh et al., 2012; Sun et al., 2013) (Table 2)

The role of PIF proteins in PHYA transcription during short days raises the question whether these PIF proteins are also involved in the FR induction of PHYA transcription (Seaton et al., 2018). We note that during seedling establishment the PHYA protein levels are apparently not affected in the absence of PIF4 and PIF5 (Lorrain et al., 2009). Moreover, a four hour FR treatment of seeds germinated in the dark at four days after germination does not induce a transcriptional response for PHYA or PHYB (https://www.arabidopsis.org/portals/expression/microarray/ATGenExpress.jsp), even though PHYA and PHYB are expressed at this stage. However, this may be explained by no or low expression of PIF4 at this early stage of seed germination (vd Woude et al 2018 submitted) and other (not FR-responsive) factors than PIF4/5 acting in PHYA and PHYB promoter during early seedling establishment. The strong induction of PHY gene transcription under FR in our experiments may be related to the fact that (in contrast to PIF1 and PIF3) PIF4 and PIF5 proteins are not degraded in response to FR (Lorrain et al., 2009). However, the strong transcriptional response under FR is not simply explained through PIF stability, as the transcription of PHYA and PHYB rapidly drops in the dark, while supposedly PIF proteins are more stable and active in the dark.

In conclusion, we have established that FR induces both PHYA and PHYB and that this induction of PHYA is in part dependent on PHYE, while for PHYB the induction is dependent 
on PHYB and PHYE and on PIF4. It needs further investigation how a FR signal is translated into a higher transcription factor activity and how PHYE and PHYB can play a role under FR light conditions.

\section{Materials and Methods}

\section{Plant materials and growth conditions.}

Seeds of Arabidopsis thaliana T-DNA insertional mutant lines were obtained from the Nottingham Arabidopsis Stock Centre (NASC, University of Nottingham, UK). The following lines were used in our work: WT (Col-0), phyA-T(NASC: N661576), phyB-9 (Reed et al., 1993), phyC-2 (N66036), phyD (N676270), phyE-T (N671700), pif4-2 (SAIL_1288_E07). All phytochrome mutants are in Col-0 background. The phy T-DNA insertion mutants were validated as homozygous insertion mutant by PCR of genomic DNA using Salk T-DNA and gene specific primers (Table S2). In addition, specific light conditions were used to select homozygous mutant background in $\mathrm{F} 2$ after crossing with the different $\mathrm{PPHY}$ :LUC reporter lines in WT background. Screening of phy mutants in phenotype, the phyA plants complemented a reducing of germination in FR light, phyB and phyC mutants measured the hypocotyls length in R light, and long days (LD) light were used for phyD and phyE mutants were measured leaf length with comparison WT.

For Luminator experiments, seeds were sawn on MS-0.8\% agar plates (Murashige-Skoog medium $0.22 \mathrm{~g} / \mathrm{L}, 8 \mathrm{~g} / \mathrm{L}$ plant agar Duchefa), stratified in the dark for three days at $5^{\circ} \mathrm{C}$, after which they were sown on $4 \times 4 \times 4 \mathrm{~cm}$ rockwool blocks (Grodan, Roermond, The Netherlands) soaked in Hyponex nutrient solution (Unifarm, Wageningen, The Netherlands). Plants were pre-grown in a climate chamber $\left(12 \mathrm{hL} / 12 \mathrm{hD} ; 22^{\circ} \mathrm{C}\right.$; relative humidity $(\mathrm{RH})$ at $\left.65 \%\right)$. Directly before transfer to LUMINATOR, reporter plants were watered by soaking the rockwool blocks in Hyponex solution, which allows for growth for up to 6 days without additional watering. Growth conditions in LUMINATOR cabinet are described below.

Plasmid Constructs. Construction of the pPHY:LUC reporter genes using $2 \mathrm{~kb}$ upstream promoter fragments of either PHYA, B, C, D or PHYE is described in (Toth et al., 2001). Binairy 
vectors containing these reporter genes were kindly donated by the group of Prof. Nagy. For construction of the PIF4 reporter constructs the $2487 \mathrm{bp}$ intergenic region upstream of the PIF4 (At2g43010) start codon was amplified by PCR (primers listed in Table S2) using Q5 ${ }^{\circledR}$ HighFidelity DNA polymerase (New England Biolabs, Ipswich, MA, USA) and cloned by TOPO ${ }^{\circledR}$ Cloning reaction (Invitrogen, Carlsbad, CA, USA) into the PENTRTM TOPO ${ }^{\circledR}$ entry vector. To generate pPIF4::LUC expression constructs the entry vectors containing the PIF4 promoter sequences were recombined into the pGREEN-GW-Luc68 destination vector by LR recombination using Gateway ${ }^{\circledR}$ LR Clonase ${ }^{\circledR}$ II enzyme mix (Invitrogen, Carlsbad, CA, USA). The PPHY:LUC and pPIF4:LUC expression constructs were transformed to Agrobacterium tumefaciens (AGLO).

\section{Plant transformations and selection homozygous reporter plants}

Arabidopsis Col-0 plants were transformed by floral dip transformation (Zhang et al., 2006) and positive transformants were selected based on Luc activity. The seeds from self-pollinated individual $\mathrm{T}_{1}$ plants were harvested and sown on MS/Agar plate to determine the ratio of Luc activity in the $T_{2}$ generation. Lines were selected with 3:1 ratio of LUC activity, indicative of single insertion site. From these lines at least three reporter lines were selected which are homozygous for the PPHY:LUC reporter construct. For each of these homozygous PPHY:LUC reporter lines the LUC activity was determined at different stages of development and a representative reporter line for plants expressing either pPHYA:LUC, PPHYB:LUC, pPHYC:LUC, pPHYD:LUC or pPHYE:LUC was selected for further experiments.

Subsequently, the ultimately selected PPHY:LUC reporter line was crossed to the different phytochrome mutant plants and from the $\mathrm{T}_{3}$ progeny of this cross the plants homozygous for the PPHY:LUC reporter and homozygous for the phytochrome mutation were selected using LUC measurements for analysis of segregating LUC activity and different light (FR, R, LD) conditions to detect the phytochrome mutant phenotype. For instance, for screening for a homozygous phyA mutant background seeds were germinated under continuous Far-Red treatment as described by Chen et al (Chen et al., 2013). Under this condition, approximately $25 \%$ seeds do not germinate within 3 days, indicative of a phyA mutant background. None geminated seeds were subsequently transferred to white light. Eventually, for all progeny homozygous for the pPHY:LUC reporter, the homozygous phy-mutant genotype was 
confirmed by PCR. For measurements $\mathrm{T}_{4}$ plant homozygous for both the phytochrome mutation and the respective PPHY:LUC reporter were used.

\section{In planta LUC reporter activity measurements in LUMINATOR}

LUC activity in the different pPHY:LUC reporter plants was measured in a custom built LUMINATOR cabinet. The LUMINATOR contains a high performance PIXIS: 1024 CCD camera (Princeton Instruments, Roper technologies, Sarasote, FL, USA) fitted with a $35 \mathrm{~mm} f / 1.4$ Nikkor SLR lens (Nikon, Shinjuku, Tokyo, Japan) for imaging of bioluminescence in reporter plants. Reporter plants were pre-sprayed with the substrate $1 \mathrm{mM}$ D-luciferin (Promega, Fitchburg, WI, USA) one day before imaging to inactivate accumulated luciferase and make LUC activity dependent on ongoing promoter activity. For imaging plants are placed in LUMINATOR and for multiple day measurements sprayed daily with $1 \mathrm{mM}$ D-luciferin (Promega, Fitchburg, WI, USA) at $10 \mathrm{am}$. Plants were allowed to acclimate to conditions in LUMINATOR for remainder of the day and night. LUC activity images are taken every 30 minutes with an exposure time of 7 minutes. LED illumination during the photoperiod is switched off 30 seconds prior to imaging to allow for chlorophyll fluorescence decay. In addition, light from chlorophyll fluorescence of plants is blocked by using a ZBPB074 Bandpass Filter (Asahi Spectra, Sumida, Tokyo, Japan).

\section{Light conditions during diurnal LUC activity measurements}

To mimic white light (WL) in LUMINATOR we used mixed LEDs emitting $R$ (590-660nm), $B$ (420500nm) and FR 680-760) light. Moreover, for typical imaging of ff-LUC activity in plants under diurnal "WL" light regime we used a step gradient (ramping) in light intensity and quality to mimic altered light intensity and quality in morning and evening under natural light conditions. The light intensity during 2 hour ramping at start-day and end-day is $33 \mu \mathrm{mole} \mathrm{m}^{-2} \mathrm{~s}^{-1}$ and during the remaining hours of the photoperiod $90 \mu$ mole $\mathrm{m}^{-2} \mathrm{~s}^{-1}$. Photosynthetically active radiation (PAR) intensity was 25 and $80 \mu$ mole $\mathrm{m}^{-2} \mathrm{~s}^{-1}$ respectively.

The ratio $B: R: F R$ light during ramping is $1: 2: 1$ and during the remaining hours of the photoperiod 3:6:1. 
The Red light treatments were at $80 \mu$ mole $\mathrm{m}^{-2} \mathrm{~s}^{-1}$ of pure Red light, the FR light treatment was at $430 \mu$ mole $\mathrm{m}^{-2} \mathrm{~s}^{-1}$ of FR LED light and the Blue light treatment was at $30 \mu$ mole $\mathrm{m}^{-2} \mathrm{~s}^{-1}$ of blue light. The $R>F R$ step gradient light treatment consists of 3 hours $R: F R=8,3$ hours $R: F R=1$ (mild shade), 3 hours R:FR=0.5(shade) and 3 hours R:FR=0.2 (deep shade). PAR intensity was 80-85 $\mu$ mole $\mathrm{m}^{-2} \mathrm{~s}^{-1}$ during all shade conditions. Light quality/intensity was measured the using Flame-T spectroradiometer (Ocean Optics, Duiven, The Netherlands).

\section{Quantifying relative LUC activity in plants}

Luminescence was analysed using Image J software (imagej.nih.gov/ij). Images are imported as stack into Image J, equal square areas covering each individual plant are defined in the ROI manager of Image $J$ and mean grey value is measured for each plant throughout the image stack. Background values were subtracted from the mean grey values to obtain luminescence. Background values were determined by measuring mean grey values of Col-0 WT plants grown under the same experimental conditions as the reporter plants. The data from first day of each experiment was not counted due to acclimation of plant to Luminator cabinet. Relative luminescence $(\mathrm{ZTO}(\mathrm{day} 1)=1)$ was calculated for each individual plant to deal with differences in general luminescence level between individual reporter plants from the same line caused by lens properties, reporter activity and/or differences in shape of individual plants (e.g. petiole length and leaf width).

Quantitative RT-PCR. Relative gene expression of selected gene were quantified by qRT-PCR method. For that WT (Col-0), five phy mutans, and pif4-2 mutant plants were grown for two weeks in White Light and transferred either Red, Far-Red or Blue light treatment. Total RNA was extracted from after treatment and non-treatment sample leaves using InviTrap Spin Plant RNA mini Kit (Berlin, Germany), following manufacturer's instructions. Purified total RNAs were subjected to TURBO DNA-free ${ }^{\mathrm{TM}}$ DNase (Thermo Fisher Scientific Inc., Waltham, Massachusetts) treatment to avoid with contaminated genomic DNA. For reverse transcription the iScrip II mix reagent was used that included $10 \mathrm{mM}$ oligo (dT) primer according to the manufacturer's instruction (Bio-Rad, CA,USA). The primers listed in Table S2 were used for the real time qPCR. As a reference gene we have used AT2G39960 gene (Yang et al., 2018). Reaction were carried out with total RNA isolated from pooled samples from 
three individual plants, with triple biological replicates using SYBR Green PCR Master Mix (BioRad, CA,USA) on the CFX Connect Real Time System machine (Bio-Rad, CA, USA). The Ct method (2- $\Delta \Delta \mathrm{Ct}$ ) was used to analysis the differences in mRNA values (http://www.biorad.com/).

Statistical analyses. All data were subjected to one-way analysis of variance (ANOVA). Comparison of means was analysed for statistical significance with a 2 -sample t-test $(P<0.05$ or $<0.01)$. 


\section{References}

Al-Sady B, Kikis EA, Monte E, Quail PH (2008) Mechanistic duality of transcription factor function in phytochrome signaling. Proc Natl Acad Sci U S A 105: 2232-2237

Bae G, Choi G (2008) Decoding of light signals by plant phytochromes and their interacting proteins. Annu Rev Plant Biol 59: 281-311

Balasubramanian S, Sureshkumar S, Agrawal M, Michael TP, Wessinger C, Maloof JN, Clark R, Warthmann N, Chory J, Weigel D (2006) The PHYTOCHROME C photoreceptor gene mediates natural variation in flowering and growth responses of Arabidopsis thaliana. Nat Genet 38: 711-715

Casal JJ, Candia AN, Sellaro R (2014) Light perception and signalling by phytochrome A. J Exp Bot 65: 2835-2845

Castillon A, Shen H, Huq E (2007) Phytochrome Interacting Factors: central players in phytochrome-mediated light signaling networks. Trends Plant Sci 12: 514-521

Chen J, Sonobe K, Ogawa N, Masuda S, Nagatani A, Kobayashi Y, Ohta H (2013) Inhibition of arabidopsis hypocotyl elongation by jasmonates is enhanced under red light in phytochrome B dependent manner. J Plant Res 126: 161-168

Christians MJ, Gingerich DJ, Hua Z, Lauer TD, Vierstra RD (2012) The light-response BTB1 and BTB2 proteins assemble nuclear ubiquitin ligases that modify phytochrome $B$ and $D$ signaling in Arabidopsis. Plant Physiol 160: 118-134

Clack T, Mathews S, Sharrock RA (1994) The phytochrome apoprotein family in Arabidopsis is encoded by five genes: the sequences and expression of PHYD and PHYE. Plant Mol Biol 25: 413-427

Clack T, Shokry A, Moffet M, Liu P, Faul M, Sharrock RA (2009) Obligate heterodimerization of Arabidopsis phytochromes $\mathrm{C}$ and $\mathrm{E}$ and interaction with the PIF3 basic helix-loophelix transcription factor. Plant Cell 21: 786-799

Fankhauser C (2001) The phytochromes, a family of red/far-red absorbing photoreceptors. J Biol Chem 276: 11453-11456

Foreman J, Johansson H, Hornitschek P, Josse EM, Fankhauser C, Halliday KJ (2011) Light receptor action is critical for maintaining plant biomass at warm ambient temperatures. Plant J 65: 441-452

Franklin KA, Quail PH (2010) Phytochrome functions in Arabidopsis development. J Exp Bot 61: 11-24

Genoud T, Schweizer F, Tscheuschler A, Debrieux D, Casal JJ, Schafer E, Hiltbrunner A, Fankhauser C (2008) FHY1 mediates nuclear import of the light-activated phytochrome A photoreceptor. PLoS Genet 4: e1000143

Hartmann KM (1967) Phytochrome 730 (Pfr), the effector of the "high energy photomorphogenic reaction" in the far-red region. Naturwissenschaften 54: 544

Hennig L, Funk M, Whitelam GC, Schafer E (1999) Functional interaction of cryptochrome 1 and phytochrome D. Plant J 20: 289-294

Hofmann NR (2009) Opposites attract: some phytochromes do not form homodimers. Plant Cell 21: 698

Huq E, Al-Sady B, Hudson M, Kim C, Apel K, Quail PH (2004) Phytochrome-interacting factor 1 is a critical bHLH regulator of chlorophyll biosynthesis. Science 305: 1937-1941 
Jang IC, Henriques R, Seo HS, Nagatani A, Chua NH (2010) Arabidopsis PHYTOCHROME INTERACTING FACTOR proteins promote phytochrome B polyubiquitination by COP1 E3 ligase in the nucleus. Plant Cell 22: 2370-2383

Jung JH, Domijan M, Klose C, Biswas S, Ezer D, Gao M, Khattak AK, Box MS, Charoensawan V, Cortijo S, Kumar M, Grant A, Locke JC, Schafer E, Jaeger KE, Wigge PA (2016) Phytochromes function as thermosensors in Arabidopsis. Science 354: 886-889

Kevei E, Schafer E, Nagy F (2007) Light-regulated nucleo-cytoplasmic partitioning of phytochromes. J Exp Bot 58: 3113-3124

Khanna R, Huq E, Kikis EA, Al-Sady B, Lanzatella C, Quail PH (2004) A novel molecular recognition motif necessary for targeting photoactivated phytochrome signaling to specific basic helix-loop-helix transcription factors. Plant Cell 16: 3033-3044

Khanna R, Shen Y, Marion CM, Tsuchisaka A, Theologis A, Schafer E, Quail PH (2007) The basic helix-loop-helix transcription factor PIF5 acts on ethylene biosynthesis and phytochrome signaling by distinct mechanisms. Plant Cell 19: 3915-3929

Klose C, Viczian A, Kircher S, Schafer E, Nagy F (2015) Molecular mechanisms for mediating light-dependent nucleo/cytoplasmic partitioning of phytochrome photoreceptors. New Phytol 206: 965-971

Legris M, Klose C, Burgie ES, Rojas CC, Neme M, Hiltbrunner A, Wigge PA, Schafer E, Vierstra RD, Casal JJ (2016) Phytochrome B integrates light and temperature signals in Arabidopsis. Science 354: 897-900

Leivar P, Monte E, Oka Y, Liu T, Carle C, Castillon A, Huq E, Quail PH (2008) Multiple phytochrome-interacting bHLH transcription factors repress premature seedling photomorphogenesis in darkness. Curr Biol 18: 1815-1823

Leivar P, Quail PH (2011) PIFs: pivotal components in a cellular signaling hub. Trends Plant Sci 16: $19-28$

Li J, Li G, Wang H, Wang Deng X (2011) Phytochrome Signaling Mechanisms. The Arabidopsis Book / American Society of Plant Biologists 9: e0148

Lorrain S, Allen T, Duek PD, Whitelam GC, Fankhauser C (2008) Phytochrome-mediated inhibition of shade avoidance involves degradation of growth-promoting bHLH transcription factors. Plant J 53: 312-323

Lorrain S, Trevisan M, Pradervand S, Fankhauser C (2009) Phytochrome interacting factors 4 and 5 redundantly limit seedling de-etiolation in continuous far-red light. Plant $\mathrm{J}$ 60: 449-461

Marques SM, Esteves da Silva JC (2009) Firefly bioluminescence: a mechanistic approach of luciferase catalyzed reactions. IUBMB Life 61: 6-17

Millar AJ, Short SR, Chua NH, Kay SA (1992) A novel circadian phenotype based on firefly luciferase expression in transgenic plants. Plant Cell 4: 1075-1087

Monte E, Tepperman JM, Al-Sady B, Kaczorowski KA, Alonso JM, Ecker JR, Li X, Zhang Y, Quail PH (2004) The phytochrome-interacting transcription factor, PIF3, acts early, selectively, and positively in light-induced chloroplast development. Proc Natl Acad Sci U S A 101: 16091-16098

Nagatani A (2004) Light-regulated nuclear localization of phytochromes. Curr Opin Plant Biol 7: 708-711

Nagatani A, Kay SA, Deak M, Chua NH, Furuya M (1991) Rice type I phytochrome regulates hypocotyl elongation in transgenic tobacco seedlings. Proc Natl Acad Sci U S A 88: 5207-5211 
Nagatani A, Reed JW, Chory J (1993) Isolation and Initial Characterization of Arabidopsis Mutants That Are Deficient in Phytochrome A. Plant Physiol 102: 269-277

Nagy F, Schafer E (2002) Phytochromes control photomorphogenesis by differentially regulated, interacting signaling pathways in higher plants. Annu Rev Plant Biol 53: 329355

Ni W, Xu SL, Chalkley RJ, Pham TN, Guan S, Maltby DA, Burlingame AL, Wang ZY, Quail PH (2013) Multisite light-induced phosphorylation of the transcription factor PIF3 is necessary for both its rapid degradation and concomitant negative feedback modulation of photoreceptor phyB levels in Arabidopsis. Plant Cell 25: 2679-2698

Oh E, Zhu JY, Wang ZY (2012) Interaction between BZR1 and PIF4 integrates brassinosteroid and environmental responses. Nat Cell Biol 14: 802-809

Paik I, Yang S, Choi G (2012) Phytochrome regulates translation of mRNA in the cytosol. Proc Natl Acad Sci U S A 109: 1335-1340

Park E, Park J, Kim J, Nagatani A, Lagarias JC, Choi G (2012) Phytochrome B inhibits binding of phytochrome-interacting factors to their target promoters. Plant J 72: 537-546

Parks BM, Quail PH (1993) hy8, a new class of arabidopsis long hypocotyl mutants deficient in functional phytochrome A. Plant Cell 5: 39-48

Possart A, Fleck C, Hiltbrunner A (2014) Shedding (far-red) light on phytochrome mechanisms and responses in land plants. Plant Sci 217-218: 36-46

Proost S, Van Bel M, Vaneechoutte D, Van de Peer Y, Inzé D, Mueller-Roeber B, Vandepoele K (2015) PLAZA 3.0: an access point for plant comparative genomics. Nucleic Acids Research 43: D974-D981

Quail PH, Boylan MT, Parks BM, Short TW, Xu Y, Wagner D (1995) Phytochromes: photosensory perception and signal transduction. Science 268: 675-680

Reed JW, Nagpal P, Poole DS, Furuya M, Chory J (1993) Mutations in the gene for the red/farred light receptor phytochrome $B$ alter cell elongation and physiological responses throughout Arabidopsis development. Plant Cell 5: 147-157

Reed JW, Nagpal P, Poole DS, Furuya M, Chory J (1993) Mutations in the gene for the red/farred light receptor phytochrome $B$ alter cell elongation and physiological responses throughout Arabidopsis development. The Plant Cell 5: 147-157

Sanchez-Lamas M, Lorenzo CD, Cerdan PD (2016) Bottom-up Assembly of the Phytochrome Network. PLoS Genet 12: e1006413

Seaton DD, Toledo-Ortiz G, Ganpudi A, Kubota A, Imaizumi T, Halliday KJ (2018) Dawn and photoperiod sensing by phytochrome A. Proceedings of the National Academy of Sciences 115: 10523

Shen H, Zhu L, Castillon A, Majee M, Downie B, Huq E (2008) Light-induced phosphorylation and degradation of the negative regulator PHYTOCHROME-INTERACTING FACTOR1 from Arabidopsis depend upon its direct physical interactions with photoactivated phytochromes. Plant Cell 20: 1586-1602

Somers DE, Devlin PF, Kay SA (1998) Phytochromes and cryptochromes in the entrainment of the Arabidopsis circadian clock. Science 282: 1488-1490

Sun J, Qi L, Li Y, Chu J, Li C (2012) PIF4-mediated activation of YUCCA8 expression integrates temperature into the auxin pathway in regulating arabidopsis hypocotyl growth. PLoS Genet 8: e1002594

Sun J, Qi L, Li Y, Zhai Q, Li C (2013) PIF4 and PIF5 transcription factors link blue light and auxin to regulate the phototropic response in Arabidopsis. Plant Cell 25: 2102-2114 
Sun Y, Fan X-Y, Cao D-M, He K, Tang W, Zhu J-Y, He J-X, Bai M-Y, Zhu S, Oh E, Patil S, Kim TW, Ji H, Wong WH, Rhee SY, Wang Z-Y (2010) Integration of Brassinosteroid Signal Transduction with the Transcription Network for Plant Growth Regulation in Arabidopsis. Developmental cell 19: 765-777

Takano M, Inagaki N, Xie X, Yuzurihara N, Hihara F, Ishizuka T, Yano M, Nishimura M, Miyao A, Hirochika H, Shinomura $\mathbf{T}$ (2005) Distinct and cooperative functions of phytochromes $A, B$, and $C$ in the control of deetiolation and flowering in rice. Plant Cell 17: 3311-3325

Toth R, Kevei E, Hall A, Millar AJ, Nagy F, Kozma-Bognar L (2001) Circadian clock-regulated expression of phytochrome and cryptochrome genes in Arabidopsis. Plant Physiol 127: 1607-1616

Toufighi K, Brady SM, Austin R, Ly E, Provart NJ (2005) The Botany Array Resource: eNortherns, Expression Angling, and promoter analyses. Plant J 43: 153-163

Van Buskirk EK, Decker PV, Chen M (2012) Photobodies in light signaling. Plant Physiol 158: $52-60$

Whitelam GC, Johnson E, Peng J, Carol P, Anderson ML, Cowl JS, Harberd NP (1993) Phytochrome A null mutants of Arabidopsis display a wild-type phenotype in white light. Plant Cell 5: 757-768

Xu X, Paik I, Zhu L, Huq E (2015) Illuminating Progress in Phytochrome-Mediated Light Signaling Pathways. Trends Plant Sci 20: 641-650

Yang C, Xie F, Jiang Y, Li Z, Huang X, Li L (2018) Phytochrome A Negatively Regulates the Shade Avoidance Response by Increasing Auxin/Indole Acidic Acid Protein Stability. Developmental Cell 44: 29-41.e24

Yanovsky MJ, Casal JJ, Luppi JP (1997) The VLF loci, polymorphic between ecotypes Landsberg erecta and Columbia, dissect two branches of phytochrome A signal transduction that correspond to very-low-fluence and high-irradiance responses. Plant J 12: 659-667

Zhang X, Henriques R, Lin SS, Niu QW, Chua NH (2006) Agrobacterium-mediated transformation of Arabidopsis thaliana using the floral dip method. Nat Protoc 1: 641646 


\section{Supplements.}

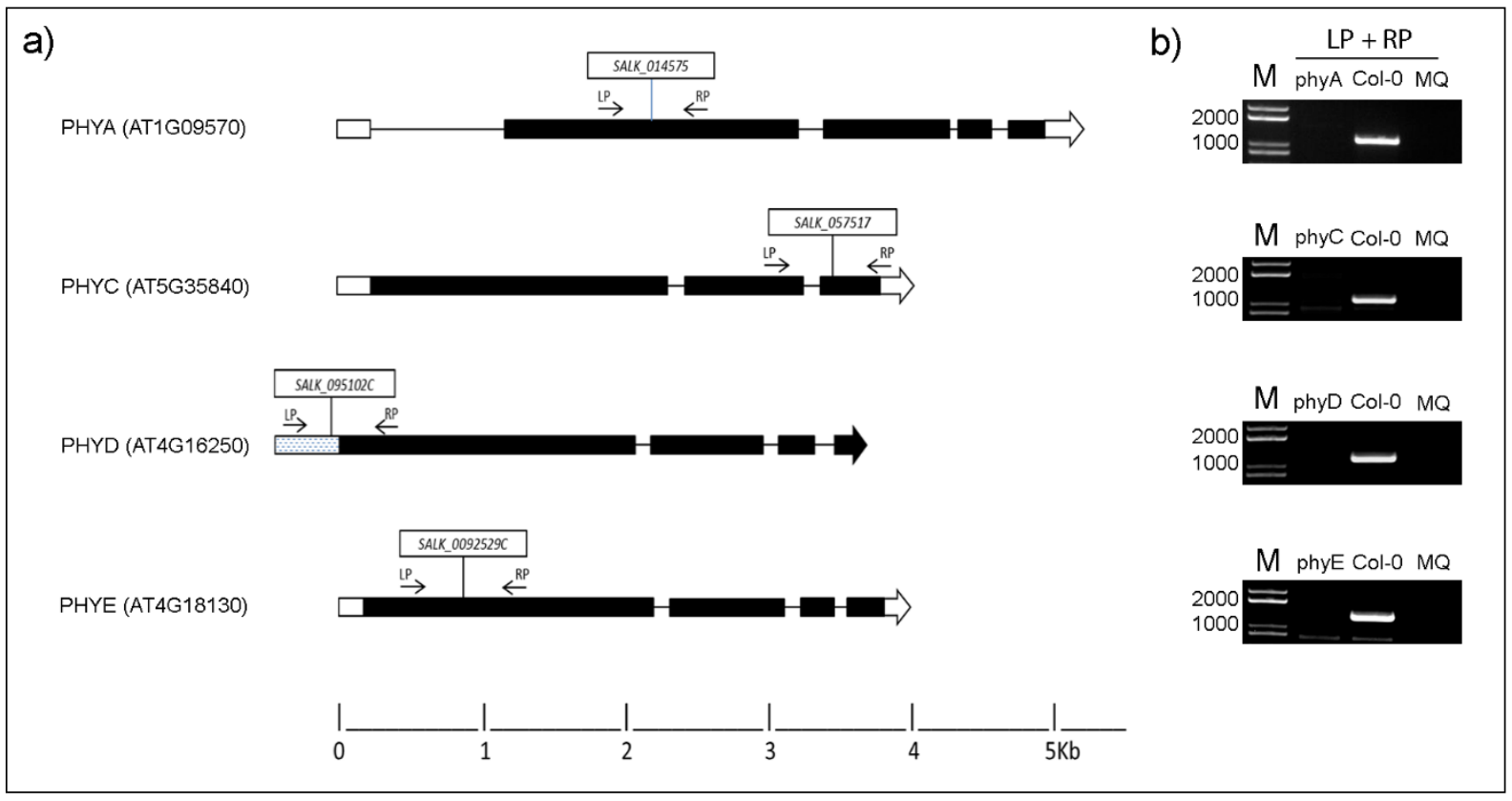

Figure S-1. Genotyping of WT and phy mutants by Alternative PCR and Different light responses. a) Structure of Salk T-DNA insertion for PHY genes. b) Genotyping by gene specific PCR primers (Table S2) in 1\% agarose gel. 


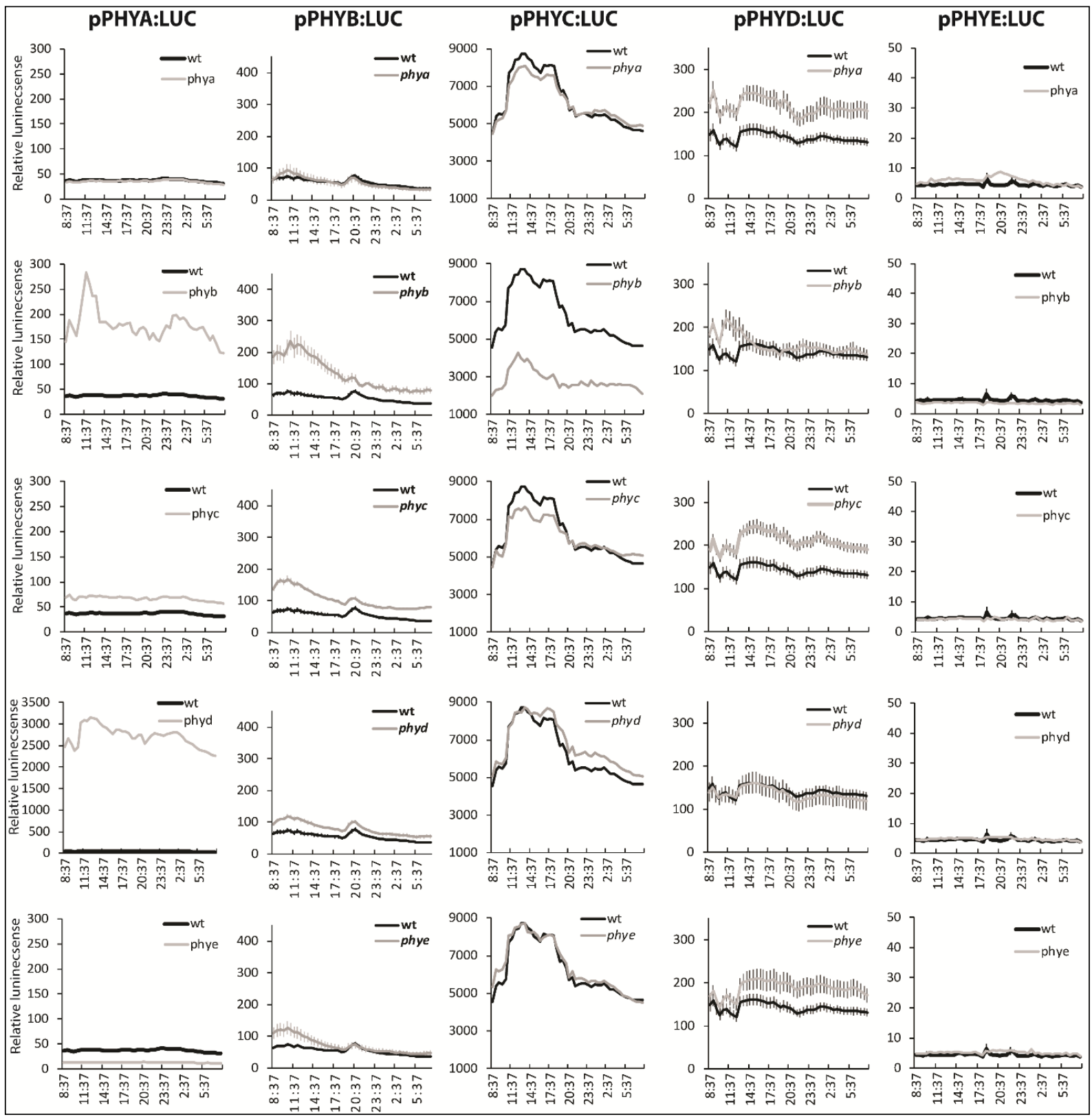

Figure S2. A diurnal pPHY:LUC activity in WT and phy mutant plants under mixed R+B+FR light. Plants were pre-grown for 25 days in growth cabinets under fluorescent WL and sprayed with substrate luciferin (1 mM) one day before placing in LUMINATOR with diurnal R+B+FR LED light regime. After adaptation for one day in LUMINATOR, LUC activity images were obtained every half hour (7 min. exposure) for a full diurnal cycle under mixed LED light. The relative LUC activity was quantified in Image J and corrected for background signal. Number of replicate plants for each reporter line: $\mathrm{N}=6$. Error bars represent mean $\pm \mathrm{SE}$. 


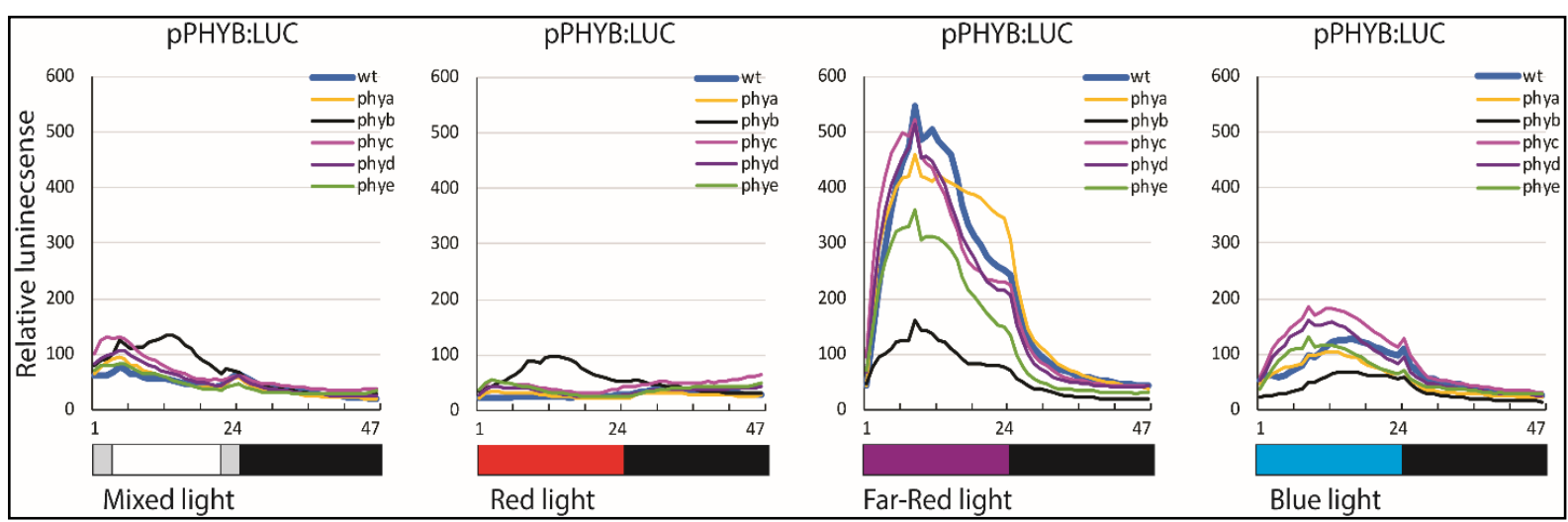

Figure S3. pPHYB-LUC activity in 14 day old seedings in WT and phy mutants under mixed, R, FR and

B LED. Seeds of PPHYB-LUC reporter in WT and the five single PHY mutant backgrounds were stratified and germinated in growth cabinets under diurnal fluorescent WL (12L/12D). At 14 days after germination seedlings were sprayed with substrate luciferin $(1 \mathrm{mM})$ and one day later placed in LUMINATOR for adjustment under diurnal $R+B+F R$ for one day. Subsequently plants were exposed to light regimes 12 mixed/12D, 12R/12D, followed by $12 \mathrm{FR} / \mathrm{D}$ and finally $12 \mathrm{~B} / 12 \mathrm{D}$. Luciferin $(1 \mathrm{mM})$ solution was sprayed once per day. LUC activity images were obtained every half hour (7 min. exposure) for each full diurnal cycle. The relative LUC activity was quantified in Image J and corrected for background signal. Number of replicate seedlings for each reporter line: $\mathrm{N}=6$. Error bars represent mean $\pm \mathrm{SE}$. 
A)
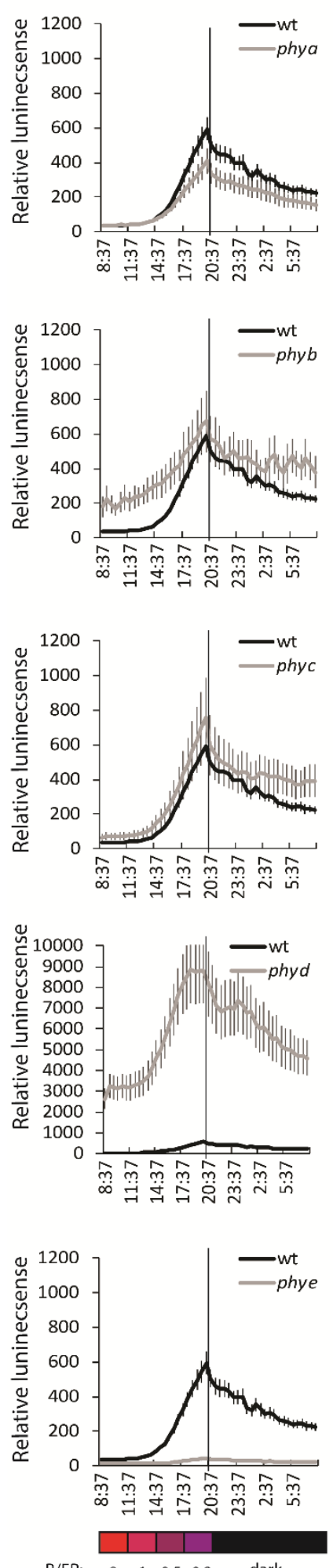

PPHYA:LUC
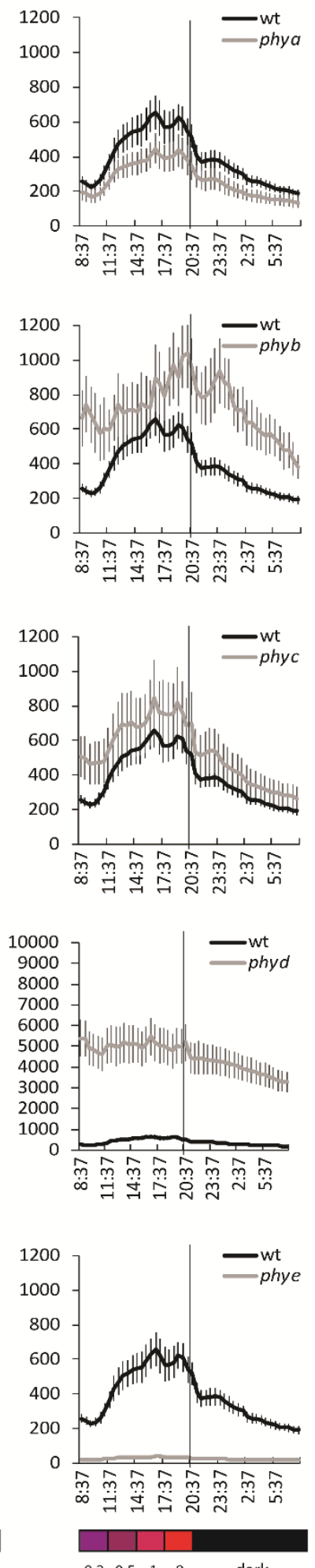

B)

PPHYB:LUC
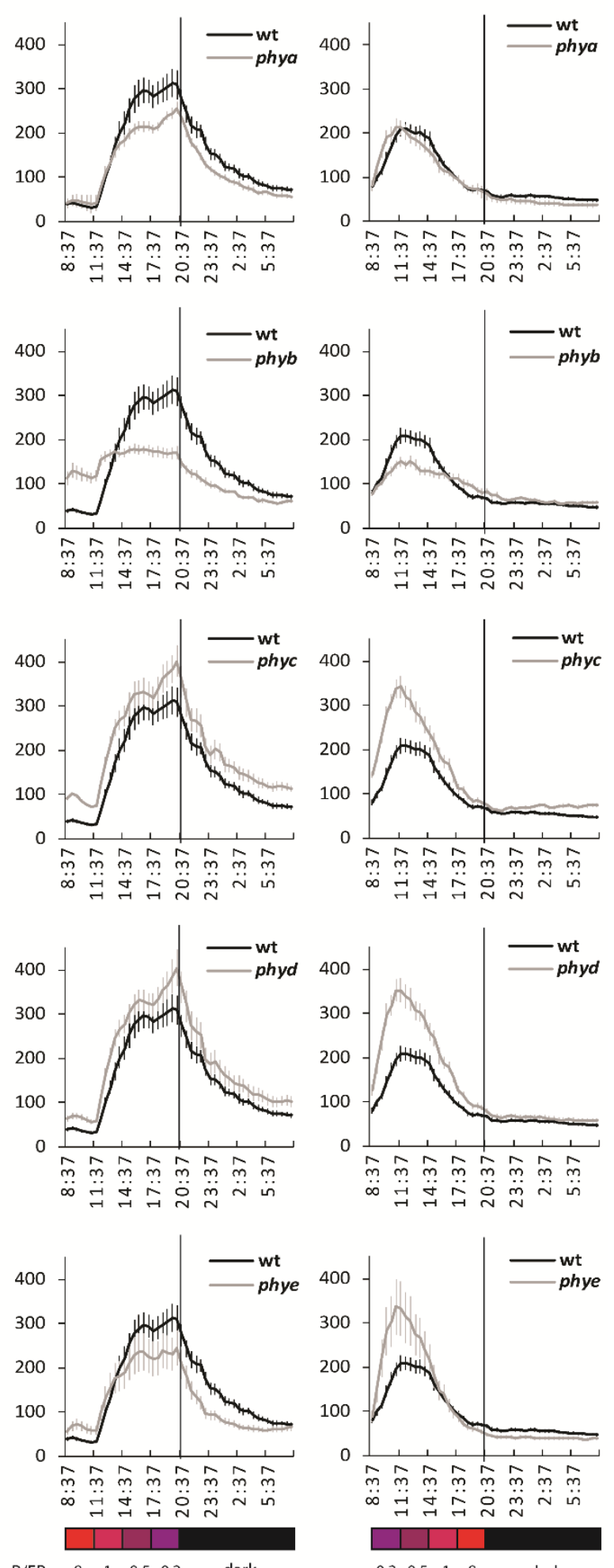

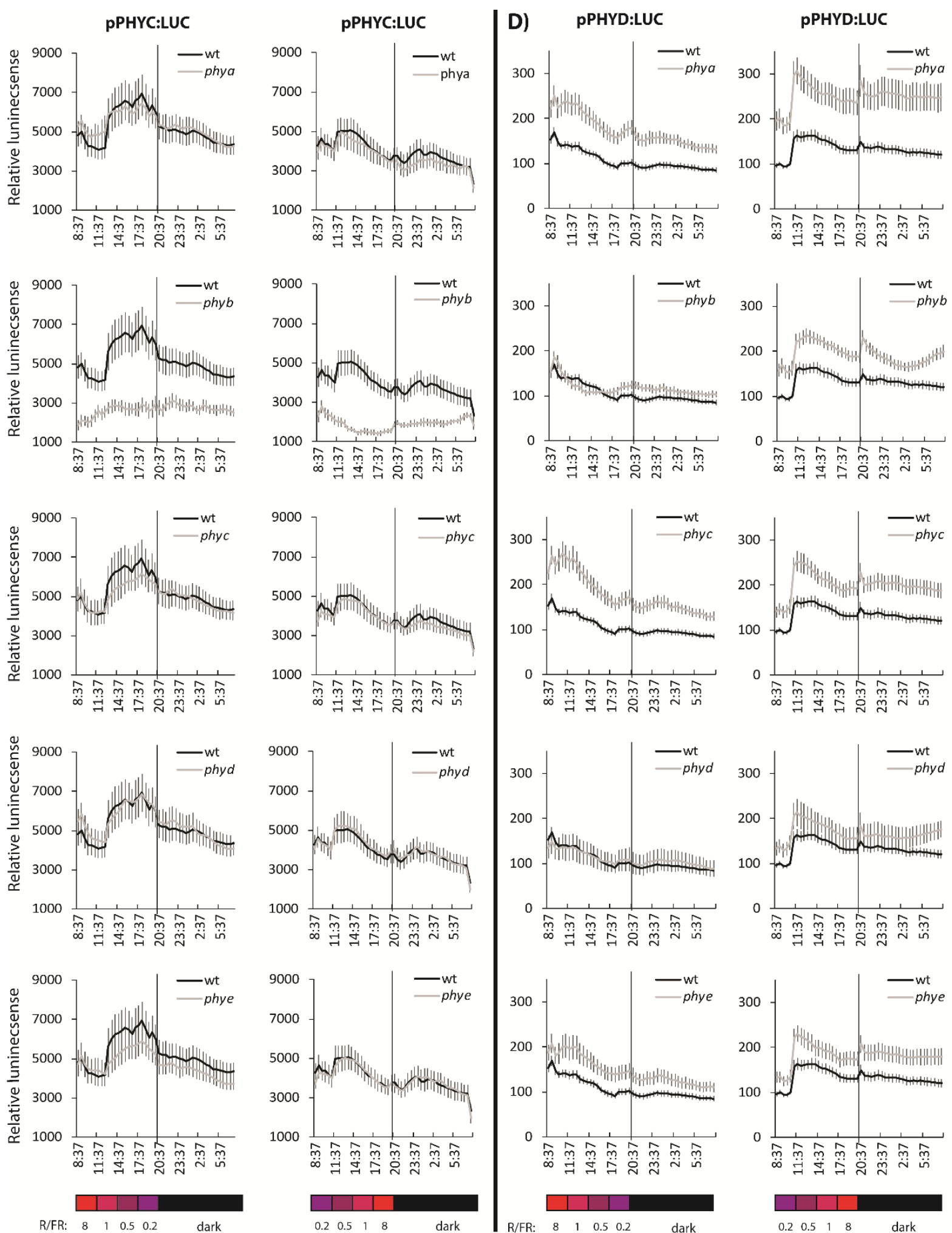

Figure S4. pPHY:LUC activity in phy mutant compared WT rosette plants in response to changing R:FR

ratios. Seeds of all WT and phy mutant PPHY:LUC reporter lines were stratified and germinated in growth cabinets under diurnal fluorescent WL (12L/12D). At 25 days after germination plants were sprayed with 
substrate luciferin (1 $\mathrm{mM}$ ) and one day later placed in LUMINATOR for adjustment under diurnal mixed $R+B+F R$ for one day. Subsequently seedlings were exposed to R light with increasing levels of FR (in blocks of 3 hours), resulting in R:FR ratio's of $8,1,0.5$ and 0.2 . After the following night plants were exposed to the reverse light regime. Luciferin (1 $\mathrm{mM}$ ) solution was sprayed once per day. LUC activity images were obtained every half hour (7 min. exposure) for each full diurnal cycle. The relative LUC activity was quantified in Image J and corrected for background signal. Number of replicate seedlings for each reporter line: $N=6$. Error bars represent mean $\pm S E$.

A: pPHYA:LUC activity in WT and the five phy mutant backgrounds. Note that for the phyD mutant background the scale of relative LUC activity is different. B: pPHYB:LUC activity in WT and the five phy mutant backgrounds. C: pPHYC:LUC activity in WT and the five phy mutant backgrounds. D: PPHYD:LUC activity in WT and the five phy mutant backgrounds. pPHYE:LUC activity in WT and the five phy mutant backgrounds showed barely above background (data not showed). 


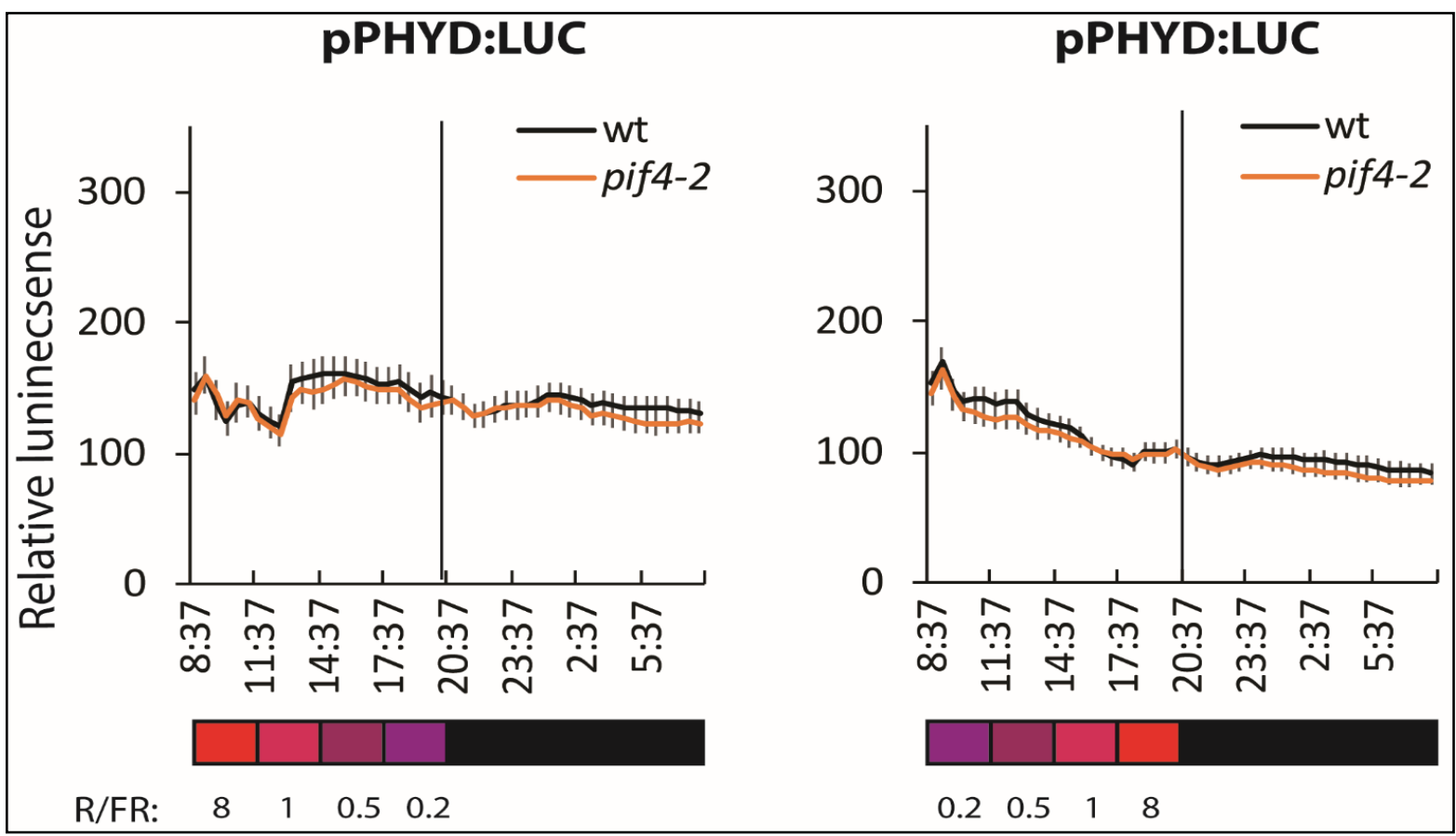

Figure S5. PIF4 is not involved pPHYD:LUC activity. A 25 days after germination pPHYD:LUC reporter in WT and pif4-2 mutant backgrounds were exposed to R light with increasing levels of FR (in blocks of 3 hours), resulting in R:FR ratio's of $8,1,0.5$ and 0.2 . After the following night plants were exposed to the reverse light regime. LUC activity images were obtained every half hour ( $7 \mathrm{~min}$. exposure) for each full diurnal cycle. The relative LUC activity was quantified in Image J. Background value subtracted from average of observed value (OB). Number of replicate seedlings for each reporter line: $\mathrm{N}=11$. Error bars represent mean $\pm \mathrm{SE}$. In conclusion, PIF4 does not regulates PHYD expression although it was found G-box motif in the promoter site of PHYD. 


\begin{tabular}{|c|c|c|c|}
\hline $\begin{array}{l}\text { LUC reporter lines, } \\
\text { constructs }\end{array}$ & Description & $\begin{array}{l}\text { Plasmid } \\
\text { notes }\end{array}$ & Reference \\
\hline pPHYA:LUCWT & pPHYA:LUC reporter in Col-0 & $H y g^{\mathrm{R}} / \mathrm{pPCVH}$ & this study \\
\hline pPHYA:LUC phyA & pPHYA:LUC reporter crossed to phyA mutant & $H_{y g}{ }^{R} / p P C V H$ & this study \\
\hline pPHYA:LUC ${ }^{\text {phyB-9 }}$ & pPHYA:LUC reporter crossed to phyB-9 mutant & $H y g^{R} / p P C V H$ & this study \\
\hline pPHYA:LUC ${ }^{\text {phyc }}$ & pPHYA:LUC reporter crossed to phyC mutant & $H y g^{\mathrm{R}} / \mathrm{pPCVH}$ & this study \\
\hline pPHYA:LUC ${ }^{p h D}$ & pPHYA:LUC reporter crossed to phyD mutant & $H y g^{R} / p P C V H$ & this study \\
\hline pPHYA:LUC ${ }^{\text {phyE }}$ & pPHYA:LUC reporter crossed to phyE mutant & $H y g^{R} / p P C V H$ & this study \\
\hline pPHYB:LUC ${ }^{W T}$ & pPHYB:LUC reporter in Col-0 & $H y g^{\mathrm{R}} / \mathrm{pPCVH}$ & this study \\
\hline pPHYB:LUC ${ }^{\text {phyA }}$ & pPHYB:LUC reporter crossed to phyA mutant & $H y g^{\mathrm{R}} / \mathrm{pPCVH}$ & this study \\
\hline pPHYB:LUC ${ }^{\text {phyB-9 }}$ & pPHYB:LUC reporter crossed to phyB-9 mutant & $H y g^{R} / p P C V H$ & this study \\
\hline pPHYB:LUC ${ }^{\text {phyc }}$ & pPHYB:LUC reporter crossed to $p h y C$ mutant & $H y g^{\mathrm{R}} / \mathrm{pPCVH}$ & this study \\
\hline pPHYB:LUC ${ }^{p h D}$ & pPHYB:LUC reporter crossed to phyD mutant & $H y g^{R} / p P C V H$ & this study \\
\hline pPHYB:LUC ${ }^{\text {phyE }}$ & pPHYB:LUC reporter crossed to phyE mutant & $H y g^{\mathrm{R}} / \mathrm{pPCVH}$ & this study \\
\hline pPHYC:LUCWT & pPHYC:LUC reporter in Col-0 & $H y g^{\mathrm{R}} / \mathrm{pPCVH}$ & this study \\
\hline pPHYC:LUC ${ }^{\text {phyA }}$ & pPHYC:LUC reporter crossed to phyA mutant & $H_{y g}{ }^{\mathrm{R}} / \mathrm{pPCVH}$ & this study \\
\hline pPHYC:LUC ${ }^{\text {phyB-9 }}$ & pPHYC:LUC reporter crossed to phyB-9 mutant & $H_{y g}{ }^{\mathrm{R}} / \mathrm{pPCVH}$ & this study \\
\hline pPHYC:LUC ${ }^{\text {phyc }}$ & pPHYC:LUC reporter crossed to phyC mutant & $H y g^{R} / p P C V H$ & this study \\
\hline pPHYC:LUC ${ }^{p h D}$ & pPHYC:LUC reporter crossed to phyD mutant & $H y g^{\mathrm{R}} / \mathrm{pPCVH}$ & this study \\
\hline pPHYC:LUC $C^{\text {phyE }}$ & pPHYC:LUC reporter crossed to phyE mutant & $H y g^{R} / p P C V H$ & this study \\
\hline pPHYD:LUCWT & pPHYD:LUC reporter in Col-0 & $H y g^{\mathrm{R}} / \mathrm{pPCVH}$ & this study \\
\hline pPHYD:LUC ${ }^{\text {phyA }}$ & pPHYD:LUC reporter crossed to phyA mutant & $H_{y g}{ }^{R} / p P C V H$ & this study \\
\hline pPHYD:LUC ${ }^{\text {phyB-9 }}$ & pPHYD:LUC reporter crossed to phyB-9 mutant & $H y g^{\mathrm{R}} / \mathrm{pPCVH}$ & this study \\
\hline pPHYD:LUC ${ }^{\text {phyc }}$ & pPHYD:LUC reporter crossed to $p h y C$ mutant & $H y g^{R} / p P C V H$ & this study \\
\hline pPHYD:LUC ${ }^{p h D}$ & pPHYD:LUC reporter crossed to phyD mutant & $H y g^{R} / p P C V H$ & this study \\
\hline pPHYD:LUC ${ }^{\text {phyE }}$ & pPHYD:LUC reporter crossed to phyE mutant & $H y g^{\mathrm{R}} / \mathrm{pPCVH}$ & this study \\
\hline pPHYE:LUCWT & pPHYE:LUC reporter in Col-0 & $H y g^{\mathrm{R}} / \mathrm{pPCVH}$ & this study \\
\hline
\end{tabular}




\begin{tabular}{|c|c|c|c|}
\hline pPHYE:LUC ${ }^{\text {phyA }}$ & pPHYE:LUC reporter crossed to phyA mutant & $H y g^{\mathrm{R}} / \mathrm{pPCVH}$ & this study \\
\hline pPHYE:LUC ${ }^{\text {phyB-9 }}$ & pPHYE:LUC reporter crossed to phyB-9 mutant & $H y g^{R} / p P C V H$ & this study \\
\hline pPHYE:LUC ${ }^{\text {phyc }}$ & pPHYE:LUC reporter crossed to $p h y C$ mutant & $H y g^{\mathrm{R}} / \mathrm{pPCVH}$ & this study \\
\hline pPHYE:LUC & pPHYE:LUC reporter crossed to phyD mutant & $H y g^{R} / p P C V H$ & this study \\
\hline pPHYE:LUC ${ }^{\text {phyE }}$ & pPHYE:LUC reporter crossed to phyE mutant & $H y g^{R} / p P C V H$ & this study \\
\hline pPHYB:LUC & pPHYB:LUC reporter crossed to pif4-2 mutant & $H y g^{\mathrm{R}} / \mathrm{pPCVH}$ & this study \\
\hline pPHYD:LUC & pPHYD:LUC reporter crossed to pif4-2 mutant & $H y g^{R} / p P C V H$ & this study \\
\hline 35S:PHYB & Expression vector & $S p^{R} / p K G W$ & this study \\
\hline 35S:PIF4 & Expression vector & $S p^{R} / p K G W$ & this study \\
\hline 35S:BZR1 & Expression vector & $S p^{R} / p K G W$ & this study \\
\hline
\end{tabular}

Table-S1. List of reporter lines and expression constructs were used in study. The PPHY:LUC reporter was created in Col-0 and its crossed with different mutant background lines. 


\begin{tabular}{|c|c|c|c|}
\hline № & Sequence (5'-3') & Target & Description \\
\hline & ccagtcagctcagcaattttc -LB & PHYA & Screening of mutant \\
\hline \multirow[t]{2}{*}{1} & aatgcaaaacatgctagggtg $-\mathrm{RB}$ & (AT1G09570) & \\
\hline & ttaggcttacgtagcttcccc -LB & PHYC & Screening of mutant \\
\hline \multirow[t]{2}{*}{2} & gatggagctgagcatagaacg $-\mathrm{RB}$ & (AT5G35840) & \\
\hline & gctttttacacgaatcttgcg -LB & PHYD & Screening of mutant \\
\hline \multirow[t]{2}{*}{3} & agtctcgcgtcgacagtgtac $-\mathrm{RB}$ & (AT4G16250) & \\
\hline & aaagaggcggtctagttcagc -LB & PHYE & Screening of mutant \\
\hline \multirow[t]{2}{*}{4} & tatcagtggttaaacccgtcg -RB & (AT4G18130) & \\
\hline & acctcctcaagtcatggttaagcctaagcc -LB & PIF4 & Screening of mutant \\
\hline 5 & tccaaacgagaaccgtcggt -RB & (AT2G43010) & \\
\hline 6 & attttgccgatttcggaac -LB & SALK T-DNA & Screening of mutant \\
\hline \multirow[t]{2}{*}{7} & tagcatctgaatttcataaccaatctcgatacac -LB & SAIL T-DNA & Screening of mutant \\
\hline & agagatacgccctggttcct $-\mathrm{F}$ & & \\
\hline \multirow[t]{2}{*}{8} & ctgttgagcaattcacgttca -R & LUC & qPCR \\
\hline & tccactgggtattgtgtcgc $-\mathrm{F}$ & & \\
\hline 9 & agctatctcctgcaggtgga $-\mathrm{R}$ & PHYA & qPCR \\
\hline \multirow[t]{3}{*}{10} & cgttgggtgttgctcctagt $-\mathrm{F}$ & & \\
\hline & gataccccgcatcgcctaaa $-\mathrm{R}$ & PHYB & qPCR \\
\hline & tccgccatgaagtgaaggac $-F$ & & \\
\hline \multirow[t]{2}{*}{12} & ccgaattcgctgcaatccag $-\mathrm{R}$ & PHYC & qPCR \\
\hline & cgattcctccgtaccagagc $-\mathrm{F}$ & & \\
\hline \multirow[t]{2}{*}{13} & tttcccgcgcattttcactg $-\mathrm{R}$ & PHYD & qPCR \\
\hline & attgaaaccgcaactgcacc $-\mathrm{F}$ & & \\
\hline \multirow[t]{3}{*}{14} & tcatcggcaagtgacttccc $-\mathrm{R}$ & PHYE & qPCR \\
\hline & ccatcgacagtgctgatcca- $\mathrm{F}$ & & Housekeeping for \\
\hline & & & qPCR \\
\hline 15 & ccattgggtgacacttttggt-R & AT2G39960 & \\
\hline
\end{tabular}

Table-S2. Primers were used for this study. 


\section{CHAPTER-3}

Feedback at the PIF4 locus: PIF4 is a negative regulator of its own expression

Umidjon Shapulatov

Piet Laurens

Viola Willemsen

Mark van Hoogdalem

Christa Testerink

Alexander van der Krol

In preparation for publication 


\begin{abstract}
Growth and specific growth responses like shade avoidance or thermomorphogenesis induced plant elongation responses are mediated by transcription factors PIF4 and BZR1. The brassinosteroid (BR) signalling activated transcription factor BZR1 is a positive factor for PIF4 gene expression and together PIF4 and BZR1 regulate genes required for elongation. Moreover, PIF4 protein activates BR biosynthesis and BR signalling, which leads to more nuclear active BZR1 to stimulate PIF4 gene expression even further. It is proposed that the potential runaway activity of this positive feedback regulation is limited by factors that act at the post-translational level on PIF4. Here we identify the PIF4 protein itself as a dominant factor to limit PIF4 gene transcription. Both in transient expression assays and in PIF4-LUC reporter plants, PIF4-LUC reporter activity is suppressed by PIF4 overexpression. However, because of the complicated multiple feedback regulation on PIF4 it remains difficult to predict PIF4 activity in mutant background pif4-2 or bzr1-1D. n WT and mutants plants. Combined, these studies uncover a novel direct negative feedback interaction on PIF4 gene activity, which counterbalance the positive feedback interaction of BZR1 on PIF4 gene activity and demonstrate a remarkable buffering capacity for PIF4 gene activity under different conditions.
\end{abstract}

Key words: PIF4, BZR1, Feedback regulation, 


\section{Introduction}

Regulation of plant cell elongation is an important factor in how plants deal with varying environmental signals such as light and temperature. For instance, light quality conditions in canopy shade may trigger stem cell elongation, which is important for plant survival in order to outgrow neighbouring plants that compete for direct sunlight. Similarly, temperature has a strong effect on plant cell elongation, as demonstrated by the reduced elongation of plants under a regime of alternative day/night temperatures (Thingnaes et al., 2003; Bours et al., 2012; Cagnola et al., 2012; Bours et al., 2013; Bours et al., 2015). Elongation is also an important response for plants under high ambient temperature (warmth), even when this temperature is still below the range that may induce heat stress. The warmth-induced elongation response is named thermomorphogenesis and for Arabidopsis results in a more open plant architecture, which allows for better cooling of leaves (Jung et al., 2016; Delker et al., 2017). The molecular components involved in normal growth, shade avoidance induced elongation or warmth induced elongation are mostly the same, but activity of individual components may be modulated by different environmental conditions (Box et al., 2015; Jung et al., 2016; Ma et al., 2016). Key players in growth responses are the Phytochrome Interacting Factor (PIF) transcription factors of which PIF4 has been studied most intensively (Huq and Quail, 2002; Sun et al., 2012; Choi and Oh, 2016).

The role of PIF4 in the plant elongation response is very complex as PIF4 transcription, PIF4 protein-stability and PIF4 protein-activity are affected by numerous factors whose activities may be regulated by the clock, by light or by temperature. The brassinosteroid (BR)activated transcription factor BZR1 is a key regulator of PIF4 transcription, acting in a positive feedback loop in which upregulation of PIF4 gene expression by BZR1 results in biosynthesis of BR and BR signalling, which may lead to more active nuclear BZR1 (Ibañez et al., 2018). The role of PIF4 in BR biosynthesis was recently elucidated: the BZR1 homo-dimer binds to BRREand G-box elements in the promoters of BR biosynthetic genes and inhibits their expression. The BZR1 and PIF4 proteins can physically interact to form a BZR1/PIF4 hetero-dimer (Oh et al., 2012), and this heterodimer is not active on BR biosynthetic genes (Martínez et al., 2018). Thus, at elevated PIF4 levels, formation of the BZR1/PIF4 heterodimer competes with the formation of the repressor BZR1 homo-dimer, resulting in de-repression of BR biosynthesis 
(Martínez et al., 2018). The increased BR signaling subsequently blocks activity of the kinase BIN2, a repressor of BZR1 (He et al., 2002), resulting in more nuclear BZR1 activity. In addition, because BIN2 also phosphorylates PIF4 to target PIF4 for destruction (Bernardo-Garcia et al., 2014), the reduced BIN2 activity results in stabilization of PIF4 protein.

Supposedly, the positive feedback on PIF4 transcription and PIF4 protein stability is kept under control by the other factors that act on PIF4 at the post-translational level. Indeed, multiple kinases (PPKs, CK2, BIN2, and phytochrome itself) and multiple families of ubiquitin ligases (SCFEBF 1/2, CUL3LRB, CUL3BOP, and CUL4COP1-SPA) regulate PIF4 protein stability (Pham et al., 2017). For instance, light activated phytochromes promote the turnover of PIFs through phosphorylation, ubiquitination, and proteasome-mediated degradation (Park et al., 2012; Xu et al., 2015). The transcriptional activity of PIF4 protein is limited by ELF3, which links PIF4 activity to the circadian clock (Box et al., 2015; Raschke et al., 2015; Zhu et al., 2016). PIF4 transcriptional activity is also blocked by light activated CRY1, linking PIF4 activity to blue light signaling (Ma et al., 2016; Pedmale et al., 2016) and PIF4 is sequestered by Della proteins, linking PIF4 activity to gibberellin (GA) signaling (Li et al., 2016). The transcriptional activity of PIF4 is also modulated by competition for binding to the G/E-box in PIF4-target promoters by HY5 (Toledo-Ortiz et al., 2014; Box et al., 2015; Gangappa and Kumar, 2017). The molecular and genetic control of plant thermomorphogenesis and the role of PIF4 has recently been reviewed (Choi and Oh, 2016; Quint et al., 2016).

The G-box and E-box motifs are enriched in PIF4 target genes and the PIF4 promoter also contains a G-box and E-box (Pfeiffer et al., 2014; Ibañez et al., 2018). However, the role of PIF4 in transcriptional regulation of the PIF4 gene has not specifically been investigated. Here we studied the role of PIF4 on PIF4 promoter activity and show that PIF4 is a negative factor for its own gene transcription. This is demonstrated by the reduced endogenous PIF4 expression upon ectopic overexpression of PIF4 in stable transformed Arabidopsis plants. Moreover, PIF4 overexpression suppresses pPIF4:LUC reporter activity in transient expression assays in N.benthamiana leaves, even when co-expressed with BZR1. In addition, we analysed the activity of a pPIF4:LUC reporter construct in stable transformed Arabidopsis WT and mutant plants. Results show that compared to WT the pPIF4:LUC activity is strongly decreased in a PIF4OE background. Surprisingly, pPIF4:LUC is also mildly decreased in a pif4-2 mutant 
background and in a gain of function mutant in which BZR1 is constitutively active (bzr1-1D). pPIF4:LUC activity in WT and mutants was also monitored under warmth and different light conditions, indicating that PIF4 transcription is buffered against changes in both BZR1 and PIF4 activity. Combined, we have identified a direct negative feedback component in the regulation of PIF4, which help counterbalance the positive feedback by BZR1. But we also show that is remains difficult to integrate our extensive knowledge on dynamic PIF4 regulation to predict PIF4 activity under different conditions without computer modelling.

\section{Materials and Methods}

\section{Plant material and growth conditions.}

The Arabidopsis thaliana (L.) mutant lines were obtained from the Nottingham Arabidopsis Stock Centre (NASC). Mutant plants used in these studies were all in Col-0 genetic background and are listed in Table 1. The different reporter lines used here are listed in Table 2. Double mutant and transgenic lines were created in this study.

For the Arabidopsis plant experiments seeds of the different genotypes were first incubated for five days at $4^{\circ} \mathrm{C}$ in darkness. After cold treatment the imbibed seeds were sown onto rock wool blocks. The Arabidopsis plants were then grown in at $12 \mathrm{~L} 22^{\circ} \mathrm{C} / 12 \mathrm{D} 12^{\circ} \mathrm{C}$ on Hoaglandnutrient solution. Seven days old reporter seedlings were used to measuring LUC activity.

Table 1. List of mutants and transgenic lines were used in study.

\begin{tabular}{lll}
\hline Mutants & Description & Ref. \\
\hline pif4-2 & T-DNA insertion mutant (SAIL_1288_E07) & NASC stock \\
bzr1-1D & Gain of function point mutant & NASC stock \\
PIF4OEWT & 35S:PIF4 in Col-0 & this Study \\
PIF4OE ${ }^{\text {bzr1-1D }}$ & 35S:PIF4 in bzr1-1D & this study \\
\hline
\end{tabular}

The pif4-2 mutant line was confirmed to be homozygous by PCR on genomic DNA using T-DNA insertion (SAIL LB-2 primer) and gene-specific primer sets. For screening of bzr1-1D 
homozygous lines, a 306 bp BZR1 DNA fragment was amplified from genomic DNA using the BZR1 primer set. The resulting DNA fragment from bzr1-1D unable to digestion with Hpall restriction enzyme ( $\left.C^{\wedge} C G G\right)$, while the fragment amplified from WT DNA is digested into a 119 bp and $189 \mathrm{bp}$ fragment.

Transformants with PIF4 overexpression (PIF4OE) were selected by the red seed coat marker which is included in the PIF4OE construct. For fair comparison of LUC activity in WT and mutant lines, the LUC reporters were crossed into mutant background (no position effect between genotypes).

Table 2. List of reporter lines were used in study. The pPIF4:LUC reporter was created in Col-0 and its crossed with different background lines.

\begin{tabular}{|c|c|c|}
\hline Reporter lines & Description & Ref. \\
\hline pPIF4:LUCWT & pPIF4:LUC reporter in Col-0 & this study \\
\hline pPIF4:LUC pif4-2 $^{\text {p }}$ & pPIF4:LUC reporter crossed pif4-2 & this study \\
\hline pPIF4:LUC bzr1-1D & pPIF4:LUC reporter crossed bzr1-1D & this study \\
\hline pPIF4:LUC PIF4OE & pPIF4:LUC reporter crossed 35S:PIF4 in Col-0 & this study \\
\hline
\end{tabular}

\section{Cloning of expression constructs and LUC reporter constructs.}

The LUC reporters, PIF4 and BZR1 overexpression constructs were made using standard cloning techniques. For the ectopic expression of PIF4 the PIF4 CDNA was amplified and cloned into a binary expression vector under control of the CaMV 35S promoter. Cloning details will be given on request. For construction of the pPIF4:LUC reporter constructs the $2487 \mathrm{bp}$ intergenic region upstream of the PIF4 (At2g43010) start codon was amplified by PCR (primers listed in Table S1) using Q $5^{\circledR}$ High-Fidelity DNA polymerase (New England Biolabs, Ipswich, MA, USA) and cloned by TOPO ${ }^{\circledR}$ Cloning reaction (Invitrogen, Carlsbad, CA, USA) into the pENTRTM TOPO ${ }^{\circledR}$ entry vector. To generate pPIF4::LUC expression constructs the entry vectors containing the PIF4 promoter sequences were recombined into the pGREENGW:LUC68 destination vector by LR recombination using Gateway ${ }^{\circledR}$ LR Clonase ${ }^{\circledR}$ II enzyme mix (Invitrogen, Carlsbad, CA, USA). The pPIF4:LUC expression constructs were transformed into Agrobacterium tumefaciens (AGLO). 


\section{Transformation and selection of homozygous lines.}

Agrobacterium tumefaciens was used for plant transformation using the floral dip method as described (Zhang et al., 2006). PIF4 OE transgenic T0 seeds were identified by DsRed pigmentation of the seed coat. It should be notes that 12 out of 15 TO PIF4OE plants did not produce seeds. Therefore, seeds harvested from three T1 PIF4OE plants may not be representative of PIF4 overexpression. Homozygous PIF4OE plants were selected from the T2 generation.

Same story for bzr1-1D transformed with 35S:PIF4 by floral dipping. Over 14 positive T0 seeds were grown from this transformation. From these $14 \mathrm{~T} 1$ plants were grown of which most plants did not set seed, again indicating that PIF4OE has severe effects on seed set. Only from two plants T2 seeds could be harvested from which a homozygous mutant bzr1-1D PIF4OE line was developed.

\section{Gene Expression Analysis by Quantitative Real-Time RT-PCR (qPCR).}

For gene expression analysis total RNA was isolated from pooled seedlings or pooled rosette leaves using the InviTrap ${ }^{\circledR}$ Spin Plant RNA Kit and treated with Ambion ${ }^{\circledR}$ TURBO DNA-free Kit according to the manufacturer's instructions. cDNA synthesis was performed using Super Script III RT KIT (Invitrogen) from purified total RNA. qPCR was carried out using iQ SYBR Green Super mix (Bio-Rad) and gene specific primers. The AT1G13320 gene was used as a reference gene for normalization of relative gene expression levels. The primers used are listed in Table S1.

\section{Imaging and quantification of in planta Luciferase activity}

For the imaging of LUC-reporter activity in stable transformed Arabidopsis plants, the plants were pre-sprayed with $1 \mathrm{mM} \mathrm{D}$ :Luciferin (Duchefa, Haarlem, NL) 24 hour prior to imaging to inactivate accumulated luciferase protein. Spraying with D:LUCiferin was repeated one hour before imaging. Imaging was with an $\left(-80^{\circ} \mathrm{C}\right)$ air-cooled $\mathrm{CCD}$ Pixis $1024 \mathrm{~B}$ camera system (Princeton Instruments, Massachusetts, USA) equipped with a $35 \mathrm{~mm}, 1: 1.4$ Nikkon SLR camera lens (Nikon, Tokyo, Japan) fitted with a DT Green filter ring (Image Optics Components Ltd, Orsay, France) to block chlorophyll fluorescence. Exposure time for the LUC activity 
measurements is as indicated. For each reporter line the average LUC activity is given from at least 18 individual seedlings or 8 rosette plants. For the imaging of LUC activity in transient assays, agro-infiltrated leaves were sprayed with $1 \mathrm{mM}$ D:LUCiferin at $24 \mathrm{hr}$ and $1 \mathrm{hr}$ before imaging (7 minutes exposure time). Leaves were harvested at 4 day post agro-infiltration. Relative luminescence from LUC activity was quantified in ImageJ (Bethesda, Maryland, USA), using background subtraction. For each treatment in the transient assays, the average LUC activity in leaves from 6-8 independent plants is given.

\section{Statistical analysis.}

Data significance was assessed using either Student's t test or one-way ANOVA and indicated by $* * P<0.05$ or $* * * P<0.01$ in the figure legends. 


\section{Results}

\section{BZR1 and PIFs have different role in PIF4 expression}

Both the G-box motif (CACGTG) and E-Box motif (CACATG ) are highly enriched in PIF4 target promoters (Oh et al., 2012; Zhang et al., 2013; Pfeiffer et al., 2014), suggesting that PIF4 binds to both to E-Box and G-Box. The promoter of PIF4 itself also contains a G-box and E-box (Ibañez et al., 2018) and it has been shown that tagged-PIF4 is bound to its own promoter in Chip experiments on DNA isolated from two week old plants (Oh et al., 2012) but not on DNA isolated from two day old seedlings (Oh et al., 2012; Pfeiffer et al., 2014). Binding of the BZR1 protein to the PIF4 promoter was confirmed by CHIP assay experiments with tagged BZR1 (Ibañez et al., 2018). Transcript comparison between the double mutant pifq; bzr1-1D and the bzr1-1D single mutant identified PIF regulated genes (Oh et al., 2012). Among these PIF regulated genes we identified PIF4, which shows a 3.5 fold upregulation in pifq. This suggests that PIFs are repressors of PIF4 expression. In contrast, for BZR1 it was shown that the interaction of BZR1 with the PIF4 promoter can stimulate transcription in an expression assay in protoplast from A.thaliana leaves (Ibañez et al., 2018). Combined, these results indicate that BZR1 and PIFs may have opposite roles in the regulation of PIF4 gene expression and that PIF4 gene activity may depend on the relative level of activated BZR1 versus PIF protein.

\section{PIF4 represses the positive action of BZR1 on pPIF4:LUC in transient assays}

To determine the effect of PIF4 on PIF4 promoter activity we used the transient expression system in N. benthamiana leaves. For this a pPIF4:LUC reporter construct was made using a 2487 bp promoter fragment of PIF4. This reporter was co-expressed with either a BZR1 effector construct (BZR1OE) or a PIF4 effector construct (PIF4OE). Results show that in the presence of a BZR1 overexpression construct, the pPIF4:LUC reporter activity is not significantly increased (Figure 1A). In contrast, when pPIF4:LUC is co-expressed with the PIF4 effector construct, this resulted in a significant down regulation of pPIF4:LUC activity (Figure 1B). The opposite action of BZR1 and PIF4 on PIF4 gene transcription, raises the question what happens to pPIF4:LUC activity when BZR1 and PIF4 are co-expressed with pPIF4:LUC? When the pPIF4:LUC reporter is co-expressed with both the BZR1 and PIF4 effector construct (both 
under control of the same 35S promoter), the expression of pPIF4:LUC is down regulated (Figure 1C). This indicates that the repressor activity of PIF4 on its own promoter is dominant over the activator activity of BZR1 on the PIF4 promoter. Subsequently we tested whether these interactions are also valid in stable transformed plants with ectopic expression of PIF4.

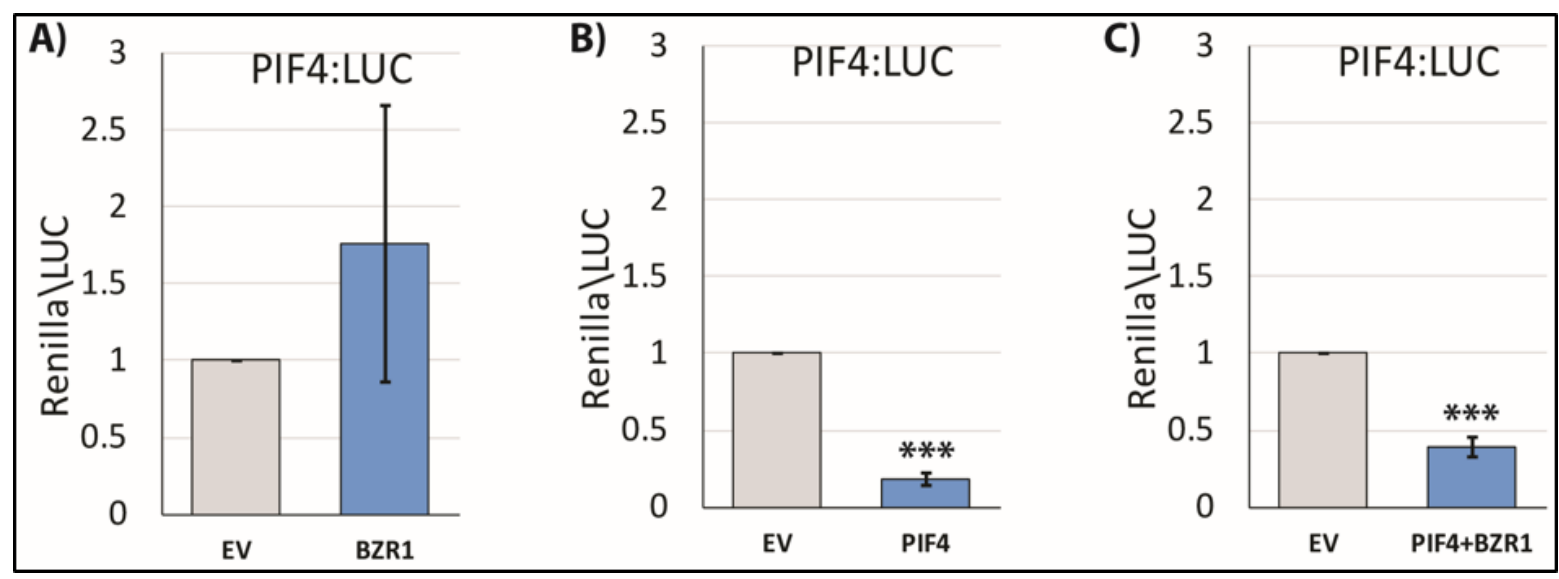

Fig.1. PIF4 suppresses PIF4 transcription in transient expression assays. pPIF4:LUC activity when coexpressed with empty vector effector construct (EV) or with the BZR1 effector construct (A), when co-expressed with empty vector effector construct (EV) or with the PIF4 effector construct (B) and pPIF4:LUC activity when co-expressed with 2x empty vector effector construct (EV) or with the BZR1 and PIF4 effector constructs (C). Each quantification is based on minimum of 5 agro-infiltrated leaves. Significant differences are indicated by $* * *(P=0.05)$.

\section{PIF4OE in Arabidopsis represses endogenous PIF4 expression.}

To determine the effect of ectopic expression of PIF4 in stable transformed plants we used the 35S:PIF4 effector construct for transformation of Arabidopsis Col-0 plants. Although most primary transformants with PIF4 overexpression (PIF4OE) did not set seeds we obtained three lines with PIF4OE. From these a homozygous line with PIF4OE was developed. However, we note that surviving progeny may not be representative of general PIF4OE. The expression level of the endogenous PIF4 gene was quantified by qPCR using primers specific for the 3'UTR of the endogenous PIF4 gene. Results show that in the homozygous PIF4OE plant the expression from the endogenous PIF4 gene is suppressed compared to that in control WT plants (Figure 2 ), indicating that also in stable transformed plants PIF4 is a negative regulator of its own expression. The transcription factor BZR1 is a major positive regulator of PIF4 expression (Ibañez et al., 2018) and in the mutant bzr1-1D, the BZR1 protein activity has been uncoupled 
from BR signaling (Ibañez et al., 2018). The PIF4OE construct was also transformed into the bzr1-1D mutant using the floral dip method and expression of endogenous PIF4 in T1 progeny plants was quantified. Results show that also in the bzr1-1D mutant background, the expression of endogenous PIF4 is suppressed by ectopic PIF4OE(Fig. 2).

\section{Endogenous PIF4}

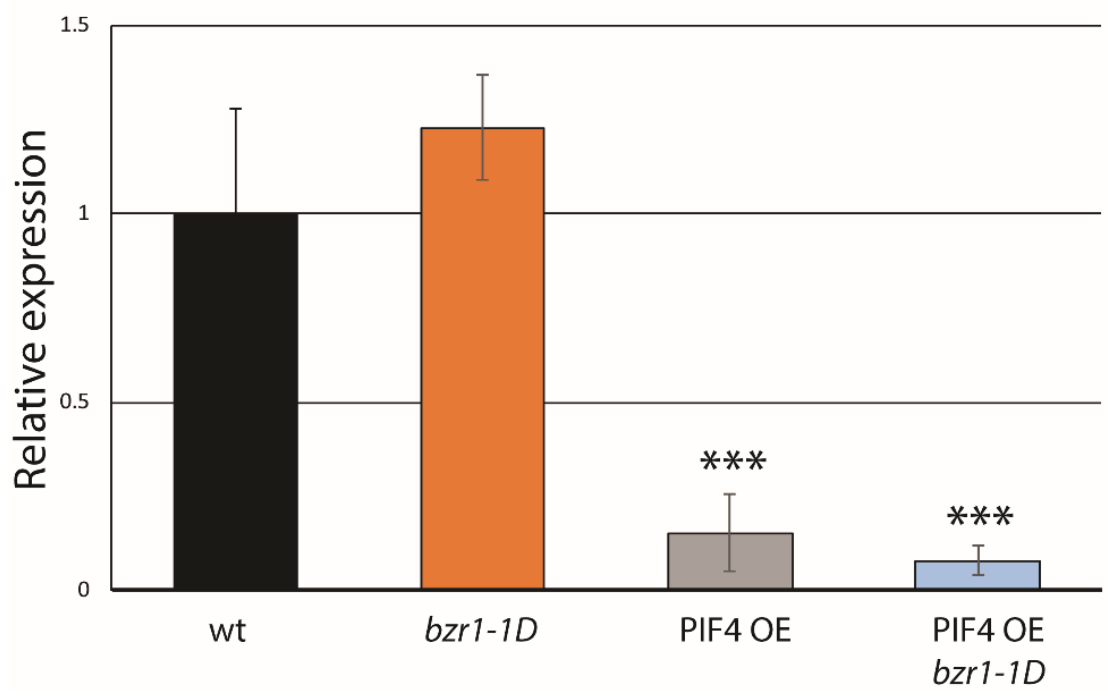

Figure 2. PIF4 OE in Arabidopsis suppresses endogenous PIF4 expression. The PIF4OE construct was introduced into WT and bzr1-1D by floral dipping and endogenous PIF4 mRNA levels were quantified in RNA isolated from leaves of 25-day old plants. Specific reverse primer were designed to 3UTR region of PIF4 transcript and used for the endogenous PIF4 gene expression. Significant differences are indicated by *** $(\mathrm{P}=0.05)$.

\section{pPIF4:LUC reporter activity is suppressed in PIF4OE plants}

To monitor PIF4 promoter activity in different backgrounds and at different temperatures we developed a pPIF4:LUC reporter line in A.thaliana (Col-0). Primary transformants were selected based on LUC activity in seedlings and in total 10 independent transformants were obtained. From these one homozygous line with representative pPIF4:LUC activity was developed and used for further study. Meantime, also a 35S:PIF4 OE transgenic line was generated in Arabidopsis (Col-0) by floral dipping. Approximately, 16 T0 seeds were selected based on the red seed coat marker present in the 35S:PIF4 effector construct. From the 16 T1 plants only three plants were able to produce seed, indicating that PIF4 overexpression has severe effects on seed set. From the three T2 plants a homozygous PIF4-OE line was developed 
and one of them crossed with pPIF4:LUC transgenic plant. Analysis of the pPIF4:LUC activity in the original WT pPIF4:LUC reporter plant and pPIF4:LUC/PIF4-OE plant shows that ectopic expression of PIF4 suppresses pPIF4:LUC activity (Figure 3), confirming that PIF4 is a negative regulator of its own promoter activity.

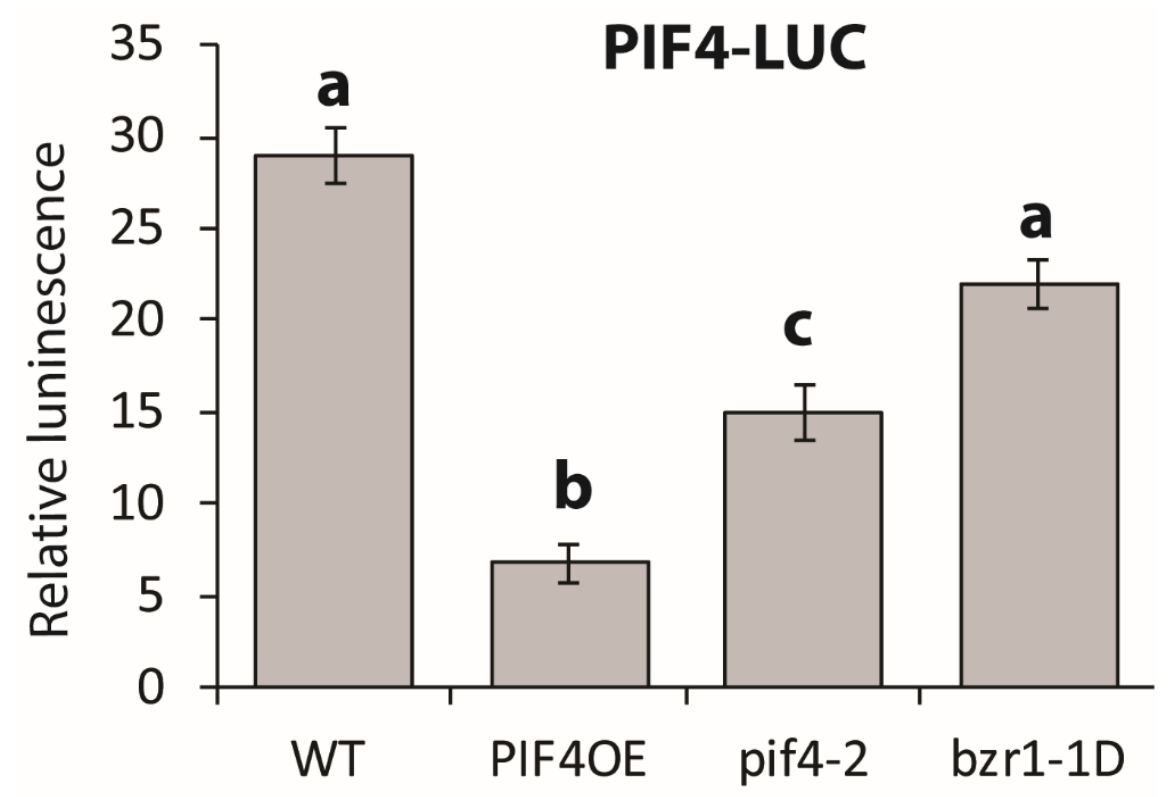

Figure 3. pPIF4:LUC activity in WT, PIF4OE, pif4-2 and bzr1-1D. Relative pPIF4:LUC activity quantified in 7 day old seedlings at $\mathrm{ZT}=9 \mathrm{hr}$. St error bars are shown, $\mathrm{N}=11$. Letters indicate statistically significant differences $(P=0.05)$ between WT and genotypes.

\section{pPIF4:LUC activity is reduced in the pif4-2 and bzr1-1D mutant background}

The pPIF4:LUC reporter was crossed into the pif4-2 mutant background to determine the effect of loss of PIF4 protein on PIF4 promoter activity. As results above identify PIF4 as a suppressor of its own promoter activity, the simple prediction was that pPIF4:LUC shows higher activity in a pif4-2 mutant background. However, results show that pPIF4:LUC activity is lower in pif4-2 (Figure 3). Similarly, the pPIF4:LUC reporter was crossed into the bzr1-1D mutant background (pPIF4:LUC brr1-1D), in which BZR1 activity is constitutively active due to a mutation in the BZR1 protein (Wang et al., 2002). However, the activity of pPIF4:LUC is not significantly higher in bzr1-1D compared to that in WT (Figure 3). This indicates that there are no simple predictions for PIF4 action on PIF4 promoter activity, most likely because PIF4 is affecting the different feedback interactions in different ways. 


\section{Transcriptional regulation of pPIF4:LUC as function of temperature}

To test the effect of warmth on PIF4 gene activity in WT and the different mutants, the different pPIF4:LUC reporter plants were grown under diurnal white light for 7 days. At day seven seedlings were placed in LUMINATOR for two days under mixed LED light at $22^{\circ} \mathrm{C}$. The LUC activity was measure every 30 minutes ( 7 min. exposure time) during the day and at night. The next day the temperature was raised from 22 to $27^{\circ} \mathrm{C}$ at $\mathrm{ZT}=0 \mathrm{hr}$. Figure 4 shows the pPIF4:LUC activity profile over a full day at 22 and full day at $27^{\circ} \mathrm{C}$. Results show that the switch to $27^{\circ} \mathrm{C}$ results in an immediate upregulation of pPIF4:LUC in all genetic backgrounds (WT, bzr1-1D, pif4-2 and in the PIF4OE line), however, the relative increase in pPIF4:LUC activity depends on the genetic background. Figure 4B shows the ratio between maximum pPIF4:LUC activity at $27^{\circ} \mathrm{C}$ and at $22^{\circ} \mathrm{C}$. Because the phase of pPIF4:LUC activity is slightly advanced in PIF4OE (Figure 4A), the peak activities at 22 and $27^{\circ} \mathrm{C}$ are compared at ZT= $9 \mathrm{hr}$ for WT, bzr11D and pif4-2 and at ZT=8 hr for PIF4OE. Results show that although PIF4 promoter activity is repressed by PIF4 overexpression, the relative response to warmth is the highest in the PIF4OE plant. Although the pPIF4:LUC activity is lower in pif4-2 and bzr1-1D, the relative response to warmth is about the same as in WT (Figure 4).
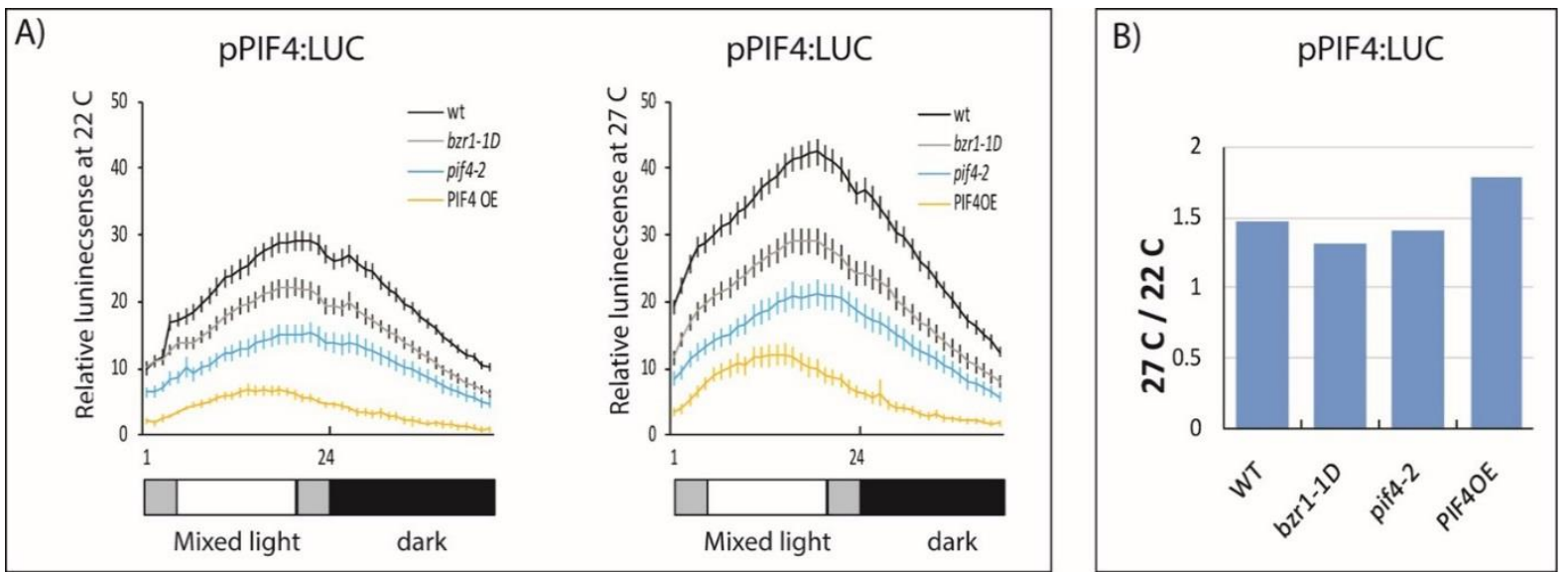

Figure 4. pPIF4:LUC activity in WT, bzr1-1D, pif4-2 and PIF4OE at $22^{\circ} \mathrm{C}$ and $27^{\circ} \mathrm{C}$. Seedlings were pregrown under diurnal white light for 7 days after which they were transferred to LUMINATOR for growth under mixed LED light for two days (results for second day shown). The third day the temperature was raised to $270 \mathrm{C}$ at $\mathrm{ZT}=0 \mathrm{hr}$. A). diurnal LUC profile under 22 and $27^{\circ} \mathrm{C}$. B: ratio of pPIF4:LUC at 27 and $22^{\circ} \mathrm{C}$. B). Ratio in average pPIF4:LUC activity at $27^{\circ} \mathrm{C}$ and $22^{\circ} \mathrm{C}$ for WT, pif4-2, bzr1-D and PIF4OE. Ratio's were calculated for peak time in expression ( $\mathrm{ZT}=8 \mathrm{hr}$ for PIF4OE, ZT=9 hr for others). Although PIF4OE represses the PIF4 promoter activity, the relative response to warmth is enhanced in PIF4OE. 


\section{Transcriptional regulation of pPIF4:LUC as function of light quality}

To test the role of PIF4 and BZR1 in pPIF4:LUC promoter activity as function of different light conditions the different pPIF4:LUC reporter plants were grown under diurnal white light for 7 days and at day seven seedlings were placed in LUMINATOR under mixed LED light at $22^{\circ} \mathrm{C}$ after adjusting to LUMINATOR for one day, the PPIF4:LUC activity was imaged every 30 min. for seedlings grown under 12 mixed/12D, followed by on day under $12 R / 12 D$, followed by one day under $12 \mathrm{FR} / 12 \mathrm{D}$ and finally under one day of 12B/12D. The full diurnal response under the different light conditions is shown in Figure S1 and the peak responses of pPIF4:LUC in WT, pif4-2, PIF4OE and in bzr1-1D under the different light colors are shown in Figure 5A. Results show that PIF4 gene expression is induced by R light, but remarkably, also by FR light. Figure $6 B$ shows the relative response to $R, F R$ and $B$ compared to the peak expression under mixed LED. These results indicate that the relative response to light quality is not much affected in the pif4-2 and bzr1-1D mutant. Remarkably, although expression of pPIF4 is lowest in PIF4OE plants, the relative response to FR is strongest in PIF4OE plants (Figure 5B). 

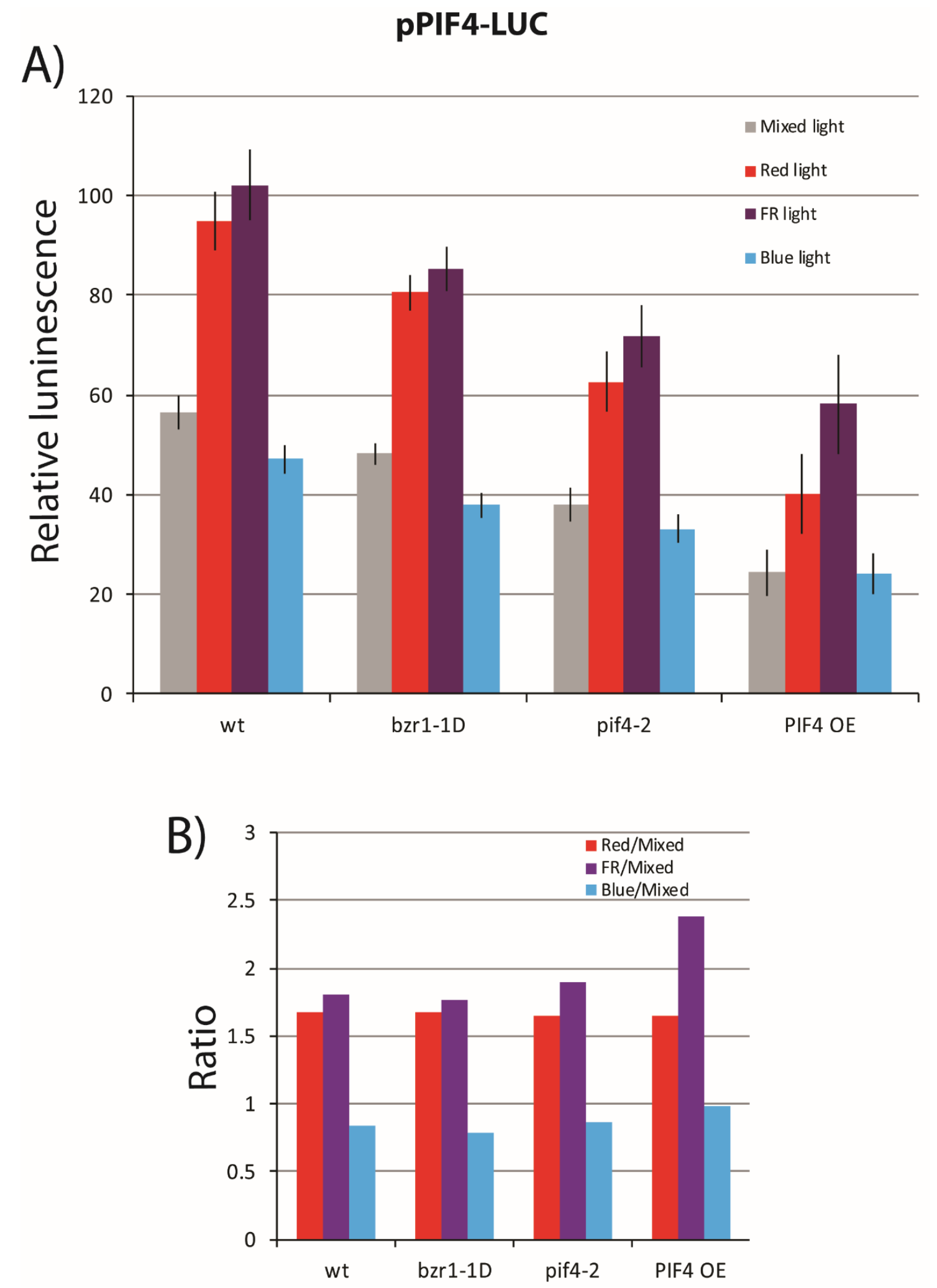

Figure 5. pPIF4:LUC under different light colors in WT, bzr1-1D, pif4-2 and PIF4OE. A) peak PIF4:LUC activity quantified under mixed $L E D$ at $Z T=9 \mathrm{hr}$, under $R$ at $Z T=12 \mathrm{hr}$, under $F R$ at $Z T=10 \mathrm{~h}$ ) and under $B$ at $Z T=12 \mathrm{hr}$. B) pPIF4:LUC response relative to mixed LED for plants under R (R:mixed), FR (FR:mixed) and B (B:mixed). 


\section{Discussion}

\section{PIF4 limits the feedforward regulation of PIF4 gene expression by BZR1}

During growth of plants internal hormonal signals and external environmental signals are integrated through the actions of the transcription factors PIF4 and BZR1 (Lucyshyn and Wigge, 2009; Choi and Oh, 2016). Understanding the regulation of PIF4 activity is therefore the basis for understanding how environmental signals may affect plant growth. While previous research has demonstrated the central role of BZR1 in transcriptional regulation of the PIF4 gene (Ibañez et al., 2018) the role of PIF4 itself in PIF4 gene expression has been largely ignored. BZR1 acts in an amplifying feed-forward loop on transcription of PIF4, in which the activity of PIF4 supposedly is kept under control by different post-transcriptional interactions with PIF4 protein. Here we show that the potential indirect feed-forward regulation of PIF4 gene transcription by BZR1 is actually also kept under control by the direct negative feedback of PIF4 on its own promoter activity. This is demonstrated by the suppression of endogenous PIF4 expression in plants by ectopic PIF4 overexpression, by the suppression of a pPIF4:LUC reporter activity in plants with ectopic PIF4 overexpression and by a transient expression assay in N.benthamiana leaves with the PPIF4:LUC reporter and PIF4 effector. Moreover, the transient assays also show that the PIF4 is still able to suppress pPIF4:LUC activity in the presence of BRZ1. The indirect positive feedback of BZR1 on PIF4 promoter activity involves several steps: PIF4 transcription, transcription of BR biosynthesis genes (Ibañez et al., 2018), BR biosynthesis, inhibition of BIN2 (He et al., 2002), dephosphorylation of BZR1 by PP2A (Tang et al., 2011), accumulation of the non-phosphorylated form of BZR1 in the nucleus and binding of BZR1 to the promoter of PIF4 and other target genes (He et al., 2005; Sun et al., 2010; Oh et al., 2012). In contrast the negative feedback of PIF4 on PIF4 promoter activity is more direct. Such positive and negative interactions on the same target may easily result in day-night cycles, as can be observed for PIF4 expression (Figure 4). Moreover, we provide evidence that PIF4 is part of the component that determines the phase of PIF4 expression as the day-night cycle in PIF4 expression show a late phase in the pif4-2 mutant and an early phase in the PIF4 overexpression line during the day, compared to the phase in WT (Figure 4). 


\section{PIF4 expression strongly induced under FR}

Our pPIF4:LUC reporter plants grown under either mixed LED, R, FR or B show that PIF4 expression is a function of light color. Most remarkably, PIF4 gene expression is strongly induced under R and FR light (Figure 5 and Figure S1). The strong induction under R may not be effective for PIF4 protein activity, as at the same time phytochromes are light activated and this may cause high turnover of PIF4 protein. However, the high expression of PIF4 under FR can result in high levels of PIF4 protein (Costa Galvao et al., 2018), because under FR phytochromes are not effectively activated. Therefore, either PIF4 is not a suppressor of its own gene activity under FR, or FR activates also some of the genes encoding components like CRY2 that have been shown to interfere with PIF4 activity or DNA binding (Wang et al., 2002; Box et al., 2015; Ma et al., 2016; Gangappa and Kumar, 2017). FR light induced PIF4 expression drops quickly in the dark and future experiments need to show what happens to PIF4 expression under continuous FR light.

\section{Lower expression of pPIF4:LUC in bzr1-1D explained?}

Even with the advanced insight that PIF4 is a suppressor of its own gene activity it is difficult to predict PIF4 gene activity in different mutant backgrounds. BZR1 is the positive factor for PIF4 transcription but BZR1 needs to be activated through BR signalling. Therefore, we expected that in the gain of function mutant bzr1-1D, with constitutively active BZR1, the PIF4 gene activity would be constitutively higher compared to that in WT. However, our results show that the pPIF4:LUC reporter activity in a bzr1-1D mutant background is not higher but slightly lower compared to that in WT. It could be that the higher BZR1 activity in bzr1-1D is affecting expression of one of the multiple components that lead to destabilisation or sequestering of PIF4 protein, such as PHYs, CRYs, DELLAs HFR1, HY5 or COP1. Indeed there is evidence that BR signalling affects phytochrome PHYB level, as the BR signalling mutant bri1116 accumulates higher level of PHYB protein than wild type (Sun et al., 2010). Moreover, BR signalling negatively regulates the transcription of several key components of the light response pathways, including photoreceptors phytochrome $B$ (PHYB), phototropin1, and the phytochrome-interacting proteins PIF3 and FHL and it is assumed that this is through 
BZR1/BES1 acting as suppressor of these genes (Sun et al., 2010). This could imply that an enhanced activity of BZR1 in bzr1-1D, may result in lower PHYB gene transcription and thus in lower PHYB protein levels. Because interaction of PHYB with PIF4 prevents binding of PIF4 to target promoters (Park et al., 2012) and PHYB suppresses PIF4 protein stability (Huq and Quail, 2002), lower PHYB protein levels in bzr1-1D could lead to higher PIF4 protein levels in bzr11D. The increased binding of PIF4 to its own promoter would then explain the modest activity of pPIF4:LUC in a bzr1-1D mutant background.

However, at the same time, enhanced BZR1 protein activity in bzr1-1D can result in a negative feedback on BR biosynthesis (Sun et al., 2010; Martínez et al., 2018). BR-signalling represses the kinase BIN2 activity on BZR1 and PIF4. The phosphorylation of both BES1/BZR1 and PIF4/PIF5 by BIN2 marks these transcription factors for proteasome degradation (He et al., 2002; Bernardo-Garcia et al., 2014). Therefore, if higher BZR1 activity in bzr1-1D results in lower BR signalling, one could expect higher BIN2 kinase activity and reduced PIF4 protein stability in bzr1-1D compared to that in WT (Bernardo-Garcia et al., 2014). As PIF4 is a negative regulator of PIF4 gene expression, a putative reduced PIF4 activity in bzr1-1D would result in higher PIF4 transcription level. Thus, current insights into the regulation of PIF4 provide opposing predictions for the activity of PIF4 in bzr1-1D: higher nuclear PIF4 protein stability due to lower PHYB expression and lower nuclear PIF4 stability due to higher BIN2 activity. The question is which of these two effects is stronger? Results of the pPIF4:LUC reporter in bzr1$1 D$ suggest a higher PIF4 protein activity in bzr1-1D (stronger suppression of pPIF4:LUC activity).

\section{Lower expression of pPIF4:LUC in pif4-2 mutant explained?}

If PIF4 is a negative regulator of its own expression, the simple prediction is that PIF4 gene activity is higher in the pif4-2 mutant, while pPIF4:LUC shows lower activity in pif4-2 (Figure 3). However, this may be explained if in the pif4-2 mutant background also activity of the positive factor BZR1 is affected. Indeed, PIF4 is a positive regulator for BR biosynthesis and signalling (Martínez et al., 2018). In pif4-2 there may thus be lower BZR1 activation and thus lower activation of the pPIF4:LUC reporter. In addition, it could be that PIF4 is involved in the 
expression of one of the multiple components that lead to destabilisation of PIF4 protein or sequestering of PIF4 protein, such as PHYs, CRYs, DELLAs HFR1, HY5 or COP1. Indeed, in a pif42 mutant the PHYB expression is reduced (Chapter 2.Figure 8). The lower PHYB transcription levels in 7-day old seedlings of pif4-2 could lead to more stable PIF4 protein, which is consistent with the observed higher suppression of pPIF4:LUC activity in pif4-2.

\section{Limited induction of pPIF4:LUC by PIF4 in transient expression assays}

Our transient expression assay with pPIF4:LUC and effector BZR1 shows only limited induction of LUC activity. Although qualitatively this is similar to the induction of PIF4 promoter activity by BZR1 in a protoplast assays of Col-0 mesophyll cells (Ibañez et al., 2018), the induction in the N.benthamiana leaf agro-infiltration assay (Figure 1) is much lower than the induction of PIF4 by BZR1 in the Arabidopsis protoplast assay (Ibañez et al., 2018). This may be caused by lower BR biosynthesis in agro-infiltrated leaves. Indeed, transcriptomic analysis of mRNA isolated from non-infiltrated $N$.benthamiana leaves and mRNA isolated from agro-infiltrated leaves show that in response to agro-infiltration most of the gene activity encoding enzymes that provide the precursors of brassinolide biosynthesis are significantly downregulated by agro-infiltration (Ting et al., 2015) (Figure S2).

\section{In conclusion}

We have shown that PIF4 protein itself provides a direct negative feedback of PIF4 gene transcription, to limit the indirect feed-forward regulation of PIF4 gene transcription by BZR1. However, with the pleiotropic effects of PIF4 and BZR1 on components that affect PIF4 protein activity it remains difficult to predict the dynamics of PIF4 gene activity under different conditions and in mutant backgrounds. The role of PIF4 in expression of PIF4 under FR light will need further investigation. The Luc reporter system would provide an ideal method to get dynamic and quantitative data in several mutant backgrounds, which could be an excellent base for a mathematical model that could help explain the result of positive and negative feedback loops. 


\section{References}

Bernardo-Garcia S, de Lucas M, Martinez C, Espinosa-Ruiz A, Daviere JM, Prat S (2014) BRdependent phosphorylation modulates PIF4 transcriptional activity and shapes diurnal hypocotyl growth. Genes Dev 28: 1681-1694

Bours R, Kohlen W, Bouwmeester HJ, van der Krol A (2015) Thermoperiodic control of hypocotyl elongation depends on auxin-induced ethylene signaling that controls downstream PHYTOCHROME INTERACTING FACTOR3 activity. Plant Physiol 167: 517530

Bours R, Muthuraman M, Bouwmeester H, van der Krol A (2012) OSCILLATOR: A system for analysis of diurnal leaf growth using infrared photography combined with wavelet transformation. Plant Methods 8: 29

Bours R, van Zanten M, Pierik R, Bouwmeester H, van der Krol A (2013) Antiphase light and temperature cycles affect PHYTOCHROME B-controlled ethylene sensitivity and biosynthesis, limiting leaf movement and growth of Arabidopsis. Plant Physiol 163: 882-895

Box MS, Huang BE, Domijan M, Jaeger KE, Khattak AK, Yoo SJ, Sedivy EL, Jones DM, Hearn TJ, Webb AAR, Grant A, Locke JCW, Wigge PA (2015) ELF3 controls thermoresponsive growth in Arabidopsis. Curr Biol 25: 194-199

Cagnola JI, Ploschuk E, Benech-Arnold T, Finlayson SA, Casal JJ (2012) Stem transcriptome reveals mechanisms to reduce the energetic cost of shade-avoidance responses in tomato. Plant Physiol 160: 1110-1119

Choi H, Oh E (2016) PIF4 Integrates Multiple Environmental and Hormonal Signals for Plant Growth Regulation in Arabidopsis. Molecules and Cells 39: 587-593

Costa Galvao V, Fiorucci A-S, Trevisan M, Franco-Zorrilla JM, Goyal A, Schmid-Siegert E, Solano R, Fankhauser C (2018) PIF transcription factors link a neighbor threat cue to accelerated reproduction in Arabidopsis. bioRxiv: 377663

Delker C, van Zanten M, Quint M (2017) Thermosensing Enlightened. Trends in Plant Science 22: $185-187$

Gangappa SN, Kumar SV (2017) DET1 and HY5 Control PIF4-Mediated Thermosensory Elongation Growth through Distinct Mechanisms. Cell Rep 18: 344-351

He JX, Gendron JM, Sun Y, Gampala SS, Gendron N, Sun CQ, Wang ZY (2005) BZR1 is a transcriptional repressor with dual roles in brassinosteroid homeostasis and growth responses. Science 307: 1634-1638

He JX, Gendron JM, Yang Y, Li J, Wang ZY (2002) The GSK3-like kinase BIN2 phosphorylates and destabilizes BZR1, a positive regulator of the brassinosteroid signaling pathway in Arabidopsis. Proc Natl Acad Sci U S A 99: 10185-10190

Huq E, Quail PH (2002) PIF4, a phytochrome-interacting bHLH factor, functions as a negative regulator of phytochrome $B$ signaling in Arabidopsis. EMBO J 21: 2441-2450

Ibañez C, Delker C, Martinez C, Bürstenbinder K, Janitza P, Lippmann R, Ludwig W, Sun H, James GV, Klecker M, Grossjohann A, Schneeberger K, Prat S, Quint M (2018) Brassinosteroids Dominate Hormonal Regulation of Plant Thermomorphogenesis via BZR1. Current Biology 28: 303-310.e303

Jung JH, Domijan M, Klose C, Biswas S, Ezer D, Gao M, Khattak AK, Box MS, Charoensawan V, Cortijo S, Kumar M, Grant A, Locke JC, Schafer E, Jaeger KE, Wigge PA (2016) Phytochromes function as thermosensors in Arabidopsis. Science 354: 886-889 
Li K, Yu R, Fan LM, Wei N, Chen H, Deng XW (2016) DELLA-mediated PIF degradation contributes to coordination of light and gibberellin signalling in Arabidopsis. Nat Commun 7: 11868

Lucyshyn D, Wigge PA (2009) Plant development: PIF4 integrates diverse environmental signals. Curr Biol 19: R265-266

Ma D, Li X, Guo Y, Chu J, Fang S, Yan C, Noel JP, Liu H (2016) Cryptochrome 1 interacts with PIF4 to regulate high temperature-mediated hypocotyl elongation in response to blue light. Proc Natl Acad Sci U S A 113: 224-229

Martínez C, Espinosa-Ruíz A, de Lucas M, Bernardo-García S, Franco-Zorrilla JM, Prat S (2018) PIF4-induced BR synthesis is critical to diurnal and thermomorphogenic growth. The EMBO Journal

Oh E, Zhu JY, Wang ZY (2012) Interaction between BZR1 and PIF4 integrates brassinosteroid and environmental responses. Nat Cell Biol 14: 802-809

Park E, Park J, Kim J, Nagatani A, Lagarias JC, Choi G (2012) Phytochrome B inhibits binding of phytochrome-interacting factors to their target promoters. Plant J 72: 537-546

Pedmale UV, Huang SS, Zander M, Cole BJ, Hetzel J, Ljung K, Reis PA, Sridevi P, Nito K, Nery JR, Ecker JR, Chory J (2016) Cryptochromes Interact Directly with PIFs to Control Plant Growth in Limiting Blue Light. Cell 164: 233-245

Pfeiffer A, Shi H, Tepperman JM, Zhang Y, Quail PH (2014) Combinatorial complexity in a transcriptionally centered signaling hub in Arabidopsis. Mol Plant 7: 1598-1618

Pham VN, Kathare PK, Huq E (2017) Phytochromes and Phytochrome Interacting Factors. Plant Physiology

Quint M, Delker C, Franklin KA, Wigge PA, Halliday KJ, van Zanten M (2016) Molecular and genetic control of plant thermomorphogenesis. Nat Plants 2: 15190

Raschke A, Ibanez C, Ullrich KK, Anwer MU, Becker S, Glockner A, Trenner J, Denk K, Saal B, Sun X, Ni M, Davis SJ, Delker C, Quint M (2015) Natural variants of ELF3 affect thermomorphogenesis by transcriptionally modulating PIF4-dependent auxin response genes. BMC Plant Biol 15: 197

Sun J, Qi L, Li Y, Chu J, Li C (2012) PIF4-mediated activation of YUCCA8 expression integrates temperature into the auxin pathway in regulating arabidopsis hypocotyl growth. PLoS Genet 8: e1002594

Sun Y, Fan X-Y, Cao D-M, He K, Tang W, Zhu J-Y, He J-X, Bai M-Y, Zhu S, Oh E, Patil S, Kim TW, Ji H, Wong WH, Rhee SY, Wang Z-Y (2010) Integration of Brassinosteroid Signal Transduction with the Transcription Network for Plant Growth Regulation in Arabidopsis. Developmental cell 19: 765-777

Tang W, Yuan M, Wang R, Yang Y, Wang C, Oses-Prieto JA, Kim TW, Zhou HW, Deng Z, Gampala SS, Gendron JM, Jonassen EM, Lillo C, DeLong A, Burlingame AL, Sun Y, Wang ZY (2011) PP2A activates brassinosteroid-responsive gene expression and plant growth by dephosphorylating BZR1. Nat Cell Biol 13: 124-131

Thingnaes E, Torre S, Ernstsen A, Moe R (2003) Day and night temperature responses in Arabidopsis: effects on gibberellin and auxin content, cell size, morphology and flowering time. Ann Bot 92: 601-612

Ting HM, Delatte TL, Kolkman P, Misas-Villamil JC, van der Hoorn RA, Bouwmeester HJ, van der Krol AR (2015) SNARE-RNAi results in higher terpene emission from ectopically expressed caryophyllene synthase in Nicotiana benthamiana. Mol Plant 8: 454-466 
Toledo-Ortiz G, Johansson H, Lee KP, Bou-Torrent J, Stewart K, Steel G, RodriguezConcepcion M, Halliday KJ (2014) The HY5-PIF regulatory module coordinates light and temperature control of photosynthetic gene transcription. PLoS Genet 10: e1004416

Wang Z-Y, Nakano T, Gendron J, He J, Chen M, Vafeados D, Yang Y, Fujioka S, Yoshida S, Asami T, Chory J (2002) Nuclear-Localized BZR1 Mediates Brassinosteroid-Induced Growth and Feedback Suppression of Brassinosteroid Biosynthesis. Developmental Cell 2: 505-513

Xu X, Paik I, Zhu L, Huq E (2015) Illuminating Progress in Phytochrome-Mediated Light Signaling Pathways. Trends Plant Sci 20: 641-650

Zhang X, Henriques R, Lin S-S, Niu Q-W, Chua N-H (2006) Agrobacterium-mediated transformation of Arabidopsis thaliana using the floral dip method. 1: 641-646

Zhang Y, Mayba O, Pfeiffer A, Shi H, Tepperman JM, Speed TP, Quail PH (2013) A quartet of PIF bHLH factors provides a transcriptionally centered signaling hub that regulates seedling morphogenesis through differential expression-patterning of shared target genes in Arabidopsis. PLoS Genet 9: e1003244

Zhu J-Y, Oh E, Wang T, Wang Z-Y (2016) TOC1-PIF4 interaction mediates the circadian gating of thermoresponsive growth in Arabidopsis. Nature Communications 7: 13692 


\section{Supplemental files}

\begin{tabular}{|c|c|c|c|}
\hline Primers name & Sequence & Used for & Ref \\
\hline PIF4-F & agcaaatctagaatggaacaccaaggttggag & Overexpression & In this study \\
\hline PIF4-R & agcaaagcggccgcctagtggtccaaacgagaacc & Overexpression & In this study \\
\hline BZR1-F & agcaaatctagaatgacttcggatggagctacg & Overexpression & In this study \\
\hline BZR1-R & agcaaagcggccgctcaaccacgagccttccc & Overexpression & In this study \\
\hline qPIF4-F & actcagatgcagccgatgg & qPCR for endogenic PIF4 & In this study \\
\hline qPIF4-R & acgtaatgaagttgcacgttt & qPCR for endogenic PIF4 & In this study \\
\hline qBZR1-F & gggaatctatcgctaagcaat & $\begin{array}{l}\text { qPCR for BZR1 gene, } \\
\text { mutant screening }\end{array}$ & In this study \\
\hline qBZR1-R & tctcttggaaggcagcagta & $\begin{array}{l}\text { qPCR for BZR1 gene, } \\
\text { mutant screening }\end{array}$ & In this study \\
\hline qPHYB-F & cgttgggtgttgctcctagt & qPCR for PHYB & In this study \\
\hline qPHYB-R & gataccccgcatcgcctaaa & qPCR for PHYB & In this study \\
\hline AT1G13320-F & taacgtggccaaaatgatgc & housekeeping & (Ibañez et al., 2018) \\
\hline AT1G13320-R & gttctccacaaccgcttggt & housekeeping & (Ibañez et al., 2018) \\
\hline pif4-2-LB & acctcctcaagtcatggttaagcctaagcc & Mutant screening & In this study \\
\hline pif4-2-RB & tccaaacgagaaccgtcggt & Mutant screening & In this study \\
\hline SAIL-LB2 & tagcatctgaatttcataaccaatctcgatacac & T-DNA screening & In this study \\
\hline
\end{tabular}

Table S1. List of primers were used in this study. 
pPIF4:LUC

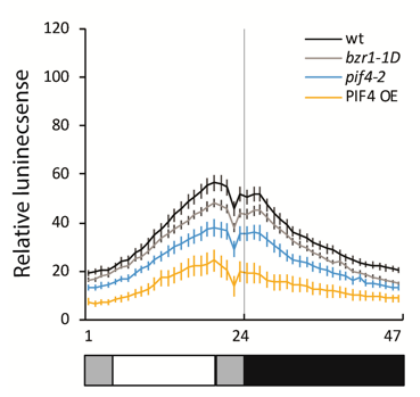

Mixed light

dark

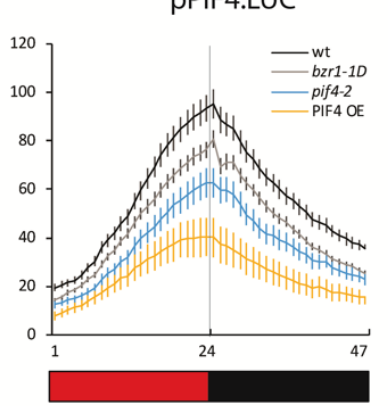

Red light

dark

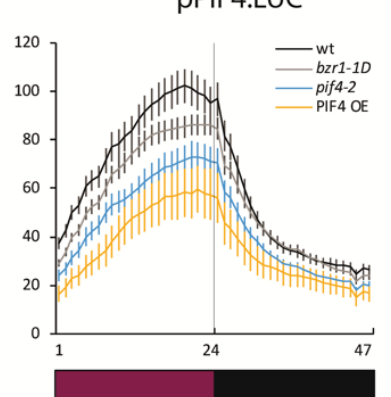

FR light
pPIF4:LUC

dark
pPIF4:LUC

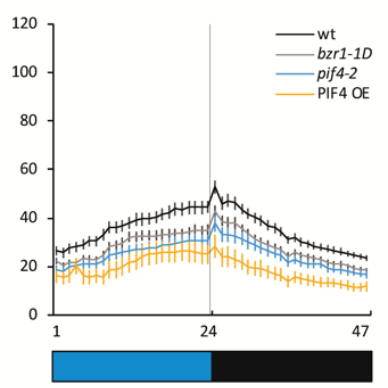

Blue light

Figure S1. A relative pPIF4:LUC activity in seedlings under different light conditions. pPIF4:LUC reporter

in WT, bzr1-1D, pif4-2 and PIF4 OE lines were stratified and germinated in growth cabinets under diurnal fluorescent WL (12L/12D). At 7 days after germination plants were sprayed with substrate luciferin $(1 \mathrm{mM})$ and one day later placed in LUMINATOR for adjustment under diurnal mixed R+B+FR for one day. Next three days, the light changed to pure R, FR and B light respectively. LUC activity was imaged every 30 min. minimum number of seedlings for each genotype: $n=18$ 


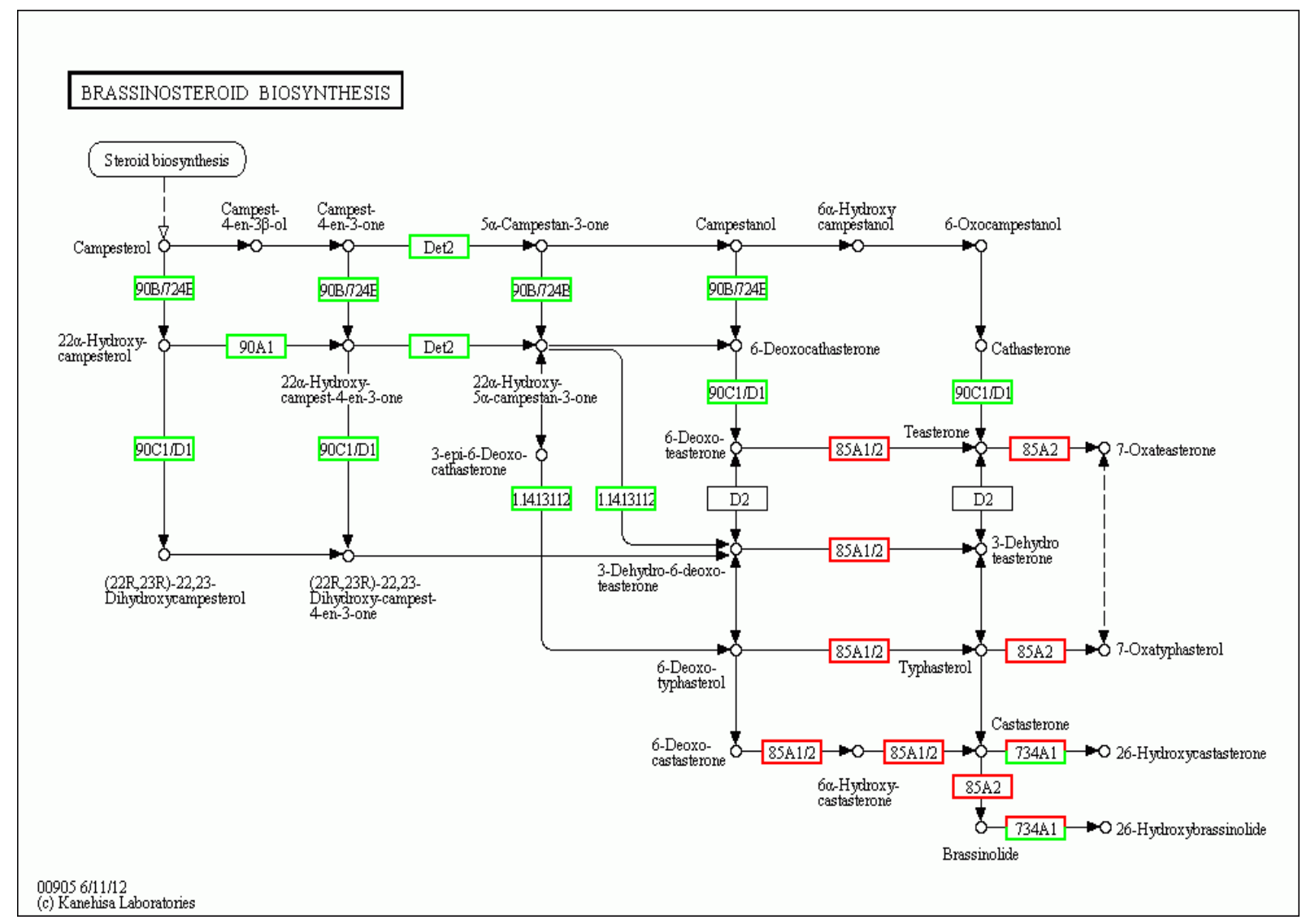

Figure S2. A reduced activity of genes involved in BR biosynthesis in agroinfiltrated leaves (Ting et al., 2015). Green: genes down regulated more than 2 -fold by agroinfiltration of vector expressing DsRED. Red: genes upregulated more than 2-fold by agroinfiltration with vector expressing DsRED. 



\section{CHAPTER-4}

A central role for MED25 in thermomorphogenesis

Umid Shapulatov

Martijn van Zanten

Mark van Hoogdalem

Mara Meisenburg

Alexander van der Hall

Iris Kappers

Alexander van der Krol

In preparation for publication 


\begin{abstract}
Thermo-morphogenesis is characterised by warmth-induction of auxin biosynthesis genes, including YUCCA8, resulting in strong hypocotyl elongation. The induction of YUCCA8 gene expression is mediated by transcription factors PIF4 and BZR1 and it has been shown that increased activity of PIF4 under warmth requires Histone Deacetylase 9 (HDA9). The Mediator complex functions as a bridge between transcription factors bound to specific promoter sequences and the basal transcription machinery containing RNA polymerase II. In plants the Mediator complex consists of 34 subunits of which the Mediator 25 (MED25) acts as hub for the transcriptional regulation of abiotic and biotic stress responses. A mutant of MED25 (pft12) shows reduced hypocotyl elongation and reduced expression of YUCCA8 under warmth, suggesting that MED25 affects PIF4 transcriptional activity at the YUCCA8 promoter. In a split luciferase assay we show that MED25 interacts with both PIF4, BZR1 and HDA9 in planta. Moreover, both PIF4 and HDA9 bind to the same polyQ domain of MED25. Genetic interaction studies indicate that pft1-2 and hda9-1 operate in the same pathway. In addition we demonstrate that MED25 enhances turnover of a HDA9-Luciferase fusion protein. Combined results uncover a central role for MED25 in thermo-morphogenesis induced elongation.
\end{abstract}

Key words: Mediator complex, thermomorphogenesis, elongation 


\section{Introduction}

Mediator of RNA polymerase II transcription (Mediator) is a conserved co-regulator of transcription conserved in yeast, metazoans and plants, consisting of a multi-subunit protein complex which comprises 25 subunits in budding yeast, 30 subunits in metazoans and 34 subunits in plants (Soutourina, 2018). The Mediator complex functions as a bridge between gene-specific regulatory proteins and the transcription initiation complex (TIC) containing RNA Pol II (Kidd et al., 2011; Samanta and Thakur, 2015). Of the different protein subunits forming the mediator complex, MED25/PFT1 is specific for metazoans and plants, but is absent in yeast and algae. Plant MED25 was initially identified as a gene affecting phytochrome signalling and flowering time and was therefore named PHYTOCHROME AND FLOWERING TIME1 (PFT1) (Cerdan and Chory, 2003). MED25/PFT1 interacts with a specific subset of transcription factors, as was determined by yeast two hybrid assays, by BiFC or by a split luciferase assay (see Table-S1). In plants, MED25 has been shown to convey transcriptional information related to methyl jasmonate (MeJA) signalling (Kidd et al., 2010; Cevik et al., 2012), flowering (Inigo et al., 2012), stress responses (Elfving et al., 2011) and floral organ size (Xu and Li, 2011). The MED25/PFT1 mutant (pft1-2) show reduced hypocotyl elongation under both red and far-red light (Kidd et al., 2009; Klose et al., 2012) and pft1-2 young rosette plants are smaller than WT plants (Cerdan and Chory, 2003). In contrast, the pft1-2 mutant has larger floral organ size, which was attributed to prolonged cell proliferation and elongation in petals of pft1-2 (Xu and $\mathrm{Li}, 2011)$. A role of MED25/PFT1 in hypocotyl elongation response as function of light intensity was confirmed by the dominant mutation PFT1 ${ }^{\text {eid3 }}$ (Klose et al., 2012). It was shown that PFT1 ${ }^{\text {eid3 }}$ enhances light sensitivity downstream of phytochrome A (phyA) and modulates phyB function, resulting in expression of light regulated genes in darkness (Klose et al., 2012). MED25/PFT1 is also required for the sugar-hypersensitive hypocotyl elongation phenotype of an UDP-arabinose synthesis mutant (hsr8-1) (Seguela-Arnaud et al., 2015).

Plant cell elongation responses are also strongly enhanced under high temperature (Gray et al., 1998; Quint et al., 2016). Auxin is an important hormone that mediates such thermo-morphogenesis responses (Gray et al., 1998; Delker et al., 2014; Bours et al., 2015; Ibañez et al., 2018) and it has been shown that MED25/PFT1 directly affects auxin signalling through interaction with Auxin Response Factors (ARFs) (Ito et al., 2016). Under low auxin 
levels, the activity of auxin response factors like ARF7 and ARF19 is blocked by the repressor Aux/IAA14 due to binding of another Mediator component MED13 (Ito et al., 2016). MED13 forms part of a CDK8 kinase domain of the Mediator complex. At low auxin, MED13 together with the co-repressor TOPLESS (TPL) inhibits the interaction between the ARF bound core Mediator complex and the transcription initiation complex (TIC) containing RNA Pol II. In high auxin, the Aux/IAA14 protein is targeted for degradation by the SCF ${ }^{\text {tir1 }}$ complex this results in the dissociation of TPL and MED13 from the complex. Subsequently, ARF7 and ARF19 interact with MED25 to recruit TIC containing RNA Pol II (Ito et al., 2016). The altered hypocotyl elongation responses of pft1-2 mutant could therefore be caused by the altered auxin signaling through ARFs in pif1-2. However, the MED25 protein interacts with transcription factors acting in auxin, jasmonic acid, ABA and ethylene hormone signalling pathways (Kazan, 2017). Therefore in the pft1-2 mutant, multiple hormone signalling pathways may be affected, all of which could influence hypocotyl elongation responses.

PIF4 and BZR1 are the central integrators in the transcriptional network regulating thermo-morphogenisis (Huq and Quail, 2002; Koini et al., 2009; Franklin et al., 2011; Kumar et al., 2012; Martínez et al., 2018). BZR1 was identified as a dominant regulator of PIF4 expression (Ibañez et al., 2018) and PIF4 mediates temperature-induced hypocotyl elongation by stimulating auxin biosynthesis via direct binding to the promoters of auxin biosynthesis genes, including YUCCA8 (Oh et al., 2012; Sun et al., 2012) and the auxin/indole-3-acetic acid (IAA) genes IAA19 and IAA29 (Sun et al., 2013). The PIF4 induced expression of auxin biosynthesis genes results in increased auxin levels and auxin-signaling, which mediates the cell elongation response (Bours et al., 2015; Ibañez et al., 2018). In addition, the interaction between PIF4 and BZR1 regulates BR biosynthesis (Martínez et al., 2018). Upon enhanced PIF4 activity through BZR1, BR synthesis is stimulated and the enhanced BR levels result in enhanced BR signalling, which in turn activates BZR1 in a feed forward loop (Ibañez et al., 2018). This positive feedback regulation of PIF4 transcription is supposedly kept under control by multiple factors acting on PIF4 protein stability (Zhang et al., 2017), on PIF4 DNA binding activity (Park et al., 2012) or by competition for PIF4 binding sites in the promoters of target genes of PIF4 (Nawkar et al., 2017). The activity of PIF4 is strongly regulated by phytochrome signalling because photo-activated phytochrome B (phyB) induces the degradation of PIF4 and PIF5 protein (Huq and Quail, 2002; Leivar et al., 2008). The pft1-2 mutant shows a reduced 
response under R and FR light, suggesting that MED25 may be involved in regulating PIF4 activity. However, PIF4 tested negative for interaction with MED25 in a yeast two hybrid assay (Ou et al., 2011).

Recently it was shown that thermo-morphogenesis requires histone deacetylation by Histone deacetylase 9 (HDA9) at the PIF4 target gene YUCCA8 (Tasset et al., 2018) (van der Woude et al., 2018 under review). Although histone deacetylation is typically associated with suppression of gene transcription, the activating role of HDA9 in PIF4 and downstream target gene activity under heat stress was linked to an effect on H2A.Z nucleosome dynamics in plants (van der Woude et al., 2018 under review). Indeed, heat stress has been shown to lead to the eviction of H2A.Z nucleosomes at thermo-responsive genes and this eviction improves chromatin accessibility for transcription factors and thus can lead to enhancement of gene expression (Kumar and Wigge, 2010; Cortijo et al., 2017). H2A.Z nucleosome dynamics may also be important for general response to external stimuli (Coleman-Derr and Zilberman, 2012; Sura et al., 2017). HDA9 is recruited to the promoter of PIF4 target genes by the POWERDRESS (PWR), which can bind to HDA9 (Tasset et al., 2018). In the model in which the eviction of H2A.Z at the YUCCA8 promoter is linked to the activity of HDA9 under warmth it is not clear how HDA9 is recruited to the YUCCA8 locus.

Here we investigated the role of MED25 in cell elongation responses under ambient temperature and warmth using hypocotyl elongation assays under ambient and high temperature conditions for WT, pft1-2 and MED25 overexpression plants. We demonstrate that MED25 can interact with both PIF4 and HDA9, but not with BZR1 in planta in a splitluciferase assay. Both PIF4 and HDA9 interact with the same C-terminal polyQ-domain of MED25, suggesting that the interaction with MED25 by PIF4 and HDA9 may be sequential. Moreover, we demonstrate that the interaction between MED25 and HDA9 results in destabilisation of HDA9. We speculate on a model in which MED25 recruits HDA9 and PWR at the YUCCA8 promoter, resulting in local histone deacetylation. Thus MED25 may be the missing factor that recruits HDA9 and PWR to targets of PIF4 under heat stress. The deacetylation activity of HDA9 on histones leads to eviction of H2A.Z, while the interaction between MED25 and HDA9 leads to HDA9 turnover. Subsequently this allows for binding of PIF4 to MED25, which then aids in the stimulation of gene expression under heat stress. 


\section{Materials and Methods}

\section{Plant material and growth conditions.}

Col-0 wild type genetic backgrounds were used for all experiments. Genotypes used in this paper are listed in Table 1 . The different T-DNA insertion mutants were obtained from the Nottingham Arabidopsis Stock Centre (www.arabidopsis.info) and homozygous mutant genotypes were confirmed by PCR using gene specific and T-DNA specific primers (Table S2). The double/triple and other hybrid lines were obtained by crossing in this study. Seeds were first incubated for $3-4$ days at $4^{\circ} \mathrm{C}$ in darkness. After cold treatment the water-imbibed seeds were sown onto rock wool. Arabidopsis plants were then grown in at $12 \mathrm{~L} 22^{\circ} \mathrm{C} / 12 \mathrm{D} 12^{\circ} \mathrm{C}$ on rock-wool on half strength Hoagland-nutrient solution.

\begin{tabular}{|c|c|c|}
\hline Genotypes & Description & Source \\
\hline pft1-2 & Endogenous MED25 KO & SALK_129555C \\
\hline 35S:HDA9-LUC ${ }^{W T}$ & 35S:HDA9-LUC reporter in Col-0 & Under review \\
\hline 35S:HDA9-LUC ${ }^{p f t 1-2}$ & 35S:HDA9-LUC wt crossed to pft1-2 mutant & this study \\
\hline 35S:HDA9-LUC & this study & this study \\
\hline
\end{tabular}

Table-1. Mutants and reporter lines used in this study.

Plasmid Constructs. The full length of PIF4, BZR1 and MED25 CDS was amplified from Col-0 cDNA library using a gene specific primer set including 5'overhang Xbal and 3'overhang Notl restriction sites (Table-S2). The expected PCR products were digested and ligated with T4 DNA ligase (\#M1801, Promega, Madison,USA) into pIV1A2.1 entry vector (www.impactvector.com) in the middle of CaMV35S promoter and RbcS1 terminator. For the gene promoter with LUC reporter constructs, the length of promoter sequence for YUCCA8 predicted according to reference (Sun et al., 2012) and the fragment amplified from gDNA by using 5'overhang Ascl and 3'overhang Xbal restriction site primers (Table-S2). The PCR products first digested with restriction enzymes and ligated into after removed CaMV35S promoter of pIV1A2.1 entry vector in front of LUC cDNA. The entry vectors were then cloned into binary vector named pKGW-Red Seed (www.gateway.psb.ugent.be/vector/) by LR reaction. Thereafter, pKGW-Red Seed/ expression and reporter vectors (Table-2) were transformed into A.tumefaciens AGL-0 stain which were used in transient expression assays in N.benthamiana or were used for 
Arabidopsis (Col-0) transformation using the floral dip method as described (Zhang et al., 2006). Transgenic TO seeds were identified by DsRed pigmentation of the seed coat or by LUC reporter activity. Seeds were harvested from T1 plants and homozygous plants were selected based on Mendelian- genetic segregation obtained the DsRed florescence at seed stage. For each representative homozygous line was selected from at least 10 primary transformants for further experiments.

Table-2. Expression constructs used in transient expression assays.

\begin{tabular}{|c|c|c|}
\hline Plasmid name & marker/ori/selection & Source \\
\hline 35S:cLuc & $\mathrm{Kan}^{\mathrm{R}} / \mathrm{pCAMBIA}$ & (Chen et al., 2008) \\
\hline 35S:nLuc & $K a n^{R} / p C A M B I A$ & (Chen et al., 2008) \\
\hline 35S:MED25-nLuc & $K a n^{R} / p C A M B I A$ & this study \\
\hline 35S:MED25 ${ }^{\mathrm{vWF}-\mathrm{A}}$-nLuc & $K a n^{R} / p C A M B I A$ & this study \\
\hline 35S:MED25 ${ }^{\mathrm{MD}}$-nLuc & $K a n^{R} / p C A M B I A$ & this study \\
\hline 35S:MED25 ${ }^{A C I D}$-nLuc & $K a n^{R} / p C A M B I A$ & this study \\
\hline 35S:MED25 ${ }^{\mathrm{GD}}$-nLuc & $K a n^{R} / p C A M B I A$ & this study \\
\hline 35S:cLuc-PIF4 & $K a n^{R} / p C A M B I A$ & this study \\
\hline 35S:PIF4-nLuc & $\mathrm{Kan}^{\mathrm{R}} / \mathrm{pCAMBIA}$ & this study \\
\hline 35S:cLuc-HDA9 & $K a n^{R} / p C A M B I A$ & this study \\
\hline
\end{tabular}

\section{Gene Expression Analysis by Quantitative Real-Time RT-PCR (qPCR).}

Total RNA were isolated from selected lines using homogenised young leaf tissues with InviTrap ${ }^{\circledR}$ Spin Plant RNA Kit and treated with Ambion ${ }^{\circledR}$ TURBO DNA-free Kit according to the manufacturer's instructions. cDNA synthesis was performed using the iScrip II mix reagent that included 10mM oligo (dT) primer according to the manufacturer's instruction (Bio-Rad, CA,USA). qPCR was carried out using iQ SYBR Green Super mix(Bio-Rad, CA,USA) on the CFX Connect Real Time System machine (Bio-Rad, CA, USA). The IPP2 or Actin genes were used as a reference gene for normalization of relative expression levels. Gene expression level is calculated from the average level detected in three biological replicate samples.

\section{Imaging and quantification of in planta Luciferase activity in Arabidopsis}


For the imaging of LUC-reporter activity in stable transformed Arabidopsis plants, the plants were pre-sprayed with $1 \mathrm{mM}$ D-luciferin (Duchefa Biochemie, Haarlem, NL) 24 hour prior to imaging to inactivate accumulated luciferase protein. Spraying with D-luciferin was repeated one hour before imaging. Imaging was with LUMINATOR consisting of an air-cooled $\left(-80^{\circ} \mathrm{C}\right)$ CCD Pixis 1024B camera system (Princeton Instruments, Massachusetts, USA) equipped with a 35mm, 1:1.4 Nikkon SLR camera lens (Nikon, Tokyo, Japan) fitted with a DT Green filter ring (Image Optics Components Ltd, Orsay, France) to block chlorophyll fluorescence. Exposure time for the LUC activity measurements is as indicated. In the diurnal LUC activity experiment, a spraying of D-luciferin was repeated once a day. For each reporter line the average LUC activity is given from at least 8 individual plants. Relative luminescence from LUC activity was quantified in Image J (Bethesda, Maryland, USA), using background subtraction.

\section{Transient expression assays using agro-infiltration in N.benthamiana leaves.}

To test the YUCCA8 transcription activity in N.benthamiana, the leaves were agro-infiltrated with the pYUCCA8:LUC-reporter and effector constructs 35S:PIF4, with or without the 35S:MED25 expression construct. Relative gene dosage of the different expression constructs was kept an equal by complementing the agro-infiltration with an agrobacterium containing an empty vector (EV) construct when necessary. Agro-infiltration also included a P19 expression construct to suppress gene silencing (Saxena et al., 2011) and Renilla luciferase construct as the control reporter. At least six leaves were infiltrated with per construct combination. After four days, the co-infiltrated leaves were harvested for further analysis. From each leaf, three leaf disks $(1 \mathrm{~cm}$ size) were taken for technical replicates. These leaf disk in $2 \mathrm{ml}$ tube is frozen with liquid nitrogen and subsequently grounded using metal bead in the shaking machine. $200 \mathrm{~mL}$ of passive lysis buffer was added to each sample, vortexed and frozen in liquid nitrogen. After 10-15 minutes on ice, samples were vortexed and spinned down on $14800 \mathrm{rpm}$ for 3 minutes. The supernatant was pipetted on a 96-wells plate for measurement in the Glomax machine. The subsequent measuring was done according to the specifications of the kit. Through co-infiltration with a vector containing the Renilla gene, it was possible to quantify the amount of protein inside leaf material labelled with luciferase. By dividing the value of Renilla by the value of luciferase to obtain a normalised value for 
luciferase. As all samples had 3 technical replicates, the average was taken from these samples.

\section{Split luciferase assays in N.benthamiana leaves}

The spit luciferase assays demonstrating the interaction between candidate proteins were performed by transient expression in N.benthamiana. Both pDEST-cLuc and pDEST-nLuc vectors we used as a backbone to modification (Chen et al., 2008). The Gateway cassette was amplified from pDEST-15 and cloned into nLUC and cLUC conventional vectors in Plant Developmental Biology, WUR and provided us. Modified destination vector was also included stop codon. Therefore, without stop codon of the coding sequence of MED25 and PIF4 were amplified from cDNA library used the listed primer sets (Table S1). The amplified PCR products were first cloned into the $\mathrm{PCR}^{\mathrm{TM}} 8$ TOPO entry vector (Invitrogen, Carlsbad, USA) by TOPO reaction and generated pENTR-MED25, pENTR-PIF4 and pENTR-HDA9. The positive insert of pENTR-MED25 were transferred into the destination vector PDEST-NLuc to generate MED25nLUC. Other constructs PENTR-PIF4, and pENTR-HDA9 were transferred into pDEST-cLUC to form CLUC-PIF4, and CLUC-HDA9, respectively, through one way Gateway LR recombination reactions (Table-2). Addition, PIF4-nLUC construct was also created with same way above to examining PIF4 and HDA9 interaction. As a negative control, the 35S:cLuc (pCAMBIA-cLuc) and 35S:nLuc (pCAMBIA-nLuc) expression constructs were used as described by Chen(Chen et al., 2008). Expression constructs were transformed to Agrobacterium tumefaciens (AGL-0) and grown at $27^{\circ} \mathrm{C}$ for $48 \mathrm{~h}$ and regrown $24 \mathrm{~h}$ in LB medium containing $10 \mu \mathrm{g} \mathrm{ml}^{-1}$ Rifampicin and $50 \mu \mathrm{g} \mathrm{ml}{ }^{-1}$ kanamycin. Agrobacterium tumefaciens cells were re-suspended in agro-infiltration buffer including $10 \mathrm{mM}$ MES (2-morpholino ethanesulfonic acid, Duchefa Biochemie, Haarlem, NL), $10 \mathrm{mM} \mathrm{MgCl}$, and $100 \mathrm{mM}$ acetosyringone (4'-hydroxy-3;,5;dimethoxyacetophenone, Sigma Aldrich, US) and incubated at room temperature for $3 \mathrm{~h}$ with low level shaking. For the assay an equal volume of two Agrobacterium stains (OD600=0.3) were co-infiltrated to 5 weeks old N.benthamiana leaves. At least 6 N.benthamiana leaves were used to each combination experiment. After $72 \mathrm{~h}$ post-agro infiltration the leaves were harvested. For the imaging of LUC activity the leaves were sprayed with $1 \mathrm{mM}$ D-luciferin at $24 \mathrm{hr}$ and $1 \mathrm{hr}$ before imaging. LUC activity was captured under LUMINATOR by seven minutes 
exposure. The relative LUC activity in the images was quantified by using Image J (Bethesda, Maryland, USA).

\section{Seedling hypocotyl elongation assays}

The seeds were surface sterilised using the gas-phase seed sterilisation protocol (Clough and Bent, 1998). After sowing the seeds on the $1 \% \mathrm{MS}$-agar plates, seeds were stratified at $4^{\circ} \mathrm{C}$ for 3 days in the dark. For germination, plates were transferred to growth chambers with specified light conditions at temperature of either $22^{\circ} \mathrm{C}$ or $27^{\circ} \mathrm{C}$. After one week, images were taken of the germinated seedlings and hypocotyl lengths were quantified from the images using Image J software.

\section{Statistical analysis.}

Comparison of means was analysed for statistical significance with a 2-sample t-test ( $P$ $<0.001)$.

\section{Results}

\section{MED25 affects hypocotyl elongation}

To investigate the role of MED25 in elongation responses in Arabidopsis, we measured hypocotyl elongation response of seedlings at ambient temperature $\left(22^{\circ} \mathrm{C}\right)$ and during warmth $\left(27^{\circ} \mathrm{C}\right)$ for WT and pft1-2. For this seeds were imbibed on MS-agar plates and stratified for 4 days in the dark at $4^{\circ} \mathrm{C}$ after which seeds were germinated under diurnal $12 \mathrm{WL} / 12 \mathrm{D}$ for 7 days at $22^{\circ} \mathrm{C}$ or $27^{\circ} \mathrm{C}$. Although pft1-2 hypocotyl elongation was not significantly different from WT at $22^{\circ} \mathrm{C}$, the pft1-2 mutant had a significantly shorter hypocotyl at $27^{\circ} \mathrm{C}$ (Figure 1), indicating an impaired thermo-morphogenesis response in pft1-2. These results identify a role for MED25 in warmth induced-hypocotyl elongation in Arabidopsis. The MED25 protein is known to interact with multiple bHLH transcription factors (Ou et al., 2011). Both BZR1 and PIF4 are a key transcription factors in thermo-morphogenesis and we therefore investigated whether the effect of MED25 on thermo-morphogenic response is caused by an effect of MED25 on PIF4 or BZR1 transcriptional activity. 


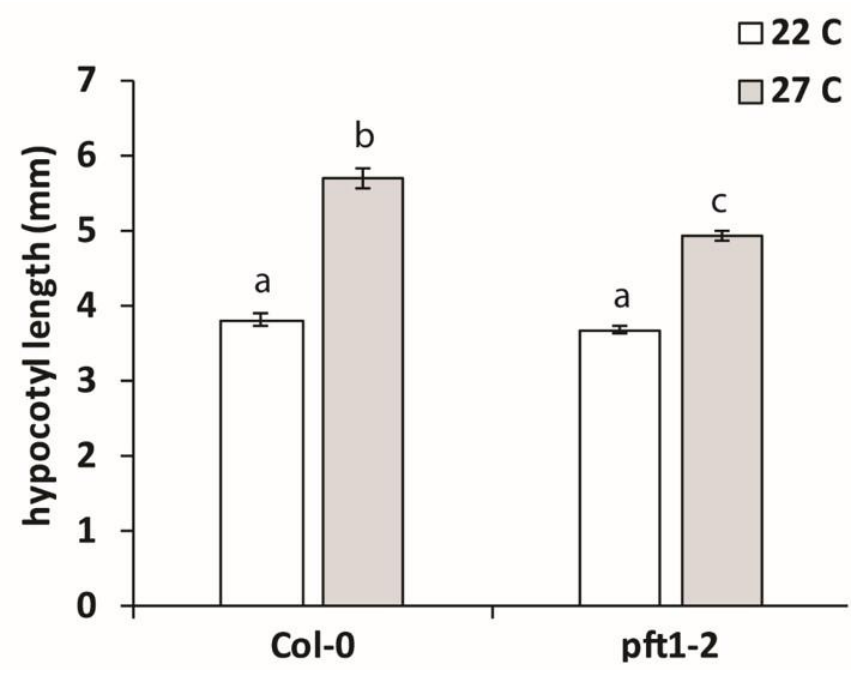

Figure 1. MED25 is required for full hypocotyl elongation at $27^{\circ} \mathrm{C}$. WT and pft1-2 plants were grown for 7 days on MS-agar plate at $22^{\circ} \mathrm{C}$ and $27^{\circ} \mathrm{C}$ in $12 \mathrm{~L} / 12 \mathrm{D}$ after which hypocotyl length was quantified. Data represents the mean $\pm S E$ ( $n=15$ seedlings). Different letters indicate significant differences $(P<0.05)$.

\section{Uncoupling of PIF4 expression with hypocotyl elongation in pft1-2 mutant.}

The transcription factor PIF4 acts upstream in the signal transduction pathway towards elongation. Since absence of MED25 reduces the warmth-induced elongation response, we tested whether MED25 influences the expression of PIF4 under warmth. For this purpose mRNA was isolated from WT (Col-0) and pft1-2 mutant plants grown for seven days at either $22^{\circ} \mathrm{C}$ or $27^{\circ} \mathrm{C}$. The sampling for RNA was at $6 \mathrm{PM}$ which is around the peak expression of PIF4 for plants grown under 12L/12D. Analysis of the PIF4 mRNA levels confirm the previously reported upregulation of PIF4 gene expression from $22^{\circ} \mathrm{C}$ to $27^{\circ} \mathrm{C}$ (Figure 2A). Surprisingly, PIF4 expression is upregulated at $22^{\circ} \mathrm{C}$ in pft1-2 compared to WT at $22^{\circ} \mathrm{C}$, even though hypocotyl length of pft1-2 is not significantly different from WT at $22^{\circ} \mathrm{C}$. Moreover, the expression of PIF4 was very strongly upregulated in pft1-2 under warmth, while the hypocotyl elongation of pft1-2 is smaller than in WT under warmth. Combined, the results indicate that MED25 affects PIF4 expression, but that the elevated expression level of PIF4 in pft1-2 is uncoupled from strong elongation responses. 

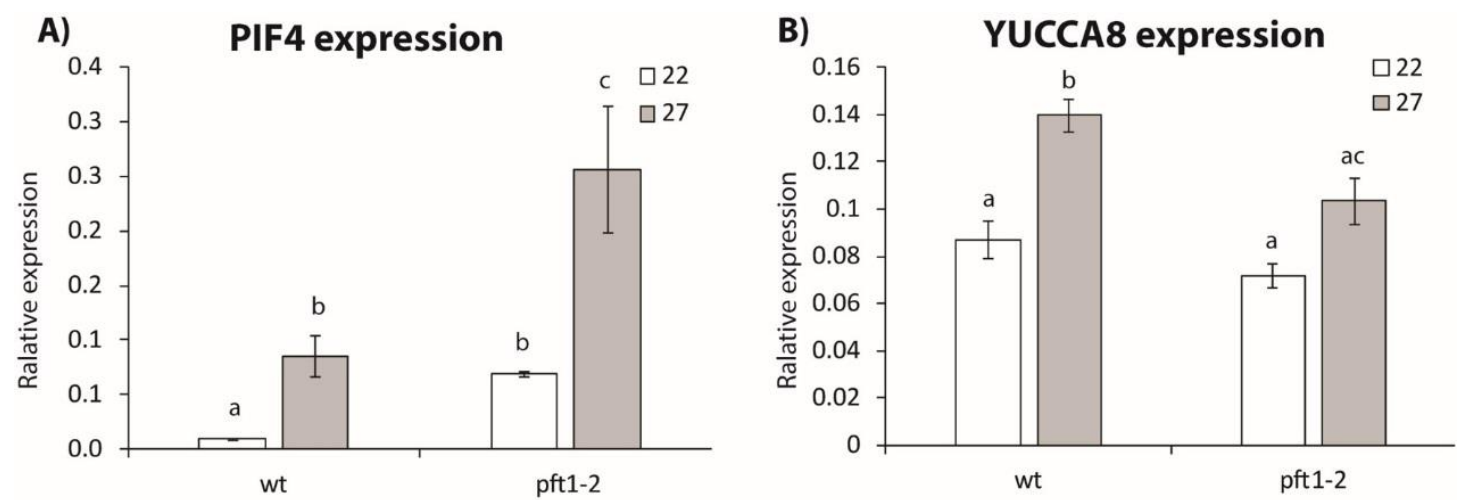

Figure 2. MED25 affects the expression of PIF4 (A) and YUCCA8 (B). Expression level of PIF4 and YUCCA8 was quantified by qPCR on mRNA isolated from WT and pft1-2 seedlings grown at $22^{\circ} \mathrm{C}$ or $27^{\circ} \mathrm{C}$.

In WT the hypocotyl elongation in response of warmth is the result of upregulation of PIF4 and BZR1 activity and subsequent upregulation of PIF4 target genes, such as the auxin biosynthesis gene YUCCA8 (Oh et al., 2012; Sun et al., 2012). Because the high expression of PIF4 in pft1-2 does not correlate with a strong elongation response we tested the expression of the PIF4 target gene YUCCA8 and of BZR1 to determine the cause of reduced hypocotyl elongation in pft1-2 under warmth. Surprisingly, results show that the expression of YUCCA8 is reduced in pft1-2 compared to that in $\mathrm{WT}$, both at $22^{\circ} \mathrm{C}$ and at $27^{\circ} \mathrm{C}$. The expression of BZR1 was not much affected in pft1-2, both at $22^{\circ} \mathrm{C}$ and $27^{\circ} \mathrm{C}$ (Figure 2B). The results indicate that PIF4 protein levels are uncoupled from the activity at the target gene expression YUCCA8 and suggests that MED25 is required for normal PIF4 protein activity. We therefore next tested whether PIF4 can interact with MED25.

\section{MED25 protein binding to PIF4 and BZR1 in in planta split luciferase assays}

In a previous screen using yeast two hybrid assays, PIF4 and BZR1 were not identified as targets for MED25 (Ou et al., 2011). However, it could be that either PIF4, BZR1 or the MED25 protein require plant specific modifications for an interaction between these transcription factors and MED25. Therefore the putative interaction between PIF4/BZR1 and MED25 was tested using an in planta split-luciferase binding assay (Chen et al., 2008). A split luciferase binding assay has been used before for MED25 to test the interaction between MED25 and TCP and COI1 (An et al., 2017; Liu et al., 2017). For this expression constructs were made encoding MED25 fused to the C-terminal end (CLUC) or N-terminal end (nLUC) of split Luciferase (MED25-CLUC and MED25-nLUC) and the expression constructs encoding PIF4-cLUC 
and BZR1-CLUC. The fusion proteins with split luciferase were expressed in N.benthamiana leaves by co-agro-infiltration of different combinations of the nLUC and CLUC constructs. Results show that the expression of the combination of MED25-nLUC and PIF4-cLUC in leaves resulted in a reconstitution of Luciferase activity, indicating an effective interaction between MED25 and PIF4 (Figure 3). Similarly, the expression of the combination of MED25-nLUC and BZR1-CLUC resulted in reconstituted luciferases activity, indicating that MED25 also can interact with BZR1 (Figure 3).

Having established that MED25 can bind to PIF4 and BZR1, we next tested to which domain of MED25 these two proteins bind. The MED25 protein contains multiple domains, each with specific functions (Figure 4C). The amino terminus MED25 has a conserved von Willebrand Factor Type A (vWF-A) domain. This domain mediates the interaction with the Mediator complex via binding the subunit MED16 (Yang et al., 2014). Transcription factors such as AP2/ERF, MYCs and suppressors such as JAZ proteins interact with the ACID (Activator Interacting Domain) domain of MED25 (see Table S1). At the C-terminus MED25 has a conserved glutamine rich (polyQ) tract named GD domain and it has been speculated that this domain is involved in transcriptional activation (Cerdan and Chory, 2003; Backstrom et al., 2007; Elfving et al., 2011). Moreover, the length of this polyQ rich region influences the effect of MED25 on flowering (Rival et al., 2014). The different domains of MED25 were cloned by adding an ATG start codon separately and fused to the nLUC sequence in expression constructs (vWF-A-nLUC; MD-nLUC; ACID-nLUC and GD-nLUC). These were tested in transient expression assays by co-agroinfiltration with either PIF4-CLUC or BZR1-CLUC. Results show that BZR1 cannot interact with the isolated domains of MED25 (Figure 3E), while PIF interacts with the GD domain of MED25 and weakly with the Acid domain (Figure 3D). This makes the interaction of PIF4 with MED25 different from other transcription factors that interact with MED25 (Figure S1). 
A)

\begin{tabular}{|c|c|}
\hline $\begin{array}{c}\text { nLUC } \\
+ \\
\text { LLUC }\end{array}$ & $\begin{array}{c}\text { MED25 -nLUC } \\
+ \\
\text { LLUC } \\
\text { MED25 -nLUC } \\
+ \\
\text { CLUC-PIF4 }\end{array}$ \\
\hline
\end{tabular}

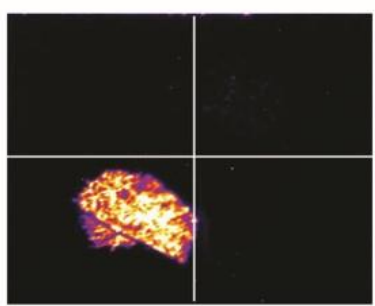

B)

\begin{tabular}{|c|c|}
\hline $\begin{array}{c}\text { nLUC } \\
+ \\
\text { CLUC }\end{array}$ & $\begin{array}{c}\text { MED25 -nLUC } \\
+ \\
\text { CLUC }\end{array}$ \\
\hline $\begin{array}{c}\text { MED25 -nLUC } \\
+ \\
\text { CLUC-BZR1 }\end{array}$ & nLUC \\
+ \\
CLUC-BZR1
\end{tabular}

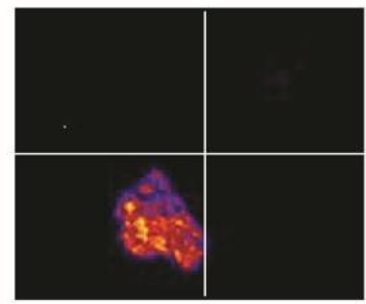

C)

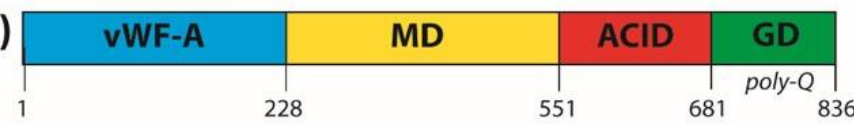

D)

\begin{tabular}{|c|c|}
\hline $\begin{array}{c}\text { MED } 25^{\text {vWF-A }}+\text {-nLUC } \\
+{ }_{\text {CLUC-PIF4 }}\end{array}$ & $\begin{array}{c}\text { MED25 } \\
+ \\
+ \\
\text { CLUC-PIF4 }\end{array}$ \\
\hline $\begin{array}{c}\mathrm{MED}_{25}{ }^{\mathrm{GD}} \text {-nLUC } \\
+ \\
\text { CLUC-PIF4 }\end{array}$ & $\begin{array}{c}\text { MED25 }{ }^{\text {ACID }} \text {-nLUC } \\
+ \\
\text { CLUC-PIF4 }\end{array}$ \\
\hline
\end{tabular}

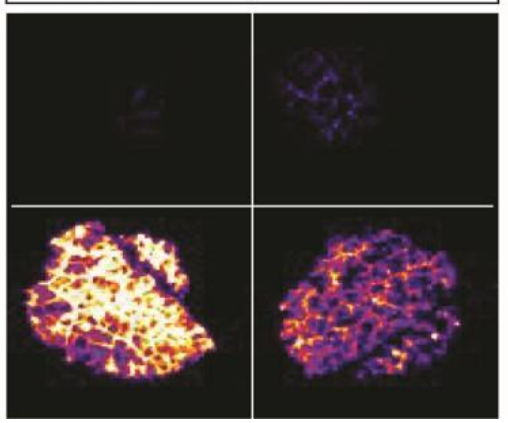

E)

\begin{tabular}{|c|c|}
\hline $\begin{array}{c}\text { MED } 25^{\text {vWF-A }} \text {-nLUC } \\
+ \\
\text { CLUC-BZR1 }\end{array}$ & $\begin{array}{c}\text { MED25 }{ }^{\text {MD }} \text {-nLUC } \\
+ \\
\text { CLUC-BZR1 }\end{array}$ \\
\hline $\begin{array}{c}\text { MED } 25^{\text {GD }} \\
+ \\
\text { CLULC-BZR1 }\end{array}$ & $\begin{array}{c}\text { MED } 25{ }^{\text {ACID }} \text {-nLUC } \\
+ \\
\text { CLUC-BZR1 }\end{array}$ \\
\hline
\end{tabular}

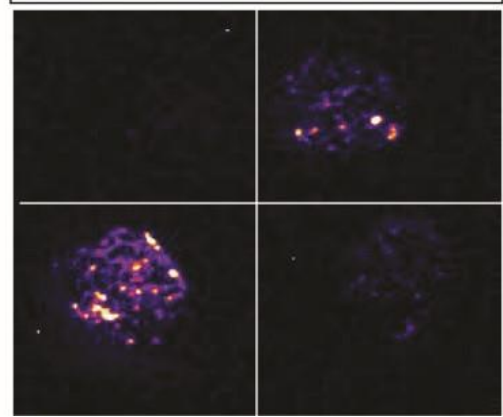

Figure 3. MED25 interacts with PIF4 and BZR1. (A) N.benthamiana leaves were infiltrated with different combinations of cLUC. nLUC, cLUC-PIF4 and MED25-nLUC expression constructs. Only the combination of cLUCPIF4+MED25-nLUC resulted in reconstitution of LUC activity. ). (B) N.benthamiana leaves were infiltrated with different combinations of cLUC. nLUC, cLUC-BZR1 and MED25-nLUC expression constructs. Only the combination of cLUC-BZR1+MED25-nLUC resulted in reconstitution of LUC activity. (C) the four sub domains of MED25 protein used in interaction studies. (D) N.benthamiana leaves were infiltrated with different combinations of MED25vWF-A-nLUC, MED25-MD-nLUC, MED25-GD-nLUC, MED25-ACID-nLUC and cLUC-PIF4. The combination of cLUCPIF4+MED25-GD-nLUC and cLUC-PIF4+ MED25-ACID-nLUC resulted in reconstitution of LUC activity. (E) N.benthamiana leaves were infiltrated with different combinations of MED25-vWF-A-nLUC, MED25-MD-nLUC, MED25-GD-nLUC, MED25-ACID-nLUC and cLUC-BZR1. None of the combination resulted in reconstitution of LUC activity. LUC activity images were taken three days post-agroinfiltration. 


\section{MED25 protein binds to HDA9 in in planta split luciferase assays}

Recently, a role for HDA9 in the heat induced hypocotyl elongation has been described, indicating that histone modifications are also part of the thermo-morphogenesis response (Tasset et al., 2018). It has been shown that HDA9 is recruited to the promoter of YUCCA8 and that de-acetylation by HDA9 at the YUCCA8 promoter is required for exchange of H2A.Z histones at the YUCCA8 promoter at high ambient temperature (van der Woude et al., 2018, under review). However, it is at present not clear how HDA9 is recruited to the YUCCA8 promoter. Here we tested whether MED25 can recruit HDA9 to the YUCCA8 promoter, based on an interaction between MED25 and HDA9. For this we used again the split luciferase assay in N. benthamiana. Expression constructs encoding PIF4-nLUC vs CLUC-HDA9, MED25-nLUC vs cLUC-HDA9 were made. Co-expression of MED25-nLUC + cLUC-HDA9 resulted in strong luminescence in N.benthamiana leaves, while cells co-expressing a combination of PIF4nLUC+CLUC-HDA9 fusion proteins did not result in reconstituted LUC activity (Figure 4A). This confirms that MED25 and HDA9 can interact in planta, but that HDA9 does not interact with PIF4. MED25 domain mapping with split luciferase assays revealed that the MED25-HDA9 interaction is with the GD and ACID domains of MED25 (Figure 4B). This is different from the interaction of MED25 with another histone modifying enzyme, HAC1, which binds to the combined MD+ACID domain of MED25 (An et al., 2017). However, the binding of HDA9 to GD and ACID domain is similar to the interaction of PIF4 with MED25 (Figure 3D). 


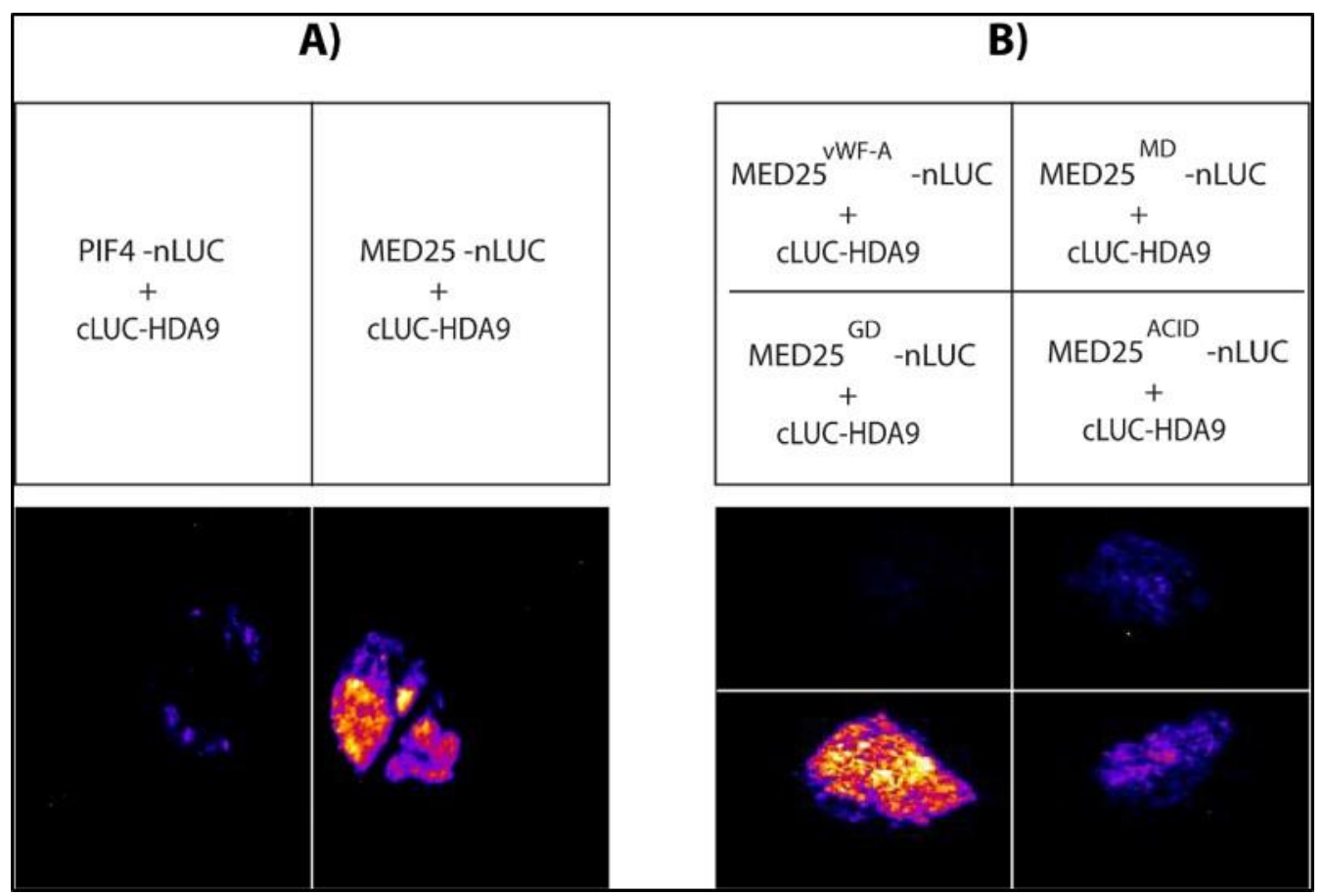

Figure 4. MED25 interacts with HDA9. (A) N.benthamiana leaves were infiltrated the combination PIF4-nLUC +cLUC-HDA9 or MED25-nLUC+ CLUC-HDA9. Only the combination of MED25-nLUC+ cLUC-HDA9 resulted in reconstitution of LUC activity. (B) N.benthamiana leaves were infiltrated with different combinations of MED25vWF-A-nLUC, MED25-MD-nLUC, MED25-GD-nLUC, MED25-ACID-nLUC and CLUC-HDA9. The combination of CLUC-HDA9 with MED25-GD-nLUC. MED25-ACID-nLUC and MED25-MD-nLUC resulted in reconstitution of LUC activity. LUC activity images were taken three days post-agroinfiltration.

\section{MED25 affects HDA9 protein stability}

It has been shown that the HDA9 protein is stabilised during heat stress (van der Woude, 2018). Since we have established that MED25 can bind to HDA9 we investigated whether this interaction affects the HDA9 protein stability. The HDA9 protein stability is measured using plants transformed to express an HDA9-LUC fusion protein (35S:HDA9-LUC) (van der Woude et al., 2018 under review). A representative homozygous WT reporter line expressing 35S:HDA9-LUC was crossed to pft1-2 and a line expressing a 35S:MED25 expression construct (MED25OE). From the F2 progeny plants homozygous for the 35S:HDA9-LUC construct and the pft1-2 mutation or homozygous for 35S:HDA9-LUC and MED25OE were selected for further investigation. The HDA9-LUC activity was measured in seven day old seedling in LUMINATOR under diurnal $12 \mathrm{~L} / 12 \mathrm{~S}$ mixed LED (R+FR+B) at $22^{\circ} \mathrm{C}$ (Figure 5). Images of LUC activity were 
captured every $30 \mathrm{~min}$. by a seven min. exposure. The relative LUC activity per seedling is quantified by image analysis using Image J. Results show that in the pft1-2 background the average activity of HDA9-LUC is higher than in WT, especially during the dark period. This suggests that MED25 contributes to a destabilisation of HDA9-LUC protein, especially in the dark. The destabilising effect of MED25 on HDA9-LUC is confirmed by the activity of HDA9LUC in the MED25OE background, which is barely above background, both during day and night (Figure 5A). Next, we determined the effect of MED25 on HDA9-LUC activity at $27^{\circ} \mathrm{C}$. The seven-day old reporter seedlings were grown at $22^{\circ} \mathrm{C}$ in LUMINATOR and after 2 hours at $22^{\circ} \mathrm{C}$ the temperature was raised to $27^{\circ} \mathrm{C}$. LUC activity was imaged every 30 minutes. Results show an immediate effect on LUC activity, indicating an immediate increase of HDA9 protein stability (Figure 5B). We note that the initial rate of increase in LUC activity is higher in the absence of MED25 (in pft1-2) than in WT. Curiously, the LUC activity increases near the end of the day and increases even further during the night to reach a maximum at three hours after onset darkness at $27^{\circ} \mathrm{C}$.

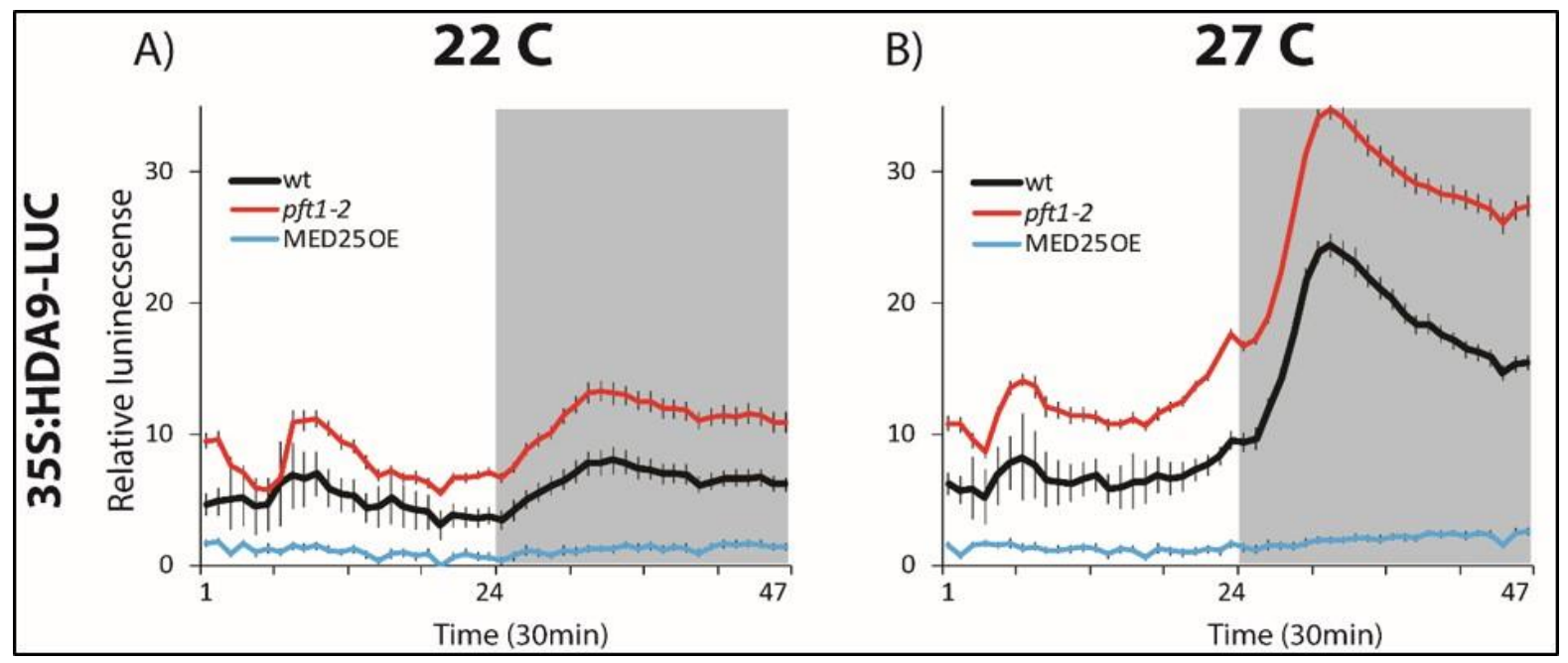

Figure 6. HDA9-LUC stability is affected by MED25. Six day old seedlings of Arabidopsis WT, pft1-2 or MED25OE plants expressing the same 35S:HDA9-LUC reporter were sprayed with substrate luciferin (1 mM) and placed in LUMINATOR under $12 \mathrm{~L} / 12 \mathrm{D}$ at $22^{\circ} \mathrm{C}$. LUC activity was quantified every $30 \mathrm{~min}$ (10 min image capturing). (A) result after one day adaptation at $22^{\circ} \mathrm{C}$. (B) temperature was raised from $22^{\circ} \mathrm{C}$ to $27^{\circ} \mathrm{C}$ at $\mathrm{ZT}=3 \mathrm{hr}$. At least 18 seedlings tested for each reporter lines. Error bars represent mean $\pm \mathrm{SE}$.

In contrast, the LUC activity is not increased at all at $27^{\circ} \mathrm{C}$ in the MED25OE background, indicating that the effect of MED25 is a dominant over the stabilising effect of heat on HDA9. Because MED25 negatively affects HDA9 protein stability we checked whether increased 
HDA9 stability coincides with a decrease in MED25 gene expression. For this we retrieved the diurnal expression patterns of HDA9 and MED25 as function of temperature and light from Diurnal database (Mockler et al., 2007). These data show that expression of MED25 is increasing up to 2-fold during high temperature (Figure S2A), while expression of MED25 slightly decreases in the light at high temperature (Figure S2B). Thus, the stabilisation of HDA9 at high temperature in WT cannot be explained by strong downregulation of MED25 at high temperature. Rather, the stabilisation of HDA9 at high temperature is despite upregulation of MED25 under high temperature. Combined these findings identify MED25 as a destabiliser for HDA9 protein.

\section{Discussion}

\section{MED25 has a central role in thermo-morphogenesis responses}

Here, we have uncovered a central role for MED25 in thermo-morphogenesis. This role of MED25 in thermo-morphogenesis is demonstrated by (1) a reduced hypocotyl elongation of the MED25 mutant pft1-2 at warmth (Figure 1), (2) the interaction of MED25 with the key transcription factors of the thermo-morphogenesis response, BZR1 and PIF4 (Figure 3). (3) the reduced activity of PIF4 at the PIF4-target YUCCA8 (Sun et al., 2012; Ibañez et al., 2018) under warmth in pft1-2 (Figure 2). (4) the regulation of YUCCA8 during warmth requires the activity of the histone deacetylase enzyme HDA9 (van der Woude et al., 2018 under review) and we demonstrate that MED25 can interact with HDA9 (Figure 4). (5) and the fact that MED25 is involved in stability of the HDA9 protein (Figure 5).

The central role of MED25 in the regulation of YUCCA8 is explained by the fact that MED25 potentially brings together three key players in the regulation of gene expression related to warmth induced elongation (e.g. YUCCA8): the transcription factors PIF4 and BZR1 and the histone deacetylase activity encoded by HDA9. The interaction of PIF4, BZR1 and HDA9 with the YUCCA8 promoter has been demonstrated by CHIP analysis (Franklin et al., 2011; Sun et al., 2012; Tasset et al., 2018), however, the YUCCA8 promoter was also identified as target in CHIP and transcriptome experiments with ARF6, PIF1, PIF3 and PIF5 (Oh et al., 
2014; Kim et al., 2016), suggesting that overall regulation of YUCCA8 may be far more complex. Future analysis using CHIP RNA seq with a tagged MED25 may confirm that MED25 can also be found at the YUCCA8 promoter.

\section{Relevance of MED25 interaction with PIF4 and BZR1 is gene dependent}

Our results show that MED25 can interact with BZR1, PIF4 (Figure 3) and HDA9 (Figure 4). In the context of regulation of YUCCA8 expression the interaction of MED25 with PIF4 and HDA9 seem to be relevant, as CHIP experiments have demonstrated that PIF4 and HDA9 are associated with the YUCCA8 locus (Tasset et al., 2018). However, CHIP experiments with tagged BZR1 have not identified YUCCA8 as target, so the interaction between MED25 and BZR1 may not be relevant for the regulation of YUCCA8. In contrast, the PIF3, PIF4 and PIF5 genes all were identified as target for BZR1 and PIF4 in CHIP experiments (Lee et al., 2007; Hornitschek et al., 2012; Oh et al., 2012; Oh et al., 2014; Pfeiffer et al., 2014), suggesting that the interaction of BZR1 with MED25 and the interaction of PIF4 with MED25 may be relevant for the expression of these PIF genes. Of the phytochrome genes only PHYB was identified as target for both BZR1 and PIF4 in CHIP experiments, while PHYD was only identified as target for PIF4 (Table 3).

\begin{tabular}{l|ccc} 
& $\begin{array}{c}\text { BZR1 (Oh et al., 2012) } \\
\text { (5 days) }\end{array}$ & $\begin{array}{r}\text { PIF4 (Pfeiffer et al., 2014) } \\
\text { (2 days) }\end{array}$ & $\begin{array}{c}\text { PIF4 (Oh et al., 2012) } \\
\text { (14 days) }\end{array}$ \\
\hline BZR1 & $\mathrm{x}$ & $\mathrm{x}$ & $\mathrm{x}$ \\
HY5 & & $\mathrm{x}$ & $\mathrm{x}$ \\
PIF1 & $\mathrm{x}$ & $\mathrm{x}$ \\
PIF3 & $\mathrm{x}$ & $\mathrm{x}$ \\
PIF4 & $\mathrm{x}$ & $\mathrm{x}$ \\
PIF5 & $\mathrm{x}$ & $\mathrm{x}$ \\
MED25 & $\mathrm{x}$ & $\mathrm{x}$ \\
YUCCA8 & & $\mathrm{x}$ & $\mathrm{x}$ \\
PHYA & $\mathrm{x}$ & & \\
PHYB & & & \\
PHYC & & & \\
PHYD & & & \\
PHYE & & &
\end{tabular}

Table 3. CHIP results with BZR1 and PIF4 for selected target genes. Data obtained from published CHIP experiment with BZR1 (Oh et al., 2012), with PIF4 (Oh et al., 2012; Pfeiffer et al., 2014). 


\section{MED25 interacts with Histone acetylase (HAC1) and Histone deacetylase (HDA9)}

In the context of Jasmonic Acid (JA) signalling it has been described that MED25 binds to both COI1 (the receptor for the active form of JA) and to the transcription factor MYC2. MED25 thus links the Mediator complex and COI1 to MYC2 target genes (An et al., 2017). Moreover, in the context of JA signaling, it has been shown that MED25 can bind to HISTONE ACETYLTRANSFERASE1 (HAC1) and that HAC1 interacts with the combined ACID+MD domain of MED25 (An et al., 2017), similar as was shown here for the interaction of PIF4 with MED25 (Figure 3). This interaction plays an important role in JA signaling by selective acetylation of histones at MYC2 target promoters allowing transcription of MYC2 target genes.

\section{Sequential binding of HDA9 and PIF4 at the YUCCA8 locus?}

We found that MED25 can interact with HDA9 through the GD and ACID domain of MED25 (Figure 4B). These are the same domains to which can bind PIF4 (Figure 3D) and raises the obvious question whether these proteins can bind simultaneously to MED25. This needs to be tested in the future in competition assays with PIF4 and HDA9 with MED25. However, the interaction between MED25 and HDA9 leads to destabilisation of HDA9. Therefore it is also possible that binding of HDA9 to MED25 is followed by binding of PIF4 to MED25, after degradation of HDA9.

\section{MED25 recruits different histone modifying activities}

The full Mediator complex is known to interact with different chromatin modifying protein complexes, such as SWI/SNF and histone deacetylases and acetylases (Sharma and Fondell, 2002; Malik and Roeder, 2010). The paralog of MED25 in human can interact with histone acetylases (Black et al., 2006). Moreover, in mammalian cells Mediator can counteract Polycomb dependent repression of gene activity though the MED25 subunit, which blocks the binding of Polycomb Repressor Complex2 (PRC2) to specific target genes (Englert et al., 2015). Assuming a conserved function between human MED25 and plant MED25, this suggests that MED25 in plants may also interact with chromatin modifying complexes. Indeed, in the 
context of JA signalling, MED25 has been shown to interact with COI1 and histone acetylase HAC1 (An et al., 2017). Interestingly, it also has been shown that COI1 interacts with the histone deacetylase HDA6 (Devoto et al., 2002), indicating that regulation of genes targeted by COI1 signaling may involve both histone acetylase and histone deacetylase activities, perhaps acting in a sequential way. Recently, it is revealed HDA9 and HDA6 genetically interacts to control of auxin signalling genes for elongation of silique valve cells (Yuan et al., 2018).

In the context of auxin signalling MED25 can recruit the CDK8 kinase module (CKM) to change the module composition of Mediator. CKM contains HEN3, the transcription corepressor LEUNIG, but also histone deacetylase HDA19 (Gonzalez et al., 2007). It was not tested whether MED25 interacts directly with HDA19 in this context.

In the context of the PIF4/BZR1 target gene YUCCA8 we now have shown that MED25 plays a role in the recruitment of HDA9. Previously it was shown that the activity HDA9 at the YUCCA8 locus is required for a normal gene induction under warmth (van der Woude et al., 2018 under review). However, this activity of HDA9 automatically implies that at some point also histone acetylase activities may be recruited to the YUCCA8 locus. This raises the more general question whether MED25 has dual functions in recruiting both histone acetylases and histone deacetylases for the transcription factors to which MED25 can bind? (e.g. in the context of ARF targets, PIF4/BZR1 targets and MYC2 targets)?

\section{A putative model for the role of MED25 in regulating YUCCA8 expression.}

Our results and those by van der Woude (2018) combined now provide the basis for an updated model for transcription at the YUCCA8 locus induced by warmth: at normal temperature the YUC8 promoter is mostly closed due to packaging in H2A.Z type histones. Both PIF4 and BZR1 may be bound infrequent to the YUCCA8 promoter and potential recruitment of HDA9 to the YUC8 promoter by binding to PIF4 is inefficient due to high turnover of HDA9 at ambient temperature. Upon warmth also HDA9 protein is stabilised and is now more efficiently recruited to the YUC8 promoter by binding to MED25, possibly replacing bound PIF4. At the YUC8 promoter HDA9 causes the deacetylation of histones near 
the transcription start, which leads to eviction of repressing H2A.Z histones (van der Woude et al., 2018 under review). Subsequently, the interaction between MED25 and HDA9 results in a destruction of HDA9 protein, as demonstrated by the increased HDA9 stability in pft1-2 and decreased stability of HDA9 in MED24OE. The removal of HDA9 from the GD-domain of MED25 allows subsequently interaction with PIF4 protein, which is now more abundant due to increased transcriptional activity of BZR1 (Ibañez et al., 2018). Future research will need to determine the role of histone acetylases in the regulation of YUCCA8 and whether MED25 plays a role in this as well.

\section{Reference}

An C, Li L, Zhai Q, You Y, Deng L, Wu F, Chen R, Jiang H, Wang H, Chen Q, Li C (2017) Mediator subunit MED25 links the jasmonate receptor to transcriptionally active chromatin. Proceedings of the National Academy of Sciences

Backstrom S, Elfving N, Nilsson R, Wingsle G, Bjorklund S (2007) Purification of a plant mediator from Arabidopsis thaliana identifies PFT1 as the Med25 subunit. Mol Cell 26: 717-729

Black JC, Choi JE, Lombardo SR, Carey M (2006) A mechanism for coordinating chromatin modification and preinitiation complex assembly. Mol Cell 23: 809-818

Bours R, Kohlen W, Bouwmeester HJ, van der Krol A (2015) Thermoperiodic control of hypocotyl elongation depends on auxin-induced ethylene signaling that controls downstream PHYTOCHROME INTERACTING FACTOR3 activity. Plant Physiol 167: 517530

Cerdan PD, Chory J (2003) Regulation of flowering time by light quality. 423: 881-885

Cevik V, Kidd BN, Zhang P, Hill C, Kiddle S, Denby KJ, Holub EB, Cahill DM, Manners JM, Schenk PM, Beynon J, Kazan K (2012) MEDIATOR25 acts as an integrative hub for the regulation of jasmonate-responsive gene expression in Arabidopsis. Plant Physiol 160: 541-555

Chen H, Zou Y, Shang Y, Lin H, Wang Y, Cai R, Tang X, Zhou J-M (2008) Firefly Luciferase Complementation Imaging Assay for Protein-Protein Interactions in Plants. Plant Physiology 146: 368

Chen R, Jiang H, Li L, Zhai Q, Qi L, Zhou W, Liu X, Li H, Zheng W, Sun J, Li C (2012) The Arabidopsis mediator subunit MED25 differentially regulates jasmonate and abscisic acid signaling through interacting with the MYC2 and ABI5 transcription factors. Plant Cell 24: 2898-2916

Clough SJ, Bent AF (1998) Floral dip: a simplified method for Agrobacterium-mediated transformation of Arabidopsis thaliana. Plant J 16

Coleman-Derr D, Zilberman D (2012) Deposition of Histone Variant H2A.Z within Gene Bodies Regulates Responsive Genes. PLOS Genetics 8: e1002988 
Cortijo S, Charoensawan V, Brestovitsky A, Buning R, Ravarani C, Rhodes D, van Noort J, Jaeger KE, Wigge PA (2017) Transcriptional Regulation of the Ambient Temperature Response by H2A.Z Nucleosomes and HSF1 Transcription Factors in Arabidopsis. Mol Plant 10: 1258-1273

Delker C, Sonntag L, James GV, Janitza P, Ibanez C, Ziermann H, Peterson T, Denk K, Mull S, Ziegler J, Davis SJ, Schneeberger K, Quint M (2014) The DET1-COP1-HY5 pathway constitutes a multipurpose signaling module regulating plant photomorphogenesis and thermomorphogenesis. Cell Rep 9: 1983-1989

Devoto A, Nieto-Rostro M, Xie D, Ellis C, Harmston R, Patrick E, Davis J, Sherratt L, Coleman M, Turner JG (2002) COI1 links jasmonate signalling and fertility to the SCF ubiquitinligase complex in Arabidopsis. Plant J 32: 457-466

Elfving N, Davoine C, Benlloch R, Blomberg J, Brannstrom K, Muller D, Nilsson A, Ulfstedt M, Ronne H, Wingsle G, Nilsson O, Bjorklund S (2011) The Arabidopsis thaliana Med25 mediator subunit integrates environmental cues to control plant development. Proc Natl Acad Sci U S A 108: 8245-8250

Englert NA, Luo G, Goldstein JA, Surapureddi S (2015) Epigenetic modification of histone 3 lysine 27: mediator subunit MED25 is required for the dissociation of polycomb repressive complex 2 from the promoter of cytochrome P450 2C9. J Biol Chem 290: 2264-2278

Franklin KA, Lee SH, Patel D, Kumar SV, Spartz AK, Gu C, Ye S, Yu P, Breen G, Cohen JD, Wigge PA, Gray WM (2011) Phytochrome-interacting factor 4 (PIF4) regulates auxin biosynthesis at high temperature. Proc Natl Acad Sci U S A 108: 20231-20235

Gonzalez D, Bowen AJ, Carroll TS, Conlan RS (2007) The transcription corepressor LEUNIG interacts with the histone deacetylase HDA19 and mediator components MED14 (SWP) and CDK8 (HEN3) to repress transcription. Mol Cell Biol 27: 5306-5315

Gray WM, Ostin A, Sandberg G, Romano CP, Estelle M (1998) High temperature promotes auxin-mediated hypocotyl elongation in Arabidopsis. Proceedings of the National Academy of Sciences of the United States of America 95: 7197-7202

Hornitschek P, Kohnen MV, Lorrain S, Rougemont J, Ljung K, Lopez-Vidriero I, Franco-Zorrilla JM, Solano R, Trevisan M, Pradervand S, Xenarios I, Fankhauser C (2012) Phytochrome interacting factors 4 and 5 control seedling growth in changing light conditions by directly controlling auxin signaling. Plant J 71: 699-711

Huq E, Quail PH (2002) PIF4, a phytochrome-interacting bHLH factor, functions as a negative regulator of phytochrome $B$ signaling in Arabidopsis. EMBO J 21: 2441-2450

Ibañez C, Delker C, Martinez C, Bürstenbinder K, Janitza P, Lippmann R, Ludwig W, Sun H, James GV, Klecker M, Grossjohann A, Schneeberger K, Prat S, Quint M (2018) Brassinosteroids Dominate Hormonal Regulation of Plant Thermomorphogenesis via BZR1. Current Biology 28: 303-310.e303

Inigo S, Alvarez MJ, Strasser B, Califano A, Cerdan PD (2012) PFT1, the MED25 subunit of the plant Mediator complex, promotes flowering through CONSTANS dependent and independent mechanisms in Arabidopsis. Plant J 69: 601-612

Ito J, Fukaki H, Onoda M, Li L, Li C, Tasaka M, Furutani M (2016) Auxin-dependent compositional change in Mediator in ARF7- and ARF19-mediated transcription. Proc Natl Acad Sci U S A 113: 6562-6567

Kazan K (2017) The Multitalented MEDIATOR25. Front Plant Sci 8: 999 
Kidd BN, Aitken EA, Schenk PM, Manners JM, Kazan K (2010) Plant mediator: mediating the jasmonate response. Plant Signal Behav 5: 718-720

Kidd BN, Cahill DM, Manners JM, Schenk PM, Kazan K (2011) Diverse roles of the Mediator complex in plants. Semin Cell Dev Biol 22: 741-748

Kidd BN, Edgar Cl, Kumar KK, Aitken EA, Schenk PM, Manners JM, Kazan K (2009) The mediator complex subunit PFT1 is a key regulator of jasmonate-dependent defense in Arabidopsis. Plant Cell 21: 2237-2252

Kim K, Jeong J, Kim J, Lee N, Kim ME, Lee S, Chang Kim S, Choi G (2016) PIF1 Regulates Plastid Development by Repressing Photosynthetic Genes in the Endodermis. Mol Plant 9: 1415-1427

Klose C, Buche C, Fernandez AP, Schafer E, Zwick E, Kretsch T (2012) The mediator complex subunit PFT1 interferes with COP1 and HY5 in the regulation of Arabidopsis light signaling. Plant Physiol 160: 289-307

Koini MA, Alvey L, Allen T, Tilley CA, Harberd NP, Whitelam GC, Franklin KA (2009) High temperature-mediated adaptations in plant architecture require the bHLH transcription factor PIF4. Curr Biol 19: 408-413

Kumar SV, Lucyshyn D, Jaeger KE, Alos E, Alvey E, Harberd NP, Wigge PA (2012) Transcription factor PIF4 controls the thermosensory activation of flowering. Nature 484: 242-245

Kumar SV, Wigge PA (2010) H2A.Z-containing nucleosomes mediate the thermosensory response in Arabidopsis. Cell 140: 136-147

Lee J, He K, Stolc V, Lee H, Figueroa P, Gao Y, Tongprasit W, Zhao H, Lee I, Deng XW (2007) Analysis of transcription factor HY5 genomic binding sites revealed its hierarchical role in light regulation of development. Plant Cell 19: 731-749

Leivar P, Monte E, Al-Sady B, Carle C, Storer A, Alonso JM, Ecker JR, Quail PH (2008) The Arabidopsis phytochrome-interacting factor PIF7, together with PIF3 and PIF4, regulates responses to prolonged red light by modulating phyB levels. Plant Cell 20: 337-352

Liu J, Cheng X, Liu P, Li D, Chen T, Gu X, Sun J (2017) MicroRNA319-regulated TCPs interact with FBHs and PFT1 to activate CO transcription and control flowering time in Arabidopsis. PLoS Genet 13: e1006833

Malik S, Roeder RG (2010) The metazoan Mediator co-activator complex as an integrative hub for transcriptional regulation. Nat Rev Genet 11: 761-772

Martínez C, Espinosa-Ruíz A, de Lucas M, Bernardo-García S, Franco-Zorrilla JM, Prat S (2018) PIF4-induced BR synthesis is critical to diurnal and thermomorphogenic growth. The EMBO Journal

Mockler TC, Michael TP, Priest HD, Shen R, Sullivan CM, Givan SA, McEntee C, Kay SA, Chory J (2007) The DIURNAL project: DIURNAL and circadian expression profiling, modelbased pattern matching, and promoter analysis. Cold Spring Harb Symp Quant Biol 72: 353-363

Nawkar GM, Kang CH, Maibam P, Park JH, Jung YJ, Chae HB, Chi YH, Jung IJ, Kim WY, Yun DJ, Lee SY (2017) HY5, a positive regulator of light signaling, negatively controls the unfolded protein response in \&lt;em\&gt;Arabidopsis\&lt;/em\&gt. Proceedings of the National Academy of Sciences 114: 2084

Oh E, Zhu J-Y, Bai M-Y, Arenhart RA, Sun Y, Wang Z-Y (2014) Cell elongation is regulated through a central circuit of interacting transcription factors in the Arabidopsis hypocotyl. eLife 3: e03031 
Oh E, Zhu JY, Wang ZY (2012) Interaction between BZR1 and PIF4 integrates brassinosteroid and environmental responses. Nat Cell Biol 14: 802-809

Ou B, Yin KQ, Liu SN, Yang Y, Gu T, Wing Hui JM, Zhang L, Miao J, Kondou Y, Matsui M, Gu HY, Qu U (2011) A high-throughput screening system for Arabidopsis transcription factors and its application to Med25-dependent transcriptional regulation. Mol Plant 4: 546-555

Park E, Park J, Kim J, Nagatani A, Lagarias JC, Choi G (2012) Phytochrome B inhibits binding of phytochrome-interacting factors to their target promoters. Plant J 72: 537-546

Pfeiffer A, Shi H, Tepperman JM, Zhang Y, Quail PH (2014) Combinatorial complexity in a transcriptionally centered signaling hub in Arabidopsis. Mol Plant 7: 1598-1618

Quint M, Delker C, Franklin KA, Wigge PA, Halliday KJ, van Zanten M (2016) Molecular and genetic control of plant thermomorphogenesis. Nat Plants 2: 15190

Rival P, Press MO, Bale J, Grancharova T, Undurraga SF, Queitsch C (2014) The conserved PFT1 tandem repeat is crucial for proper flowering in Arabidopsis thaliana. Genetics 198: $747-754$

Samanta S, Thakur JK (2015) Importance of Mediator complex in the regulation and integration of diverse signaling pathways in plants. Frontiers in Plant Science 6: 757

Saxena P, Hsieh YC, Alvarado VY, Sainsbury F, Saunders K, Lomonossoff GP, Scholthof HB (2011) Improved foreign gene expression in plants using a virus-encoded suppressor of RNA silencing modified to be developmentally harmless. Plant Biotechnol J 9: 703712

Seguela-Arnaud M, Smith C, Uribe MC, May S, Fischl H, McKenzie N, Bevan MW (2015) The Mediator complex subunits MED25/PFT1 and MED8 are required for transcriptional responses to changes in cell wall arabinose composition and glucose treatment in Arabidopsis thaliana. BMC Plant Biol 15: 215

Sharma D, Fondell JD (2002) Ordered recruitment of histone acetyltransferases and the TRAP/Mediator complex to thyroid hormone-responsive promoters in vivo. Proc Natl Acad Sci U S A 99: 7934-7939

Soutourina J (2018) Transcription regulation by the Mediator complex. Nat Rev Mol Cell Biol 19: $262-274$

Sun J, Qi L, Li Y, Chu J, Li C (2012) PIF4-mediated activation of YUCCA8 expression integrates temperature into the auxin pathway in regulating arabidopsis hypocotyl growth. PLoS Genet 8: e1002594

Sun J, Qi L, Li Y, Chu J, Li C (2012) PIF4-Mediated Activation of YUCCA8 Expression Integrates Temperature into the Auxin Pathway in Regulating Arabidopsis Hypocotyl Growth. PLOS Genetics 8: e1002594

Sun J, Qi L, Li Y, Zhai Q, Li C (2013) PIF4 and PIF5 transcription factors link blue light and auxin to regulate the phototropic response in Arabidopsis. Plant Cell 25: 2102-2114

Sura W, Kabza M, Karlowski WM, Bieluszewski T, Kus-Slowinska M, Pawełoszek $\measuredangle$, Sadowski J, Ziolkowski PA (2017) Dual Role of the Histone Variant H2A.Z in Transcriptional Regulation of Stress-Response Genes. The Plant Cell 29: 791

Tasset C, Singh Yadav A, Sureshkumar S, Singh R, van der Woude L, Nekrasov M, Tremethick D, van Zanten M, Balasubramanian S (2018) POWERDRESS-mediated histone deacetylation is essential for thermomorphogenesis in Arabidopsis thaliana. PLOS Genetics 14: e1007280 
Xu R, Li Y (2011) Control of final organ size by Mediator complex subunit 25 in Arabidopsis thaliana. Development 138: 4545-4554

Yang Y, Ou B, Zhang J, Si W, Gu H, Qin G, Qu LJ (2014) The Arabidopsis Mediator subunit MED16 regulates iron homeostasis by associating with EIN3/EIL1 through subunit MED25. Plant J 77: 838-851

Yuan L, Chen X, Chen H, Wu K, Huang S (2018) Histone deacetylases HDA6 and HDA9 coordinately regulate valve cell elongation through affecting auxin signaling in Arabidopsis. Biochemical and Biophysical Research Communications

Zhang B, Holmlund M, Lorrain S, Norberg M, Bako L, Fankhauser C, Nilsson O (2017) BLADEON-PETIOLE proteins act in an E3 ubiquitin ligase complex to regulate PHYTOCHROME INTERACTING FACTOR 4 abundance. Elife 6

Zhang F, Yao J, Ke J, Zhang L, Lam VQ, Xin XF, Zhou XE, Chen J, Brunzelle J, Griffin PR, Zhou M, Xu HE, Melcher K, He SY (2015) Structural basis of JAZ repression of MYC transcription factors in jasmonate signalling. Nature 525: 269-273

Zhang X, Henriques R, Lin S-S, Niu Q-W, Chua N-H (2006) Agrobacterium-mediated transformation of Arabidopsis thaliana using the floral dip method. 1: 641-646

Zhu Y, Schluttenhoffer CM, Wang P, Fu F, Thimmapuram J, Zhu JK, Lee SY, Yun DJ, Mengiste T (2014) CYCLIN-DEPENDENT KINASE8 differentially regulates plant immunity to fungal pathogens through kinase-dependent and -independent functions in Arabidopsis. Plant Cell 26: 4149-4170 


\section{Supplementary Files}

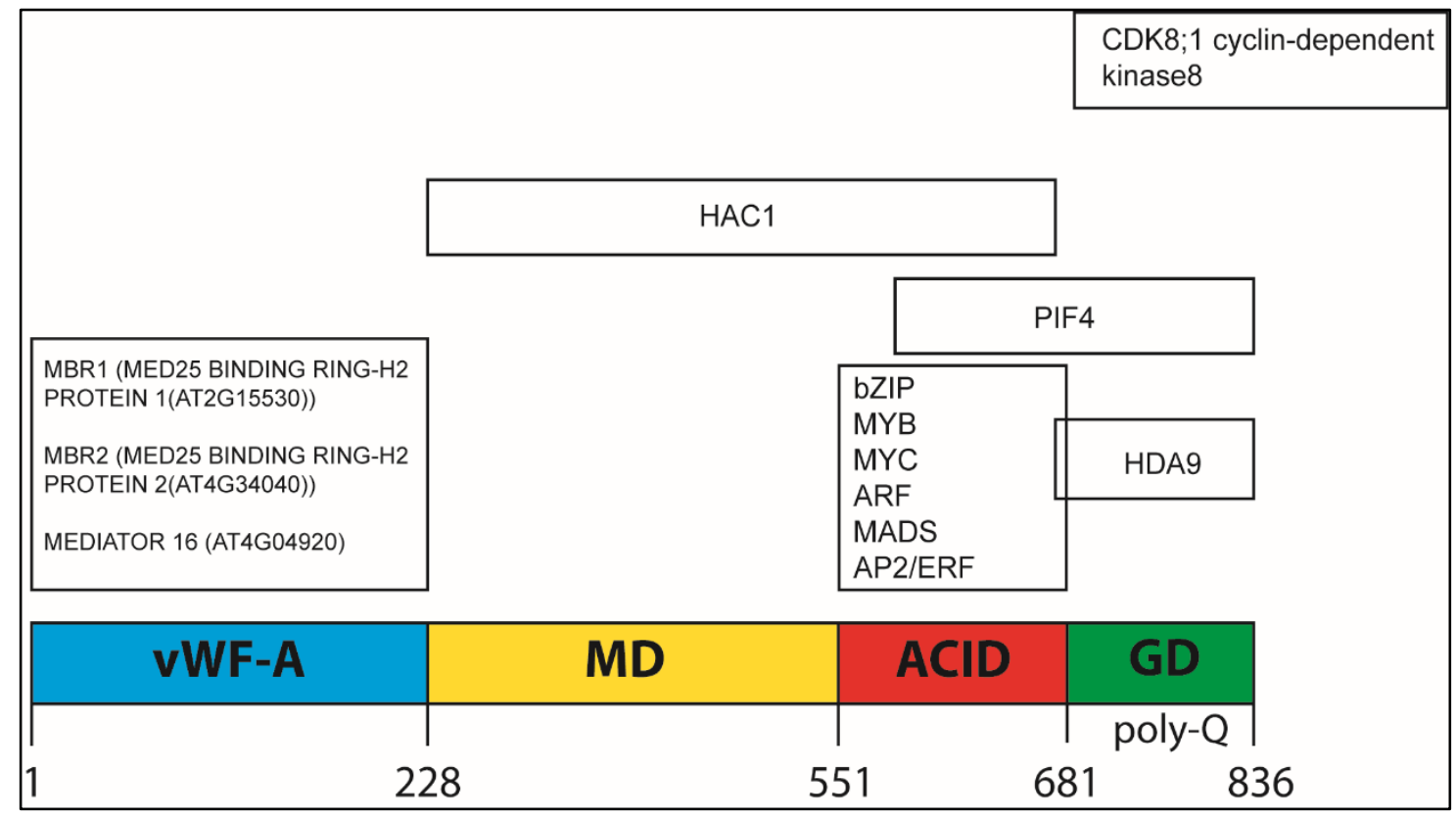

Figure S1. Interaction of different proteins with sub-domains of MED25. 

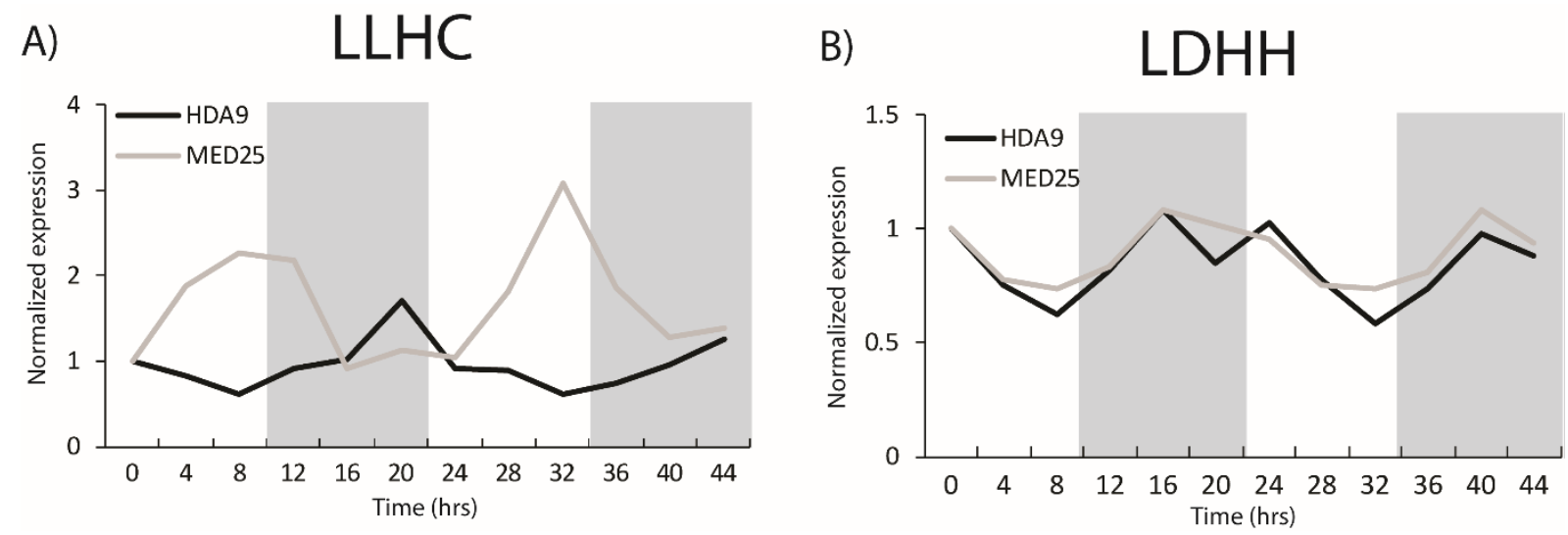

Figure S2. MED25 and HDA9 diurnal expression profiles under LLHC and LDHH. The expression pattern of HDA9 and MED25 genes was retrieved from Diurnal database from Mockler Laboratory database (http://www.diurnal.cgrb.oregonstate.edu). The normalized data were profiled under (A) diurnal temperature cycles LLHC $\left(31^{\circ} \mathrm{C}\right.$, day, $20^{\circ} \mathrm{C}$, night) or (B) diurnal light cycles at high temperature $\left(31^{\circ} \mathrm{C} ; \mathrm{HH}\right)$ 


\begin{tabular}{|c|c|c|c|c|c|c|}
\hline \# & Gene & ID acc. & TF family & Responses & Methods & Ref \\
\hline 1 & DREB2A & AT5G05410 & AP2; ERF & drought & $\mathrm{Y} 2 \mathrm{H}$ & $\begin{array}{l}\text { (Elfving et al., 2011; } \\
\text { Cevik et al., 2012) }\end{array}$ \\
\hline 2 & RAP2.2 & AT3G14230 & AP2; ERF & ethylene & $\mathrm{Y} 2 \mathrm{H}$ & (Ou et al., 2011) \\
\hline 3 & ERF95 & AT3G23220 & AP2; ERF & ethylene & $\mathrm{Y} 2 \mathrm{H}$ & (Ou et al., 2011) \\
\hline 4 & TDR1 & AT3G23230 & AP2; ERF & ethylene & $\mathrm{Y} 2 \mathrm{H}$ & $\begin{array}{l}\text { (Ou et al., 2011; } \\
\text { Cevik et al., 2012) }\end{array}$ \\
\hline 5 & ERF1 & AT3G23240 & AP2; ERF & ethylene & $\mathrm{Y} 2 \mathrm{H}$ & $\begin{array}{l}\text { (Ou et al., 2011; } \\
\text { Cevik et al., 2012) }\end{array}$ \\
\hline 6 & - & AT4G18450 & AP2; ERF & ethylene & $\mathrm{Y} 2 \mathrm{H}$ & (Ou et al., 2011) \\
\hline 7 & ERF109 & AT4G34410 & AP2; ERF & ethylene & $\mathrm{Y} 2 \mathrm{H}$ & (Ou et al., 2011) \\
\hline 8 & ORA59 & AT1G06160 & AP2; ERF & ethylene & $\mathrm{Y} 2 \mathrm{H}$ & (Cevik et al., 2012) \\
\hline 9 & ERF15 & AT2G31230 & AP2; ERF & ethylene & $\mathrm{Y} 2 \mathrm{H}$ & (Cevik et al., 2012) \\
\hline 10 & WIN1 & AT1G15360 & AP2; ERF & ethylene & - & (Zhu et al., 2014) \\
\hline 11 & EIN3 & AT3G20770 & EIN3; EIL & ethylene & $\begin{array}{l}\text { Y2H, BiFC, } \\
\text { Split LUC } \\
\text { Y2H,BiFC, }\end{array}$ & (Yang et al., 2014) \\
\hline 12 & EIL1 & AT2G27050 & EIN3; EIL & ethylene & Split LUC & (Yang et al., 2014) \\
\hline 13 & BZS1 & AT4G39070 & DBB & $\begin{array}{l}\text { BR } \\
\text { signalling }\end{array}$ & $\mathrm{Y} 2 \mathrm{H}$ & $\begin{array}{l}\text { (Ou et al., 2011; } \\
\text { Cevik et al., 2012) }\end{array}$ \\
\hline 14 & WRKY10 & AT1G55600 & WRKY & - & $\mathrm{Y} 2 \mathrm{H}$ & (Cevik et al., 2012) \\
\hline 15 & MYB104 & AT2G26950 & MYB & - & $\mathrm{Y} 2 \mathrm{H}$ & (Cevik et al., 2012) \\
\hline 16 & ZFHD1 & AT1G69600 & ZF-HD & $\begin{array}{l}\text { Salt, } \\
\text { drought, } \\
\text { ABA }\end{array}$ & $\mathrm{Y} 2 \mathrm{H}$ & (Elfving et al., 2011) \\
\hline 17 & POSF21 & AT2G31370 & bZIP & Salt stress & $\mathrm{Y} 2 \mathrm{H}$ & (Cevik et al., 2012) \\
\hline 18 & PHL1 & AT5G29000 & Myb/SANT & P starvation & $\mathrm{Y} 2 \mathrm{H}$ & $\begin{array}{l}\text { (Elfving et al., 2011; } \\
\text { Ou et al., 2011) }\end{array}$ \\
\hline 19 & MYC2 & AT1G32640 & bHLH & JA signaling & $\mathrm{Y} 2 \mathrm{H}$ & (Cevik et al., 2012) \\
\hline 20 & MYC3 & AT5G46760 & bHLH & JA signaling & IP & (Zhang et al., 2015) \\
\hline 21 & MYC4 & AT4G17880 & bHLH & JA signaling & $\mathrm{Y} 2 \mathrm{H}$ & (Cevik et al., 2012) \\
\hline 22 & ABI5 & AT2G36270 & bZIP & $\begin{array}{l}\text { ABA } \\
\text { signaling }\end{array}$ & $\mathrm{BiFC}$ & (Chen et al., 2012) \\
\hline 23 & ARF7 & AT5G20730 & B3; ARF & $\begin{array}{l}\text { Auxin } \\
\text { signaling }\end{array}$ & $\mathrm{Y} 2 \mathrm{H}$ & (Ito et al., 2016) \\
\hline
\end{tabular}




\begin{tabular}{l|llllll}
24 & ARF19 & AT1G19220 & B3; ARF & $\begin{array}{l}\text { Auxin } \\
\text { signaling }\end{array}$ & Y2H & (Ito et al., 2016) \\
25 & TCP4 & AT3G15030 & TCP & $\begin{array}{l}\text { Flowering } \\
\text { time }\end{array}$ & Split LUC & (Liu et al., 2017) \\
26 & FBH1 & AT1G35460 & bHLH & $\begin{array}{l}\text { Flowering } \\
\text { time }\end{array}$ & Split LUC & (Liu et al., 2017) \\
27 & PIF4 & AT2G43010 & bHLH & Growth & Split LUC & In study \\
& & & & BR & Split LUC & In study \\
\hline
\end{tabular}

Table-S1. List of Transcription factors that physically interact with the MED25. 


\begin{tabular}{|c|c|c|c|}
\hline Primer name & Sequence & Used for & Ref. \\
\hline pft1-2-F & TGGAACTGGTCCAACAGAAC & Mutant screening & this study \\
\hline pft1-2-R & TGCATTGGCTTTCTTCCATAC & Mutant screening & this study \\
\hline Salk LBb1.3 & ATTTTGGCGATTTCGGAAC & Mutant screening & this study \\
\hline PIF4 CDS F & ATGGAACACCAAGGTTGGAG & LCl construct & this study \\
\hline PIF4 CDS R & GTGGTCCAAACGAGAACCGT & LCl construct & this study \\
\hline HDA9 CDS F & ATGCGTTCCAAGGACAAAAT & LCl construct & this study \\
\hline HDA9 CDS R & TGACGCATCGTTATCGTTGT & $\mathrm{LCl}$ construct & this study \\
\hline MED25 CDS F & ATGTCGTCGGAGGTGAAACA & $\mathrm{LCl}$ construct & this study \\
\hline MED25 CDS R & TCCCATGAAGCCAGCTCC & $\mathrm{LCl}$ construct & this study \\
\hline MED25 ${ }^{\text {VWF-A_nLUC_F }}$ & ATGTCGTCGGAGGTGAAACA & $\mathrm{LCl}$ construct & this study \\
\hline MED25 ${ }^{\text {VWF-A_nLUC_R }}$ & CTCCGAGATCAGGACAAGATAGA & $\mathrm{LCl}$ construct & this study \\
\hline MED25 ${ }^{M D}$-nLUC_F & ATGAATTTTGTGGAGGCATGTGC & $\mathrm{LCl}$ construct & this study \\
\hline MED25 ${ }^{M D}$-nLUC_R & CTGCATAGCCCCCGATG & $\mathrm{LCl}$ construct & this study \\
\hline MED25 $5^{A C I D}$-nLUC_F & ATGACTTCACAATCCAAATATGTGAA & LCl construct & this study \\
\hline MED25ACID-nLUC_R & ATTTGGAATTTGTGGTTTAAACA & $\mathrm{LCl}$ construct & this study \\
\hline MED25 $5^{G D}-n L U C \_F$ & ATGCAGCAACAGCAGCAGCAACAACAA & $\mathrm{LCl}$ construct & this study \\
\hline MED25 ${ }^{\text {GD-nLUC_R }}$ & TCCCATGAAGCCAGCTCC & $\mathrm{LCl}$ construct & this study \\
\hline qPIF4-F & ACTCAGATGCAGCCGATGG & qPCR & this study \\
\hline qPIF4-R & ACGTAATGAAGTTGCACGTTT & qPCR & this study \\
\hline qYUCCA8-F & TTTTCTCCCGTAGCCACCAC & qPCR & this study \\
\hline qYUCCA8-R & CGATGAGACCAGTGGCTTGT & qPCR & this study \\
\hline
\end{tabular}

Table S2. The list of primers were used in this work. 



\title{
CHAPTER-5
}

Functional intron-derived miRNAs and host-gene expression in plants

\author{
Umidjon Shapulatov \\ Mark van Hoogdalem
}

Marielle Schreuder

Harro Bouwmeester

Ibrokhim Y. Abdurakhmonov

Alexander R. van der Krol

Published in Plant Methods (2018) 14:83

https://doi.org/10.1186/s13007-018-0351-2 


\begin{abstract}
Background

Recently, putative pre-miRNAs locations have been identified in the introns of plant genes, raising the question whether such genes can show a dual functionality by having both correct maturation of the host gene pre-mRNA and maturation of the miRNAs from the intron. Here, we demonstrated that such dual functionality is indeed possible, using as host gene the firefly luciferase gene with intron (ffgLUC), and different artificial intronic miRNAs (aimiRNA) placed within the intron of ffgLUC.
\end{abstract}

\title{
Results
}

The miRNAs were based on the structure of the natural miR319a. Luciferase (LUC) activity in planta was used to evaluate a correct splicing of the ffgLUC mRNA. Different target sequences were inserted into the aimiRNA to monitor efficiency of silencing of different target mRNAs. After adjusting the insertion cloning strategy, the ffgLUC ${ }^{\text {aimiR-319a }}$ gene showed dual functionality with correct splicing of ffgLUC and efficient silencing of TEOSINTE BRANCHED1/CYCLOIDEA/PROLIFERATING CELL FACTOR1 (TCP) transcription factor genes targeted in-trans by aimiR-319a or targeting the transgene ffLUC in-cis by an aimiR-LUC. Silencing of endogenous target genes by aimiRNA or amiRNA is efficient both in transient assays and stable transformants. A behave as strong phenotype the PHYTOCHROME B (PHYB) gene was also targeted by ffgLUC aimiR-PHYB. The lack of silencing of the PHYB target was most likely due to an insensitive target site within the PHYB mRNA which can potentially form a double stranded stem structure.

\section{Conclusion}

The combination of an overexpression construct with an artificial intronic microRNA allows for a simultaneous dual function in plants. The concept therefore adds new options to engineering of plant traits that require multiple gene manipulations.

Keywords: Intron, imiRNA, aimiRNA, miRNA, amiRNA, luciferase 


\section{Background}

Important traits of crop plants have successfully been manipulated by selection of mutants (Peng et al., 1999), by ectopic expression of a transgene (Kasuga et al., 1999; Karaba et al., 2007; Chen et al., 2016), or by silencing of a single gene (Schwab et al., 2006; Park et al., 2009). However, because of the complexity of gene-networks in plants, the effect of many singlegene disturbances is limited due to buffering capacity of such networks (Prelich, 2012; Watson et al., 2013). Moreover, plant trait manipulation may potentially benefit from synergistic interaction between independent transgene manipulations. Stacking of independent transgenes is time-consuming, especially in crops that are difficult targets for transformation. Engineering in recalcitrant crops may therefore benefit from techniques that can target multiple genes by a single transformation event.

MicroRNAs (miRNA) are short (19-22nt) non coding RNAs that can silence the expression of specific target genes and natural miRNAs form an integral part of developmental decisions in plants (Reinhart et al., 2002; Bartel, 2004). From all plant miRNAs listed in the microRNA database ( http://www.mirbase.org/ ) only a small number have been functionally characterised. Moreover, while most miRNA are processed from regular non-coding miRNAgenes, recently, protein-coding genes with introns containing potential miRNA sequences have been identified both in mammals and plants. For instance, the Arabidopsis thaliana genome contains 37 protein coding genes with intronic miRNAs (imiRNAs) and the rice genome contains 181 protein coding genes with imiRNAs (Yang et al., 2012). At present, there is no experimental evidence that plant genes containing imiRNAs show simultaneous dual functionality: a correct intron splicing of the host gene pre-mRNA to form a mRNA encoding a functional protein and processing of the miRNA from the intron for effective silencing of the target gene. For instance, in some cases the miRNA encoded in the intron is only produced as alternatively spliced transcript (Yan et al., 2012). In such cases, correct mRNA splicing and gene expression and miRNA production from the intron may be mutually exclusive. Functionality of intron-derived miRNAs has been demonstrated in mammals, C. elegans, zebra fish, and chicken (Lin et al., 2006). It has been demonstrated that an imiRNA can be correctly processed from the intron sequence, without interfering with the accuracy of the splicing process of the host gene (Parsi et al., 2012; Kashyap et al., 2013). The intron-derived miRNAs require type-II 
RNA polymerases (Pol-II) and spliceosome components for their biogenesis. In animals, it has been shown that regular miRNA processing is dependent on Drosha-mediated cleavage, but initial processing of some imiRNAs are Drosha-independent. Instead, initial imiRNA processing is coupled to the intron splicing reaction (Ruby et al., 2007). How imiRNAs are processed in plants is not fully known at present. Introns and active 5' splice sites (5'ss) have been shown to stimulate the accumulation of miRNAs encoded within the first exons of intron-containing MIR genes and Knop et al found that the $5^{\prime}$-splice site is crucial for the regulation of intronic miRNA-402 biogenesis from the first intron of host gene At1g77230 (Knop et al., 2017). Moreover, the gene encoding dicer protein DCL1 contains imiR838 in intron 14 and the gene can produce both functional DCL1 mRNA and mature miR838. In this instance the imiRNA biogenesis and DCL1 mRNA biogenesis are mutually exclusive but in a population both gene products may be produced (Ren and Yu, 2012).

Here, we tested whether a protein coding transgene can be effectively expressed in plants, while also producing a functional miRNA. The feasibility and requirements for such dual gene functionality were determined using three gene construct (ffgLUC ${ }^{\text {aimiR-319a }}$, ffgLUC ${ }^{\text {aimiR-LUC }}$ and ffgLUC CimiR-PHYB), designed to report on both protein and miRNA function. For overexpression, the firefly Luciferase gene with a single intron (ffgLUC) was used, which allows for easy monitoring of gene activity and splicing accuracy. As template for the miRNA sequence in the intron, the sequence of the natural ath-miR319a was used (Nag et al., 2009). For alternative targets, the 21-bp sequence targeting TEOSINTE BRANCHED1/CYCLOIDEA/PROLIFERATING CELL FACTOR1 (TCP) transcription factor in miR319a was replaced by a 21 nucleotide sequence targeting ffgLUC mRNA (Liang et al., 2012) or 21-bp targeting the Arabidopsis thaliana PHYB mRNA (AT2G18790). Initially, insertion of the miRNA into the LUC intron resulted in a loss of LUC activity, indicating incorrect splicing of the intron from the LUC pre-mRNA. However, after adjusting the miRNA position within the intron, the transgene showed normal LUC activity when expressed in plants, indicating accurate splicing of the LUC pre-mRNA. Moreover, the aimiRNA targeting TCPs or ffgLUC both were able to suppress target gene expression, indicating effective processing of the aimiRNA from the ffgLUC intron. The concept of a transgene containing an aimiRNA could be useful for simultaneous manipulation of several gene activities, which could be an important tool for plant biotechnology. 


\section{Methods}

Plant materials and growth condition. Arabidopsis thaliana (Col-0 background, N1092) was used for stable transformation. The Arabidopsis phyB-9 T-DNA insertion mutant (\#CS6217) was obtained from the NASC stock collection. Plants were grown on rock-wool in a growth chamber at $12 \mathrm{hL} / 12 \mathrm{hD}$ at $22^{\circ} \mathrm{C}$ on half strength Hoagland-nutrient solution.

Cloning of expression constructs. Artificial microRNAs constructs were created using athmiR319a backbone as described by Liang (Liang et al., 2012). The primer sequences used are listed in Table S2. The artificial miRNA nucleotide sequences 5'-TAACTGTAAACCGAAAGGCTG3' for the AthPHYB (AT2G18790) were selected using WMD3-Web MicroRNA Designer (http://wmd3.weigelworld.org/cgi-bin/webapp.cgi). The IDT RNAi design tools (Integrated DNA Technologies) was used to design the amiRNA nucleotide sequence targeting the luciferase mRNA (5'-TAGAACTGCCTGCGTCAGATT-3'). Pre-microRNA 319a was amplified directly from $A$. thaliana genomic DNA using primers (CAAACACACGCTCGGACGCAT-F and CATGGCGATGCCTTAAATAAAG-R). The aimiRNA sequences were amplified from premiRNA319a using specific primers which added EcoR V and EcoR I restriction sites for cloning into the intron of ffgLUC (GATATCAGAGAGCTTCCTTGAGTCCATTCAC-F and GAATTCAGGGAGCTCCCTTCAGTCCAATC-R). For amplification of the aimiR-LUC the TCP target sequence in the primers was replaced by the selected LUC target sequence (GATATCTATAACTGCCTGCCTCAGATAAGGTCGTGATATGATTCA-F GAATTCTAGAACTGCCTGCGTCAGATTAAAGAGAATCAATGATCCA-R). For the amplification of the aimiR-PHYB the TCP target sequence in the primers was replaced by the selected PHYB target sequence (GATATCTAGCTGTAAACCGTAAGGCTCAGGTCGTGATATGATTCA-F and GAATTCTAACTGTAAACCGAAAGGCTGAAAGAGAATCAATGATCCA-R). To generate of ffgLUC del $^{\text {aimiR319a }}$ construct the first exon plus 10 nucleotides from $5^{\prime}$ site of intron was amplified by using primer which introduce Nco $\mathrm{I}$ at start codon and EcoRV site in intron (CCATGGAAGACGCCAAAAAC-F and GATATCAGAAACTTACGTAATGTTCACCTCG-R). The second exon plus 61 base pair from 3' site of the intron was amplified using primers which introduce an EcoR I site at the end of the intron sequence and an Not I site after the stop codon (GAATTCAACTTTTCTAATATATGACCAAAATTTGTT-F and GCGGCCGCTTACAATTTGGACTTTCCGCCCTT-R). To generate of $\mathrm{ffgLUC}^{\text {imiR319a }} \mathrm{ffgLUC}^{\text {aimiR-LUC }}$ and 
ffgLUC CamiR-PHYB constructs, the first exon plus 33 nucleotides from the $5^{\prime}$ end of the intron was amplified by using primer pairs introducing an Nco I at the ATG start codon and an EcoR V site at the end of the intron sequence (CCATGGAAGACGCCAAAAAC-F and GATATCTACTAATTAATGATAATTATT-R). The second exon of ffgLUC was amplified from 135 base pairs from 3 ' splice site to after the stop codon, introducing an EcoR I site at in the intron and Not I site after the stop codon, using the primer pairs (GAATTCGTAATATAATATTTCAAATATTTTTTTCAAAATAA-F GCGGCCGCTTACAATTTGGACTTTCCGCCCTT-R). The resulting PCR products were digested with EcoR I and EcoR V and the amiRNAs product was ligated into the ffgLUC intron. The ffgLUC was amplified with primers introducing an Nco I site at the ATG and Not I site after the stop codon (CCATGGAAGACGCCAAAAAC-F and CGGCCGCTTACAATTTGGACTTTCCGCCCTT-R). The ffgLUC, ffgLUCimiR319a, ffgLUC ${ }^{\text {aimiR-LUC }}$ or ffgLUC ${ }^{\text {aimiR-PHYB }}$ constructs were subsequently ligated into the Nco I/Not I sites of pIVA2.1 entry vector which contained double 35S promoter and RubescoS terminator. To generate the binary vector, all pIVA2.1-based vectors were cloned into the pKGW_RedSeed vector (Ali et al., 2012) through gateway based site-specific recombination technology with one way LR reaction. The pKGW RedSeed vector contains a DsRed marker gene that is expressed in the seed coat which allows for selection of T0 transformed seeds.

For confirmation of LUC or PHYB silencing in trans the artificial microRNAs 2x35S::amiR-LUC and 2x35S::amiR-PHYB constructs were generated using primer sets which replace the TCP target sequence in miR-319a with target sequences for LUC or PHYB respectively (LUC: CCATGGTATAACTGCCTGCCTCAGATAAGGTCGTGATATGATTCA-F and GCGGCCGCTAGAACTGCCTGCGTCAGATTAAAGAGAATCAATGATCCA-R or PHYB: CCATGGTAGCTGTAAACCGTAAGGCTCAGGTCGTGATATGATTCA-F and GCGGCCGCTAACTGTAAACCGAAAGgCTGAAAGAGAATCAATGATCCA-R). The PCR products were cloned into pIVA2.1 entry vector which was subsequently used for recombination into the pKGW_RedSeed vector. All destination vectors were subsequently transformed to Agrobacterium tumefaciens (AGLO).

\section{Plant transformation and selection transformants}


Agrobacterium tumefaciens was used for plant transformation using the floral dip method as described (Zhang et al., 2006). Transgenic To seeds were identified by DsRed pigmentation of the seed coat. For germination seeds were plated on 3\% water agar plates and cold-treated for 5 days at $4^{\circ} \mathrm{C}$ after which plates were incubated in growth chambers in the light at room temperature. After three days, germinated seedlings were transferred to soil or rock wool for plant growth.

\section{Agrobacterium-mediated transient expression in N.benthamiana leaves}

Agro-infiltration in $N$. benthamiana using agrobacterium strains carrying the different expression vectors (or empty vector) was done as described by Wang (Wang et al., 2016).

\section{LUC activity measurement}

For LUC activity measurements in stable transformed Arabidopsis thaliana plants were sprayed with $1 \mathrm{mM}$ D-luciferin (Duchefa, Haarlem, NL) 24 hour and one hour before imaging with an $\left(-80^{\circ} \mathrm{C}\right)$ air-cooled CCD Pixis 1024B camera system (Princeton Instruments, Massachusetts, USA) equipped with a 35mm, 1:1.4 Nikkon SLR camera lens (Nikon, Tokyo, Japan) fitted with a DT Green filter ring (Image Optics Components Ltd, Orsay, France) to block chlorophyll fluorescence. Exposure time is as indicated.

For transient assays, N.benthamiana leaves were harvested 4 days post agro-infiltration. Leaves were sprayed with $1 \mathrm{mM}$ D-luciferin at $24 \mathrm{hr}$ and $1 \mathrm{hr}$ before imaging (5 minutes exposure time). Relative luminescence from LUC activity was analysed in Image J (Bethesda, Maryland, USA), using background subtraction. For each treatment LUC activity in leaves from 6-8 independent plants was quantified.

Hypocotyl length measurement. For hypocotyl length measurement, seeds were surface sterilized and imbibed on $0.25 \%$ water agar plates at $4^{\circ} \mathrm{C}$ in the dark, after which plates were transferred to a Red LED light box $(50 \mathrm{uMol})$ at $22^{\circ} \mathrm{C}$. Seedlings were flattened at 5 days after transfer, and hypocotyl length was determined from photograph in Image J (Bethesda, Maryland, USA). At least 20 seedlings were scored from each genotype. 
Quantitative RT-PCR. For RNA analysis, $T_{3}$ generation plants were grown for four weeks. The RNA was extracted from rosette leaves from WT (Col-0), ffgLUC del ${ }^{\text {aimiR-319a, }}$ ffgLUC $^{\text {aimiR-319a }}$ or ffgLUC ${ }^{\text {aimiR-PHYB }}$ transformants using InviTrap Spin Plant RNA mini Kit (Berlin, Germany), following manufacturer's instructions. Purified total RNAs were subjected to TURBO DNAfree $^{\mathrm{TM}}$ DNase (Thermo Fisher Scientific Inc., Waltham, Massachusetts) treatment to avoid with contaminated genomic DNA. For reverse transcription the iScrip II mix reagent was used that included 10mM oligo (dT) primer according to the manufacturer's instruction (Bio-Rad, CA,USA). The primers listed in Table S2 were used for the real time qPCR. Reaction were carried out with RNA isolated from pooled samples from three individual plants, with triple biological replicates using SYBR Green PCR Master Mix (Bio-Rad, CA,USA) on the CFX Connect Real Time System machine (Bio-Rad, CA, USA). For Arabidopsis the A. thaliana ACTIN1 was used as reference. RNA analysis from transient assays in $N$. benthamiana were carried out on RNA isolated from three pooled agro-infiltrated leaves, in triple biological replicates, using $N$.benthamiana $U B I 3$ as reference genes. The $C t$ method $(2-\Delta \Delta C t)$ was used to analysis the differences in mRNA values (http://www.bio-rad.com/). All expression constructs used in the transient assays contain a 35S::DsRed marker gene and quantification of the DsRED gene expression in the transient assays was used to confirm similar transformation frequencies in the different agro-infiltration treatments.

\section{Small RNA extraction and stem-loop RT-PCR assays}

Detection of specific small RNAs was by the step-loop PCR method as described by VarkonyiGasic (Varkonyi-Gasic et al., 2007). Briefly, leaf material was collected from ffgLUC (as control), $\mathrm{ffgLUC}_{\text {del }}{ }^{\text {aimiR-319a }}$, ffgLUC aimiR-319a , and ffgLUC ${ }^{\text {aimiR-PHYB }}$ plants and immediately ground in liquid nitrogen with a mortar and pestle. Approximately $100 \mathrm{mg}$ ground leaf tissue was used to small RNA extraction. The extraction of small RNAs were performed by using Prima microRNA Isolation Kit (Lot\#SLBL6958V, Sigma Aldrich, USA) according to the manufacturer's protocol. The small RNA purity and concentration was measured by NanoDrop spectrophotometer (Thermo Scientific, USA).

The specific RT primers were used for miR319a and amiR-PHYB in stem-loop RT reaction. Reverse transcription reaction were performed according to Varkonyi-Gasic et al (Varkonyi- 
Gasic et al., 2007). Forward primers for mature miR319a or amiR-PHYB and universal reverse primer (see Table S2) were used in RT-PCR. The PCR amplification products analysed by gelelectrophoresis on a $4 \%$ agarose gel in 1 XTAE buffer.

Statistical analyses. Comparison of means was analysed for statistical significance with a 2sample t-test $(\mathrm{P}<0.001)$.

\section{Results}

\section{ffgLUC gene with intron-deletion miR-319a (ffgLUC del $^{\text {aimiR-319a }) ~ s h o w s ~ o n l y ~ s i n g l e ~}$ functionality: impaired LUC mRNA splicing but efficient silencing of TCP targets.}

To determine whether a functional microRNA can be efficiently generated from an intron of a transgene, without affecting accuracy of intron splicing, both accuracy and efficiency of transgene splicing and efficiency of silencing by the aimiRNA need to be monitored. To monitor transgene splicing the firefly luciferase (ffgLUC) reporter gene with an intron was used (Luke Mankin et al., 1997) (see Fig. 1 ). To study the efficiency of target gene silencing, the precursor of the native miRNA319a, which targets several members of the Arabidopsis TCP transcription factor family, was used (Palatnik et al., 2007). When the artificial intron-miRNA, aimiR-319a, is correctly processed, it should be active and elicit a leaf growth phenotype similar to that induced by $2 \times 35$ S::miR-319a (Liang et al., 2012). 

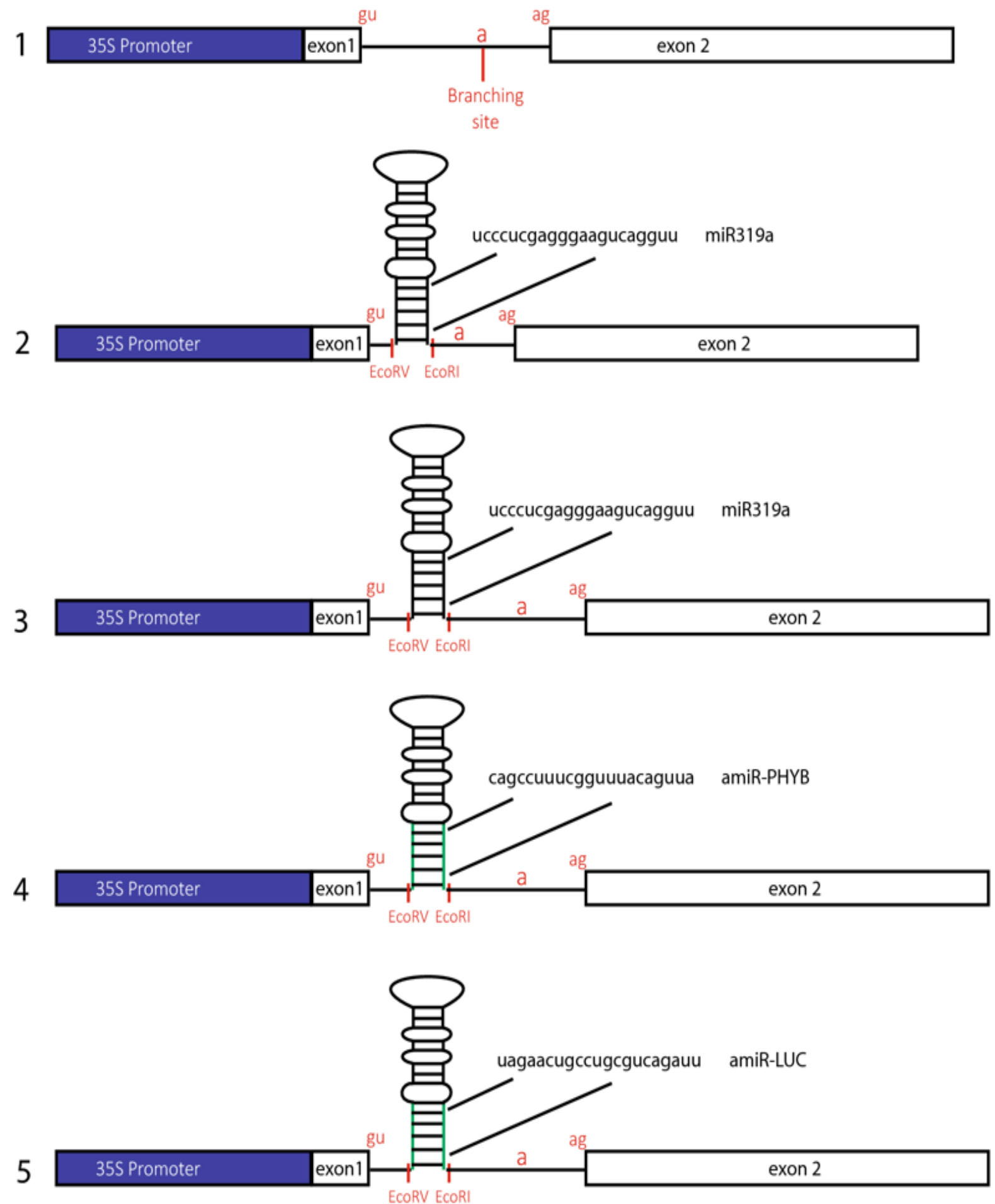

Figure 1. Structure of aimiRNA expression constructs. 1: The Firefly Luciferase gene with intron 2x35S:ffgLUC. 2: aimiRNA gene with miR319a in ffgLUC intron with small deletion: $2 \times 35 \mathrm{~S}: \mathrm{ffgLUC}_{\text {del }}{ }^{\text {aimiR-319a }}$. 3: aimiRNA gene with miR319a in ffgLUC intron without deletion: 2x35S:ffgLUC ${ }^{\text {aimiR-319a }}$. 4: aimiRNA gene targeting

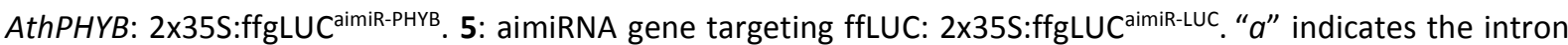
branch point site, " $g u$ " indicates the 5'-splice site and " $a g$ " indicates the 3'-splice site. Exchange of 21 bp target sequence in miR319a for LUC or PHYB target sequences is indicated with green lines. 
The initial cloning procedure for insertion of the miR-319a precursor sequence into the intron of ffgLUC resulted in a 37 base pair deletion in the ffgLUC intron (for sequence see Fig. S1). This gene is named ffgLUC del $^{\text {aimiR-319a }}$ (see Fig. 1-2). In ffgLUC del ${ }^{\text {aimiR-319a }}$ the intron branch point and both 5' and 3' intron border sequences remained intact (see Fig. S1). The ffgLUC del ${ }^{\text {amiR-319a }}$ was cloned into a binary expression vector under control of the enhanced CaMV $2 \times 35 \mathrm{~S}$ promoter and a red seed coat transformation marker gene (Ali et al., 2012). The ffgLUC del $^{\text {aimiR- }}$ ${ }^{319 a}$ expression construct was introduced into Agrobacterium tumefaciens and activity of the constructs was tested both by transient expression in N.benthamiana leaves and by stable transformation of A.thaliana. In the transient expression assay in N. benthamiana, LUC activity of ffgLUC del ${ }_{\text {dimiR-319a }}$ was compared to that of a ffgLUC at 4 days post-agro infiltration. Results show a high LUC activity in leaves expressing ffgLUC, but only low LUC activity for leaves expressing ffgLUC del ${ }^{\text {aimiR-319a }}$ (Fig. 2A). This indicates that intron splicing accuracy from ffgLUC $_{\text {del }}$ aimiR-319a is impaired compared to that of ffgLUC. The transient expression assays in $N$. benthamiana are not suitable to assess if aimiR-319a elicits a leaf phenotype. Therefore, we tested whether endogenous $N$. benthamiana TCP4 (NbTCP4) gene expression was affected by ffgLUC $_{\text {del }}{ }^{\text {aimiR-319a }}$ as the AthTCP target sequence of aimiR-319a shows substantial overlap with sequences in NbTCP4. Results show that NbTCP4 expression level was reduced in leaves expressing ffgLUC del $^{\text {aimiR-319a }}$ compared to the control leaves expressing ffgLUC (Fig. 2A).

Transformants of Arabidopsis thaliana with the ffgLUC del $^{\text {aimiR-319a }}$ or ffgLUC expression constructs were identified in $\mathrm{T}_{0}$ seeds by expression of the red seed coat marker present in the binary vector (Table S1). From the red ffgLUC del ${ }^{\text {aimiR-319a }} \mathrm{T}_{0}$ seeds 19 independent transformants were grown. All these plants showed a leaf growth phenotype (data not showed) as described for plants expressing 2x35S::miR319a (Liang et al., 2012), indicating that the miR319a is efficiently processed from aimiR-319a in stable transformed plants. Indeed, expression analysis of the miR319a target genes AthTCP2, AthTCP3, AthTCP4 indicated that their expression was reduced by $\sim 90 \%$ in the ffgLUC del $^{\text {aimiR-319a }}$ plants (Fig. 2B). 


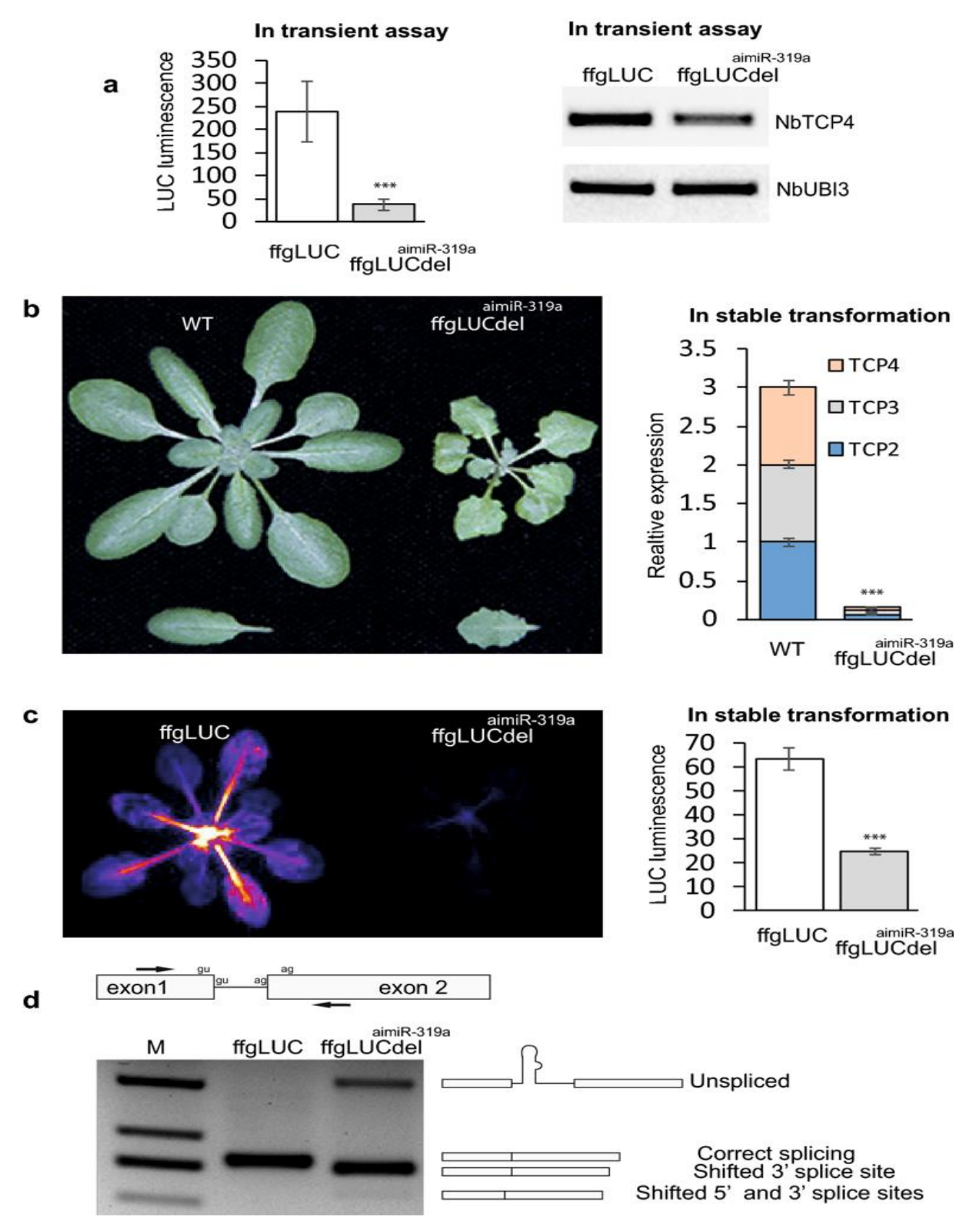

Figure 2. Single activity of ffgLUC $\mathrm{Cel}^{\text {aimiR-319a }}$ in transient assays and stable transformed plants.

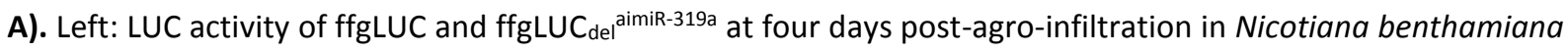
transient assay. Significant differences between samples $\left({ }^{* * *}\right)$ is based on standard error (student's $t$-test, $P<0.01$ ). Right: RT-PCR analysis of NbTCP4 expression in transient assay with ffgLUC or ffgLUCdel ${ }^{\text {aimiR-319a. }}$. Quantification of the DSRED gene expression was used to confirm similar transformation efficiencies in the agroinfiltration with ffgLUC and ffgLUCdel ${ }^{\text {aimiR-319a }}$ (see Fig. S3).

B). Left: Representative stable transformed Arabidopsis rosette plant and leaf expressing ff-gLUC or ffgLUC del ${ }^{\text {aimiR- }}$ 319a. Right: (reference gene AthActin1, expression of TCP2,3 and 4 each normalized to that in one WT plant). Significant differences between samples $\left({ }^{* *}\right)$ is based on standard error (student's $t$-test, $\mathrm{P}<0.01$ ). 
C). LUC activity in representative stable transformant Arabidopsis expressing ff-gLUC or ffgLUC del ${ }^{\text {aimiR-319a }}$. Graph: quantified LUC expression of eight independent transformants expressing either ff-gLUC or ffgLUC del ${ }^{\text {aimiR-319a }}$.

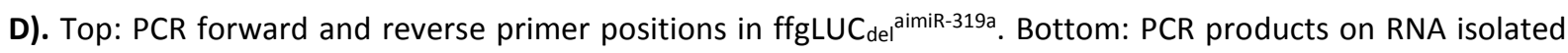
from ffgLUC del ${ }^{\text {aimiR-319a }}$ plants. At each band position the structure of the mRNA sequence is shown (see Fig. S2).

However, the LUC activity in plants expressing ffgLUC del $^{\text {aimiR-319a }}$ is low compared to control plants (expressing ffgLUC) of the same age (Fig. 2C). Both the reduced LUC activity of ffgLUC $_{\text {del }}{ }^{\text {aimiR-319a }}$ in transient assay and stable transformants suggest an incorrect maturation

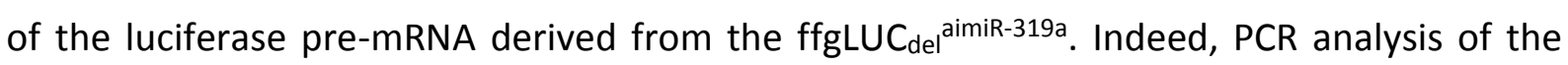
luciferase mRNA across the intron splice site showed that there were multiple aberrant products and only very low levels of correctly spiced luciferase mRNA derived from ffgLUC del $^{\text {aimiR-319a }}$ (Fig. 2D). Presumably, the dual action at the intron in luciferase pre-mRNA by both an intron-splicing protein-complex and an miRNA processing protein-complex leads to spatial interference, which in this case especially affects correct maturation of the pre-mRNA. Sequence analysis of the aberrant PCR products showed that both aberrant 3'- and 5' splice site selection occurred, while the major PCR product was derived from unspliced mRNA (Fig. $2 \mathrm{D}$ and Fig. S2). To solve the putative spatial interference during processing of $\mathrm{ffg}_{\mathrm{LU}} \mathrm{C}_{\mathrm{del}}{ }^{\text {aimiR- }}$ 319a mRNA maturation, we next adapted the cloning strategy for miRNA insertion into the intron.

\section{ffgLUC ${ }^{a i m i R-319 a}$ displays dual functionality: correct LUC mRNA splicing and TCP silencing.}

The miRNA insertion cloning strategy was adapted by direct insertion of the aimiRNA into the ffgLUC intron, without deletion of intron sequence, resulting in the expression construct

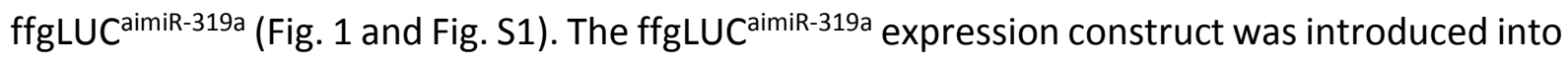
Agrobacterium tumefaciens and was again tested both by transient expression in $N$. benthamiana and by stable transformation of Arabidopsis. In the transient expression assays, the activity of ffgLUC aimiR-319a was compared with that of ffgLUC without intronic miR319a (Fig. $3 A)$. This resulted in a similar LUC activity in leaf tissue expressing either ffgLUC aimiR-319a or ffgLUC, suggesting an efficient and accurate splicing of the intron from ffgLUCaimiR-319a mRNA (Fig. 3A). To test the functionality of aimiR-319a in targeting TCP genes in N. benthamiana, 
NbTCP4 mRNA level was checked by RT-PCR in control treatments and leaves expressing ffgLUCmaimiR-319a. NbTCP4 expression was reduced by $60 \%$ in leaves infiltrated with ffgLUC CimiRNA319a, suggesting that a functional miRNA319a can be produced from aimiR-319a (Fig. 3A).

a

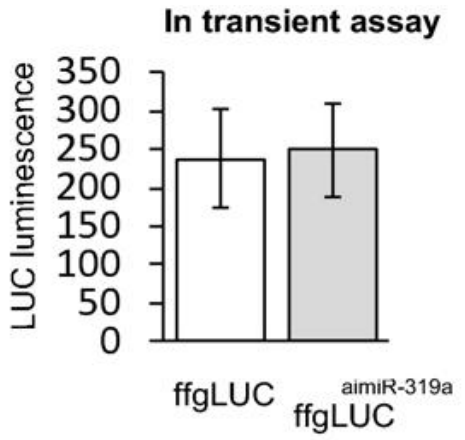

In transient assay

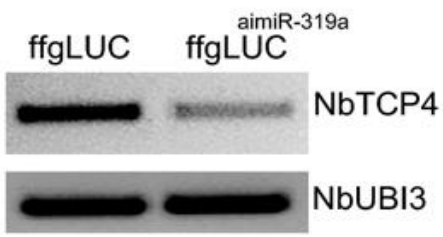

b

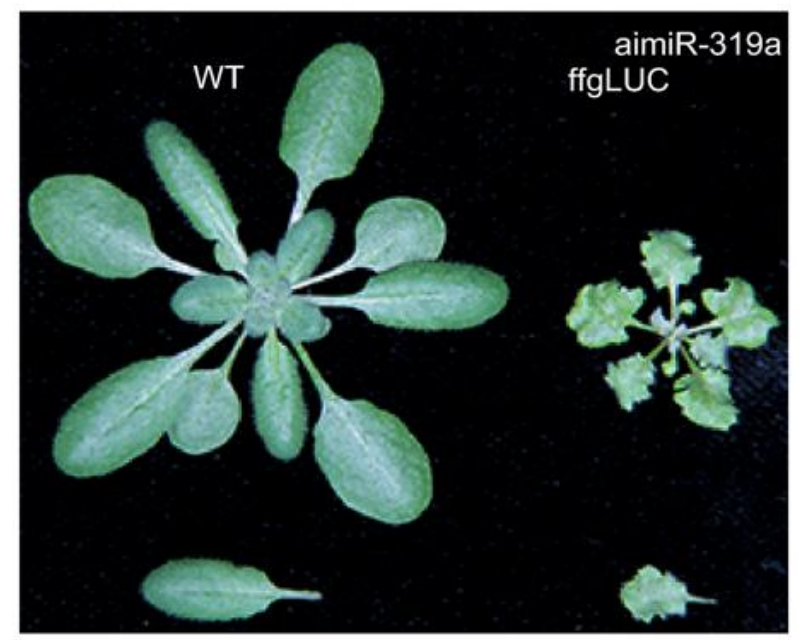

C

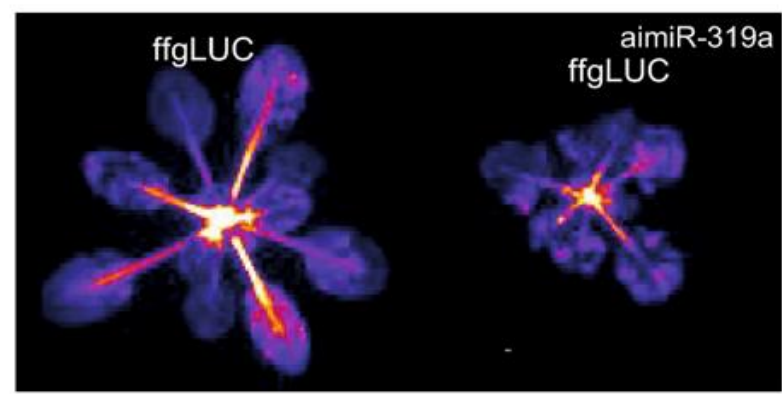

In stable transformation

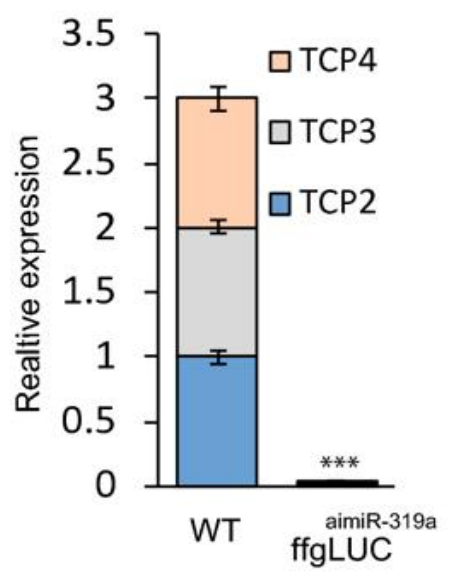

In stable transformation

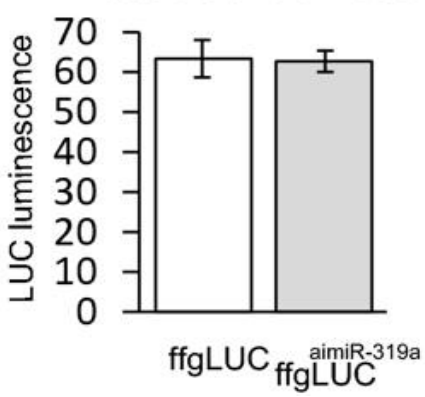

Figure 3. Dual activity of ffgLUC ${ }^{\text {aimiR-319a }}$ in transient assays (A) and stable transformed plants (B and C).

A). Left: LUC activity of ffgLUC and ffgLUC ${ }^{\text {aimiR-319a }}$ at four days post-agro-infiltration in Nicotiana benthamiana transient assay ( $\mathrm{n}=$ five leaves per treatment). Right: RT-PCR analysis of NbTCP4 expression in transient assay with

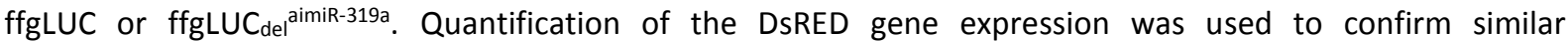
transformation efficiencies in the agro-infiltration with ffgLUC and ffgLUC ${ }^{\text {aimiR-319a }}$ (see Fig. S3). 
B). Left: Representative stable transformed Arabidopsis thaliana rosette plant and leaf expressing ff-gLUC or ffgLUC $C^{\text {aimiR-319a }}$ Right: average relative expression level of $T C P 2 / 3 / 4$ genes in five WT and ffgLUC del ${ }^{\text {aimiR-319a }}$ plants (reference gene AthActin1, expression of TCP2,3 and 4 each normalized to that in one WT plant). Significant differences between samples $\left({ }^{* * *}\right)$ is based on standard error (student's $t$-test, $\mathrm{P}<0.01$ ).

C). Left: LUC activity in representative stable transformant Arabidopsis thaliana expressing ff-gLUC or ffgLUC ${ }^{\text {aimiR- }}$ 319a. Right: quantified LUC in expression of eight independent transformants expressing either ff-gLUC or ffgLUC aimiR-319a.

From the stable transformation of Arabidopsis, $\mathrm{T}_{0}$ seeds expressing the red seed coat marker were selected, from which 19 independent T1 transformants were grown (Table S1). Out of these 19 plants, two plants did not survive, while 17 plants produced $T_{1}$ seeds. Each of these $17 T_{1}$ plants showed the phenotype associated with constitutively overexpressed native miR319a (Liang et al., 2012; Alvarez et al., 2016) (Fig. 3B). This indicates that an miR319a was efficiently processed from imiR-319a located in the intron of ffgLUCaimiR-319a in stably transformed plants, leading to efficient silencing of TCP genes. This is also confirmed by qPCR analysis of RNA isolated from a representative ffgLUC aimiR-319a transformant, which shows $>90 \%$ reduction in TCP2, TCP3 and TCP4 mRNA levels compared to plants expressing conventional ffgLUC (Fig. 3B). Nevertheless, LUC activity in the same ffgLUC aimiR-319a transformant is similar compared to the ffgLUC control (Fig. 3C). These results indicate that the luciferase pre-mRNA is correctly spliced and simultaneously aimiR-319a provides silencing of TCPs in transformed plants.

\section{aimiR-LUC silences ffgLUC in-cis in stable transformants, but not in transient assays.}

An aimiRNA was made targeting the luciferase mRNA itself (aimiR-LUC). The aimiR-LUC is based on the sequence and structure of the native miR319a precursor, but the 21 base-pair sequences targeting TCPs are replaced by 21 base-pairs targeting luciferase mRNA (Fig. 1 and Fig. S1). In cells expressing ffgLUC aimiR-LUC, aimiR-LUC targets expression of the LUC transgene from which it is derived (silencing in-cis). Both mature aimiR-LUC and LUC mRNA are produced from the same pre-mRNA and silencing of LUC activity provides information on the relative efficiency of the two maturation processes (mRNA vs miRNA). In the transient expression 
assays, LUC activity in $N$. benthamiana leaves expressing ffgLUC aimiR-LUC showed no significant reduction compared with leaves expressing ffgLUC (Fig. 4A). This indicates correct splicing of the luciferase pre-mRNA, but no effective silencing by aimiR-LUC in-cis. To compare the silencing in-cis with silencing in-trans in the transient assay, an ffCLUC (LUC CDNA) expression construct was co-infiltrated with a 2x35S::amiR-LUC expression construct. This showed that also 2x35S::amiR-LUC is not capable of silencing transiently expressed LUC (Fig. 4A). Combined, these results indicate that efficient maturation of luciferase mRNA from ffgLUC CimiR-LUC occurs upon transient expression but that silencing by aimiR-LUC or amiR-LUC is not effective under these conditions.

The ffgLUC and ffgLUCaimiR-LUC binary vectors were also stably transformed into Arabidopsis and $T_{0}$ seeds with the red seed coat were identified (Table S1). For each transformation event, 16 independent transformants were grown and LUC activity was quantified in independent transformed plants at 21 days post germination. On average, the LUC activity was reduced by $65 \%$ in the 16 individual ffgLUCaimiR-LUC $T_{1}$ plants compared to that in 16 individual $T_{1} \mathrm{ffgLUC}$ plants (Fig. 4B). This indicates that amiR-LUC is efficiently processed from aimiR-LUC in stably transformed plants. For comparison of silencing in-cis with silencing in-trans in stably transformed plants, one line expressing 2x35S::ffcLUC was transformed with a 2x35S::amiRLUC expression construct. In three T2 double transformants (homozygous for both 2x35S::ffcLUC and 2x35S::amiR-LUC) the LUC activity was reduced by $69-53 \%$ compared to the original ffcLUC line (Fig. 4C). Silencing efficiency in-trans therefore seems to be in the same range as silencing efficiency in-cis. 
a In transient assay

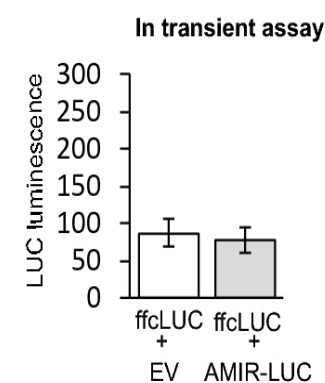

b 70

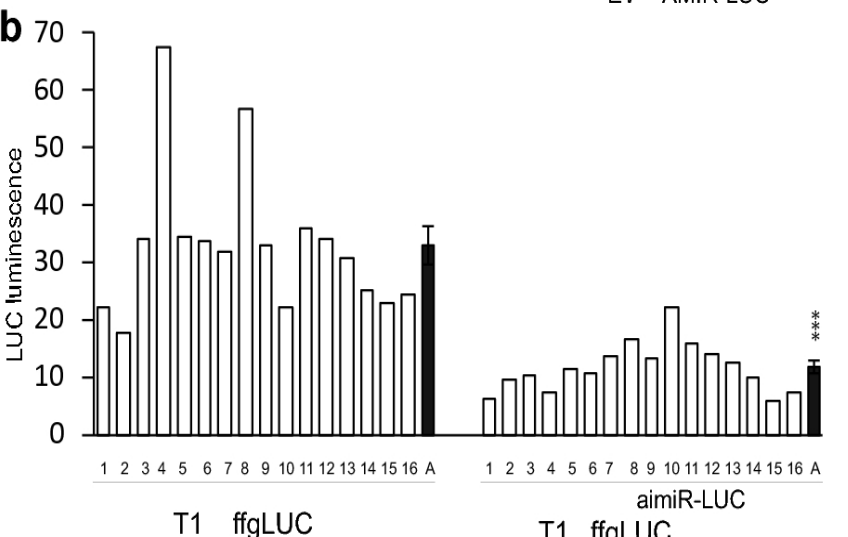

T1 ffgLUC

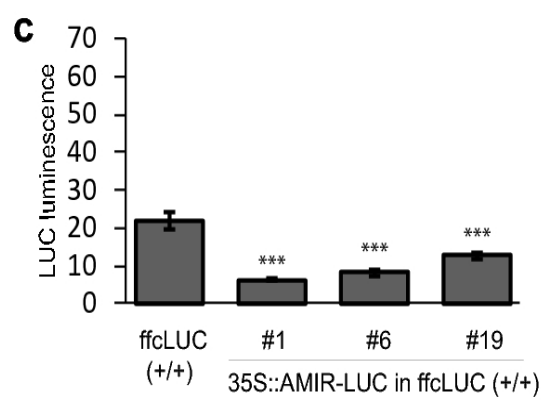

Figure 4. Evaluation of silencing in cis- and trans in transient assays (A) and stable transformed plants (B and C).

A). Left: evaluation silencing in-cis transient assay: LUC activity of ffgLUC and ffgLUC aimir-LUC at four days post-agro-infiltration in Nicotiana benthamiana transient assay ( $\mathrm{n}=$ five leaves per treatment). Right: evaluation silencing in-trans transient assay: LUC activity in Nicotiana benthamiana transient assay of ffcLUC coinfiltrated with empty vector and ffCLUC coinfiltrated with amiR-LUC at four days post-agroinfiltration ( $\mathrm{n}=$ five leaves per treatment). Quantification of the DsRED gene expression was used to confirm similar transformation efficiencies in the agro-infiltration with ffgLUC and ffgLUC ${ }^{\text {aimiR- }}$ Luc (see Fig. S3). B). Evaluation of silencing in-cis: Relative LUC activity in sixteen independent $T_{1}$ generation of ffgLUC and ffgLUC Camir-LUC plants. " $A$ " indicates average LUC activity in set of transgenic plants. Significant differences between samples

$\left({ }^{* * *}\right)$ is based on standard error (student's $t$-test, $\left.\mathrm{P}<0.01\right)$. C). Evaluation of silencing in-trans: Relative LUC activity in homozygous ffCLUC line and T1 of same ffcLUC line transformed with $2 \times 35$ S:amiR-LUC. Significant differences between samples $\left({ }^{* *}\right)$ is based on standard error (student's $t$-test, $\mathrm{P}<0.01$ ).

\section{ffgLUC aimiR-PHYB shows efficient mRNA maturation, but no silencing of AthPHYB.}

In addition to the aimiRNA targeting TCP and LUC, an aimiRNA targeted against the PHYB mRNA of Arabidopsis thaliana was tested. The aimiR-PHYB was again placed at the same intron position as in the functional ffgLUC ${ }^{\text {imiR-319a }}$ and ffgLUC aimiR-LUC constructs. The aimiR-LUC is based again on the miR319a but with a replacement of the 21 base pairs in miR319a targeting TCP by 21 base pairs targeting AthPHYB mRNA (ffgLUC ${ }^{\text {aimiR-PHYB }) ~(F i g . ~} 1$ and Fig. S1). In transient assays, the leaf tissues expressing ffgLUC ${ }^{\text {aimiR-PHYB }}$ showed similar LUC activity as leaves expressing ffgLUC (Fig. 5A), again indicating efficient and accurate maturation of the luciferase pre-mRNA from the ffgLUC ${ }^{a i m i R-P H Y B}$ expression construct. 

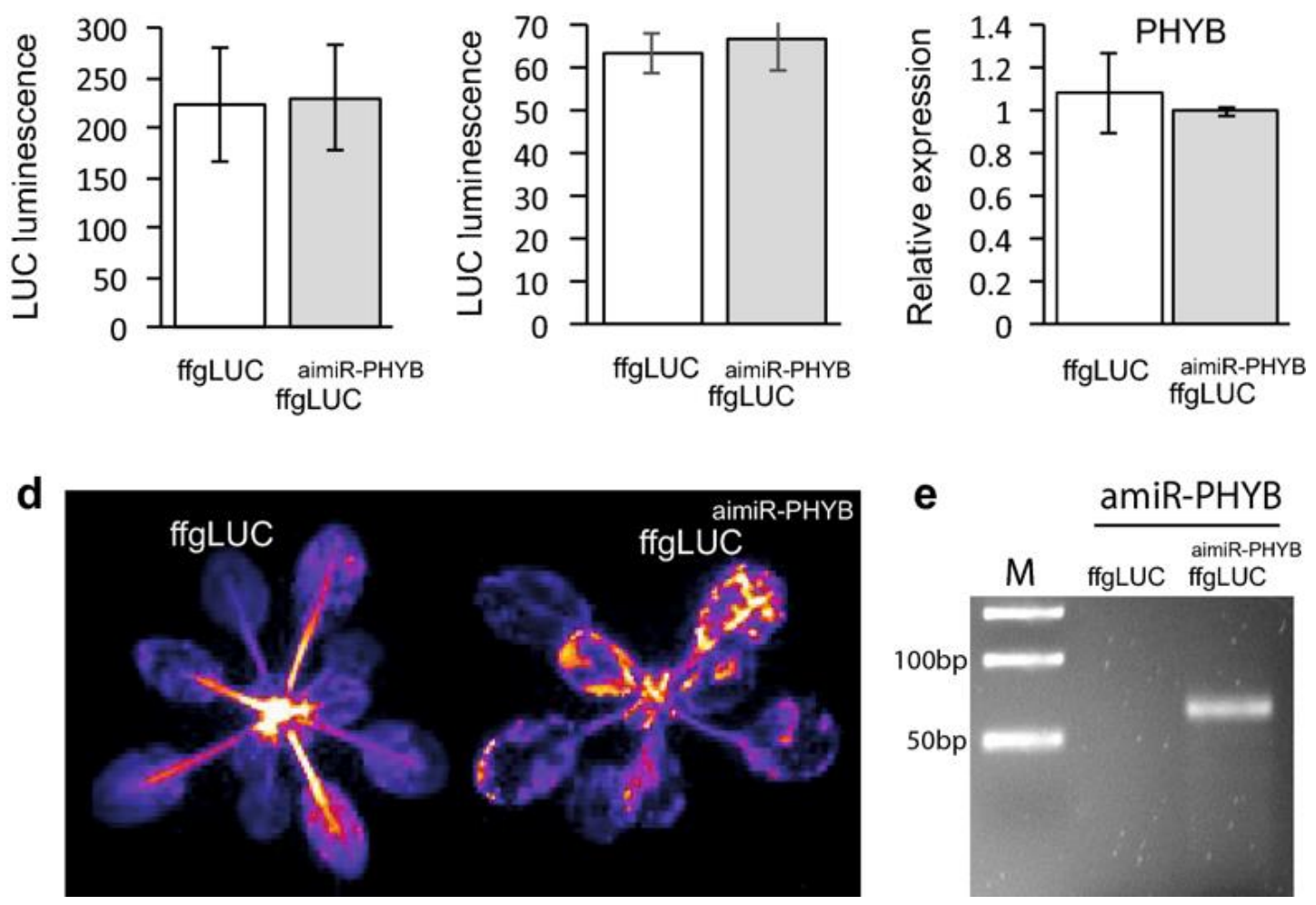

Figure 5. Activity of ffgLUC ${ }^{\text {aimiR-PHYB }}$ in transient assays (A) and stable transformed plants (B and C).

A). Relative LUC activity of ffgLUC and ffgLUC ${ }^{\text {aimiR-PHYB }}$ at four days post-agro-infiltration of Nicotiana benthamiana leaves ( $\mathrm{n}=$ five leaves per treatment);

B). Relative LUC activity in eight independentstable transformants of Arabidopsis thaliana expressing either ffgLUC or ffgLUC CaimiR-PHYB;

C). Average relative expression level of AthPHYB in five ff-gLUC and ffgLUC ${ }^{\text {aimir-PHYB }}$ plants (reference gene AthActin1, expression of AthPHYB normalized to that in one ffgLUC plant);

D). Image of LUC activity in representative transgenic plants expressing ff-gLUC (left) or ffgLUC ${ }^{\text {aimir-PHYB }}$ (right);

E). Detection of mature amiR-PHYB by stem-loop RT PCR analysis in small RNA isolated from ffgLUC ${ }^{\text {aimiR-PHYB }}$ plants but not in small RNA isolated from control ffgLUC plants.

After stable transformation of Arabidopsis thaliana with ffgLUC aimiR-PHYB, $\mathrm{T}_{0}$ seeds with the red seed coat were identified (Table 1 ) and 10 independent transformed $\mathrm{T}_{1}$ plants were grown. From these, eight transformants with a single copy transgene insertion were selected for further analysis. On average, the LUC activity in these eight lines was comparable with that of eight independent transformants expressing ffgLUC (Fig. 5B). This again indicates that also the 
intron in ffgLUC ${ }^{\text {aimiR-PHYB }}$ is efficiently and correctly spliced from the luciferase pre-mRNA. In contrast, the silencing of PHYB is not effective in ffgLUC aimiR-PHYB lines as expression of PHYB mRNA is similar in ffgLUC aimiR-PHYB and ffgLUC lines (Fig. 5C). The silencing of PHYB mRNA expression can also be tested in a bioassay. When seedlings are grown under constant Red (CR) light, lines with reduced PHYB expression are expected so show enhanced hypocotyl elongation. Ten independent homozygous T1 lines of ffgLUC ${ }^{\text {aimiR-PHYB }}$ were germinated under cR. While the hypocotyl length of a phyB-9 mutant was elongated compared with WT, the hypocotyl length of the ten ffgLUC aimiR-PHYB transformants was not statistically different from WT (data not shown). All together, these results suggest that the mature amiR-PHYB derived from ffgLUC ${ }^{a i m i R-P H Y B}$ is not functional in silencing PHYB expression.

\section{Discussion}

\section{Functional aimiRNA requires sufficient spacing in intron.}

Intron-derived miRNAs (imiRNAs) are an alternative source for miRNAs in mammals and plants (Berezikov et al., 2007; Ruby et al., 2007; Meng and Shao, 2012; Sibley et al., 2012; Tong et al., 2013). Evidence has been obtained that functional miRNAs can be derived from imiRNAs in mammalian cells and plants (Naqvi et al., 2012; Ha and Kim, 2014) but plant genes containing intronic miRNA sequences have only been studied sparsely. Here, we demonstrate that the concept of an imiRNA can be used to construct a transgene with dual functionality: overexpression of the transgene and silencing of an endogenous target gene of interest. Our constructs demonstrate that the structural sequence information of the pre-miRNA mi319a is sufficient for full functionality when placed correctly into an intron, allowing for both normal maturation of the pre-mRNA and for generation of a functional mature microRNA.

In all aimiRNA constructs tested here, the aimiRNA was inserted into an 189 long intron sequence of the ffgLUC gene. In the first construct the insertion was done at $10 \mathrm{bp}$ from the 5 '-end of the LUC-intron sequence. For this construct the LUC activity was low compared to ffgLUC control construct in both in transient and stable (Fig. 2A,C). This indicates that a certain 
distance is needed between the $5^{\prime}$-splice site and the imiRNA insertion site for efficient premRNA maturation. It could be that the reduced distance between $5^{\prime}$-splice site and imiRNA sequence in $\mathrm{ffgLUC}_{\text {del }}$ aimiR-319a resulted in spatial constraints because of simultaneous assembly of spliceosome and miRNA-processing protein complexes. In contrast, the aimiRNA placed at $55 \mathrm{bp}$ from the $5^{\prime}$-splice site resulted in efficient maturation of the LUC mRNA, resulting in similar LUC activity for ffgLUC aimiRNA-319a and ffgLUC in transient expression as well as stable transformants (Fig. 3A,C). It was not investigated whether mRNA and aimiRNA derive from the same pre-mRNA transcript or whether the two mature products are produced mutually exclusive. However, since LUC activity from ffgLUC aimiRNA-319a is similar as from ffgLUC it suggests the same level of mRNA production from both constructs. If part of the pre-mRNA is exclusively used for mature amiRNA production and the other part for mature ffgLUC mRNA

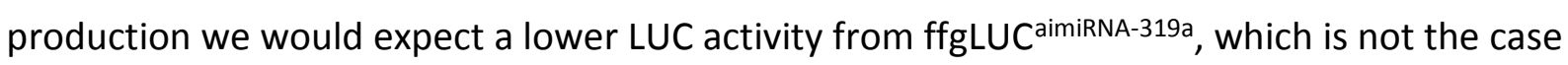
(Fig. 3). Whether both products (mRNA, aimiRNA) are indeed derived from the same premRNA needs further investigation but for practical purposes the ffgLUC aimiRNA constructs seem to function as dual functional transgenes.

The imiRNA positioning within the intron may be further improved for functionality, for which positioning of natural imiRNA in plant genes may be used as a guide. The average length of introns is $101 \mathrm{bp}$ in Arabidopsis and $160 \mathrm{bp}$ in rice (Wang and Brendel, 2006; Schuler, 2008). By contrast, the average length of introns containing imiRNAs is $625 \mathrm{bp}$ in Arabidopsis and $2178 \mathrm{bp}$ in rice (Yang et al., 2012). Therefore, it may still be possible that a larger distance between inserted miRNA and the 5'-and 3' splice sites enhances functionality of the imiRNA (more efficient splicing and processing to miRNA).

\section{Efficiency of silencing is function of both aimiRNA and target gene expression level.}

The construct ffgLUCaimiRNA-LUC with the miRNA targeting the LUC mRNA in-cis showed 65\% reduction in LUC activity, which is very similar to the silencing in-trans reached by a 2x35S::amiR-LUC in stably transformed plants (Fig. 4B,C). Presumably the LUC mRNA and aimiR-LUC are produced in equal molar amounts from ffgLUC aimiRNA-LUC pre-mRNA, suggesting that miRNA needs to be in excess to its target mRNA in order to obtain higher levels of silencing. For instance, silencing of the TCP transcription factor genes, which are expressed at 
low levels, by the aimiRNA is very efficient (Fig. 2B and 3B). In contrast to the stable transformed plants, the constructs targeting LUC mRNA in-cis or in-trans are not effective in transient assays. In transient assays the gene copy number is artificially high and may result in saturation of the gene silencing machinery.

\section{amiRNA-PHYB not functional because of target mRNA secondary structure?}

The construct ffgLUC aimiRNA-PHYB showed correct splicing but this did not result in significant down regulation of PHYB mRNA levels in transformed Arabidopsis. Analysis of small RNA isolated from the plants expressing ffgLUC aimiRNA-PHYB by stem-loop PCR (Varkonyi-Gasic et al., 2007) with specific primers did show that the expected aimiRNA ${ }^{\text {PHYB }}$ product is produced in these plants (Figure 5E), but apparently it is not active against the PHYB mRNA.
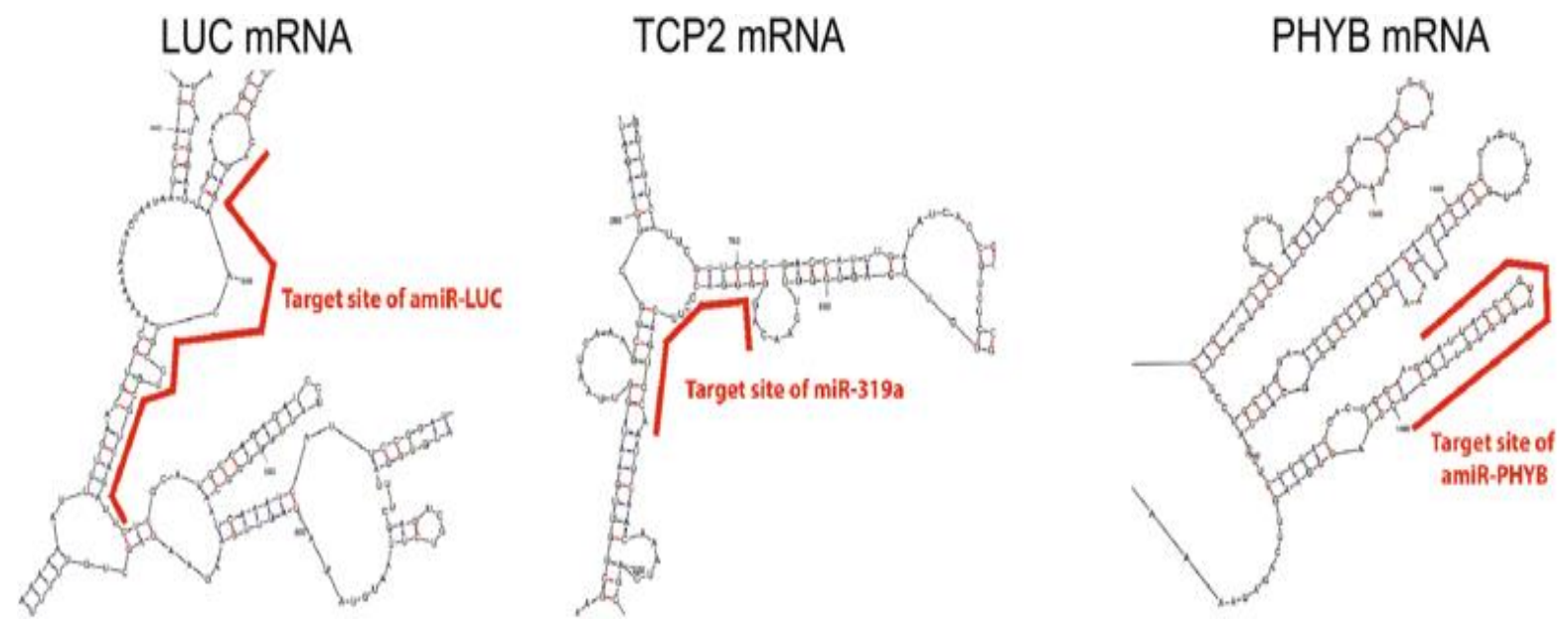

Figure 6. Predicted secondary structure of mRNAs targeted by miRNA. RNA secondary structure prediction by UNAFold (http://unafold.rna.albany.edu/) (Buratti and Baralle, 2004).

Also when the same amiR-PHYB was expressed directly from a 2x35S-promoter, transformants did not show a PHYB silencing phenotype under constant cR (Fig. S5). The lack of silencing by either aimiR-PHYB or amiR-PHYB suggests that the PHYB target sequence cannot be effectively silenced. For selection of the PHYB miRNA target sequence the WMD3-Web MicroRNA Designer online tool was used, which selects the best target sequence based on both target and off-target sequences (Schwab et al., 2006). However, recently it was shown that 
effectiveness of miRNA sequences also depends on the secondary structure of the target mRNA (Zheng et al., 2017). Indeed, when the secondary structure of the target mRNA is taken into account (Buratti and Baralle, 2004), it turns out that both the amiR-LUC and native miR319a target the mRNA at a part that is largely single stranded. In contrast, the chosen amiR-PHYB sequence targets the PHYB mRNA at an internal stem loop structure (Fig. 6). Possibly, this explains why the ffgLUC ${ }^{\text {aimiR-PHYB }}$ construct does not show effective silencing of PHYB.

In conclusion, the method with transgenes containing an amiRNA in their intron allows for combining ectopic overexpression of the transgene with silencing of a target gene of interest. Artificial miRNA genes containing functional clusters of miRNAs have been engineered (Wang et al., 2016). Therefore, our concept of transgenes containing aimiRNA may be extended by multiple aimiRNAs in a single intron or in different introns in the same transgene provided that these aimiRNAs are still efficiently processed and allow dual/multiple functionality of the transgene.

\section{List of abbreviations}

imiRNA: intronic microRNA, aimiRNA: artificial intronic microRNA, ffgLUC: firefly genomic luciferase, TCP: TEOSINTE BRANCHED1/CYCLOIDEA/PROLIFERATING CELL FACTOR1, PHYB: PHYTOCHROME B, 5'ss: 5' splice sites

\section{Declarations:}

\section{Authors' contributions}

US developed the method, carried out the experimental work and drafted the manuscript. MvH made Luminator system and analysed the data. MS developed the plant material. IA and HB helped writing the manuscript. SvdK supervised manuscript writing and the project.

\section{Consent for publication}

All authors read and approved the final manuscript. 


\section{Competing interests}

The authors declare that they have no competing interests.

\section{Ethics approval and consent to participate}

Not applicable.

\section{Funding}

This research is funded by the STW project (13149) 'Compact Plants'.

\section{Acknowledgements}

The first author would like to thank the Erasmus Mundus Action 2 projects TIMUR.

\section{References}

Ali MA, Shah KH, Bohlmann H (2012) pMAA-Red: a new pPZP-derived vector for fast visual screening of transgenic Arabidopsis plants at the seed stage. BMC Biotechnology 12: 37

Alvarez JP, Furumizu C, Efroni I, Eshed Y, Bowman JL (2016) Active suppression of a leaf meristem orchestrates determinate leaf growth. Elife $\mathbf{5}$

Bartel DP (2004) MicroRNAs: genomics, biogenesis, mechanism, and function. Cell 116: 281297

Berezikov E, Chung W-J, Willis J, Cuppen E, Lai EC (2007) Mammalian Mirtron Genes. Molecular Cell 28: 328-336

Buratti E, Baralle FE (2004) Influence of RNA Secondary Structure on the Pre-mRNA Splicing Process. Molecular and Cellular Biology 24: 10505-10514

Chen Y, Han Y, Zhang M, Zhou S, Kong X, Wang W (2016) Overexpression of the Wheat Expansin Gene TaEXPA2 Improved Seed Production and Drought Tolerance in Transgenic Tobacco Plants. PLOS ONE 11: e0153494

Ha M, Kim VN (2014) Regulation of microRNA biogenesis. Nat Rev Mol Cell Biol 15: 509-524

Karaba A, Dixit S, Greco R, Aharoni A, Trijatmiko KR, Marsch-Martinez N, Krishnan A, Nataraja KN, Udayakumar M, Pereira A (2007) Improvement of water use efficiency in rice by expression of HARDY, an Arabidopsis drought and salt tolerance gene. Proc Natl Acad Sci U S A 104: 15270-15275

Kashyap N, Pham B, Xie Z, Bleris L (2013) Transcripts for combined synthetic microRNA and gene delivery. Molecular bioSystems 9: 1919-1925 
Kasuga M, Liu Q, Miura S, Yamaguchi-Shinozaki K, Shinozaki K (1999) Improving plant drought, salt, and freezing tolerance by gene transfer of a single stress-inducible transcription factor. 17: 287-291

Knop K, Stepien A, Barciszewska-Pacak M, Taube M, Bielewicz D, Michalak M, Borst JW, Jarmolowski A, Szweykowska-Kulinska Z (2017) Active 5' splice sites regulate the biogenesis efficiency of Arabidopsis microRNAs derived from intron-containing genes. Nucleic Acids Research 45: 2757-2775

Liang G, He H, Li Y, Yu D (2012) A new strategy for construction of artificial miRNA vectors in Arabidopsis. Planta 235: 1421-1429

Lin SL, Miller JD, Ying SY (2006) Intronic microRNA (miRNA). J Biomed Biotechnol 2006: 26818

Luke Mankin S, Allen GC, Thompson WF (1997) Introduction of a plant intron into the luciferase gene ofPhotinus pyralis. Plant Molecular Biology Reporter 15: 186-196

Meng Y, Shao C (2012) Large-scale identification of mirtrons in Arabidopsis and rice. PLoS One 7: e31163

Nag A, King S, Jack T (2009) miR319a targeting of TCP4 is critical for petal growth and development in Arabidopsis. Proc Natl Acad Sci U S A 106: 22534-22539

Naqvi AR, Sarwat M, Hasan S, Roychodhury N (2012) Biogenesis, functions and fate of plant microRNAs. J Cell Physiol 227: 3163-3168

Palatnik JF, Wollmann H, Schommer C, Schwab R, Boisbouvier J, Rodriguez R, Warthmann N, Allen E, Dezulian T, Huson D, Carrington JC, Weigel D (2007) Sequence and expression differences underlie functional specialization of Arabidopsis microRNAs miR159 and miR319. Dev Cell 13: 115-125

Park W, Zhai J, Lee JY (2009) Highly efficient gene silencing using perfect complementary artificial miRNA targeting AP1 or heteromeric artificial miRNA targeting AP1 and CAL genes. Plant Cell Rep 28: 469-480

Parsi S, Soltani BM, Hosseini E, Tousi SE, Mowla SJ (2012) Experimental Verification of a Predicted Intronic MicroRNA in Human NGFR Gene with a Potential Pro-Apoptotic Function. PLOS ONE 7: e35561

Peng J, Richards DE, Hartley NM, Murphy GP, Devos KM, Flintham JE, Beales J, Fish U, Worland AJ, Pelica F, Sudhakar D, Christou P, Snape JW, Gale MD, Harberd NP (1999) 'Green revolution' genes encode mutant gibberellin response modulators. Nature 400: 256-261

Prelich G (2012) Gene Overexpression: Uses, Mechanisms, and Interpretation. Genetics 190: 841-854

Reinhart BJ, Weinstein EG, Rhoades MW, Bartel B, Bartel DP (2002) MicroRNAs in plants. Genes Dev 16: 1616-1626

Ren G, Yu B (2012) Post-transcriptional control of miRNA abundance in Arabidopsis. Plant Signal Behav 7: 1443-1446

Ruby JG, Jan CH, Bartel DP (2007) Intronic microRNA precursors that bypass Drosha processing. 448: 83-86

Schuler MA (2008) Splice Site Requirements and Switches in Plants. In Nuclear pre-mRNA Processing in Plants. Springer Berlin Heidelberg, Berlin, Heidelberg, pp 39-59

Schwab R, Ossowski S, Riester M, Warthmann N, Weigel D (2006) Highly specific gene silencing by artificial microRNAs in Arabidopsis. Plant Cell 18: 1121-1133 
Sibley CR, Seow Y, Saayman S, Dijkstra KK, El Andaloussi S, Weinberg MS, Wood MJA (2012) The biogenesis and characterization of mammalian microRNAs of mirtron origin. Nucleic Acids Research 40: 438-448

Tong YA, Peng H, Zhan C, Fan L, Ai T, Wang S (2013) Genome-wide analysis reveals diversity of rice intronic miRNAs in sequence structure, biogenesis and function. PLoS One 8: e63938

Varkonyi-Gasic E, Wu R, Wood M, Walton EF, Hellens RP (2007) Protocol: a highly sensitive RT-PCR method for detection and quantification of microRNAs. Plant Methods 3: 1212

Wang B, Kashkooli AB, Sallets A, Ting H-M, de Ruijter NCA, Olofsson L, Brodelius P, Pottier M, Boutry M, Bouwmeester H, van der Krol AR (2016) Transient production of artemisinin in Nicotiana benthamiana is boosted by a specific lipid transfer protein from A. annua. Metabolic Engineering 38: 159-169

Wang BB, Brendel V (2006) Genomewide comparative analysis of alternative splicing in plants. Proc Natl Acad Sci U S A 103: 7175-7180

Wang T, Xie Y, Tan A, Li S, Xie Z (2016) Construction and Characterization of a Synthetic MicroRNA Cluster for Multiplex RNA Interference in Mammalian Cells. ACS Synth Biol 5: $1193-1200$

Watson E, MacNeil Lesley T, Arda HE, Zhu Lihua J, Walhout Albertha JM (2013) Integration of Metabolic and Gene Regulatory Networks Modulates the C. elegans Dietary Response. Cell 153: 253-266

Yan K, Liu P, Wu CA, Yang GD, Xu R, Guo QH, Huang JG, Zheng CC (2012) Stress-induced alternative splicing provides a mechanism for the regulation of microRNA processing in Arabidopsis thaliana. Mol Cell 48: 521-531

Yang GD, Yan K, Wu BJ, Wang YH, Gao YX, Zheng CC (2012) Genomewide analysis of intronic microRNAs in rice and Arabidopsis. J Genet 91: 313-324

Zhang X, Henriques R, Lin S-S, Niu Q-W, Chua N-H (2006) Agrobacterium-mediated transformation of Arabidopsis thaliana using the floral dip method. 1: 641-646

Zheng Z, Reichel M, Deveson I, Wong G, Li J, Millar AA (2017) Target RNA secondary structure is a major determinant of miR159 efficacy. Plant Physiology 


\section{Supplementary files}

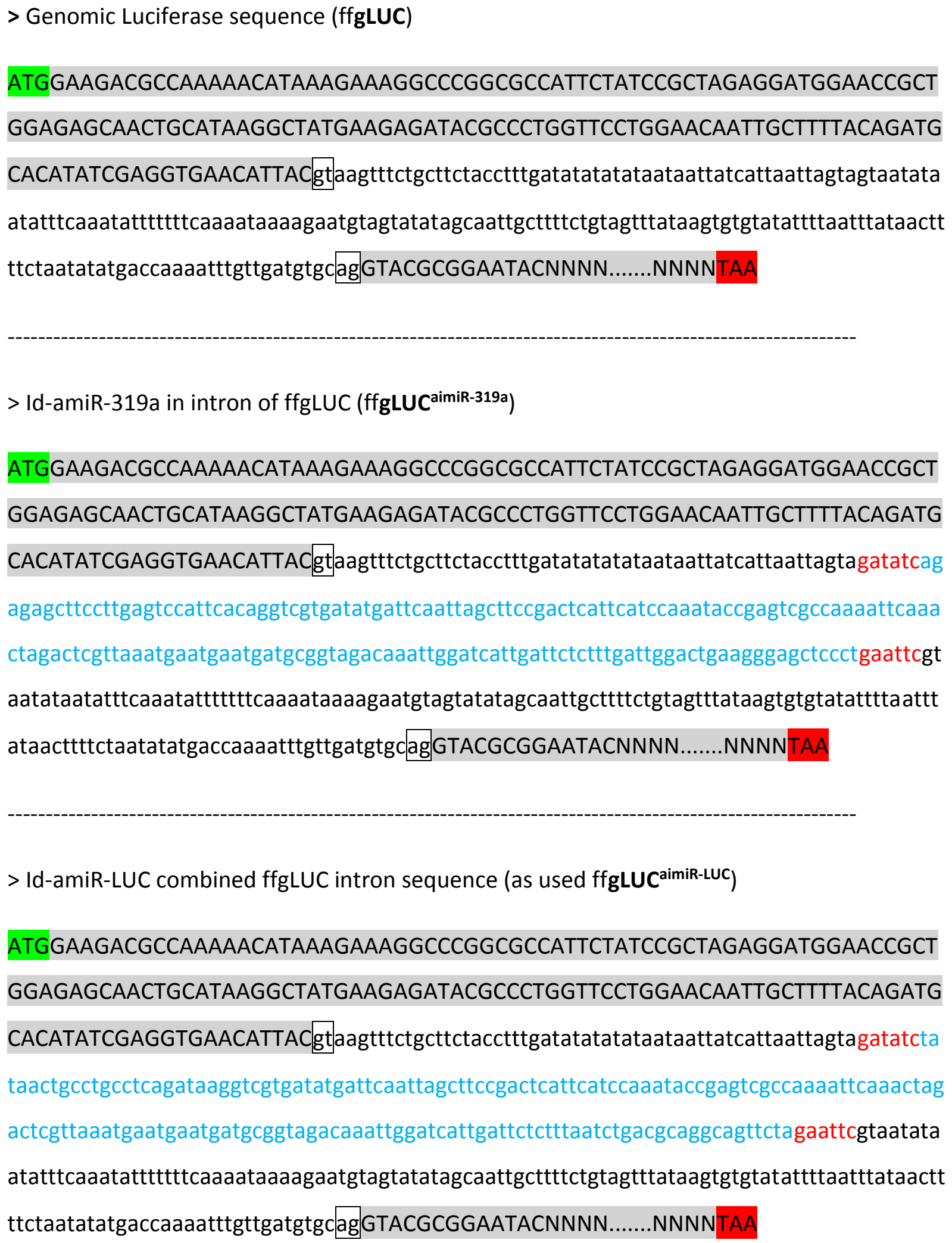
aatataatatttcaaatatttttttcaaaataaaagaatgtagtatatagcaattgcttttctgtagtttataagtgtgtatattttaattt ataacttttctaatatatgaccaaaatttgttgatgtgcagGTACGCGGAATACNNNN........NNNNTAA

> Id-amiR-LUC combined ffgLUC intron sequence (as used ffgLUC aimiR-LUC) $^{-}$

ATGGAAGACGCCAAAAACATAAAGAAAGGCCCGGCGCCATTCTATCCGCTAGAGGATGGAACCGCT GGAGAGCAACTGCATAAGGCTATGAAGAGATACGCCCTGGTTCCTGGAACAATTGCTTTTACAGATG CACATATCGAGGTGAACATTACgtaagtttctgcttctacctttgatatatatataataattatcattaattagtagatatcta taactgcctgcctcagataaggtcgtgatatgattcaattagcttccgactcattcatccaaataccgagtcgccaaaattcaaactag actcgttaaatgaatgaatgatgcggtagacaaattggatcattgattctctttaatctgacgcaggcagttctagaattcgtaatata atatttcaaatatttttttcaaaataaaagaatgtagtatatagcaattgcttttctgtagtttataagtgtgtatattttaatttataactt ttctaatatatgaccaaaatttgttgatgtgcag]GTACGCGGAATACNNNN........NNNNTAA 
> Id-amiR-PHYB combined ffgLUC intron sequence (as used ffgLUC aimiR-PHYB)

ATGGAAGACGCCAAAAACATAAAGAAAGGCCCGGCGCCATTCTATCCGCTAGAGGATGGAACCGCT GGAGAGCAACTGCATAAGGCTATGAAGAGATACGCCCTGGTTCCTGGAACAATTGCTTTTACAGATG CACATATCGAGGTGAACATTACgtaagtttctgcttctacctttgatatatatataataattatcattaattagtagatatcta gctgtaaaccgtaaggctcaggtcgtgatatgattcaattagcttccgactcattcatccaaataccgagtcgccaaaattcaaacta gactcgttaaatgaatgaatgatgcggtagacaaattggatcattgattctctttcagcctttcggtttacagttagaattcgtaatata atatttcaaatatttttttcaaaataaaagaatgtagtatatagcaattgcttttctgtagtttataagtgtgtatattttaatttataactt ttctaatatatgaccaaaatttgttgatgtgcagGTACGCGGAATACNNNN........NNNNTAA

$>$ Id-amiR319a in intron ffgLUC with deletion of intron sequence (ffgLUC del $^{\text {aimiR-319a }}$ )

ATGGAAGACGCCAAAAACATAAAGAAAGGCCCGGCGCCATTCTATCCGCTAGAGGATGGAACCGCT GGAGAGCAACTGCATAAGGCTATGAAGAGATACGCCCTGGTTCCTGGAACAATTGCTTTTACAGATG CACATATCGAGGTGAACATTACgtaagtttctgatatcagagagcttccttgagtccattcacaggtcgtgatatgattcaa ttagcttccgactcattcatccaaataccgagtcgccaaaattcaaactagactcgttaaatgaatgaatgatgcggtagacaaattg gatcattgattctctttgattggactgaagggagctccctgaattcaacttttctaatatatgaccaaaatttgttgatgtgcag GTAC GCGGAATACNNNN........NNNNTAA

Figure S1. Nucleotide sequences of all ff-gLUC/aimiRNA constructs used in this study. gatatc: - EcoR V site; gaattc: EcoR I site; Capital letter: exon sequences; small letters black: intron sequences; small letters blue: Id-amiRNA sequences based on miR319a. Specific sequences targeting TCP, LUC or PHYB are underlined. 5 'intron splice sequences are boxed (ag: 3'intron splice site, gt: 5'intron splice site) 
$>$ CDS Luciferase sequence (correct spliced)

ATGGAAGACGCCAAAAACATAAAGAAAGGCCCGGCGCCATTCTATCCGCTAGAGGATGGAACCGCT GGAGAGCAACTGCATAAGGCTATGAAGAGATACGCCCTGGTTCCTGGAACAATTGCTTTTACAGATG CACATATCGAGGTGAACATTACGTACGCGGAATACTTCGAAATGTCCGTTCGGTTGGCAGAAGCTAT GAAACGATATGGGCTGAATACAAATCACAGAATCGTCGTATGCAGTGAAAACTCTCTTCAATTCTTTA TGCCGGTGTTGGGCGCGTTATTTATCGGAGTTGCAGTTGCGCCCGCGAACGACATTTATAATGAACG TGAATTGCTCAACAGNNNNNNNNNNNNNNNNNNNNNNNNNNNNNNNNNNNNNNNNNNNNN NNNNNNNNNNNNNNNNNNNNNNNNNNNNNNNNNNNNNN

> shorter CDS Luciferase sequence (3'splice site shifted)

ATGGAAGACGCCAAAAACATAAAGAAAGGCCCGGCGCCATTCTATCCGCTAGAGGATGGAACCGCT GGAGAGCAACTGCATAAGGCTATGAAGAGATACGCCCTGGTTCCTGGAACAATTGCTTTTACAGATG CACATATCGAGGTGAACATTACAGCTATGAAACGATATGGGCTGAATACAAATCACAGAATCGTCGT ATGCAGTGAAAACTCTCTTCAATTCTTTATGCCGGTGTTGGGCGCGTTATTTATCGGAGTTGCAGTTG CGCCCGCGAACGACATTTATAATGAACGTGAATTGCTCAACAGNNNNNNNNNNNNNNNNNNNNN NNNNNNNNNNNNNNNNNNNNNNNNNNNNNN

> shorter CDS Luciferase sequence (5'and 3'splice site shifted)

ATGGAAGACGCCAAAAACATAAAGAAAGGCCCGGCGCCATTCTATCCGCTAGAGGATGGAACCGCT GGAGAGCAACTGCATAAGGCTATGAAGAGATACGCCCTGGTTCCTGGAACAATTGCTTTTACAGATG CACATATCGAGGAGCTATGAAACGATATGGGCTGAATACAAATCACAGAATCGTCGTATGCAGTGAA AACTCTCTTCAATTCTTTATGCCGGTGTTGGGCGCGTTATTTATCGGAGTTGCAGTTGCGCCCGCGAA CGACATTTATAATGAACGTGAATTGCTCAACAGNNNNNNNNNNNNNNNNNNNNNNNNNNNNNN NNNNNNNNNNNNNNNNNNNNNNNNNNNNNNNN

Figure S2. RT-PCR products sequence from ffgLUC del $^{\text {aimiR-319a }}$ transgenic plant. 


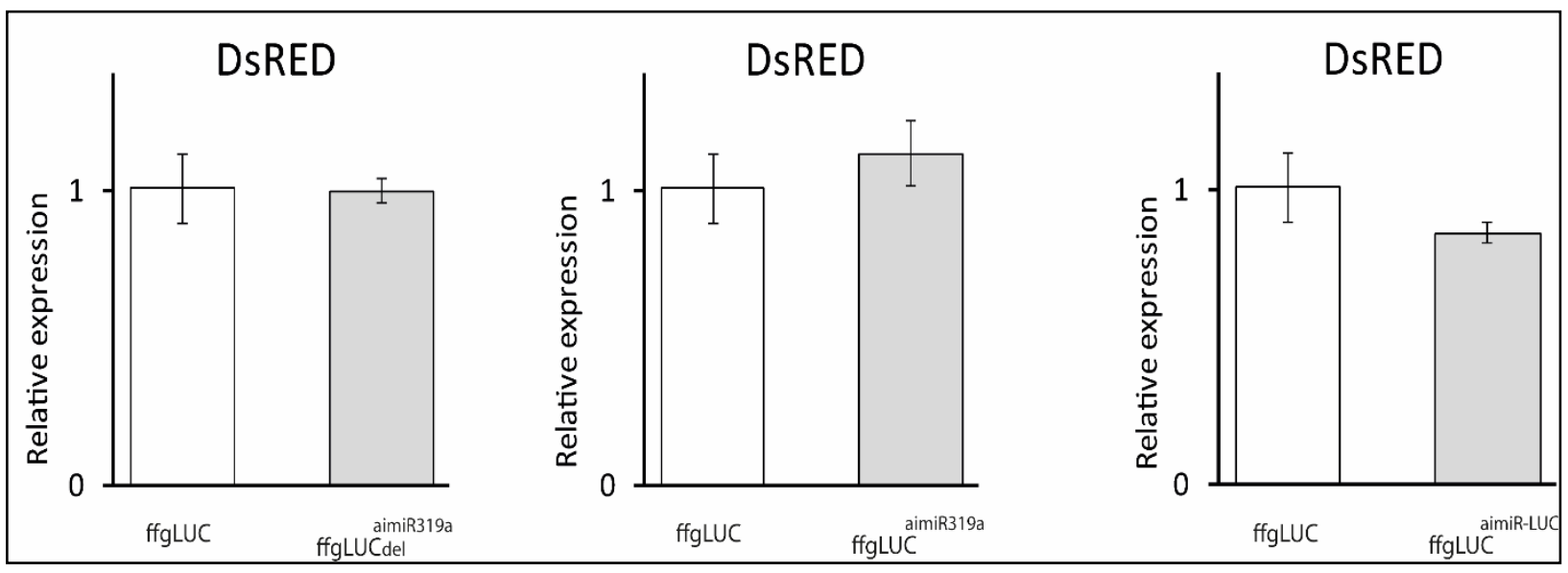

Figure S3. A similar transformation efficiency were confirmed by quantify the DsRED gene expression in transient assay samples. The quantification data is normalized against the $N$.benthamiana reference with UBI3 as internal control. Error bars represent standard error. 


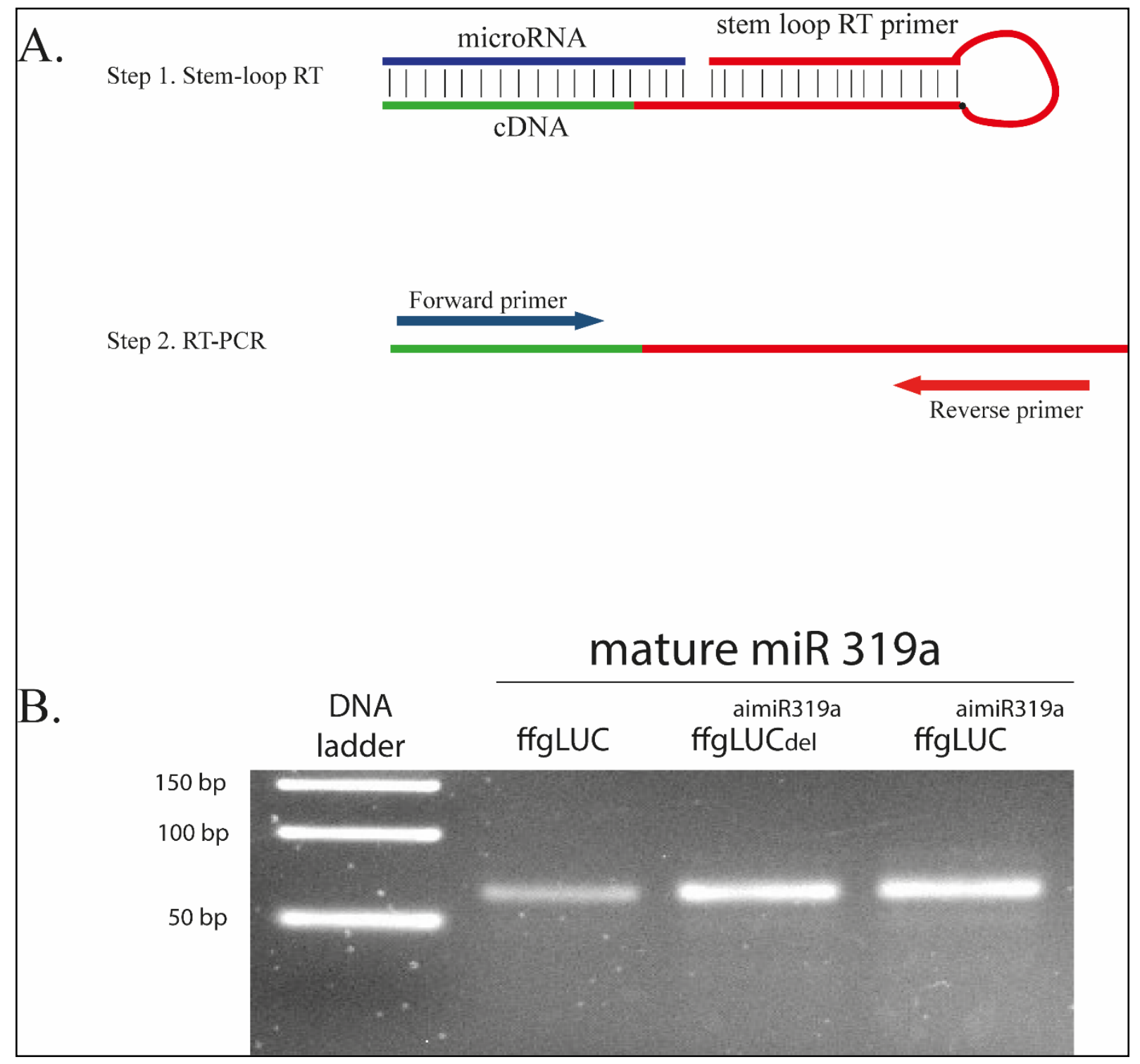

Figure S4. Confirmation of mature aimiR-319a expression in stem-loop RT-PCR assay. Illustration of RT-PCR method for amplification of mature microRNA (A). Gel electrophoresis results from stem-loop RT (B). ffgLUC plant used as a positive control for endogenous mature miR319a. A mature microRNA specific forward primer and universal reverse primer were used for PCR amplification. Mature microRNA products were obtained using 25 cycling of RT-PCR and analysed on $4 \%$ agarose gel in 1xTAE. Predicted products were compared with 50bp DNA ladder. 


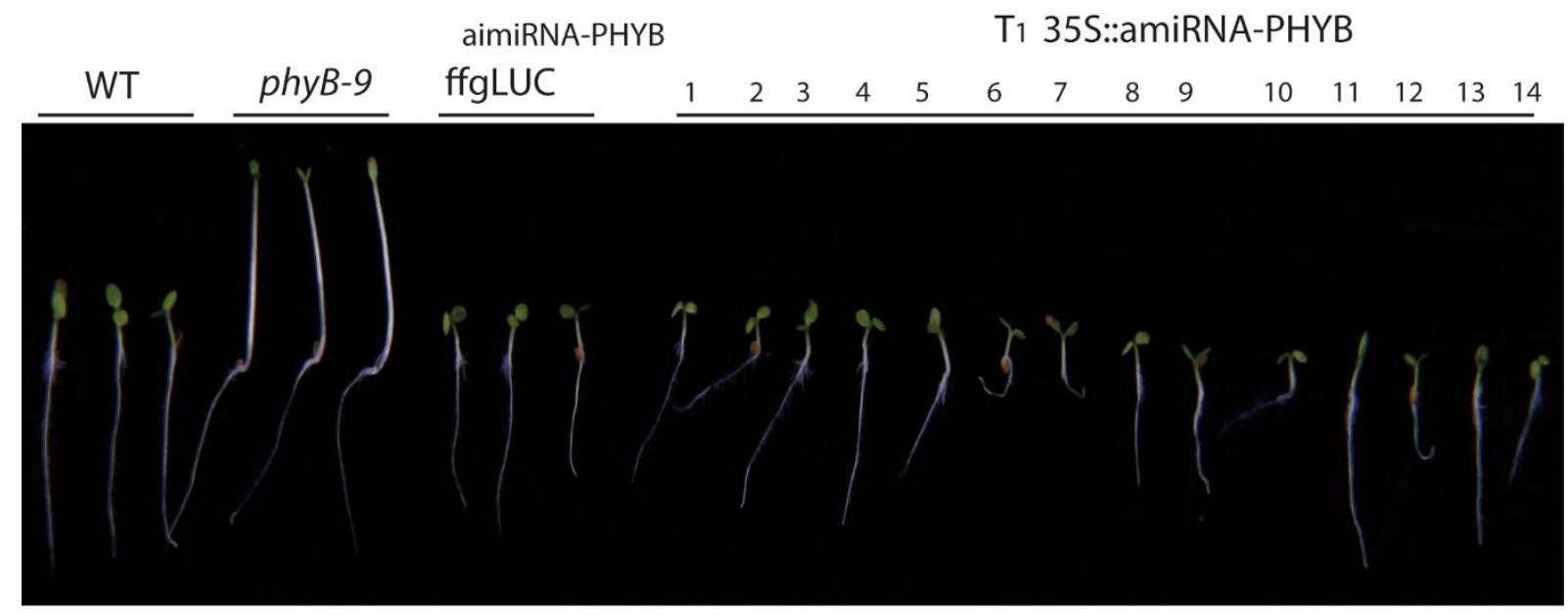

Figure S5. PHYB silencing phenotype under constant cR. 


\begin{tabular}{|l|l|l|l|l|l|l|}
\hline Transformation & & $\begin{array}{l}\text { in } \\
\text { seeds }\end{array}$ & $\begin{array}{l}\text { Positive } \\
\text { tested }\end{array}$ & $\begin{array}{l}\text { transformants } \\
\text { expression }\end{array}$ & $\begin{array}{l}\text { LUC } \\
\text { leaf } \\
\text { phenotype }\end{array}$ & NHYB \\
35S::ffgLUC & NA & NA & NA & NA & NA & NA \\
\hline 35S::ffgLUCdel aimiR-319a & $>25$ & 17 & $17 *$ & 17 & NA & NA \\
\hline 35S::ffgLUC & $>25$ & 19 & 19 & 17 (2 dead) & NA & NA \\
\hline 35S::ffgLUC-319a & $>20$ & 20 & $20 * *$ & NA & 20 & NA \\
\hline 35S::ffgLUC & $>12$ & 10 & 10 & NA & NA & \\
\hline 35S::amiRiR-LUC & $>19$ & 19 & $19 * *$ & NA & 19 & NA \\
\hline
\end{tabular}

Table S1. Stable transformation of Arabidopsis WT or Arabidopsis line ff-gLUC-1 with the different expression constructs. NA: not applicable, * very low LUC activity; ${ }^{* *}$ low LUC activity. 


\begin{tabular}{|c|c|c|c|}
\hline PRIMER NAME & SEQUENCE & USED FOR & REF \\
\hline Luc-F-Ncol & agcaaaccatggaagacgccaaaaac & cloning of full LUC gene & This study \\
\hline Luc-R-Notl & $\underline{\text { agcaaagcggccgcttacaatttggactttccgccctt }}$ & cloning of full LUC gene & This study \\
\hline Luc-intron-R-EcoR V & $\underline{\text { aaagatatctactaattaatgataattatt }}$ & cloning of first exon+ intron LUC & This study \\
\hline Luc-intron-F-EcoR I & $\underline{\text { aaagaattcgtaatataatatttcaaatatttttttcaaaataa }}$ & cloning of intron + second exon LUC & This study \\
\hline miR319a_F-EcoR V & $\underline{\text { aaagatatcagagagcttccttgagtccattcac }}$ & cloning of miRNA319a & (Liang et al., 2012) \\
\hline miR319a_R-EcoRI & $\underline{\text { tttgaattcagggagctcccttcagtccaatc }}$ & cloning of miRNA319a & (Liang et al., 2012) \\
\hline AmiRphyB-F-EcoR V & $\underline{\text { aaagatatctagctgtaaaccgtaaggctcaggtcgtgatatgattca }}$ & cloning of artificial miR-PHYB & This study \\
\hline AmiRphyB-R-EcoR I & aaagaattctaactgtaaaccgaaaggctgaaagagaatcaatgatcca & cloning of artificial miR-PHYB & This study \\
\hline AmiRluc-F-EcoR V & $\underline{\text { aaagatatctataactgcctgcctcagataaggtcgtgatatgattca }}$ & cloning of artificial miR-LUC & This study \\
\hline AmiRluc-R-EcoR I & $\underline{\text { aaagaattctagaactgcctgcgtcagattaaagagaatcaatgatcca }}$ & cloning of artificial miR-LUC & This study \\
\hline Luc splice 2_R-EcoR V & $\underline{\text { aaagatatcagaaacttacgtaatgttcacctcg }}$ & cloning of first exon + intron splice LUC & This study \\
\hline Luc splice 2_F-EcoR I & $\underline{\text { aaagaattcaacttttctaatatatgaccaaaatttgtt }}$ & cloning of intron splice + second exon LUC & This study \\
\hline AthPHYB_F & cgttgggtgttgctcctagt & qPCR in A.thaliana (At2g18790) & This study \\
\hline AthPHYB_R & gataccccgcatcgcctaaa & qPCR in A.thaliana (At2g18790) & This study \\
\hline AthTCP2_F & aacggcggagcattcaatctt & qPCR in A.thaliana (At4 g18390) & (Nag et al., 2009) \\
\hline AthTCP2_R & gcctttacccttatgttctga & qPCR in A.thaliana (At4 g18390) & (Nag et al., 2009) \\
\hline AthTCP3_F & catccagtttatagccaaa & qPCR in A.thaliana (At1 g53230) & (Nag et al., 2009) \\
\hline AthTCP3_R & atggcgagaatcggatgaa & qPCR in A.thaliana (At1 g53230) & (Nag et al., 2009) \\
\hline AthTCP4_F & ccttcaacgacgtcgtttcagccag & qPCR in A.thaliana (At3 g15030) & (Nag et al., 2009) \\
\hline AthTCP4_R & gtgaaccggtggaggaaggtgatg & qPCR in A.thaliana (At3 g15030) & (Nag et al., 2009) \\
\hline $\mathrm{NbTCP} 4$ _F & ctgcatctgctgcaaacatt & $\begin{array}{l}\text { qPCR in N.benthamiana } \\
\text { (Niben101Scf01002g02011.1) }\end{array}$ & This study \\
\hline $\mathrm{NbTCP} 4 \_\mathrm{R}$ & aacccattgggaaaaaggac & $\begin{array}{l}\text { qPCR in N.benthamiana } \\
\text { (Niben101Scf01002g02011.1) }\end{array}$ & This study \\
\hline AthActin F & ggtaacattgtgctcagtggtgg & qPCR in A.thaliana & This study \\
\hline AthActin $\mathrm{R}$ & aacgaccttaatcttcatgctgc & qPCR in A.thaliana & This study \\
\hline NbUbi3_F & gccgactacaacatccagaagg & qPCR in N.benthamiana & This study \\
\hline NbUbi3_R & tgcaacacagcgagcttaacc & qPCR in N.benthamiana & This study \\
\hline DsRED F & gaagctgaaagacggtggtc & qPCR in N.benthamiana & This study \\
\hline DsRED R & cgtccctcggttctttcata & qPCR in N.benthamiana & This study \\
\hline LUC-RT-F & cgaggtgaacattacgtaagtttc & RT-PCR & This study \\
\hline LUC-RT-R & gtattccgcgtacctgcac & RT-PCR & This study \\
\hline MIR-319A RT & gtcgtatccagtgcagggtccgaggtattcgcactggatacgacagggag & RT-PCR & This study \\
\hline MIR-319A F & cggcggttggactgaagggag & RT-PCR & This study \\
\hline AMIRPHYB RT & gtcgtatccagtgcagggtccgaggtattcgcactggatacgactaactg & RT-PCR & This study \\
\hline AMIRPHYB F & cggcggcagcctttcggttta & RT-PCR & This study \\
\hline UNIVERSAL REVERSE & gtgcagggtccgaggt & RT-PCR & $\begin{array}{l}\text { (Varkonyi-Gasic et } \\
\text { al., 2007) }\end{array}$ \\
\hline
\end{tabular}

Table-S2. List of primers were used in this work. 



\section{CHAPTER-6}

General Discussion 


\section{The need for a scientific basis for plant growth control in horticulture}

The on-going climate changes are predicted to have a negative impact on crop yields in agriculture and therefore a negative impact on food security. This is happening at a time when the increase in the global population is raising food demand. Food production in greenhouses, with in-door climate control, may be part of the solution to secure food demand. Indeed, the coming decades the focus could be to improve crop production in both small and large scale greenhouse farming. Already in many countries greenhouse farming is at an industrial scale, growing the maximum amount of crop foods at a minimum price. However, at the same time, these efforts need to keep a friendly relationship with the social or environmental consequences. Farming output at an industrial level requires a scientific basic understanding of the regulation of plant growth in order to apply the most effective growth control measures. Indeed, fundamental research can help to improve crop quality, yield and resilience against biotic stresses induced by different pathogens.

One option for control of plant growth in greenhouses is the use of chemical growth regulators, which is not environmentally friendly. As an alternative to chemical treatment for inhibiting of plant growth in horticulture, nowadays light/temperature regimes named -DIF are used. This is a more sustainable solution compared to the use of chemical growth inhibitors. In -DIF condition, plants are exposed to cold day and warm night instead of cold night and warm day (+DIF) (Carvalho et al., 2002). This results in more compact plants due to reduced elongation (Stavang et al., 2005). Understanding the molecular basis of growth control under -DIF and translating these insights into new or improved protocols to control plant growth has been the aim of the STW project 'Compact Plants' (13149). Within this project, my own research and that of PhD candidate Mark van Hoogdalem focused on understanding the light and temperature regulation of a specific subset of transcription factors involved in elongation (PIFs) or suppression of elongation (HY5). Previous results had shown that the activity of PIF4 is limited under -DIF, resulting in reduced auxin and ethylene signaling (Franklin et al., 2011; Kunihiro et al., 2011) and eventually reduced PIF3 activity which regulates downstream elongation responses (Bours et al., 2013), The action of PIFs in the light is strongly influenced by phytochromes (Li et al., 2011; Jung et al., 2016; Legris et al., 2016) and this was the motivation to get a better understanding of the transcriptional 
regulation of phytochrome genes. This work resulted in improved insight in how PHY genes influence each other's transcriptional activity depending on the light conditions and as function of development. In this research we also discovered the strong induction of PHYA and PHYB expression under FR light, which may have its application in steering plant growth once understood better (Chapter 2).

\section{Things are different at warm temperature}

Plant growth and morphology is altered under warm ambient temperature. The morphological response of plants to warmth is different from changes induced by actual heat stress, during which protein folding and function may be compromised. The specific growth response to warm ambient temperature is called thermo-morphogenesis (Quint et al., 2016), and understanding thermo-morphogenesis is just another aspect of understanding general growth regulation in plants. Thermomorphogenesis simultaneously affects plant growth (positive effect) and plant resilience (negative effect) and PIF4 and BZR1 function at the molecular switch that can steer the plant in these two different modes (Gangappa et al., 2017; Martínez et al., 2018). Recent research indicates that thermo-morphogenesis is accompanied by chromatin modification at specific gene loci that affect gene regulation (Tasset et al., 2018), photoreceptor protein activity (PHYB) (Jung et al., 2016; Legris et al., 2016) or auxin biosynthesis and other phytohormone signalling (Ibañez et al., 2018). In this thesis we discovered how MED25 may play a role in the epigenetic changes that mediate these warm temperature responses by demonstrating that MED25 binds to PIF4 and BZR1 and that MED25 is required for normal transcriptional activity of PIF4.

\section{Complex regulation PIF4}

Besides understanding the transcriptional regulation of PHY genes, my thesis research was also about understanding the regulation of PIF4 and PIF4 transcriptional activity. Multiple studies have been shown that PIF4 acts as a hub in plant elongation responses and immunity (Koini et al., 2009; Nomoto et al., 2012; Choi and Oh, 2016; Gangappa et al., 2017). Most 
studies focus on the regulation of downstream targets of PIF4. However, we felt that in order to understand regulation of PIF4 target genes we also need to understand the regulation of the PIF4 gene itself. PIF4 protein activity is the combined function of PIF4 gene transcription, PIF4 mRNA translation, PIF4 protein activation and PIF4 protein stability. In this thesis work we especially aimed for a better understanding of the transcriptional regulation of the PIF4 gene itself (Chapter 3). In addition, in Chapter 4 we investigated the transcriptional activity of PIF4 protein in combination with the Mediator component MED25. These two studies are linked by the fact that transcriptional activity of PIF4 protein with MED25 is also part of the transcriptional regulation of the PIF4 gene itself. There are no studies on the role of PIF4 on its own PIF4 gene expression. Regulation of PIF4 gene transcription is complicated by the many factors that either affect PIF4 transcriptional activity or PIF4 protein stability. In addition, many of these factors are under direct or indirect control of PIF4 itself, resulting in a very complex feedback regulation that seems to keep tight control on PIF4 activity under different environmental conditions and different genetic backgrounds. Each study on PIF4 activity reveals only part of the total puzzle, and integration of all these parts into a full picture is still not easy. However, by investigating the expression of PIF4 in a certain mutant or in response to a given environmental signal, new aspects of PIF4 gene regulation can be revealed.

\section{Integrating results into an updated growth model}

The work in this thesis mostly relates to understanding the transcriptional regulation of PIF4 and to understanding the transcriptional activity of PIF4, because PIF4 is a key transcription factor in growth responses of Arabidopsis. This thesis research started with the simplified growth model shown in Chapter 1, which depicts the key upstream position of PIF4 and downstream position of PIF3 in regulating growth genes. It also depicts the role of phytochrome on the action of PIF4 and PIF3, although more transcription factors besides PIF4 are involved in regulating growth. Indeed, the action of PIF4 and PIF5 seem to be closely related (Sun et al., 2013). Moreover, PIF4 acts together with BZR1 (Oh et al., 2012) and PIF4 and BZR1 are now recognized as two key factors in growth responses. Also BZR1 is not acting alone, as the close homolog of BZR1, BES1 is shown to have very similar activity as BZR1 (Martínez et al., 2018; Wu et al., 2018). Recent insights show that, because of the intimate 
way PIF4 and BZR1/BES1 influence each other's activity, their actions cannot be viewed separately (Martínez et al., 2018). In the four years of this thesis work, 117 additional papers on PIF4, 49 papers on PIF5, 99 papers on BZR1 and 69 papers on BES1 have been published, illustrating the importance of these transcription factors in control of plant growth. Moreover, recent papers also describe the role of PIF4 and BZR1 in the trade-off between growth and resistance (Gangappa et al., 2017) and the role of PIF4 and BZR1 in thermomorphogenesis (Ibañez et al., 2018). This provides additional motivation to understand the transcriptional regulation of the PIF4 and BZR1 genes themselves and to understand how the PIF4 and BZR1 protein regulate transcription of different target genes. As the name implies (Phytochrome Interacting Factors) the PIF proteins interact with light activated phytochromes in the nucleus, which leads to their proteasome mediated destruction. Thus, the level of active phytochrome is an important determinant of PIF4 activity. The level of phytochrome protein is initially determined by transcription of the phytochrome genes. Therefore, also understanding phytochrome gene transcription is an integral part of understanding the actions of PIF4. In this thesis we focussed on the transcriptional regulation of the phytochrome genes (Chapter 2) and the PIF4 gene itself (Chapter 3). Here, I will describe how the results presented in this thesis and recent published research can be integrated into an updated model of growth control (Figure 1).

A key finding published during our research, is the central role of BZR1 in transcriptional control of PIF4, especially under warmth (Ibañez et al., 2018). BZR1 is a positive regulator of PIF4 but activity of BZR1 is indirectly coupled to the action of PIF4 through a feedback loop involving brassinosteroid (BR) biosynthesis and signalling (Ibañez et al., 2018; Martínez et al., 2018). The PIF4 activated BR signalling inhibits the kinase BIN2 which leads to further activation of BZR1 but also to further activity of PIF4, as phosphorylation of both BZR1 and PIF4 by BIN2 leads to destabilisation of these two transcription factors. We have shown that this positive feedback regulation of PIF4 is kept under control by a negative feedback regulation at the transcription level by PIF4 protein itself, as PIF4 is a negative transcription factor for its own promoter activity (Chapter $\mathbf{3}$ ). In addition we have shown that PIF4 can stimulate transcription of PHYB (Chapter 2,3 ), thus PIF4 protein stimulates the activity of a component that limits PIF4 protein stability, providing an additional negative feedback on PIF4 activity. Recently it was shown that transcription of PHYA is under control of PIF4 and PIF5. 
PHYA interacts with PIF3 (which is downstream in the model) leading to its destabilisation. Thus, PIF4 (which is upstream in the model; Figure 1) may indirectly negatively affect PIF3 activity. As PIF3 is more directly linked to activation of growth genes, stimulation of upstream PIF4 in the light may therefore be limited by increased activity of PHYA on downstream PIF3.

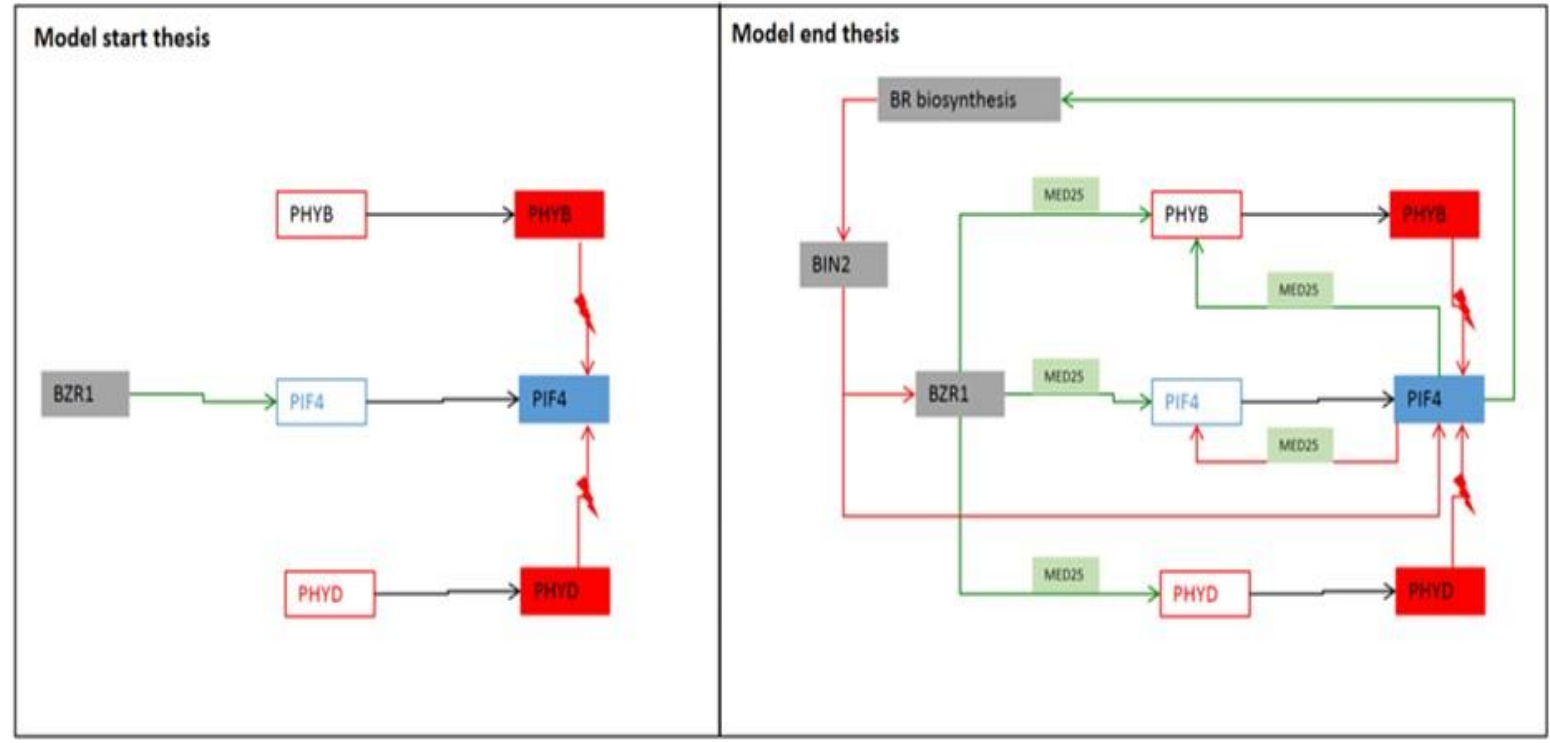

Figure 1. Model PIF4 regulation at start and end of thesis: feedback at PIF4 locus by PIFs, PHYs, MED25 and BR, BIN2, BZR1. open boxes: genes, closed boxes: proteins. black arrows: transcription, red arrows: negative interaction, green arrows: positive interaction. red lightning bolt: light activated phytochrome.

The updated interaction model (Figure 2 ) explains why PIF4 gene expression is a complex function of light: in the light PIF4 protein destabilised by activated PHYB, resulting in less negative feedback on its own gene expression. However, reduced PIF4 activity in the light also leads to reduced PHYB expression, which has a positive effect on PIF4 protein stability. At the same time, reduced PIF4 protein activity reduces the BR biosynthesis and signalling and therefore BIN2 action, resulting in an opposite effect on PIF4 stability. We give two examples of how difficult it is to predict PIF4 expression in different mutant backgrounds: with a negative feedback on its own promoter activity initial prediction would be that PIF4 transcription is higher in a pif4-2 mutant background, and with the positive action of BZR1 on PIF4 promoter activity the initial prediction was that PIF4 expression is higher in bzr1-1D. Both simple predictions are wrong: in both mutants PIF4 promoter activity is reduced, which can be explained by stronger effects of PIF4 in the different indirect feedback loops acting on its 
own promoter activity (see discussion Chapter 3). Therefore, it seems that the more we learn about the regulation of PIF4 gene transcription, the more complex it becomes to make predictions about its actual regulation without the aid of computer modelling. With such models it may become possible to predict the outcome of short term light treatments for optimized PIF4 and PIF3 control and related control of downstream growth genes.

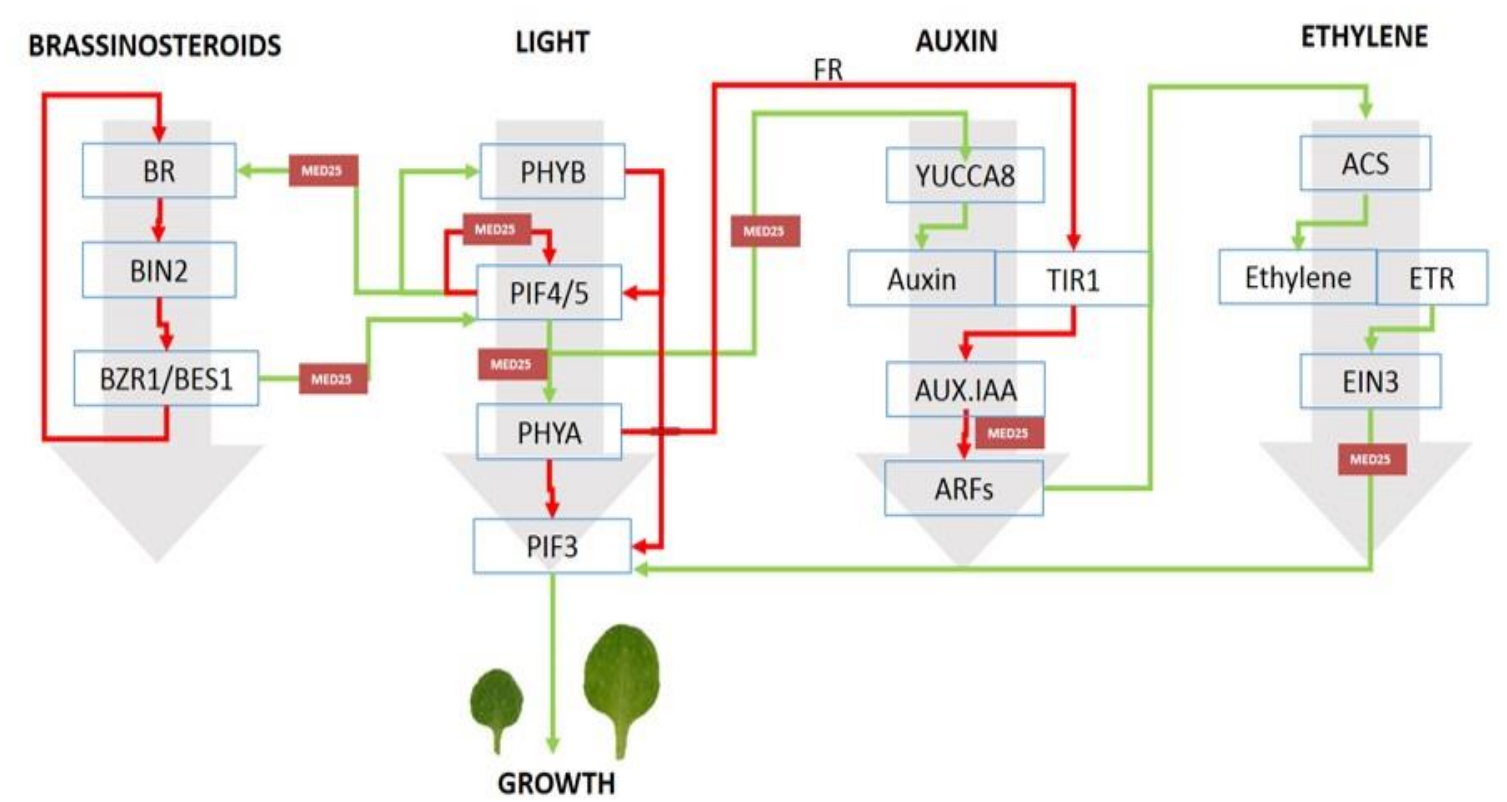

Figure 2. Signal transduction pathway towards elongation as elucidated near the end of this project. Red arrows: negative interaction; Green arrows: positive interaction; Grey arrows: different signal transduction pathways. The different positions where MED25 interacts with transcription factors are indicated in red blocks with MED25. The role of MED25 for PIF3 is speculative at this moment.

\section{FR High Irradiance transcriptional responses for PHYB and PHYA}

In Chapter 2, I describe the regulation of PHY genes, making use of pPHY:LUC reporter plants. The interactions that we uncovered in this research may need further validation by endogenous PHY gene expression analysis for all the interactions we uncovered. However, the main novel observations that were uncovered with the use of the PPHY:LUC reporter plants were validated by qPCR analysis of the respective endogenous PHY genes. For instance, the qPCR analysis confirmed the FR induction of PHYA and PHYB, it confirmed for PHYB that the induction of PHYB expression under FR is not a function of PHYA, that expression of PHYA is 
suppressed by PHYD and that upregulation of PHYA by FR requires PHYE. The strong and acute induction of pPHYB:LUC by FR could make the PHYB promoter an attractive tool for (FR) light induced expression in plants, for instance for proteins whose activity have a negative impact on plant survival like some of the terpene synthases that can produce useful products in plants. However, for such potential application it first needs to be tested whether the default low expression of a transgene under the PHYB promoter is low enough to avoid deleterious effects on plant growth.

Our results indicate that for the FR light treatments PIF4 is required for the transcriptional response of PHYB. PIF4 was already identified as regulator of PHYA expression and future analysis will have to show whether the induction of PHYA under FR is also dependent on PIF4. However, we note that the expression profile of PIF4 under FR closely matches the expression profile of PHYA under FR, suggesting a direct regulation of PHYA by PIF4 under FR. The strong direct induction of PHYB does not match the expression profile of PIF4 and suggests other factors may be involved in the direct transcriptional response of PHYB under FR. Also the putative role of PIF4 in PHYA expression under FR is not entirely in line with our findings that PIF4 represses PIF4 expression. Such a mechanism should limit large changes in PIF4 expression. The alternative explanation could be that negative feedback of PIF4 on its own promoter is conditional, as is described for the action of PIF4 at the YUCCA8 promoter (vd Woude 2018 under review). For the PIF4 target gene YUCCA8 the change in PIF4 activity at normal temperature and under warmth is related to a change in histone modifications and histone exchange at the YUCCA8 promoter (vd Woude 2018 under review). We have shown that MED25 is involved in this switch of histones activity by demonstrating that MED25 binds to HDA9 and that MED25 destabilizes HDA9 (Chapter 4). It still needs further investigation whether a similar regulation occurs at the PIF4 promoter itself. However, CHIP analysis with HDA9 does indeed identify PIF4 as target (Chen et al., 2016). Moreover, similar questions can be raised about the strong upregulation of PIF4 expression under FR, especially since the expectations are the PIF4 protein is stabilized under FR. Therefore, further research is needed to determine whether the upregulation of PIF4 under FR involves histone modifications at the PIF4 locus and what the potential role of MED25 is in this. Indirect evidence that MED25 is important for the negative feedback regulation of PIF4 on its own promoter activity comes 
from the observation of higher PIF4 expression in pft1-2 at normal temperature and the even stronger upregulation of PIF4 expression in pft1-2 under warmth (Chapter 4).

\section{Confusion about the effect of PIF4 overexpression}

In this research, I have made use of firefly luciferase reporter genes to test promoter activity in stable transformed plants, or to test promoter activity in transient expression assays in N.benthamiana leaves for evaluating the contribution of transcription factors to the expression of the LUC reporter. The measurement of LUC activity in stable transformed plants or in leaves of N.benthamiana using LUMINATOR is easy and versatile, as promoter activity can be monitored as function of both light quality and temperature. However, we also encountered some problems with the interpretation of LUC reporter activities, especially in transient expression assays using PIF4 target gene reporters and PIF4 effector genes. In addition, the effect of PIF4 overexpression in our results was often different from what is reported in literature. While some issues still need to be resolved, the seemingly contradictory results with PIF4 could eventually be explained by the fact that we use untagged PIF4 in overexpression experiments, while in many published papers a tagged version of PIF4 was used. Here, I discuss the discrepancies between our results compared to published results:

(1) different results of PIF4 effector construct in transient assays with PIF4 target genes: the tag on PIF4 is affecting PIF4 activity in transient assays.

(2) difference between in planta and leaf-extract LUC activity: for PIF4 overexpression in leaf cells lower efficiency of the LUC reaction, e.g. due to competition for ATP?

(3) different effect for PIF4OE on hypocotyl elongation: examples used in literature about PIF4OE stimulating hypocotyl elongation is the exception rather than the rule.

(4) Higher PIF4 expression in the MED25 mutant pft1-2 does not link to increased hypocotyl elongation: in pft1-2 the PIF4 expression is uncoupled from downstream PIF4 target gene expression.

Different results with untagged and tagged PIF4 in plant transient expression assays. 
During tests of the role of PIF4 in the activation of PIF4 target genes (YUCCA8, IAA29) in transient expression assays by agro-infiltration of N.benthamiana leaves, we found that our own results are different from published results. Several groups have used the transient expression system in N.benthamiana leaves to demonstrate to positive action of PIF4 on YUCCA8. PIF4 binds to the G-box present in the YUCCA8 promoter but not to G-box present in promoter of YUCCA5/9/10 (Sun et al., 2012). A PIF4 effector construct was used to demonstrate that PIF4 activates the pYUCCA8:LUC reporter in a transient expression assay in N.benthamiana leaves (Sun et al., 2012). We repeated this experiment with our own PIF4 effector gene. When co-expressed with the reporter YUCCA8-LUC, the PIF4 effector construct resulted in lower LUC activity in the agro-infiltrated leaf instead of higher activity as shown by Sun et al., (Sun et al., 2012). We ascribe this discrepancy in results to differences in the PIF4 effector construct: according to methods in Sun et al., (Sun et al., 2012), their PIF4 coding fragment for the effector construct was amplified by PCR with the forward primer 5CACCATGGAACACCAAGGTTGGAG-3 and reverse primer 5-GTGGTCCAAACGAGAACCGT-3 (Sun et al., 2012). This reverse primer used for the PIF4 amplification does not contain a stop codon, suggesting that their effector gene expresses a PIF4 protein with an unknown extension at the C-terminus (35S:PIF4-x). In contrast, our own PIF4 effector construct expresses PIF4 without C-terminal extension (35S:PIF4).

Moreover, the studies on the regulation of BR biosynthesis genes by Martinez et al., (Martínez et al., 2018) presented us with a similar discrepancy. In these studies a PIF4 effector construct is used, expressing a tagged version of PIF4 (35S:PIF4-HA) in combination with the PIF4 target reporter pPIL1:LUC. In their assay, the pPIL1:LUC activity was quantified in intact N.benthamiana leaf discs and these assays show a very strong stimulation of pPIL1 promoter activity by PIF4-HA. In contrast, in a similar assay with pPIL1:LUC and our own PIF4 effector gene (without C-terminal tag) the pPIL1:LUC activity is suppressed in N.benthamiana leaves (data not shown). Combined, this leads us to speculate/propose that untagged PIF4 and Cterminal tagged PIF4 do not behave the same in transient expression assays for PIF4 target reporter genes: untagged PIF4 leads to suppression of PIF4-target reporter genes, while tagged PIF4 leads to stimulation of PIF4-target reporter genes. Thus, we tested the untagged PIF4 effector (35S:PIF4) and two PIF4 effector constructs with a C-terminal tag: 35S:cLUC-PIF4, expressing PIF4 with a C-terminal half cLUC extension, or 35S:PIF4-nLUC, expressing PIF4 
protein with a C-terminal half nLUC extension. These half-LUC proteins do not have any LUC activity by themselves. The different PIF4 effector constructs were tested with our pIAA29:LUC reporter. The IAA29 gene is induced by PIFs and PIF4 binds to the promoter of IAA29 (Hornitschek et al., 2009; Hornitschek et al., 2012). The pIAA29:LUC was co-expressed with different PIF4 effector constructs. When LUC activity is imaged in infiltrated N.benthamiana leaves, results show that the PIF4 effector without extension reduces LUC activity from the pIAA29:LUC compared to control pIAA29:LUC+EV (Figure 3). In contrast, the PIF4 effectors with a C-terminal or N-terminal extension show increased activity for the pIAA29:LUC reporter in leaves (Figure 3). This shows that the C-terminal or N-terminal tagged PIF4 proteins are active, but their activity is different from the untagged PIF4 protein in N.benthamiana leaves.

A)

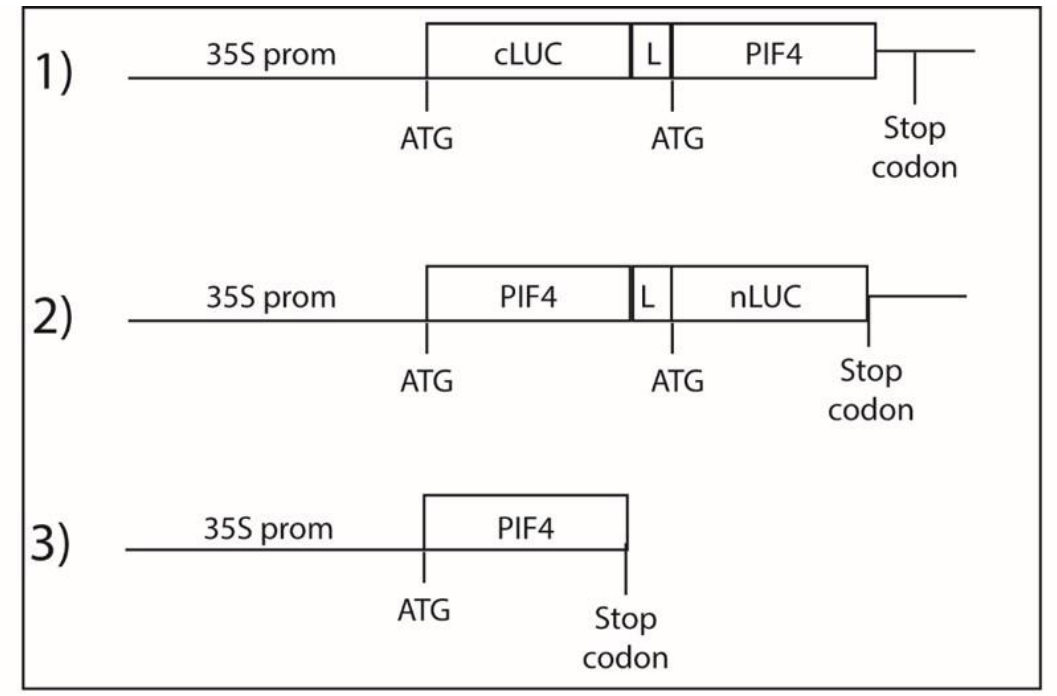

B)

\begin{tabular}{|c|c|}
\hline $\begin{array}{c}\text { pIAA29:LUC } \\
+ \text { EV }\end{array}$ & $\begin{array}{c}\text { PIAA29:LUC } \\
+ \text { CLUC-PIF4 }\end{array}$ \\
\hline $\begin{array}{c}\text { PIAA29:LUC } \\
+ \text { PIF4 }\end{array}$ & $\begin{array}{c}\text { pIAA29:LUC } \\
+ \text { PIF4-nLUC }\end{array}$ \\
\hline
\end{tabular}

C)

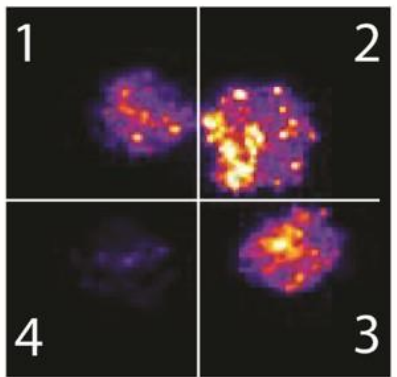

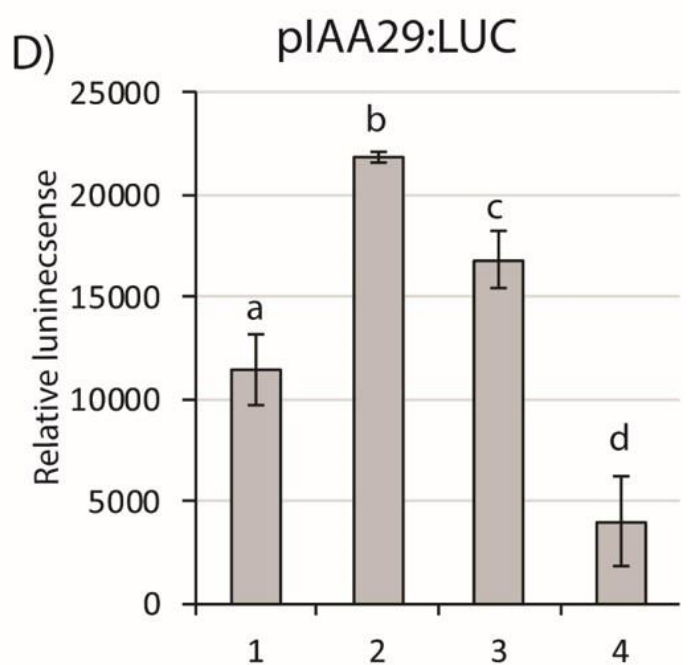


Figure-3. Effect of TAG to PIF4 function on pIAA29:LUC co-expression in N.benthamiana transient assay. (A). Three type of constructs were created for PIF4 function. 1) cLUC fused PIF4 (without own stop codon); 2) PIF4 (without stop codon) fused with nLUC; 3) PIF4 with own stop codon. All three construct derived with 355 promoter. (B, C, D). The pIAA29:LUC reporter was co-expressed in N.benthamiana leaves with Empty Vector (EV) and effectors CLUC-PIF4-x, PIF4-nLUC or PIF4 without tag. An average value of each samples is based on six individual leaves. Bars with a different letter $(a, b, c, d)$ show significant differences ( $p$-value<0.05).

\section{Different LUC activity in in planta and in leaf extract assays?}

The transient expression of the LUC reporter with different effectors can be assayed in intact leaves with LUMINATOR to obtain an image of the in planta LUC activity or results can be obtained in an extract of $N$.benthamiana leaves by measuring LUC activity in an in vitro extract assay. Leaf imaging of pYUCCA8:LUC resulted in reduction in luminescence with PIF4 coexpression compared to EV control (Figure 4A). In the leaf extract assay the variation in agroinfiltration can be corrected by calibration with a 35S-renillaLUC control construct. When the PIF4 effector construct is assayed in a leaf extract assay the results show an almost three fold stimulation of pYUCCA8:LUC activity (Figure 4B). Therefore, there is a discrepancy between the pYUCCA8:LUC activity detected in intact leaves and in leaf extracts. For this reason, we used only extract assay for the evaluation of the transient expression assays. One possible explanation is that untagged PIF4 activates many ATP consuming activities, resulting in a bigger competition for ATP and consequently a lower apparent LUC activity in living cells. In a leaf extract assay the ATP is provided and the cell physiology does not affect the quantification of LUC activity. However, at this point this explanation remains speculative. 
A)

In planta assay

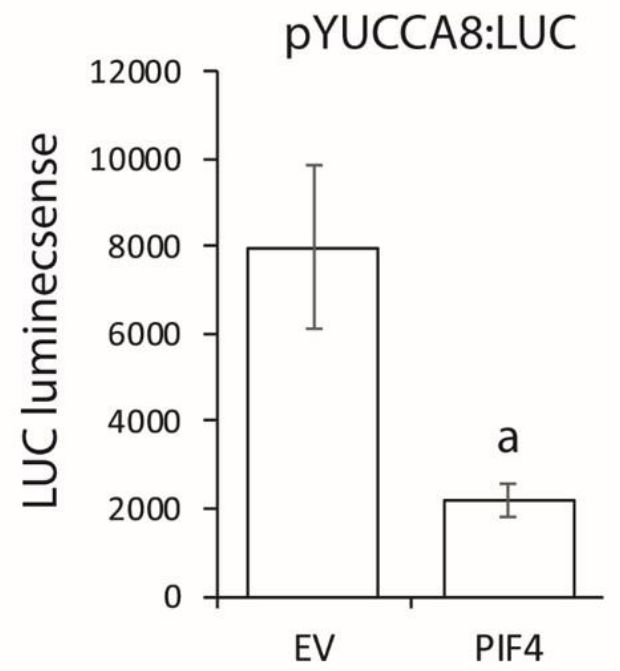

B) In vitro extract assay

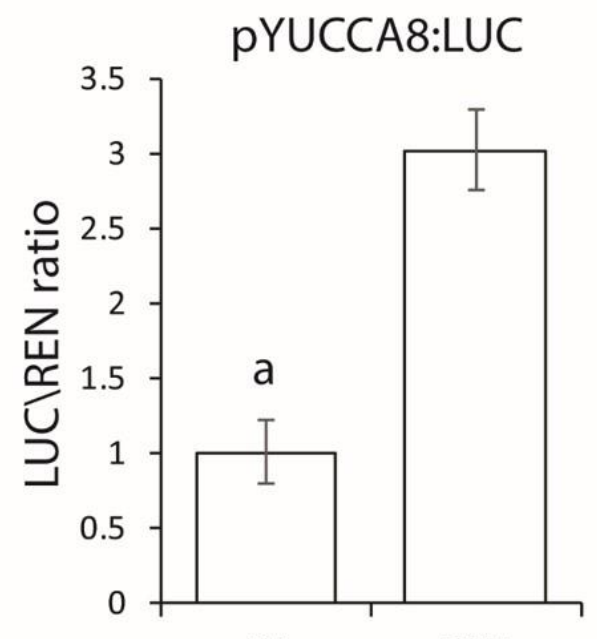

EV

PIF4

Figure 5. Difference in in planta LUC activity and LUC activity in leaf extacts. (A). Leaves of N.benthamiana were agro-infiltrated with the pYUCCA8-LUC reporter in combination with an empty vector construct or a 35S-PIF4 effector construct. LUC activity was imaged in leaves at 5 days post agroinfiltration. The relative LUC activity is quantified in ImageJ and adjusted for background signal. Number of replicate leaf: $\mathrm{N}=5$. Error bars represent mean $\pm S E$. (B) same agro infiltration experiment as in A but LUC activiaty was scored in leaf extracts instead of by imaging. The LUC activity is normalised by Renilla LUC from a 35S-renillaLUC expression construct used in each infiltration as control for agro-infiltration efficiency. Each average values is based on five biological and 3 technical replicates. Technical replicates were all similar ( $\Delta>1 \%)$. Significant differences between EV and PIF4 effector treatment are indicated by "a" ( $p$-value<0.05).

\section{PIF4 overexpression affects seedling hypocotyl elongation?}

In one of the first papers on PIF4, two lines are presented with overexpression of PIF4 without C-terminal extension (35S:PIF4). One line shows 1.1-fold and the other a 1.3-fold longer hypocotyl elongation for seedlings grown under R light (Huq and Quail, 2002). Our own transformation of Arabidopsis Col-0 with a 35S:PIF4 expression construct resulted in over 24 individual primary transformants. From these only 2 showed a substantial increase of hypocotyl elongation when grown under mixed light (Figure 5). This indicates that stimulation of hypocotyl elongation by PIF4OE under this condition is more the exception than the rule, or could vary depending on the growth conditions. However, when PIF4 with a C-terminal tag is overexpressed, the stimulation of hypocotyl elongation under $\mathrm{R}, \mathrm{WL}$ or $\mathrm{B}$ seems to be much stronger: 2,7-fold for PIF4-HA (Sun et al., 2012), 3-fold for PIF4-YFP and 2.5-fold for PIF4-myc (Ma et al., 2016), 4-fold for PIF4 GFP (de Lucas et al., 2008; Bernardo-Garcia et al., 2014). Therefore, we conclude that PIF4 proteins with tag can act as transcription factor, but that 
PIF4 with tag is more active in stimulating seedling hypocotyl elongation than PIF4 without Cor N-terminal tag. When the goal of an experiment is to assess whether PIF4 can stimulate gene expression for elongation, the PIF4+tag can still be used. However, when the goal of the experiment is to understand the regulation of PIF4 expression and regulation of PIF4 target genes, the use of PIF4+tag can give misleading results.

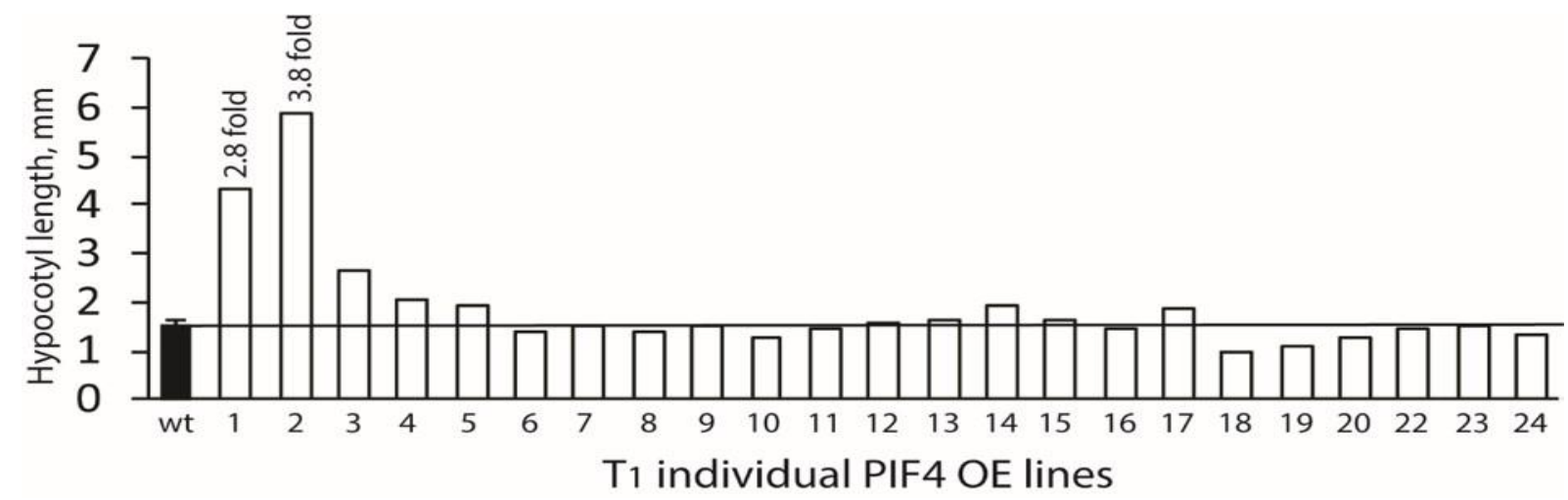

Figure 5. Distribution of the hypocotyl length in T1 PIF4OE lines. A total of 24 transgenic TO progeny seeds were selected by red seed coat marker. Seeds were imbibed on the MS-agar plate at $4{ }^{\circ} \mathrm{C}$ for four days. Then plates transferred to $12 \mathrm{hrWL} / 22^{\circ} \mathrm{C}$ and $12 \mathrm{hrD} / 18^{\circ} \mathrm{C}$. After 7 days the hypocotyl length of each $\mathrm{T} 1$ seedling was measured and compared to average length of WT hypocotyl $(N=17)$. The graph shows that only 2 PIF4OE lines have hypocotyl length more than 2-fold of WT hypocotyl length.

\section{PIF4 overexpression in MED25 does not link to hypocotyl elongation}

Another confusion result we obtained was during the analysis of the role of MED25 in elongation responses. The hypocotyl length of the MED25 mutant pft1-2 is not much affected under normal 12L/12D conditions compared to WT. However in pft1-2 seedlings the expression of PIF4 is substantially increased. Moreover, under warmth, the expression of PIF4 is stimulated in WT seedlings, resulting in increased hypocotyl elongation, while under warmth the expression of PIF4 is much stronger activated in pft1-2, but hypocotyl elongation is reduced compared to WT. Eventually we could show that this uncoupling of PIF4 activity and elongation responses may be linked to the uncoupling of PIF4 expression and PIF4 target gene expression in pft1-2. What made things also confusing was the expression of increased auxin signaling in pft1-2. To monitor auxin signalling in plants the artificial auxin sensing promoter eDR5v2 (Liao et al., 2015) was fused to the ffLUC coding region and the eDR5v2:LUC was introduced into WT plants. One of the representative eDR5V2:LUC reporter plants was 
developed into a homozygous reporter line. This line was crossed with pft1-2 to develop a pft1-2 ${ }^{\text {eDR5:LUC }}$ reporter line. Analysis of the auxin signalling activity in these plants show that eDR5v2:LUC activity is higher in pft1-2 (Figure 6), even though auxin biosynthetic gene activity YUCCA8 expression is lower in pft1-2: Chapter 5). We ascribe this higher auxin signalling activity to the role of MED25 in regulating transcriptional activity at promoters regulated by ARFs, as the eDR5v2 promoter is regulated by ARFs (Liao et al., 2015). When auxin levels are low, ARF activity is inhibited by Aux/IAA proteins and recently it was shown that this involves recruitment of a repressor complex that may also involve MED25 (Ito et al., 2016). At high auxin this repressor complex is released because of destruction of the AUX/IAA protein. Eventually the transcriptional activity of auxin-induced genes is counteracted by new production of AUX/IAA. We speculate that MED25 may play a role in recruitment of AUX/IAA and/or the repressor complex that binds to AUX/IAA proteins at auxin-induced genes after initial induction by auxin. MED25 is bound to the ARF7/19 at ARF target promoters and the mediator complex can interact with the dissociable CDK8 kinase module (CKM), which putatively blocks RNA polymerase II recruitment to targets of ARF7 and ARF19 (Ito et al., 2016). Part of the CKM complex is HEN3 (Wang and Chen, 2004) while HEN3 is bound to the transcription corepressor LEUNIG and the histone deacetylase HDA19 (Gonzalez et al., 2007). It is not known whether MED25 plays a direct role in recruiting this complex to ARF target promoters through interaction with HDA19. However, if HDA19, as part of the overall repressor complex, is recruited by specific interaction with MED25 to ARF target promoters, we predict that in a pft1-2 mutant background the auxin induced ARF transcriptional activity may be sustained longer when re-recruitment of the CKM repressive complex through interaction with HDA19 is less efficient without MED25. In the absence of MED25 auxininduced genes can apparently still be repressed, but the dynamics of suppression may be slower in the absence of MED25. The default higher eDR5V2:LUC activity as observed in pft12 may therefore be the result of prolonged auxin induced activity rather than an increased auxin induced activity. Indeed, when leaf tissues of WT TDR5:LUC $^{\text {or }} p f t 1-2^{\text {eDR5:LUC }}$ reporter plants are treated with auxin, the induced LUC activity in WT declines more rapid than in pft1-2 (Figure 6). This experiment was repeated four times with qualitatively similar results. However, the more prolonged auxin signalling activity in the pft1-2 mutant apparently does 
not cause increased elongation in contrast with the shorter hypocotyl or smaller rosette size of pft1-2 at $22^{\circ} \mathrm{C}$ (Chapter 4 . Figure 1 ).

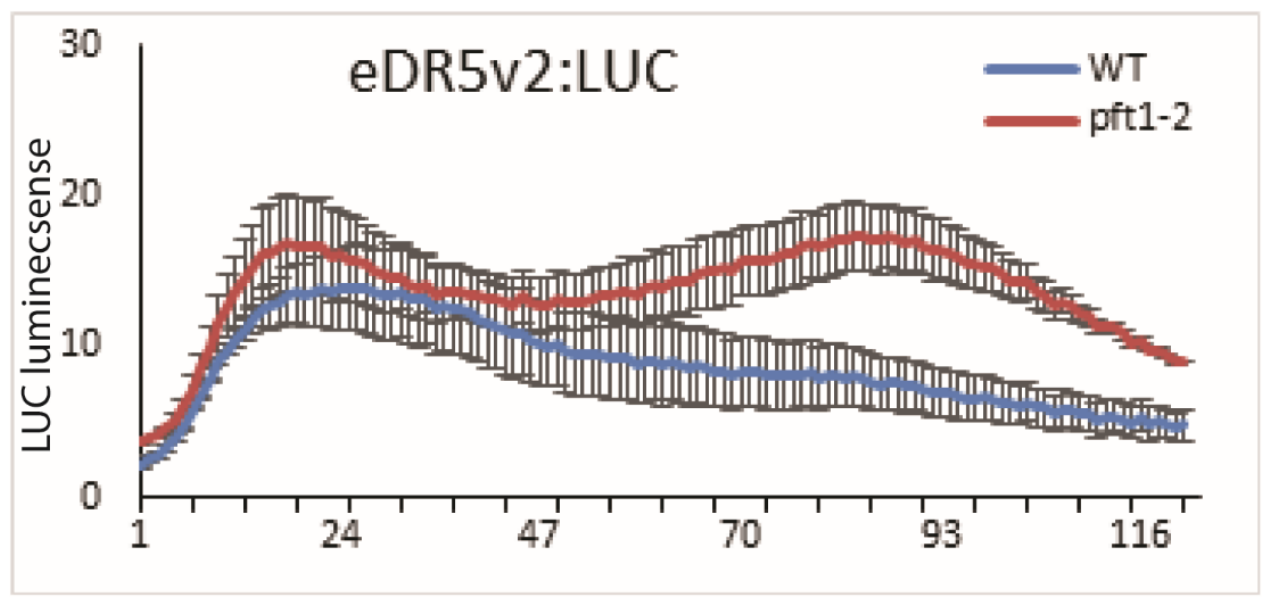

Figure 6. eDR5-LUC activity in response to auxin treatment. Leaf pieces of WT and pft1-2 expressing eDR5LUC were placed in $1 \mathrm{mM}$ luciferin and after 2 hours NAA was added to final concentration of $10 \mu \mathrm{M}$ NAA. Subsequently, the eDR5:LUC activity was imaged in continuous darkness every $10 \mathrm{~min}$. $\mathrm{Y}$ axis= relative LUC activity. $X$ axis= image numbers.

The uncoupling of PIF4 expression and elongation response in pft1-2 at normal temperature and during thermo-morphogenesis may be explained by the pleiotropic action of MED25 as MED25 associates with transcription factors involved in JA signalling (Kidd et al., 2009; Cevik et al., 2012; An et al., 2017), in auxin signalling (Raya-Gonzalez et al., 2014), in ABA signalling (Chen et al., 2012). For uncoupling of increased PIF4 expression or increased auxin signaling in pft1-2 and elongation, we predict a downstream component of the signal transduction pathway towards elongation (Figure 2) is affected in the pft1-2 mutant. For instance, such downstream target could be PIF3 (Bours et al., 2015). This will need further investigation in the future.

\section{-DIF elongation responses involve regulation of miRNAs}

As part of the "Compact plant" project, we performed RNA-seq on Arabidopsis plants under + DIF and -DIF for time point end-of-night (EON) and end-of-day (EOD) (M van Hoogdalem). For the same EOD and EON time points also microRNAs were isolated, with the aim to determine whether some of the differential mRNAs can be related to differential miRNA 
expression. MicroRNAs (miRNAs) are non-coding 21-24 nt small RNAs that function as post transcriptional regulators of gene expression in eukaryotes (Bartel, 2004). The mature miRNA may targets specific mRNA targets for degradation or for blocking mRNA translation. Since the first identification of a plant miRNA in 2002 (Reinhart et al., 2002), more than 1000 plant miRNAs have been identified (Zhang et al., 2005). Moreover, at present about 24500 miRNAs are registered in the Central Registry Database of MicroRNAs (www.mirbase.org, Release 20.0).

The -DIF response of plants is in part mediated through altered auxin responses and literature indicates that some of the genes that some of the auxin response genes are regulated by miRNAs. For instance, ath-miR167 and miR-160 suppress auxin signaling via cleavage of ARF6, ARF8 and ARF10 respectively (Wu et al., 2006; Liu et al., 2007). The overexpression of ath-miR319a results in small of plants and margined leaf shape (Shapulatov et al., 2018) and ath-miR156 target SPL genes which are involved in virtually every aspect of plant growth (Xie et al., 2017). This thesis has been about the role of PIFs in the DIF responses and PIF activity link to miRNAs by the fact that they can regulate expression of miRNA genes. For instance, it has been shown that PIF5 binds to promoter of MIR156 genes and repress MIR156 expression (Xie et al., 2017).

The analysis of the miRNAs isolated from the +DIF and -DIF experiment is still preliminary at this stage. All counts from miRNA samples per time point were combined and expression differences could only be determined from absolute counts and could not be based on statistical differences between treatments or time points. This limited results to only 4 miRNAs that showed at least a two-fold difference between +DIF and -DIF samples at EOD and EON. All these miRNAs are up regulated at EOD of -DIF and down-regulated at EON of DIF compared to the +DIF control (Figure 7). One of these miRNAs is ath-miR156d which targets SPL genes. Interestingly, it previously was shown that expression of the MIR156 genes is suppressed by PIFs (Xie et al., 2017). This result is in accordance with the effect of -DIF on PIF expression as determined by the RNA seq results, which show that under-DIF expression of PIF4 and PIF5 is significantly lower at EOD and significantly higher at EON (van Hoogdalem et al, unpublished). This indicates that part of the -DIF response on PIFs is translated into differential regulation of MIR156 miRNAs and downstream targets of miR156. It also shows 
that stimulation of elongation responses induced by shade or inhibition of elongation responses as under-DIF, are obtained through opposite regulation of the same set of components.

\section{Up}

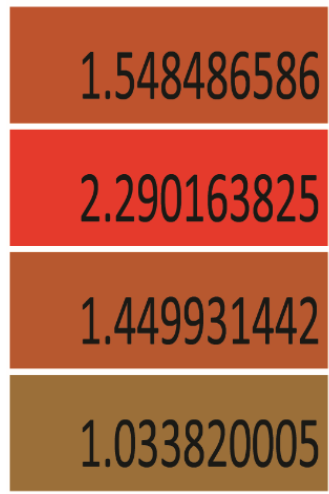

End of Day

-DIF

\section{Down}

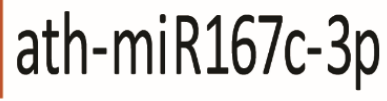

ath-miR396a-5p -1

ath-miR156d-3p

ath-miR396b-5p

End of Night -DIF

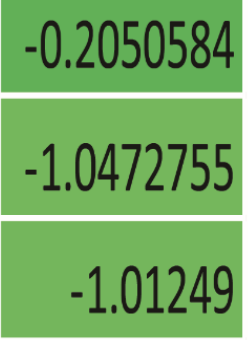

$-0.3254716$

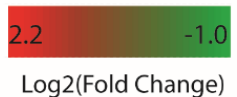

Figure-7. Profiling microRNA expression under negative DIF condition in Arabidopsis. A number of microRNAs from end of day (EOD) and end of night (EON) where up- or down-regulated under negative DIF condition. Heat map profile of four relevant microRNAs illustrates increased expression (red) and reduced expression (green) in DIF.

\section{Distractions: increased floral dip transformation frequency in pft1-2 and bzr1-1D}

The floral dip method is a widely-used technique to transform Arabidopsis by Agrobacterium tumefaciens (Zhang et al., 2006). The floral dip method is an in planta technique and does not require in vitro plant tissue culture or regeneration. Young floral buds are dipped into a suspension of Agrobacterium after which the Agrobacterium can penetrate the buds and reach the female gamete. Agrobacterium can than insert the T-DNA into the DNA of the female gamete and transformants can subsequently be selected from the seeds that have developed on the floral-dipped inflorescence (Zhang et al., 2006). Usually, the frequency at which transgenic seeds are found is between 0.1\%-1.0\% (Chung et al., 2000; Weigel and Glazebrook, 2006). I used floral dip to transform the pft1-2 and bzr1-1D mutants using a vector which contains the red-seed coat marker (Ali et al., 2012), making it easy to identify transformed seeds in the TO progeny after floral dip. During these experiments a higher 
transformation frequency was observed for the pft1-2 and bzr1-1D mutants and especially in the pft1-2/bzr1-1D double mutant compared to WT: per 100 ug seeds, 29 red seeds in WT, 61 in pft1-2, 70 in bzr1-1D and 110 in pft1-2/bzr1-1D. There can be several explanations for this difference in transformation frequency in the different mutant lines:

1) The transformation frequency could be related to the flower size of WT, single mutant and double mutant. It has been noted before that the pft1-2 mutant has larger floral organs compared to WT ( $\mathrm{P}=0.001$ ) (Xu and $\mathrm{Li}, 2011)$. We noted a similar larger floral organs in the gain of function mutant bzr1-1D (P=0.002) and in the pft1-2/bzr1-1D double mutant this effect on floral organ size is further enhanced (compared to bzr1-1D $\mathrm{P}=0.027$ ), indicating that BZR1 and MED25 have independent effects on floral organ size (Figure 8). The flower size does is affecting stigma size (not quantified) but does not noticeably affect the seed size. The increased flower size could result in more Agrobacterium penetrating the flower, or the difference in flower development could create a longer time window during which agrobacterium can transform ovules.

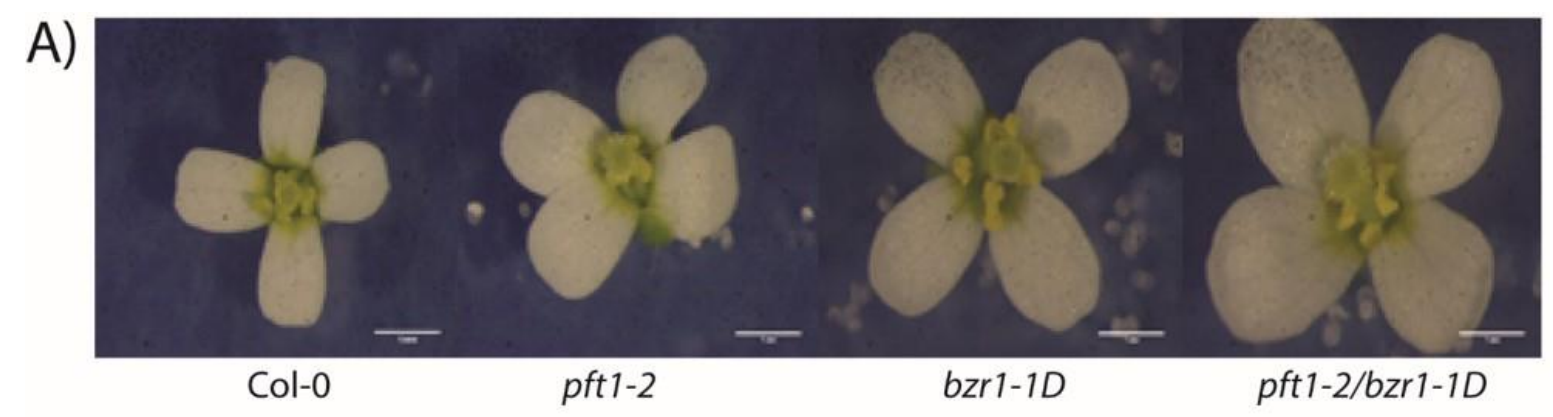

B)

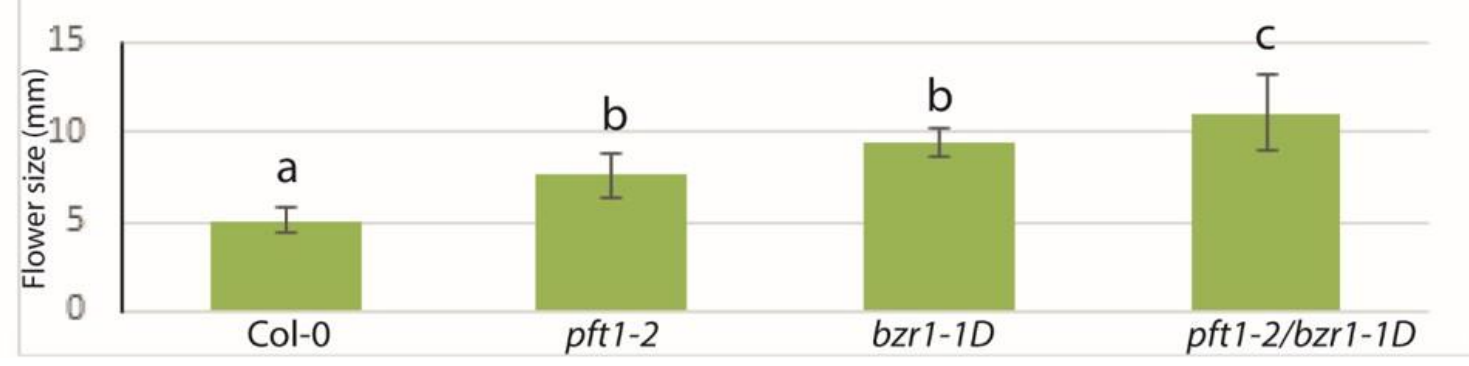

Figure 8. Flower size in WT, pft1-2, bzr1-1D and pft1-2/bzr1-1D. A) left to right: Representative flower of WT, pft1-2, bzr1-1D and pft1-2/bzr1-1D (scale bar = 1mm). B) Average flower areas $\left(\mathrm{mm}^{2}\right)$ per line including standard error bars $(n=8)$. Different letters indicate statistical differences at $P<0.05$. 
2) Alternatively, the difference in transformation frequency could be related to effects at the chromatin level in pft1-2 and bzr1-1D mutants. A number of bacterium proteins including VirD and VirE (Stachel and Nester, 1986; Eckardt, 2004) and in plants including Histone proteins (Lacroix et al., 2008) are participated during the T-DNA integration into host plant genome. Loss of function of $\mathrm{H} 2 \mathrm{~A}-1$ gene which encode core histone protein results in reduced T-DNA integration, while overexpression of this gene increased transformation frequency (Tenea et al., 2009). It has been shown before that mutants in histone modification show an altered TDNA transformation frequency (Gelvin and Kim, 2007). This would be consistent with the observations that MED25 interacts with HAC1 (An et al., 2017), HDA9 (Chapter 5).

3) The higher transformation could also be related to a lower resistance against Agrobacterium in pft1-2 due to lower SA levels. In the pft1-2 mutant and the bzr1-1D mutants the Salicylic Acid (SA) defence genes are less activated (Miyaji et al., 2014). Indeed it has been shown that SA negatively influences the growth of Agrobacterium (Anand et al., 2008).

Being able to manipulate the transformation frequency is of potential interest for transformation of recalcitrant crops. We therefore tried to confirm the results of different transformation frequencies in WT and the MED25 and BZR1 mutant lines. In this second experiment, the transformation frequencies in pft1-2 and bzr1-1D mutant background were again higher than in WT, but the double mutant did not show the highest transformation frequency. We did note that more of the different floral branches on pft1-2 and bzr1-1D contain siliques with transformed seeds (data not shown), suggesting that Agrobacterium may have a longer time window to do transformations in these mutants. This would support the $3^{\text {rd }}$ hypothesis of reduced defence against agrobacterium in these mutants, but additional research would be necessary to further address this.

\section{Future perspectives: research and applications}

This thesis is finished, but the work on understanding regulation of Phytochromes, PIF4, the role of MED25 and the translation of the activities of these components into elongation responses in plants is far from finished. While all studies here were done in Arabidopsis, the novel insights should be applicable to crops as well. PIFs have been studied in tomato, apple, 
grape and rice (Pham et al., 2017). Indications are that the basic interactions of PHYs with PIFs are conserved and that PIFs play similar roles in regulating elongation in crops. The number of interactions that I was able to uncover using the model system Arabidopsis would not have been possible when this research would have been done in a model crop. However, with the obtained knowledge, these studies can now be performed much faster in crops for validation and potential applications. Our findings of the light sensitivity of phytochrome gene expression especially under the artificial LED light conditions provide crucial new fundamental insights that may be used to control plant growth, yield and quality in greenhouses and indoor farming industries 


\section{Reference}

Ali MA, Shah KH, Bohlmann H (2012) pMAA-Red: a new pPZP-derived vector for fast visual screening of transgenic Arabidopsis plants at the seed stage. BMC Biotechnology 12: 37

An C, Li L, Zhai Q, You Y, Deng L, Wu F, Chen R, Jiang H, Wang H, Chen Q, Li C (2017) Mediator subunit MED25 links the jasmonate receptor to transcriptionally active chromatin. Proceedings of the National Academy of Sciences

Anand A, Uppalapati SR, Ryu CM, Allen SN, Kang L, Tang Y, Mysore KS (2008) Salicylic acid and systemic acquired resistance play a role in attenuating crown gall disease caused by Agrobacterium tumefaciens. Plant Physiol 146: 703-715

Bartel DP (2004) MicroRNAs: genomics, biogenesis, mechanism, and function. Cell 116: 281297

Bernardo-Garcia S, de Lucas M, Martinez C, Espinosa-Ruiz A, Daviere JM, Prat S (2014) BRdependent phosphorylation modulates PIF4 transcriptional activity and shapes diurnal hypocotyl growth. Genes Dev 28: 1681-1694

Bours R, Kohlen W, Bouwmeester HJ, van der Krol A (2015) Thermoperiodic control of hypocotyl elongation depends on auxin-induced ethylene signaling that controls downstream PHYTOCHROME INTERACTING FACTOR3 activity. Plant Physiol 167: 517530

Bours R, van Zanten M, Pierik R, Bouwmeester H, van der Krol A (2013) Antiphase light and temperature cycles affect PHYTOCHROME B-controlled ethylene sensitivity and biosynthesis, limiting leaf movement and growth of Arabidopsis. Plant Physiol 163: 882-895

Carvalho SMP, Heuvelink E, Cascais R, Van Kooten O (2002) Effect of Day and Night Temperature on Internode and Stem Length in Chrysanthemum: Is Everything Explained by DIF? Annals of Botany 90: 111-118

Cevik V, Kidd BN, Zhang P, Hill C, Kiddle S, Denby KJ, Holub EB, Cahill DM, Manners JM, Schenk PM, Beynon J, Kazan K (2012) MEDIATOR25 acts as an integrative hub for the regulation of jasmonate-responsive gene expression in Arabidopsis. Plant Physiol 160: 541-555

Chen R, Jiang H, Li L, Zhai Q, Qi L, Zhou W, Liu X, Li H, Zheng W, Sun J, Li C (2012) The Arabidopsis mediator subunit MED25 differentially regulates jasmonate and abscisic acid signaling through interacting with the MYC2 and ABI5 transcription factors. Plant Cell 24: 2898-2916

Chen X, Lu L, Mayer KS, Scalf M, Qian S, Lomax A, Smith LM, Zhong X (2016) POWERDRESS interacts with HISTONE DEACETYLASE 9 to promote aging in Arabidopsis. Elife $\mathbf{5}$

Choi H, Oh E (2016) PIF4 Integrates Multiple Environmental and Hormonal Signals for Plant Growth Regulation in Arabidopsis. Molecules and Cells 39: 587-593

Chung M-H, Chen M-K, Pan S-M (2000) Floral Spray Transformation Can Efficiently Generate Arabidopsis. Transgenic Research 9: 471-486

de Lucas $M$, Daviere JM, Rodriguez-Falcon M, Pontin M, Iglesias-Pedraz JM, Lorrain S, Fankhauser C, Blazquez MA, Titarenko E, Prat S (2008) A molecular framework for light and gibberellin control of cell elongation. Nature 451: 480-484

Eckardt NA (2004) Host Proteins Guide Agrobacterium-Mediated Plant Transformation. The Plant Cell 16: 2837 
Franklin KA, Lee SH, Patel D, Kumar SV, Spartz AK, Gu C, Ye S, Yu P, Breen G, Cohen JD, Wigge PA, Gray WM (2011) Phytochrome-interacting factor 4 (PIF4) regulates auxin biosynthesis at high temperature. Proc Natl Acad Sci U S A 108: 20231-20235

Gangappa SN, Berriri S, Kumar SV (2017) PIF4 Coordinates Thermosensory Growth and Immunity in Arabidopsis. Curr Biol 27: 243-249

Gelvin SB, Kim SI (2007) Effect of chromatin upon Agrobacterium T-DNA integration and transgene expression. Biochim Biophys Acta 1769: 410-421

Gonzalez D, Bowen AJ, Carroll TS, Conlan RS (2007) The transcription corepressor LEUNIG interacts with the histone deacetylase HDA19 and mediator components MED14 (SWP) and CDK8 (HEN3) to repress transcription. Mol Cell Biol 27: 5306-5315

Hornitschek P, Kohnen MV, Lorrain S, Rougemont J, Ljung K, Lopez-Vidriero I, Franco-Zorrilla JM, Solano R, Trevisan M, Pradervand S, Xenarios I, Fankhauser C (2012) Phytochrome interacting factors 4 and 5 control seedling growth in changing light conditions by directly controlling auxin signaling. Plant J 71: 699-711

Hornitschek P, Lorrain S, Zoete V, Michielin O, Fankhauser C (2009) Inhibition of the shade avoidance response by formation of non-DNA binding bHLH heterodimers. EMBO J 28: 3893-3902

Huq E, Quail PH (2002) PIF4, a phytochrome-interacting bHLH factor, functions as a negative regulator of phytochrome $B$ signaling in Arabidopsis. EMBO J 21: 2441-2450

Ibañez C, Delker C, Martinez C, Bürstenbinder K, Janitza P, Lippmann R, Ludwig W, Sun H, James GV, Klecker M, Grossjohann A, Schneeberger K, Prat S, Quint M (2018) Brassinosteroids Dominate Hormonal Regulation of Plant Thermomorphogenesis via BZR1. Current Biology 28: 303-310.e303

Ito J, Fukaki H, Onoda M, Li L, Li C, Tasaka M, Furutani M (2016) Auxin-dependent compositional change in Mediator in ARF7- and ARF19-mediated transcription. Proc Natl Acad Sci U S A 113: 6562-6567

Jung JH, Domijan M, Klose C, Biswas S, Ezer D, Gao M, Khattak AK, Box MS, Charoensawan V, Cortijo S, Kumar M, Grant A, Locke JC, Schafer E, Jaeger KE, Wigge PA (2016) Phytochromes function as thermosensors in Arabidopsis. Science 354: 886-889

Kidd BN, Edgar Cl, Kumar KK, Aitken EA, Schenk PM, Manners JM, Kazan K (2009) The mediator complex subunit PFT1 is a key regulator of jasmonate-dependent defense in Arabidopsis. Plant Cell 21: 2237-2252

Koini MA, Alvey L, Allen T, Tilley CA, Harberd NP, Whitelam GC, Franklin KA (2009) High temperature-mediated adaptations in plant architecture require the bHLH transcription factor PIF4. Curr Biol 19: 408-413

Kunihiro A, Yamashino T, Nakamichi N, Niwa Y, Nakanishi H, Mizuno T (2011) Phytochromeinteracting factor 4 and 5 (PIF4 and PIF5) activate the homeobox ATHB2 and auxininducible IAA29 genes in the coincidence mechanism underlying photoperiodic control of plant growth of Arabidopsis thaliana. Plant Cell Physiol 52: 1315-1329

Lacroix B, Loyter A, Citovsky V (2008) Association of the \&lt;em\&gt;Agrobacterium\&lt;/em\&gt; T-DNA-protein complex with plant nucleosomes. Proceedings of the National Academy of Sciences 105: 15429

Legris M, Klose C, Burgie ES, Rojas CC, Neme M, Hiltbrunner A, Wigge PA, Schafer E, Vierstra RD, Casal JJ (2016) Phytochrome B integrates light and temperature signals in Arabidopsis. Science 354: 897-900 
Li J, Li G, Wang H, Wang Deng X (2011) Phytochrome Signaling Mechanisms. The Arabidopsis Book / American Society of Plant Biologists 9: e0148

Liao CY, Smet W, Brunoud G, Yoshida S, Vernoux T, Weijers D (2015) Reporters for sensitive and quantitative measurement of auxin response. Nat Methods 12: 207-210, $202 p$ following 210

Liu PP, Montgomery TA, Fahlgren N, Kasschau KD, Nonogaki H, Carrington JC (2007) Repression of AUXIN RESPONSE FACTOR10 by microRNA160 is critical for seed germination and post-germination stages. Plant J 52: 133-146

Ma D, Li X, Guo Y, Chu J, Fang S, Yan C, Noel JP, Liu H (2016) Cryptochrome 1 interacts with PIF4 to regulate high temperature-mediated hypocotyl elongation in response to blue light. Proc Natl Acad Sci U S A 113: 224-229

Martínez C, Espinosa-Ruíz A, de Lucas M, Bernardo-García S, Franco-Zorrilla JM, Prat S (2018) PIF4-induced BR synthesis is critical to diurnal and thermomorphogenic growth. The EMBO Journal

Miyaji T, Yamagami A, Kume N, Sakuta M, Osada H, Asami T, Arimoto Y, Nakano T (2014) Brassinosteroid-related transcription factor BIL1/BZR1 increases plant resistance to insect feeding. Biosci Biotechnol Biochem 78: 960-968

Nomoto Y, Kubozono S, Miyachi M, Yamashino T, Nakamichi N, Mizuno T (2012) A circadian clock- and PIF4-mediated double coincidence mechanism is implicated in the thermosensitive photoperiodic control of plant architectures in Arabidopsis thaliana. Plant Cell Physiol 53: 1965-1973

Oh E, Zhu JY, Wang ZY (2012) Interaction between BZR1 and PIF4 integrates brassinosteroid and environmental responses. Nat Cell Biol 14: 802-809

Pham VN, Kathare PK, Huq E (2017) Phytochromes and Phytochrome Interacting Factors. Plant Physiology

Quint M, Delker C, Franklin KA, Wigge PA, Halliday KJ, van Zanten M (2016) Molecular and genetic control of plant thermomorphogenesis. Nat Plants 2: 15190

Raya-Gonzalez J, Ortiz-Castro R, Ruiz-Herrera LF, Kazan K, Lopez-Bucio J (2014) PHYTOCHROME AND FLOWERING TIME1/MEDIATOR25 Regulates Lateral Root Formation via Auxin Signaling in Arabidopsis. Plant Physiol 165: 880-894

Reinhart BJ, Weinstein EG, Rhoades MW, Bartel B, Bartel DP (2002) MicroRNAs in plants. Genes Dev 16: 1616-1626

Shapulatov U, van Hoogdalem M, Schreuder M, Bouwmeester H, Abdurakhmonov IY, van der Krol AR (2018) Functional intron-derived miRNAs and host-gene expression in plants. Plant Methods 14: 83

Stachel SE, Nester EW (1986) The genetic and transcriptional organization of the vir region of the A6 Ti plasmid of Agrobacterium tumefaciens. The EMBO journal 5: 1445-1454

Stavang JA, Lindgård B, Erntsen A, Lid SE, Moe R, Olsen JE (2005) Thermoperiodic stem elongation involves transcriptional regulation of gibberellin deactivation in pea. Plant physiology 138: 2344-2353

Sun J, Qi L, Li Y, Chu J, Li C (2012) PIF4-mediated activation of YUCCA8 expression integrates temperature into the auxin pathway in regulating arabidopsis hypocotyl growth. PLoS Genet 8: e1002594

Sun J, Qi L, Li Y, Chu J, Li C (2012) PIF4-Mediated Activation of YUCCA8 Expression Integrates Temperature into the Auxin Pathway in Regulating Arabidopsis Hypocotyl Growth. PLOS Genetics 8: e1002594 
Sun J, Qi L, Li Y, Zhai Q, Li C (2013) PIF4 and PIF5 transcription factors link blue light and auxin to regulate the phototropic response in Arabidopsis. Plant Cell 25: 2102-2114

Tasset C, Singh Yadav A, Sureshkumar S, Singh R, van der Woude L, Nekrasov M, Tremethick D, van Zanten M, Balasubramanian S (2018) POWERDRESS-mediated histone deacetylation is essential for thermomorphogenesis in Arabidopsis thaliana. PLOS Genetics 14: e1007280

Tenea GN, Spantzel J, Lee L-Y, Zhu Y, Lin K, Johnson SJ, Gelvin SB (2009) Overexpression of Several \&lt;em\&gt;Arabidopsis\&lt;/em\&gt; Histone Genes Increases \&lt;em\&gt;Agrobacterium\&lt;/em\&gt;-Mediated Transformation and Transgene Expression in Plants. The Plant Cell 21: 3350

Wang W, Chen X (2004) HUA ENHANCER3 reveals a role for a cyclin-dependent protein kinase in the specification of floral organ identity in Arabidopsis. Development 131: 31473156

Weigel D, Glazebrook J (2006) In planta transformation of Arabidopsis. CSH Protoc 2006

Wu J, Wang W, Xu P, Pan J, Zhang T, Li Y, Li G, Yang H, Lian H (2018) phyB Interacts with BES1 to Regulate Brassinosteroid Signaling in Arabidopsis. Plant and Cell Physiology: pcy212pcy212

Wu MF, Tian Q, Reed JW (2006) Arabidopsis microRNA167 controls patterns of ARF6 and ARF8 expression, and regulates both female and male reproduction. Development 133: 4211-4218

Xie Y, Liu Y, Wang H, Ma X, Wang B, Wu G, Wang H (2017) Phytochrome-interacting factors directly suppress MIR156 expression to enhance shade-avoidance syndrome in Arabidopsis. Nature Communications 8: 348

Xu R, Li Y (2011) Control of final organ size by Mediator complex subunit 25 in Arabidopsis thaliana. Development 138: 4545-4554

Zhang BH, Pan XP, Wang QL, Cobb GP, Anderson TA (2005) Identification and characterization of new plant microRNAs using EST analysis. Cell Res 15: 336-360

Zhang X, Henriques R, Lin SS, Niu QW, Chua NH (2006) Agrobacterium-mediated transformation of Arabidopsis thaliana using the floral dip method. Nat Protoc 1: 641646 


\section{Summary}

Light and temperature signalling response is a central mediator of plant growth plasticity. This thesis aimed to provides new molecular insights into control of plant growth. This may have its application in greenhouses for improved growth control under artificial light and temperature conditions. In the introduction chapter $\mathbf{1}$, I describe the history and context of research on light/temperature regulation of plant growth. I provide background information on how phytochromes play an important role in the response to plant elongation, especially as function of the R:FR ratio in the light spectrum. The input phytochrome protein level is determined by transcription of the different PHY genes and the role of transcriptional regulation of PHY genes has not been investigated extensively. Using PHY-LUC reporter plants it was shown that at the seedling stage PHY gene expression shows oscillations under continuous white light, indicating that PHY genes are under control of the circadian clock. In this thesis one of the major research questions was to determine the transcriptional regulation of $\mathrm{PHY}$ genes in Arabidopsis under different light conditions, to determine what the potential input in PHY protein levels is during the photoperiod at which PHYs are activated. Ultimately this can then also answer the question whether PHY protein levels can be limiting or saturating for PIF protein stability. In experimental chapter 2, I investigated the role of phytochrome gene activity as function of light quality and temperature. Five PHY-LUC reporter lines were constructed and each of the PHY-LUC reporters were also crossed in phytochrome single KO mutant backgrounds. Analysis of the LUC activity in seedlings and rosette plants were done under mixed LED (consisting of R, FR and B), pure R, pure Fr and pure B light. In this way we made several new discoveries: (1) At the seeding stage there are many interactions between the different PHY genes, as PHY-LUC activity was substantially affected in the different single PHY mutants. However, most of these interactions were gone in the mature rosette stage of Arabidopsis. (2) we found that PHYD is a consistent repressor of PHYA transcriptional activity. (3) we discovered strong and direct upregulation of PHYB under FR light, which is not dependent on PHYA (the usual regulator of FR responses), but was dependent in part on PHYE and in part on PHYB. (4) we observed a slow, but steady and ultimately strong increase in PHYA expression under FR, which is not dependent on PHYA, but on PHYE. (5) overall the results identify PHYE as a possible novel sensor for FR light. The 
consequences of these strong effects of FR LED light on PHYA and PHYB expression need further exploration and needs to be linked to transcription factors that are activated under FR light. Future research will have to show whether the strong manipulation of PHY expression by pure FR LED light can be mobilised for growth control in greenhouses.

The experimental chapter 3 is about the complex feedback regulation at the PIF4 locus. PIF4 functions as a hub in the control of plant growth and plant resilience and a full understanding of PIF4 gene regulation is therefore crucial for understanding plant growth and resilience. During this thesis work it became known that BZR1 is a key transcriptional regulator of PIF4 expression, especially under higher temperature. This regulation by BZR1 is part of an indirect positive feedback loop through BR synthesis, BR signaling and further activation of BZR1. Such positive feedback has the danger of unrestricted increase in PIF4 and BZR1 activity. However, it was thought that this positive feedback regulation of PIF4 is kept under control by factors acting at the post-transcriptional level. We discovered that this positive feedback regulation by BZR1 is actually broken by PIF4 itself, which acts as a negative regulator of its own expression. The negative action of PIF4 can also compete with the positive action of BZR1. Overall this adds a new layer to the regulation of transcription of PIF4 and shows that overall PIF4 transcription may be determined by the relative levels of PIF4 to BZR1 protein. Near the very end of this thesis work a publication revealed the action of PIF4 and BZR1 on genes of the BR biosynthesis pathway. For these BR biosynthetic genes BZR1 homodimers act as a repressor and heterodimer formation between PIF4 and BZR1 lead to a release of the BZR1 homodimer repressor from these target promoters. It was not clear whether excess PIF4 is subsequently acting as positive factor on these target promoters. We propose a similar model for the regulation of PIF4, but with the change of BZR1 homodimer acting as positive factor for PIF4 transcription. When PIF4 protein levels rise, formation of the PIF4/BZR1 heterodimer removes the positive acting BZR1 homodimer. It is not clear whether subsequently excess PIF4 than acts as negative factor for its own gene transcription. In addition, we show in chapter 3 that PIF4 regulate PHYB and PHYD expression. As light activated PHY protein leads to destruction of PIF4, this adds another indirect feedback on PIF4 activity.

In experimental chapter 4 we investigated the role of MED25 in transcriptional regulation of PIF4. MED25 is a plant specific component of the Mediator complex, which acts 
between transcription factors bound to promoters and the general transcription machinery containing Polymerase II. First we discovered that in the MED25 mutant pft1-2 the hypocotyl elongation under warmth is attenuated. Moreover, we demonstrate that MED25 can interact with PIF4 and BZR1, adding these two transcription factors to the subset of transcription factors that can interact with MED25 (unpublished data). We subsequently show that PIF4 expression is upregulated in pft1-2 at ambient temperature, while PIF4 expression is super induced compared to WT at warm temperature. However, this upregulation of PIF4 expression is uncoupled from induction of PIF4 target gene YUCCA8 and from the elongation response. The regulation of PIF4 gene transcription and regulation of PIF4 target genes by PIF4 has an additional layer, as histone modifications at these promoters also become part of the equation. We provide evidence that MED25 may actually recruit histone modifying activity for PIF4 target genes. During this thesis research it was shown that the SANT (SWI3/DAD2/NCoR/TFIII-B) domain protein POWERDRESS (PWR) acts as a subunit in a complex with HDA9 to result in lysine deacetylation of histone $\mathrm{H3}$ at specific genomic targets. We assisted M.van Zanten from Utrecht University in elucidating the role of HDA9 in transcriptional activity of PIF4 target genes under warmth by monitoring different LUC reporters at $22^{\circ} \mathrm{C}$ and $27^{\circ} \mathrm{C}$. These studies led to a model in which HDA9 is stabilized under warmth and is recruited to PIF4 target promoters to facilitate local histone deacetylaton. This in turn facilitates exchange of repressive $\mathrm{H} 2 \mathrm{~A} . \mathrm{Z}$ histones at these promoters for permissive canonical H2A histones, which are evicted more rapidly under warmth and thus freeing the promoter for a positive PIF4 action. Unclear in this model was how HDA9 is stabilized under warmth and how HDA9 is recruited to these specific promoter sites. This question was addressed by our studies which show that MED25 can bind both to PIF4, BZR1 and to HDA9. A role for MED25 in recruiting histone modifying activity has previously been described for Jasmonic Acid (Ja-lle) signaling responses, during which MED25, bound to MYC2 at MYC2 target promoters can recruit Histone acetylase enzyme (HAC1). However, in this context MED25 recruits a HAC1 which activates transcription of the MYC2 target genes. Mediator is also involved in recruiting HDA19 to promoters regulated by ARF7 and ARF19, while MED25 can bind to these ARFs. However, in the context of ARF regulated promoters it is not yet clear whether MED25 plays a direct role in recruitment of HDA19. 
Finally, in experimental chapter 5 I describe a novel strategy by which plants can be transformed with a single construct to obtain overexpression of the transgene and silencing of an endogenous target gene of interest. This strategy was inspired by observing that a few plant genes in nature contain a miRNA encoded in an intron. Although for plant microRNA containing genes it has not been fully investigated whether such genes can produce the two potential products of host gene encoded protein and intron encoded miRNA I used this concept to design a transgene with intron in which an artificial intron miRNA (aimiRNA) was placed. As host gene the sequence of the firefly luciferase with intron was used and as miRNA template we used the miRNA319a sequence, also because the activity of this miRNA gives a clear plant leaf phenotype. After adjusting the positioning of the aimiRNA within the intron we obtained several examples of a working transgene which also produces a functional miRNA. Such concept may now be combined with the research we did on plant growth control, for instance by making a bzr1-1D overexpression construct with a miRNA in the intron that targets MED25. Such single construct could be used to transform ornamentals to test whether larger flowers are formed for crops, like cotton to determine if this allows for more cotton fiber elongation.

In the final discussion chapter, I discuss some of the many questions that remain after this research. In general this research has been broad, leading to many new discoveries, but some discoveries now need follow up to get a full understanding of the underlying mechanism. 


\section{Acknowledgements}

After four years spent my PhD journey in Wageningen U\&R, now I would say it was most challenging and grateful time in my career. I would like to special thanks to Erasmus Mundus Action 2 projects TIMUR for three years scholarship and STW Compact plant project for funding the thesis research. To complete this work, many people were contributed as direct and indirect, but I would like to address some of you that played a pivotal role during this period.

Firstly, I would like to express my sincere gratitude to my dear supervisor Sander van der Krol for the daily support of my PhD study and passion for research. This thesis would not have been possible without your effort and help. You gave me such as opportunity where I feel more independent to work in Lab. Your door was always open whenever I had questions about the topic. Therefore, we had wonderful discussions to make new ideas where I never hesitated to test it. I still remember when you said "Don't ignore negative results; it gives a lot of information to go next step". Also, some of your critical points to our outputs were positively influenced to my scientific thinking. You taught me in all the time to successfully finish the experiment and understand what does mean the result. I feel very satisfied to receive enough support and guidance from your supervision. Besides my supervisor, the powerful thanks to several peoples who are directly involved Sander's project: Wim, Mark, Habtamu, Marielle, Elias, Jacqueline and Lidiya for teaching me the Lab methods, contribution to the experiments and critical comments to my work.

I would like to thanks to Christa Testerink for hands on supervision and correction of the manuscripts as a chapters in this thesis. Your supports helped me to feel as a part of Plant Physiology Lab. Thanks Harro Bouwmeester, you received me to join PPH Lab and helped all the formalities of WUR as a promoter for my two years of my PhD in WUR. Ibrokhim, thank you for your contribution and scientific comments to my research. I would also like to thanks to Ernst for encouraging me during the PhD study.

I would also like to express my warm thanks to the staff members of PPH, Henk, Wilco, Leonie, Dick, Iris, Richard, Lot, secretariats Rina, Margaret and technicians Marielle, Francel, Lidiya, Leo, Jessica for sharing your knowledge, effective supervision during the literature discussion, providing data bases and other relevant sources. Your advises helped me to making the right decision in Lab work.

Mark, you are my best friend in the Netherlands. We have worked together in same project as a PhD candidate. Together discussion on the topic, we have shared our knowledges and plant materials. You have been extremely supportive through some difficult time. I am very glad that we became a good friend which I will keep in tough for future as well. 
It is my pleasure to thanks Arman, Margriet, Mark L, Jacqueline, Rumyana, Bing, Yanxia, Jinbin, without your helps and supports my experiments would have not been done. Giving your scientific advices has motivated me to finish my work; your suggestions provided to make easy life in Wageningen.

It was much easy to join PPH student community because, friendly PhD candidates always surrounded me. I became very closer to you guys during the PPH PhD trip. Later, I found other colleagues and friends that I so much enjoyed to spend my time with you. For that I want to appreciate Sangseok, Gonda, Mara, Esmer, Emilie, Beatriz, Elise, Jun, Wei, Bo, Nafiseh, Natalia, Melissa, Mariana, Alexandre, Bas, Karen, Cecillia, Shuang, Yuanyuan, Renake, Farzaneh, Mahdere, Giovanni, Hal, Yanting, Caroline, Diaan, Krystina, Juriaan, Mohammed, my students (Yameng, Christa, Job, Laurens, Alexander) and other peoples.

I would also like to thanks to other new PhD candidates: Nikita, Ayodeji, Iko, Alice, Carmen, Jasper, Eva, Irini, Yutao and Hongfei where started recently in PPH group for sharing your supplements in Lab and warm communication in coffee corner, outside trip et cetera. Sometime I would say myself, I met many scientists and made good friendship. Hope we will keep good contact in the future.

I also want to thanks all Uzbek students, where we have studied in different departments and subjects in WUR. We organised a several scientific - cultural events and travelled together around Netherlands. We cocked some our national cuisines and celebrated Uzbek holidays in this city to feel as at home.

Ushbu muvaffaqiyatga erishishimda dadam va onamning bergan o'gitlari va talim-tarbiyasi beqiyos o'rin tutadi. Shuningdek, ilm-fanga bo'Igan qiziqishimni rag'batlantirgan barcha uztozlarimga o'z minnatdorchiligimni bildirib qolaman.

\section{With sincerely,}

\section{Hurmat bilan,}

\section{Umidjon Shapulatov}




\section{UMIDJON SHAPULATOV}

Date of Birth: Oct 6, 1984 in Sayxunobod district, Uzbekistan

Contact: umidjon.shapulatov@gmail.com

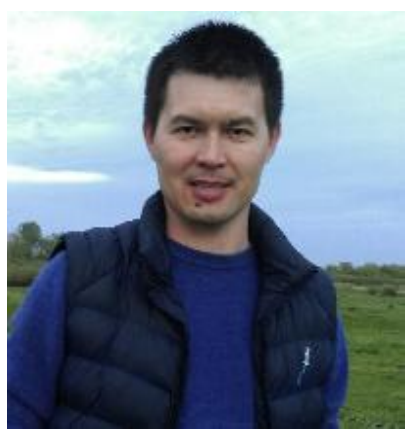

\section{Education and Research Experience:}

\section{Wageningen University, The Netherlands}

PhD candidate in the Laboratory of Plant Physiology

Dec 2014y- Dec 2018y

Supervisors: Prof. Christa Testerink and Dr. Sander van der Krol

Center of Genomics and Bioinformatics, Academy of Science of the Republic of Uzbekistan $2012 \mathrm{y}-2014 \mathrm{y}$

Researcher

Institute of Genetics and Plant Experimental Biology, Academy of Science of the Republic of Uzbekistan $2007 y-2012 y$

Researcher

\section{National University of Uzbekistan}

Master's degree, Laboratory of Biotechnology and Microbiology

Sep 2005 - June 2007

Supervisors: Prof. I.Y.Abdurakhmanov and Prof. Sh. Tashmukhamedova

Gulistan State University, Uzbekistan

Bachelor's degree, Faculty of Life Science

Sep 2001 - June 2005

Supervisors: Prof. Kh.Kushiev and Dr T. Kuliev

\section{Expertise and Research Interest}

Light signalling, shade avoidance, chromatin modification and transcriptional regulation, thermomorphogenesis in plants, microRNA silencing, Luciferase reporter system;

\section{Publications}

Umidjon Shapulatov, Mark van Hoogdalem, Marielle Schreuder, Harro Bouwmeester, Ibrokhim Y. Abdurakhmonov and Alexander R. van der Krol. Functional intron-derived miRNAs and host-gene expression in plants. Plant Methods (2018) 14:83;

U. M. Shapulatov, Z. T. Buriev, M. Ulloa, S. Saha, E. J. Devor, M. S. Ayubov,T. M. Norov, S. E. Shermatov, A. Abdukarimov, J. N. Jenkins, I. Y. Abdurakhmonov. Characterization of Small RNAs and Their Targets from Fusarium oxysporum Infected and Noninfected Cotton Root Tissues. Plant Mol Biol Rep (2016) 34:698-706; 
Education Statement of the Graduate School

Experimental Plant Sciences

Issued to: Umidjon Shapulatov

Date: $\quad 02$ April 2019

Group: Laboratory of Plant Physiology

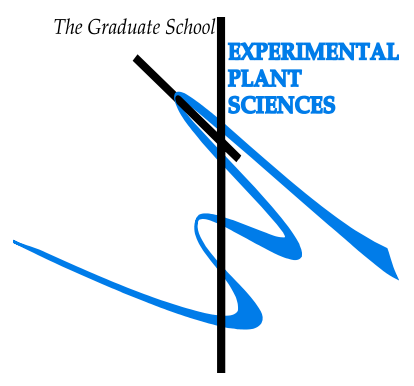

University: Wageningen University \& Research

1) Start-Up Phase

- First presentation of your project

Understanding environmental control of signal capacity towards plant growth responses

- Writing or rewriting a project proposal

Light/Temperature control of cell elongation as function of phytochromes, microRNAs and alternative splicing

- Writing a review or book chapter

- MSc courses

Subtotal Start-Up Phase

2) Scientific Exposure

- EPS PhD student days

Annual EPS PhD student day (Get2Gether 2017), Soest, The Netherlands

Annual EPS PhD student day (Get2Gether 2018), Soest, The Netherlands

- EPS theme symposia

EPS Theme 1 Symposium: Plant Development. Wageningen U\&R, The Netherlands

EPS Theme 1 Symposium: Plant Development. Leiden, The Netherlands

EPS Theme 1 Symposium: Plant Development. Wageningen U\&R, The Netherlands

EPS Theme 1 Symposium: Plant Development. Leiden, The Netherlands

National meetings (e.g. Lunteren days) and other National

- Platforms

Annual meeting "Experimental Plant Sciences", Lunteren, The Netherlands

Annual meeting "Experimental Plant Sciences", Lunteren, The Netherlands

Annual meeting "Experimental Plant Sciences", Lunteren, The Netherlands

Annual meeting "Experimental Plant Sciences", Lunteren, The Netherlands

- Seminars (series), workshops and symposia

Seminar: "And yet they oscillate: functional analysis of circadian long non-coding RNAs", Rossana Henriques

Seminar:"The response to cold stress in rice: signaling, transcriptional and metabolic regulation" Jingyu Zhang Symposium: Erasmus Mundus TIMUR General Assamble

09-10 Feb 2017

15-16 Feb 2018

0.6

21 Jan 2016

0.3

28 Feb 2017

0.3

30 Jan 2018

0.3

31 Jan 2019

0.3

13-14 Apr 2015

0.6

11-12 Apr 2016

0.6

10-11 Apr 2017

0.6

09-10 Apr 2018

0.6

16 Nov 2015

0.1

01 Dec 2015

0.1

14-15 Mar 2016 
Seminar:"Genomics-enabled natural products discovery", Douglas Mitchel

Seminar:"How Ralstonia solanacearum succeeds in plant xylem vessels", Caitilyn Allen

Seminar:"The evolution of branching mechanisms", Jill Harrison Seminar:"Signalling networks in plant responses to shade", Jorge Casal

Seminar:"Finding balance to optimize plant fitness", Stephan Pollmann

Seminar: The immune receptor $\mathrm{Rx} 1$ remodels chromatin and chromatin interactors in immunity", Martin Cann

Seminar:"Designing STRONG rice for dry and saline lands" Seminar: "Salicylic acid and cell cycle control of plant-microbe interactions", Mary C. Wildermuth

Symposium: 7th Dutch Seed Symposium, Wageningen, The Netherlands

\section{- Seminar plus}

\section{- International symposia and congresses}

Thermomorphogenesis conference, Halle, Germany

9th International Utrecht PhD Summer School on Environmental Signaling in Plants, Utrecht, NL

IGC Symposium "Plant RNA Biology", Oeiras, Portugal 10th European Plant Science Retreat (EPSR), Utrecht, NL

Thermomorphogenesis conference, Utrecht, NL

\section{- Presentations}

Poster: Annual meeting "Experimental Plant Sciences", Lunteren, The Netherlands

Poster: Annual meeting NWO-ALW Lunteren, The Netherlands Talk: "Thermomorphogenesis" conference, Halle,Germany,

"Phytochrome interactions in plants"

Poster: Annual meeting "Experimental Plant Sciences", Lunteren, The Netherlands

Poster: IGC Symposium -2017 Plant RNA Biology, Oeiras,

Portugal

Talk: Annual meeting "Experimental Plant Sciences", Lunteren,

The Netherlands "Multitalented MED25 mediates transcription"

Talk: "Thermomorphogenesis" conference, Utrecht, NL,

"Feedback regulation of PIF4"

Talk: 7th Dutch Seed Symposium, Wageningen, The

Netherlands, "Phytochromes in seeds"

- IAB interview

- Excursions

Plant Physiology PhD trip, The Netherlands, Germany and

Switzerland

Visit to Dümmen Orange

Visit to Koppert Biological Systems

31 Mar 2016

0.1

29 Apr 2016

0.1

12 May 2016

0.1

13 May 2016

0.1

12 Feb 2017

0.1

$11 \mathrm{Jul} 2017$

0.1

05 Jun 2018

25 Jun 2018

0.1

2-okt-18

25-27 Aug 2016

28-30 Aug 2017

0.9

27-28 Sep 2017

0.6

03-06 Jul 2018

1.2

27-29 Aug 2018

13-14 Apr 2015

1.0

11-12 Apr 2016

1.0

25-27 Aug 2016

1.0

10-11 Apr 2017

1.0

27-28 Sep 2017

09-10 Apr 2018

1.0

27-29 Aug 2018

1.0

02 Oct 2018

22 Apr-01 May 2015

Subtotal Scientific Exposure

\section{3) In-Depth Studies}

- EPS courses or other PhD courses

Postgraduate course: "Basic Statistics", Wageningen, The

Netherlands

PhD course "Bioinformatics-A User's Approach", Wageningen, The Netherlands

\begin{tabular}{c|c}
$\underline{\text { date }}$ & $\underline{c p}$ \\
20-27 May 2015 & 1.5 \\
24-28 Aug 2015 & 1.5
\end{tabular}


Workshop: Root-shoot signalling, Wageningen, The Netherlands

- Journal club

Literature discussions in Plant Physiology, Wageningen, The Netherlands

- Individual research training

2014-2018

Subtotal In-Depth Studies

4) Personal Development

- Skill training courses

EPS Introduction Course, Wageningen, The Netherlands

Efficient writing strategies, Wageningen, The Netherlands

PhD Workshop: Carousel, Wageningen, The Netherlands

Brain Training, Wageningen, The Netherlands

- Organisation of PhD students day, course or conference Co-organization of Erasmus Mundus TIMUR General Assamble, Wageningen, NL

Membership of Board, Committee or PhD council

Herewith the Graduate School declares that the $\mathrm{PhD}$ candidate has complied with the educational requirements set by the Educational Committee of EPS with a minimum total of 30 ECTS credits. 

This work was performed at the Laboratory of Plant Physiology, Wageningen University and financially supported by Erasmus Mundus Action 2 projects TIMUR. The research is funded by the STW Project (13149) "Compact Plants"

Financial support from Wageningen University for printing this thesis is greatly acknowledged.

Cover design and thesis layout by Umidjon Shapulatov

Cover photo: Luciferase activity in transgenic A.thaliana plant

Printed by: www.proefschriftmaken.nl 